Understanding Microstructural

Evolution in Mixed Metal-Oxide

Silsesquioxane Glasses Through

Wet-Chemical Synthesis

Rogier Besselink 


\section{UNDERSTANDING MICROSTRUCTURAL EVOLUTION OF MIXED METAL OXIDE SILSESQUIOXANE GLASSES THROUGH WET-CHEMICAL SYNTHESIS}

Rogier Besselink 


\section{PhD committee}

Chairman and secretary:

Prof. dr. ir. J.W.M. Hilgenkamp University of Twente

Supervisor:

Prof. dr. ir. J.E. ten Elshof University of Twente

Members:

Prof. dr. L. van Wüllen University of Ausburg

Prof. dr. A. Nijmeijer University of Twente

Prof. dr. ing. D.H.A Blank University of Twente

Prof. dr. A.J.A. Winnubst University of Science and Technology

of China, Hefei / University of Twente

Referent:

Dr. A. Petukhov

Utrecht University

Cover: Simulated 2D-small angle X-ray pattern of a mixture of correlated spherical agglomerates with branched polymeric agglomerates. The molecule on the backside represents: $\mathrm{Nb}_{8} \mathrm{O}_{10}(\mathrm{OEt})_{20}$.

The work in this thesis was carried out at the Inorganic Materials Science Group at the faculty of Science and Technology and the MESA+ Institute for Nanotechnology, University of Twente, P.O. Box 217, 7500 AE, Enschede.

This research was financially supported by (STW) the National Institute of Technical Sciences.

R. Besselink: Understanding microstructural evolution of mixed metal oxide silsesquioxane glasses through wet-chemical synthesis.

PhD Thesis, University of Twente, Enschede, The Netherlands

ISBN:

978-90-365-3637-0

DOI:

10.3990/1.9789036536370

Printed by: $\quad$ Gildeprint drukkerijen, Enschede, The Netherlands

Copyright (C2014 by: R. Besselink 


\section{UNDERSTANDING MICROSTRUCTURAL EVOLUTION OF MIXED METAL OXIDE SILSESQUIOXANE GLASSES THROUGH WET-CHEMICAL SYNTHESIS}

\section{PROEFSCHRIFT}

ter verkrijging van

de graad van doctor aan de Universiteit Twente, op gezag van de rector magnificus,

Prof. dr. H. Brinksma, volgens besluit van het College voor Promoties

in het openbaar te verdedigen

op vrijdag 28 maart 2014 om 12:45 uur

door

Rogier Besselink

geboren op 28 februari 1983

te Doetinchem 
Dit proefschrift is goedgekeurd door de promotor

Prof. dr. J.E. ten Elshof 


\section{Table of Contents}

Chapter 1. Introduction 9

1.1) Sol-Gel Chemistry 10

1.2) Sol-Gel Processing 22

1.3) Porous Inorganic Membranes 26

1.4) The Scope of the Thesis and Outline 30

1.5) References 32

Chapter 2. Theory of Small Angle Scattering 39

2.1) Form Function 41

2.2) Structure function 43

2.3) Polydisperse Systems 51

2.4) Non-particulate systems 52

2.5) References 53

Chapter 3. Rapid early stage Schultz-Zimm distributed 57 mass-fractalic growth studies on niobia/silsesquioxane mixtures

3.1) Abstract 58

3.2) Introduction 58

3.3) Experimental section

3.4) Theoretical Background 63

3.5) Results and Discussion 72

3.6) Conclusions 78

$\begin{array}{lll}3.7) & \text { References }\end{array}$ 
Chapter 4. Evolution of microstructure in mixed niobiahybrid silica thin films from sol-gel precursors

Abstract 84

Introduction 84

Experimental Section 86

Derivation of $I(q)$ 88

Results and Discussion 96

Conclusions

References

Chapter 5. Extent of niobia/silsesquioxane intermixing by solid state MAS-NMR

Abstract 
Chapter 6. Crystallized $\mathrm{Nb}_{8} \mathrm{O}_{10}(\mathrm{OEt})_{20}$ from

condensation of niobium ethoxide and acetone that exhibits remarkable anisotropy in thermal expansion

Abstract 144 Introduction

Experimental Section

Results and Discussion

Chapter 7. A novel malonamide bridged silsesquioxane precursor for enhanced dispersion of transition and lanthanide metal ions in hybrid silica membranes 
Chapter 8. Conclusions and Outlook 207

8.1) General Conclusions 208

8.2) Additional observations and remarks 209

8.3) Future Perspective and outlook 210

8.4) References 212

Summary 213

Samenvatting $\quad 217$

Acknowledgement 221

Dankwoord $\quad 225$ 


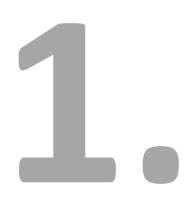

Introduction 


\subsection{Sol-Gel chemistry}

\subsubsection{The chemistry of silicon alkoxides}

Amorphous silicon oxides can be prepared via polymerisation reactions of silicon alkoxides with water. The silicon alkoxides initiate via a hydrolysis reaction (Scheme 1.1) and subsequently propagate via a number of different reaction processes (Scheme 1.2) [1-3]. Propagation mainly occurs via condensation reactions that either involves the elimination of water (oxolation) or the elimination of alcohol (alkoxolation).

$(\mathrm{RO})_{3} \mathrm{Si}-\mathrm{OR}+\mathrm{H}_{2} \mathrm{O} \rightleftharpoons(\mathrm{RO})_{3} \mathrm{Si}-\mathrm{OH} \quad \mathrm{ROH}$

Scheme 1.1. Hydrolysis of silicon alkoxides

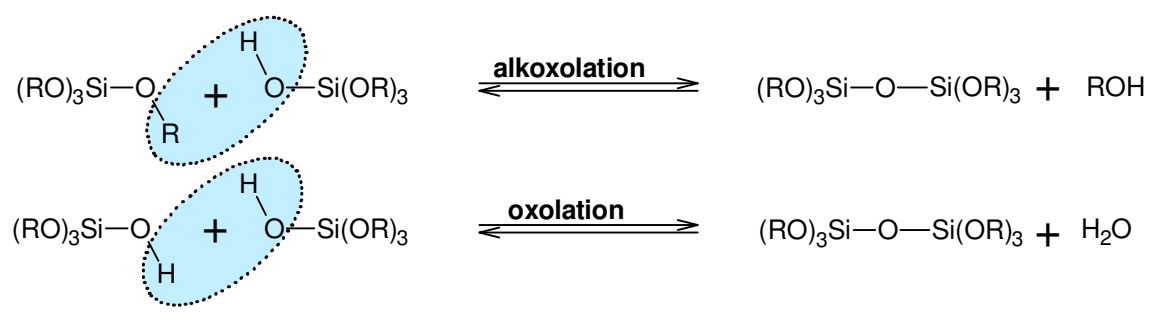

Scheme 1.2. Condensation of silicon alkoxides

In polar solvents, the polymerisation reactions pursue an equilibrium that strongly depends on the reaction conditions. Different structures and morphologies were obtained by tuning suitable reaction conditions, which makes these sol-gel techniques very attractive for a wide range of materials.

Propagation is in general terminated by limited mobility of reactive species that are entrapped into network and by reduction of reactive monomer species. The final product often contains a certain content of alkoxide groups can be removed by calcination. Silicon alkoxides are hydrophobic compounds with a low polarity. This is partly caused by the higher electronegativity of silicon $(1,74)$ in comparison to early transition metal ions in metal alkoxides. A low polarity of silicon alkoxides corresponds with a very low solubility of silicon alkoxides in water [3]. In addition negative partial charge on oxygen of the alkoxide group is further stabilized by a 
mesomeric interaction between occupied $2 p$-orbitals of oxygen and empty $3 \mathrm{~d}$-orbitals of silicon as illustrated in Scheme 1.3 [4]. In contrast to other metal alkoxides, silicon alkoxides are very weak Lewis acids/bases, which corresponds with a hydrolysis rate in water that is orders of magnitudes smaller in comparison to most metal alkoxides [1, 2].
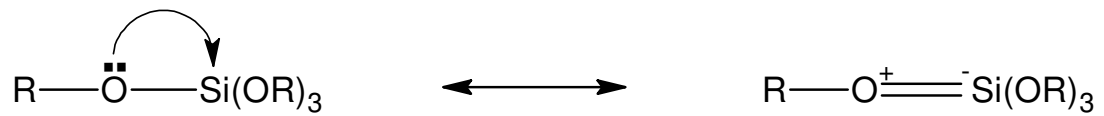

Scheme 1.3. Overlap of between $03 p$ and empty Si 3d orbitals

The $\mathrm{pH}$ has a strong effect on the hydrolysis of silicon, while it determines the stability and the type of transition state and consequently it strongly affect the activation energy of hydrolysis. At a $\mathrm{pH}$ below the isoelectric point (IEP $=2.2$ ) hydrolysis occurs via an acid catalysed mechanism and above IEP via a base catalysed mechanism [1].

The polarity of the silicon oxide bond is partly determined by the type of alkoxide. This can be explained by an inductive effect. The longer the alkane chain of the alkoxide, the stronger is the electron propulsion of this chain which results in a stabilisation of the negative charge of oxygen. Therefore the rate of hydrolysis decreases in the sequence: $\mathrm{Si}\left(\mathrm{OC}_{2} \mathrm{H}_{5}\right)_{4}>\mathrm{Si}\left(\mathrm{OC}_{4} \mathrm{H}_{9}\right)_{4}>$ $\mathrm{Si}\left(\mathrm{OC}_{9} \mathrm{H}_{19}\right)_{4}$ [2]. Steric hindrance can also play a role in the reactivity of alkoxide and the hindrance will increase with increase of the alkyl chain length. Thus, the observed reduction of hydrolysis rate as a function of alkyl chain length is also explained in terms of steric hindrance.

The alkoxide ligands of silicon alkoxide can exchange by a reaction with different alcohols. However, due to the low polarity of the silicon alkoxide bond, dissociation rarely occurs at room temperature and the exchange process takes a number of days. For example: It takes 5 days before $26 \%$ of $\mathrm{Si}(\mathrm{OMe})_{4}$ have exchanged a single methoxide ligand for an ethoxide ligand in ethanol (OMe:EtOH = 1:1) [5]. The dissociation of alkoxide ligands depends on the charge on the partial charge of the silicon atom. In the presence of either Bronsted or Lewis acids the oxigen atoms become more positively charged and therefore the strength of the silicon oxide bond decreases. For acid catalysed systems $90 \%$ of the silicon methoxide precursors are partly substituted within 2 hours [5]. A similar increase was 
observed for Lewis catalysed hydrolysis of silicon methoxide, with either titanium(IV) isopropoxide or vanadium (V) oxy tri-isopropoxide.

\subsection{1.a. Hydrolysis of silicon alkoxides:}

The basic catalysed mechanism occurs via a nucleophilic substitution according to an $\mathrm{S}_{\mathrm{N}} 2$-mechanism, which involves only one intermediate state (Scheme 1.4). The reaction involves the formation of a penta-coordinated negatively charged intermediate. The stability of this intermediate is dependent on the inductive effect of the substituents that surround the silicon atom. The stronger the bacidity of this substituent, the weaker becomes the stability of the negatively charged intermediate. Therefore, the activation energy for hydrolysis decreases with the extent of hydroxylsubstitution in the sequence: $\mathrm{Si}(\mathrm{OR})_{4}<\mathrm{Si}(\mathrm{OR})_{3} \mathrm{OH}<\mathrm{Si}(\mathrm{OR})_{2}(\mathrm{OH})_{2}<$ $\mathrm{Si}(\mathrm{OR})(\mathrm{OH})_{3}$. Hydrolysis is energetically favourable for already hydrolysed species that involves preferably the formation of $\mathrm{Si}(\mathrm{OR})(\mathrm{OH})_{3}$ and $\mathrm{Si}(\mathrm{OH})_{4}$. Condensation of these high functionality hydrolysed species yield typically a highly branched dense structure.
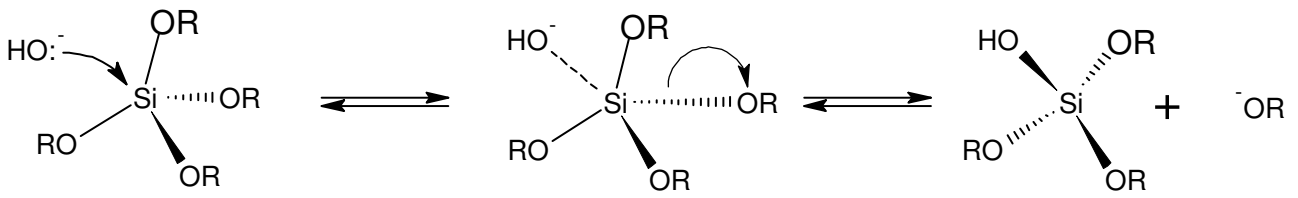

Scheme 1.4. Base catalysed hydrolysis of silicon alkoxides.

In the past, the acid catalysed hydrolysis is often referred to occur via an $\mathrm{S}_{\mathrm{N}} 2$-mechanism, analogous to the basic catalysed mechanism. However, hydrolysis is facilitated when the coordination number is increased from 4 to 5 , by substitution of alkoxide-ligands with glycols [6-8]. In this case a $\mathrm{S}_{\mathrm{N}} 2$ mechanism is unlikely while the additional ligand will decrease the charge of the silicon atom causing the acidity of silicon to decrease. Meanwhile, the silicon oxygen bond length increases to some extent $[6,9]$ and the bond strength has become weaker. Hydrolysis is therefore not initiated by nucleophilic attack on silicon, however most likely via proton transfer towards a basic alkoxide-group. This is consistent a proton assisted $\mathrm{S}_{\mathrm{N}} 1$ mechanism (Scheme 1.5) $[3,10]$. 


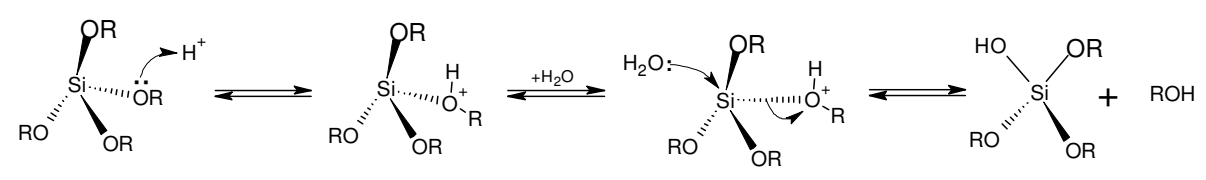

Scheme 1.5. Acid catalysed hydrolysis via a proton assisted $S_{N} 1$ mechanism.

In the first step a positive charge intermediate is formed by an electrophilic attack of a proton on a basic alkoxide group (the rate limiting step). In the second step, an alcohol is substituted by a nucleophilic attack of aqueous oxygen. The stability of the positively charged intermediates decreases with the extent of hydroxyl-substitution in the sequence: $\mathrm{Si}(\mathrm{OR})_{4}>\mathrm{Si}(\mathrm{OR})_{3} \mathrm{OH}>$ $\mathrm{Si}(\mathrm{OR})_{2}(\mathrm{OH})_{2}>\mathrm{Si}(\mathrm{OR})(\mathrm{OH})_{3}$. In contrast to the basic catalysed mechanism the system preferably forms low hydroxyl substituted compounds. Condensation of these compounds yield a weakly branched polymers that still contains a number of alkoxide groups. Gelation of these weakly branched polymers results in an open network structure that can be used for microporous membranes.

\subsection{1.b. Condensation of silicon alkoxides.}

In analogy with the hydrolysis reactions, condensation of silicon alkoxides occurred either through acid or base catalysed mechanisms. The acid catalysed condensation is activated via a proton assisted nucleophilic substitution reaction. The reaction involves a fast protonation step a forms a positively charged intermediate. The second step is slow nucleophilic substitution of an hydroxyl group towards a protonated siloxan molecule (Scheme 1.6)

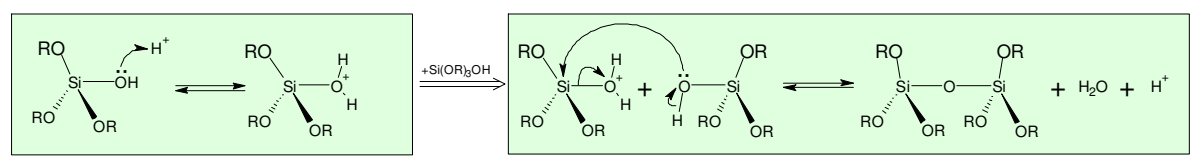

Scheme 1.6. Acid catalysed condensation of silicon alkoxides

The kinetics of acid catalysed hydrolysis and condensation of tetramethyl orthosiloxane (TMOS) and tetraethyl othosiloxane (TEOS) at room temperature was investigated by proton and ${ }^{29} \mathrm{Si}-\mathrm{NMR}$ [11-14]. The hydrolysis rates of TMOS were 30 times larger than the condensation rates, which indicates that condensation, is the rate limiting process. Condensation occurs via both alkoxation and oxolation simultaneously. For 
small hydrolysis ratio's $\left(\mathrm{H}_{2} \mathrm{O}: \mathrm{TMOS}<0.5\right)$ condensation occurs mainly by alkoxation, while oxolation dominates for large hydrolysis ratio's. The speed of protonation of alkoxide and hydroxide groups cannot be determined, while the proton exchange is much faster than the NMR-resonance decay. The measured chemical shift is simply the average of protonated and nonprotonated species.

Increase of the hydrolysis ratio involves an increase of the hydrolysis rate. The increase silanol (SiOH) concentration causes an increase of condensation rate. ${ }^{29} \mathrm{Si}-\mathrm{NMR}$ measurements reveal that sol with an hydrolysis ratio of 4 (H2O:TEOS) contains oligomers with a average size of 3 TEOS units, while in the sol with an hydrolysis ratio of 10 has a average size of 20 TEOS units after 5 hours at room temperature. Nevertheless, the gelation time is 60 days in the case of an hydrolysis ratio of 10 and 35 days in the case with an hydrolysis ratio of 35 . On the other hand, the gelation time of TEOS was increased from 35 days to 60 days for an increase of hydrolysis ratio from 4 to 10 . This clearly suggests that particle growth and gelation is not only determined by the chemical reactions and other aspects including colloidal stability play a crucial role as well.

\subsection{1.c. Silsesquioxanes}

Silsesquioxanes are a particular class of silanes that is described by the formula: $\mathrm{RSiO}_{3 / 2}$, where $\mathrm{R}$ is a carbon containing substituent $[15,16]$. In analogy with conventional silicon alkoxides silsesquioxanes glasses can be synthesized through similar acid or based catalysed hydrolysis and condensation mechanisms. Nevertheless, the versatile chemistry of silsesquioxanes that is compatible organic, polymer and supramolecular chemistry allows a much larger variety of materials to be synthesized. Silsesquioxane precursors can be divided in two different classes: (1) (functionalized) polyhedral oligomeric silsesquioxanes (POSS) and (2) silsesquioxane alkoxides.

POSS molecules contained condensed Si-O-Si cages and the functional rest groups allows these silsesquioxanes to be incorporated in a variety of polymeric structures [17-19]. Recently, self-supportive polyPOSS-imide membrane films were synthesized that unlike conventional polymeric membranes do not suffer from chain-rearrangement-induced permeability loss at elevated temperatures [19] and unlike ceramic membranes these nanocomposits exhibit sufficient strength to remain self-supportive. 
Alternatively, hybrid silsesquioxane glasses can be synthesized through hydrolysis and condensation either pendant or bridged silsesquioxane precursors [16]. A variety of different rest groups can be attached including alkyls, aryls, metalloscenes, crown ethers and cyclames. This allows a tuneable extent of self-organization. For instance, bis-(alkyl-urea)-alkyl bridged silsesquioxanes arrange in a lamellar fashion due to cooperative attractive Van-der-Waals forces between alkyl chains and hydrogen bonds between the urea groups [20]. On the other hand, condensed tetrahedral silsesquioxanes showed only short-range order with very distinct nearest neighbour distances and hardly any long-range anisotropy [21].

Silsesquioxane alkoxides with relatively short bridging groups condense to amorphous glasses, in analogy with conventional silicon alkoxides [22]. Nevertheless, the introduction of hydrophobic alkyl chains within the glassy structure strongly reduced its susceptibility towards hydrolysis [23]. This made this material suitable for pervaporation of waste water under harsh conditions. Moreover, in comparison to conventional glasses the bridged silsesquioxane glasses exhibit substantially higher fracture energies [24, 25].

\subsubsection{The chemistry of metal alkoxides}

Hydrolytic polymerisation of metal alkoxides occurs via hydrolysis and condensation mechanisms that are similar to silicon alkoxides $[3,10]$. However, the more ionic metal alkoxide bond is, the more sensitive it becomes towards hydrolysis and condensation. Transition metal alkoxides readily form metal oxide precipitates in contact with air that contains small fractions of moisture. FTIR-studies of Harris et al. [26] reveal the strong reactivity of titanium ethoxide and zirconium butoxide in water. Acid catalysed hydrolysis occurs readily: within $80 \mathrm{~ms} 36 \%$ and $60 \%$ of the alkoxide groups were hydrolysed of respectively the titanium ethoxide and zirconium butoxide. The measured water concentration maintained constant after $80 \mathrm{~ms}$ indicating that a steady state between hydrolysis and condensation was readily achieved after $80 \mathrm{~ms}$.

The polarity of metal alkoxide bond is higher in comparison to silicon alkoxide bonds, which is partly due to the high partial charge of the metal atom. The partial charge on the metal atom is related with the average difference in Paulings electronegativity between the metal atoms and the 
alkoxide groups [2]. This explains the enhanced reactivity of transition metal alkoxides. Nevertheless, it does not explain why a higher reactivity to hydrolysis/condensation when tin(VI)butoxide is added to silicon ethoxide [27], were the partial charge on tin is smaller $(\delta(M)=0.21)$ in comparison to silicon $(\delta(M)=0.32)$. However the partial charge model [11] does not encounter the resonance effect between empty d-orbitals of silicon and occupied p-orbitals of oxygen as discussed in paragraph 1.1.1. The actual charge of the silicon atom is reduced by p-electrons of oxygen located in $\pi$ orbitals formed by overlap of $p$ and $d$-orbitals. This $\pi$-interaction is less pronounced for heavier elements of group 14 (germanium and tin) in comparison to silicon [7]. Consequently, the real charge of the silicon atom is probably much lower as derived from the partial charge model.

Furthermore, the chance of nucleophilic substitution is possibly enhanced by the chance to increase the coordination number of the metal or metalloid atom. In the case of transition metals, the coordination number is determined by the ionic radius and ligand field stabilisation energy. The situation is more complex in the case of p-block elements, while it involves more covalent metal/metalloid oxide bonds. The coordination number of silicon can be increased from four to five or six, which involves a hybridisation with empty $d$-orbitals. The existence of five and six coordinated silicon alkoxide complexes are reported in literature [6]. However, (spd)-hybridization is competitive to resonance effect between oxygen and a metal/metalloid centre. In the particular case of silicon the driving force to increase coordination number is strongly reduced by the extent of this resonance effect. Therefore, depending on the type of ligand, silicon complex have typically fourfold coordinated, while most germanium or tin complexes have a six-fold coordination.

On the other hand, the nucleophilic substitution mechanism of metal alkoxides does not necessarily involve expansion of the coordination number. Metal alkoxides tend to increase their coordination number via oligomerization. The saturated coordination numbers have already been achieved for a large range of metal alkoxide precursors and coordination expansion is not necessary the main driving force in hydrolysis. Transition metals of period 4 tend to have a coordination number of 6 . For instance solid crystalline titanium (IV) ethoxide has a tetrameric structure (Figure 1.1), where titanium has a sixfold coordination of ethoxide ligands [28]. The tetramer contains two alkoxide ligands that are coordinated to three 
titanium ions ( $\mu_{3}$-bridged ligands) and four alkoxy ligand that are coordinated to two tinanium ions ( $\mu_{2}$-bridged ligands). The chance of nucleophilic addition has reduced for a tetramer in comparison to the monomer, while titanium has a higher coordination number in the tetramer. On the other hand, partial charge calculations [29] reveal that $\mu$ bridged ligands have a higher partial charge in comparison to pendant ligands. For the titanium metoxide tetramer, the oxygen partial charges are respectively -0.74 for $\mu_{3}-0,-0.66$ for $\mu_{2}-0$ and the partial charge for pendant ligands lies in range between -0.48 and -0.58 . The bridged ligands are therefore stronger bases that are more susceptible towards protonation and will be hydrolysed at first. The formation of tetramers is not possible for titanium alkoxides that have large bulky alkyl chains, for instance crystalline titanium (IV) neopentoxide is dimeric [30] and crystalline titanium (IV) 2,6-isopropyl phenoxide is monomeric [31].

An alcoholic solution of alkoxide precursors does not always consist of one particular type molecule and often, it is a mixture of monomers and different types of oligomers that are in equilibrium with each other. The exact composition of a metal alkoxides dissolved in a alcohol is not always known and the chemistry of these alkoxides, even without the addition of modifiers, can be quite complex.

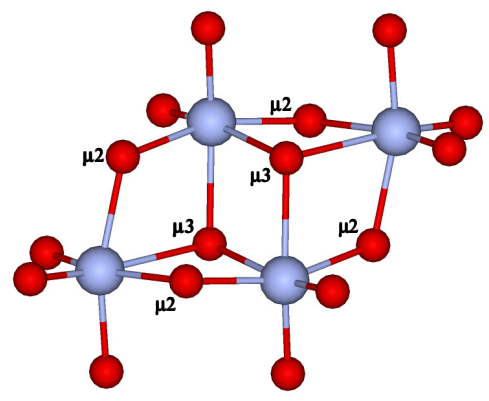

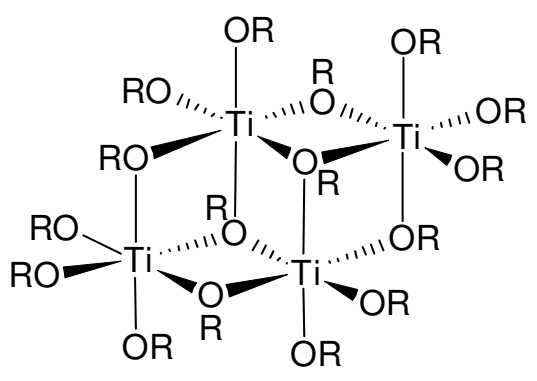

Figure 1.1. Oligomeric arrangement of a tetrameric titanium ethoxide.

Among all metal alkoxides, titanium and zirconium alkoxides have received most attention in the past decades. Nevertheless, the disadvantage of these elements in the preparation of membranes is their tendency towards the 
formation of dense crystalline particles. (examples titanium \& zirconium crystallisation). Moreover, the heterometalic bonds in mixed titanium silsesquioxanes or zirconium silsesquioxanes derived products (ie.: Ti-O-Si and $\mathrm{Zr}$-O-Si-bonds) rearrange in homonuclear bonds (ie. $\mathrm{Ti}-\mathrm{O}-\mathrm{Ti}, \mathrm{Zr}-\mathrm{O}-\mathrm{Zr}$ and $\mathrm{Si}-\mathrm{O}-\mathrm{Si})$ due to hydrolytic cleavage $[32,33]$. Both the presence unreacted hydroxyl groups or the presence water enables this rearrangement towards thermodynamically more stable homonuclear bonds.

Amorphous oxides that were prepared from niobium penta-ethoxide gels were found to remain amorphous up to $400^{\circ} \mathrm{C}$ [34]. Based on DSCmeasurements, the majority of niobium pentoxide crystallized at $578^{\circ} \mathrm{C}$ : A crystallisation temperature that is much higher than the crystallisation temperature of either zirconium or titanium oxide. Thus, in the case of niobium oxides the tendency to form separate crystalline clusters would be smaller. The Niobium penta-ethoxide molecule is a dimer that consist of two niobium atoms in a distorted octahedral of oxygen atoms [34]. Each niobium atom is surrounded by four pendant ethoxide groups and two ethoxide groups that are connected with the other niobium atom via $\mu$ bridges. The polymerization niobium pentoxide precursors yields the formation of complex polymeric clusters of distorted corner-sharing and edge-sharing octahedral[34].

\subsubsection{Modifiers for alkoxide polymerization.}

Doping of metal alkoxide in a silica-matrix is still difficult due to the high reactivity of the metal alkoxides. Homocondensation of metal alkoxides is preferred over heterocondensation with silicon alkoxides even when modifiers were added to reduce the reactivity of metal alkoxides. The calcined oxide will contain metal rich phases of metal oxide phases in a silica matrix that were observed by SAXS and TEM measurements for calcined mixed zirconium silicon xerogels [35]. Furthermore, the tendency of metal alkoxides to increase the coordination number results in the formation of dense crystalline domains and strongly reduce the microporosity of the amorphous silica matrix eg: Calcination of titanium doped silica xerogels yields the formation of anastase and rutile domains of titanium dioxide. The extent of phase separation and densification can be reduced with the addition of inhibitors or modifiers. These modifiers may affect the relativities of metal alkoxides in different ways as discussed below. 
1. Inductive effect: The inductive effect can be explained in terms of partial charge model of Livage et al. [2]. The partical charge on the metal cation is stabilized by electron propulsion of the alkyl chain that increases with both the length and the extent of branching (Scheme 1.7).

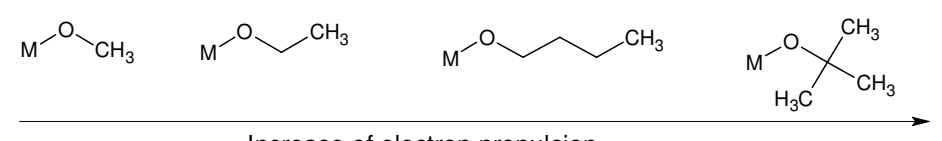

Increase of electron propulsion

\section{Scheme 1.7. Inductive effect of ligands}

2. Mesomeric effect: Titanium and tungsten phenoxides are less susceptible for hydrolysis in comparison to alkoxide equivalents [2,36]. Phenoxides are known as a strong $\pi$-donor ligands that stabilizes the positive partial charge of the metal cation. Surprisingly silicon phenoxide was found to be more reactive towards hydrolysis. Probably, the mesomeric effect plays a minor role in the case of silicon and the increase of reactivity was possibly caused by the inductive effect of the phenoxide (Scheme 1.8).

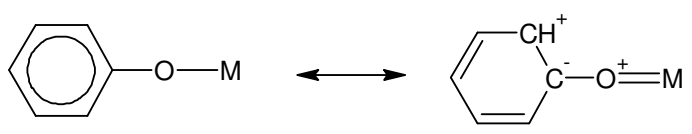

Scheme 1.8. Mesomeric effect of phenoxides

3. Chelating effect: Substitution of a number of monodentate ligands for a multidentate ligand is entropically favourable while the number of reaction products is higher than the number of reactants [37]. Furthermore, bond formation with multidentate ligands is often cooperative in a positive sense and therefore also favourable from an enthalpic point of view. Calorimetric measurements of Blanchard et al. [37] reveal an increase of enthalpy when alkoxide ligands of zirconium or titanium were substituted by bidentate acetylacetonate ligands. Titanium(IV)-alkoxides were found to react with acetylacetone up to a metal/chelating ligand-ratio of two $(r=2)$. Further addition of acetylacetone did not involve heat formation and the enthalpy remains constant. The existence of dimeric $\left[\mathrm{Ti}(\mathrm{OR})_{3} \mathrm{acac}\right]_{2}$ with $\mathrm{r}=1$ and monomeric $\mathrm{Ti}(\mathrm{OR})_{2} \mathrm{acac}_{2}$ with $\mathrm{r}=2$ where confirmed by $\mathrm{X}$-ray crystallography [38]. In both cases the titanium ion is sixfold coordinated, which is the optimal coordination state of titanium. In the particular case of zirconium npropoxide acac-substitution occurs up to a complexation ratio of $r=4$, which 
involves the formation of a stable $\mathrm{Zr}(\mathrm{acac})_{4}$ molecule [39]. Zirconium has a larger ionic radius in comparison with titanium and therefore it can increase their coordination number to 8 in the case of $\mathrm{Zr}(\mathrm{acac})_{4}$. The $\mathrm{Zr}(\mathrm{acac})_{4}$ molecule is very stable towards hydrolysis even at an excessive amount of water and therefore not suitable for microporous materials. Spijksma [39] has shown that for smaller complexation ratio's partly substituted zirconium propoxides were formed (for both iso and n-propoxide): sixfold coordinated $\left[\mathrm{Zr}(\mathrm{OPr})_{3} \mathrm{acac}\right]_{2}$ and sevenfold coordinated $\left[\mathrm{Zr}(\mathrm{OPr})(\mathrm{acac})_{3}\right]$. However, during aging these compounds slowly rearrange in both $\mathrm{Zr}(\mathrm{OPr})_{4}$ and $\operatorname{Zr}(\mathrm{acac})_{4}$.

The number of functional groups reduces by substitution with acetylacetone and as a result the extent of branching reduces as well. This is in agreement with a reduction of a fractal dimension (for $r>0.5$ ) that was reduced from 2.5 to 1.6 for a sol of yttrium doped zirconium propoxide [40]. Futhermore, the growth rate of the sols were reduced, which agrees with a reduction of the radius of gyration.

4. Bridging effect of ligands: Related to the geometry and flexibility, particular multidentate ligands form bridges between alkoxides. Bridge formation has two advantages: Firstly the intermixing of different type of alkoxides will be improved by bridge formation between different alkoxides. Furthermore, this opens a pathway for the synthesis of single source precursors that contains different types of metal or metalloid alkoxides. Secondly, molecular weight increases with the extent of bridge formation and therefore it reduces the diffusitivity of the molecular building block in solution. At some point bridge formation result in diffusion limited growth that yields a limited and better controlled growth of sol particles. Furthermore, the extent on branching is reduced by diffusion limited growth, yielding a more porous open network structure.

Mixed single source zirconium/titanium and hafnium/titanium precursors were successfully prepared with diethanol amine as a bridging ligand [41]. The isolated crystals contain zirconium (or hafnium) atom and two titanium atoms that were interconnected by three diethanol ligands. The reactivity of the single source precursor is solely determined by the titanium alkoxide groups and the more reactive zirconium centre was completely isolated. Membrane preparation of the precursor yields a homogeneous product that maintains homogeneous up to a calcinations temperature of $650^{\circ} \mathrm{C}$ [42]. The membrane showed a good selectivity for hydrogen/butane 
mixtures, with a permselectivity of 54 . The selectivity between hydrogen / methane mixtures was substantially smaller with respect to conventional silica membranes, which indicates that the mixed metal oxide precursor yields a larger pore structure.

Bridging ligands are useful in the preparation of mixed metal oxide precursors, although silicon alkoxide behave different. Firstly, ligand exchange is a much slower for silicon alkoxides and secondly silicon rarely increases their coordination number to 5 or 6 . As a result the binding affinity of multidentate ligands is larger towards metal alkoxides. A different strategy was applied Puchberger et al $[43,44]$, were single source precursors were prepared via a covalent linkage between silicon and an organic diketonate ligand. The diketonate ligand is a chelating ligand that forms two ionic bridges with titanium isopropoxide.

\subsubsection{Heterogeneous bond formation through nonhydrolytic pathways}

A complete different strategy towards homogeneous mixed metal oxide doped is a more selective condensation between two different functional groups. These reactions occur via a transesterification reaction without the presence of water and were therefore referred as nonhydrolytic condensation mechanism. Trans-esterification can occur through ester elimination or alkyl halide elimination mechanism [45]. The ester elimination mechanism is based on a reaction between a metal or metalloid alkoxide with a metal or metalloid carboxylate according to the following scheme:

$\equiv \mathrm{M}-\mathrm{OR}+\mathrm{R}-(\mathrm{C}=\mathrm{O})-\mathrm{O}-\mathrm{M}^{\prime} \equiv \rightarrow \equiv \mathrm{M}-\mathrm{O}-\mathrm{M}^{\prime} \equiv+\mathrm{R}-(\mathrm{C}=\mathrm{O})-\mathrm{OR}$

Scheme 1.9 Condensation through ester elimination.

The alkylhalide elimination reaction occurs as follows:

$\equiv \mathrm{M}-\mathrm{OR}+\mathrm{X}-\mathrm{M}^{\prime} \equiv \rightarrow \equiv \mathrm{M}-\mathrm{O}-\mathrm{M}^{\prime} \equiv+\mathrm{X}-\mathrm{R}$

Scheme 1.10 Condensation through alkyl halide eleimination.

In the case of transition metals both ester and alkylhalide elimination reaction occurs around $100^{\circ} \mathrm{C}$ [45]. The reactivity of silicon alkoxides is 
enhanced by metal complexes that act as Lewis acids. This strategy was successfully applied between silicon acetate or methylated silicon acetate and several metal alkoxides [27, 29-32, 45] (eg titanium, zirconium, tantalium and tin alkoxides).

The choice of a proper solvent plays in important role in the selectivity to either ester elimination or ligand exchange. When using pyridine in the reaction between silicon acetate and tin(IV) tert-butoxide the reaction was dominated by ligands exchange, while in the case using toluene as a solvent the reaction was dominated by ester elimination [32] Pyridine is a strong coordinating solvent due to the presence of the nitrogen lone pair. Hence, it forms strong electrostatic interactions with the tin moieties which effectively block the free coordination sites of tin and disable the ester elimination process. Toluene, on the other hand is a very apolar solvent and will not coordinate the tin atom.

\subsection{Sol-Gel Processing.}

\subsubsection{Nucleation and Particle growth.}

Sol-Gel processing of alkoxides starts with the formation of solids from a solution of monomeric or oligomeric precursor molecules. Molecules become attached to each other via hydrolysis and condensation reactions (paragraph 1.1) and form oxygen bridged polymeric structures. As the polymer size increases the solubility decreases and above a critical size particles are nucleated. Nucleation of particles involves the formation of solid liquid interfaces and the properties of the interfaces have a strong influence on growth and cluster formation of polymeric species. At this critical moment particles can either precipitate or form a heterogeneous colloidal dispersion of particles: A process that depends on the balance between repulsive and attractive forces between the solid interfaces of particles. 


\subsubsection{Colloidal stability}

Stability of colloids are often described by the DLVO-theory that considers two distinct forces, namely Van-der-Waals (VdW) forces and Coulomb forces: VdW forces are attractive forces, arising from dipole/dipole or induced-dipole-induced-dipole interactions and coulomb forces are repulsive when sol-particles contain the same charge. The metal oxide clusters become charged due to either the adherence of negatively charged hydroxide or alkoxide ions on the metal atoms or the adherence of protons on the oxygen atoms. The extent in which either positively or negatively charged ions adhered to the sol particle surface depends on the nucleophilic / electrophilic character of the sol particle with respect to the $\mathrm{pH}$. At a certain $\mathrm{pH}$, the so-called isoelectric point (IEP) the particle is $\mathrm{pH}$ neutral. Stable sols are formed either below IEP, with the formation of positively charged sol-particles of above IEP with the formation of negatively charged sol-particles. Nevertheless, not all sol particles have the same isoelectric point, for instance due to size differences that causes differences in inductive effects. More importantly, large differences can be observed in the case of heterogeneous precursors with different metal/metalloid nuclei.

The DLVO theory is only applicable in the cases repulsion is mainly of an electric origin [46]. However, even at a high ionic strength [33] agglomeration of colloids did not always occur and rather uniform particles were stabilized. In such cases additional short range repulsive forces could play a predominant role. Short range repulsions were probably caused by a solvation barrier or steric hindrance. Particularly, in the case of metal and metaloid alkoxides that show a weak tendency to dissociate, the charge of nucleated particles could be limited. The strength of both the solvation barier or steric barier is effected by the type of ligands that surround the growing particle. For instance, nanosized titania particles (15 - 40 Angstrom) were stabilized in a sol which contains both acetylacetone and paratoluene sulfonic acid [36]. 


\subsubsection{Gellation process}

Acid catalized polymerization of silion alkoxides results in the formation of weakly branched polymers. The polymer chain length increases in time, which involves an increase of viscosity of the alkoxide mixture. As the concentration of monomer the change of a formation of a linkage between polymer chains will increase. At the Gel-point polymer chains are linked together and formed a three-dimensional flexible network. This involved an change of a viscous polymer solution into a elastic gel-network. This transition is often measured by rheologic measurements as the point where the viscosity rapidly goes to infinity. However, in theory at the equilibrium point of gelation, the viscosity is infinite. The transition point should be determined by extrapolation that induces a large experimental error.

This problem can be avoided by oscillary shear experiments, while the applied strain is limited [40]. An oscillating strain is applied and the measured stress characterizes the visco-elastic behaviour. In the case of an elastic material the measured stress $\sigma(t)$ is proportional to the applied strain $\varepsilon(t)$.The elastic behaviour is therefore characterized by the slope of the stress versus stain curve, the storage modulus $G^{\prime}$. In the case of a viscous liquid the measured stress is delayed in comparison to the applied strain. This occurs because of two phenomena: Firstly, the liquid molecules slip over each other, while in the case of an elastic network the intramolecular connections contracts the material to his original position. Secondly, a floating liquid has a certain momentum and additional strain is required to stop the liquid flow. The maximum stress is measured at the point where the stress is reversed while the viscous sol is still floating. This viscous behaviour is related with the second derive of the stress as a function of stain, the loss modulus $G^{\prime \prime}$.

Then, after the point of gelation, further development of the structure occurs during the aging process. The polymer chains are flexible and bend in order to form linkages between two chains and as a result, a certain amount of stress develops in the gel network. The gel-network contracts with the expulsion of an amount of solvent expulse through the pores. This stage of shrinkage is called: syneresis. Meanwhile, contraction occurs by a reduction of surface energy. Small pores will disappear, while two solid surfaces will approach each other. This involved a reduction of solid-liquid interface, thus a reduction of surface energy. Syneresis is a self limiting 
process, which slows down by decreasing network permeability and increasing stiffness of the gel network.

\subsubsection{Drying Processes}

The drying process of silica-gels is well described by the Capillary mechanism. In the pores the overall pressure is increased by the capillary pressure and therefore the extent of evaporation at certain temperature was reduced. The capillary pressure $P_{\mathrm{c}}$ was determined by the curvature of the meniscus of the solvent-liquid interface. At the surface of gel that contains pores the curvature of the meniscus is determined by the pore radius. Furthermore, with the assumption of cylindrical shaped pores the capillary pressure is described by the Kelvin equation [47]:

$$
P_{c}=\frac{2 \cdot \gamma_{L V} \cdot \cos (\theta)}{r_{c}}
$$

The formation of porous gels requires good wetting of the solid material and the contact angle $\theta$ should be smaller than $90^{\circ}$ and therefore the capillary pressure is always positive in these gels. During evaporation the solvent at the pore endings the curvature of the solvent menicus increases, which correlates with a reduction of the curvature radius $r_{c}$ and therefore causes an increase in capillary pressure. The curvature radius decreases to a critical point at which the curvature radius approaches the pore radius and the capillary pressure will reach its maximum (Figure 1.2). This is the moment at which dry shrinkage stops and at which the pore size is established $[48,49]$. The volumetric strain $(\varepsilon)$ at this point is related with the bulk modulus $K_{\mathrm{p}}$ and the volume fraction of solid material $\left(\phi_{\mathrm{s}}\right)$ by the following equation: 


$$
\varepsilon=\frac{1-\phi_{\mathrm{S}}}{K_{\mathrm{p}}} \cdot P_{\mathrm{C}}
$$
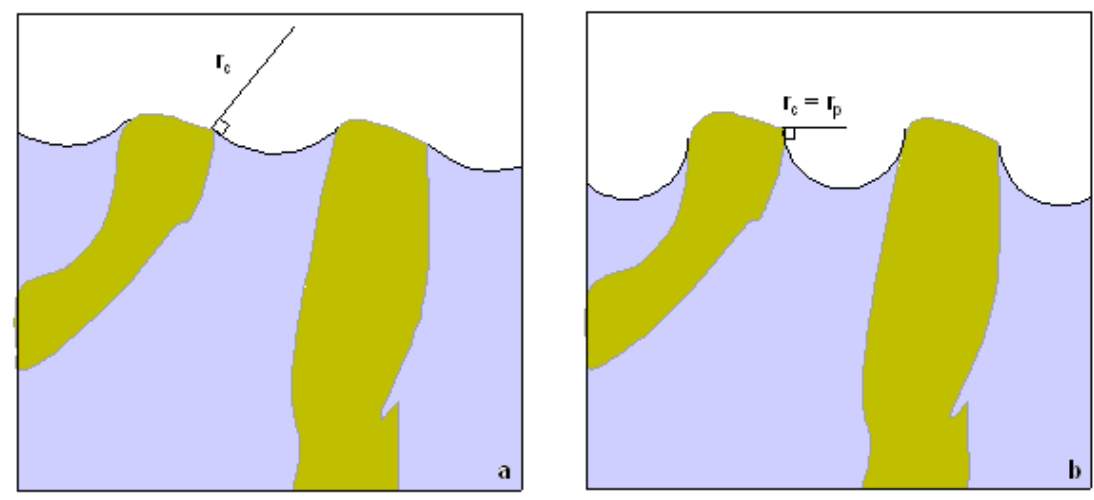

Figure 1.2 Illustration change in curvature of the solvent while drying a porous network structure going from (a) to (b).

\subsection{Porous Inorganic Membranes}

Inorganic membranes are being divided into two classes ion conducting membranes [50] and porous membranes [51-54]. Their transport mechanisms are completely different. Ion conducting membranes are dense and only allow transport of specific ions, whereas porous membranes can be compared with molecular sieves.

Porous membranes are existent in a variety of different pore sizes ranging from macroporous $(>50 \mathrm{~nm})$, mesoporous $(2-50 \mathrm{~nm})$ and microporous membranes $(<2 \mathrm{~nm})$ [54]. Sol-gel derived macro- and mesoporousmaterials can be synthesized as a assemble of colloidal particles, or with the use of a template (a binder or surfactant) that is being burned off $[49,54]$. On the other hand the formation of micropores that are close to the size of individual atoms always relies on the chemistry of atoms that are forming these pores. Therefore, the tuning size of micropores is more difficult and it required a proper understanding of the chemistry that is involved. 
Microporous materials can be divided into two type of materials: (1) crystalline materials (such as metal organic frameworks and Zeolites [53, $55])$, and (2) glassy networks $[47,56]$. Crystalline membranes have a ideal pore size distribution and can yield high fluxes, however these materials cannot reach the small pore sizes of sol-gel derived silica glasses [57]. Consequently, their selectivity for small gas molecules including $\mathrm{CO}_{2}, \mathrm{~N}_{2} \mathrm{O}_{2}$ $\mathrm{CH}_{4}$ and $\mathrm{H}_{2}$ is limited. Moreover, these materials are more susceptible for defects at the grain boundaries. On the order hand silica membranes are more selective, are less susceptible to defects and can be applied to different geometries, such as tubular shaped membranes [54, 56-58].

Despite their high selectivity the applicability of silica membranes was limited due to their limited hydrothermal and mechanical stability [23, 25, 52]. However, with the introduction of bridged silsesquioxanes (1,2-bis(triethoxysilyl)-ethane (BTESE)) membranes were manufactured that remained stable for 2 years at $T=150^{\circ} \mathrm{C}$ while separating water in water butanol mixtures [23]. The improvement of hydrothermal stability was dedicated to (1) the presence of Si-C-C-Si bonds that are not susceptible to hydrolysis and (2) the increased hydrophobicity that repels water from the Lewis acidic Si-nuclei. Consequently, these materials exhibit substantially higher fracture energies (BTESE: $6.95 \mathrm{~J} / \mathrm{m}^{2}$ ) as compared to conventional silica $\left(\mathrm{SiO}_{2}=4 \mathrm{~J} / \mathrm{m}^{2}\right)[24]$.

Neither conventional silica nor bridged silsequioxane membranes are sufficiently strong to be self-supportive. Therefore, a selective layer in a size range in between $50-200 \mathrm{~nm}$ is macro/mesoporous support. A macroporous support of $\alpha$-alumina with a mesoporous support of $\gamma$ alumina is frequently being used for this purpose as illustrated in Figure 1.3 


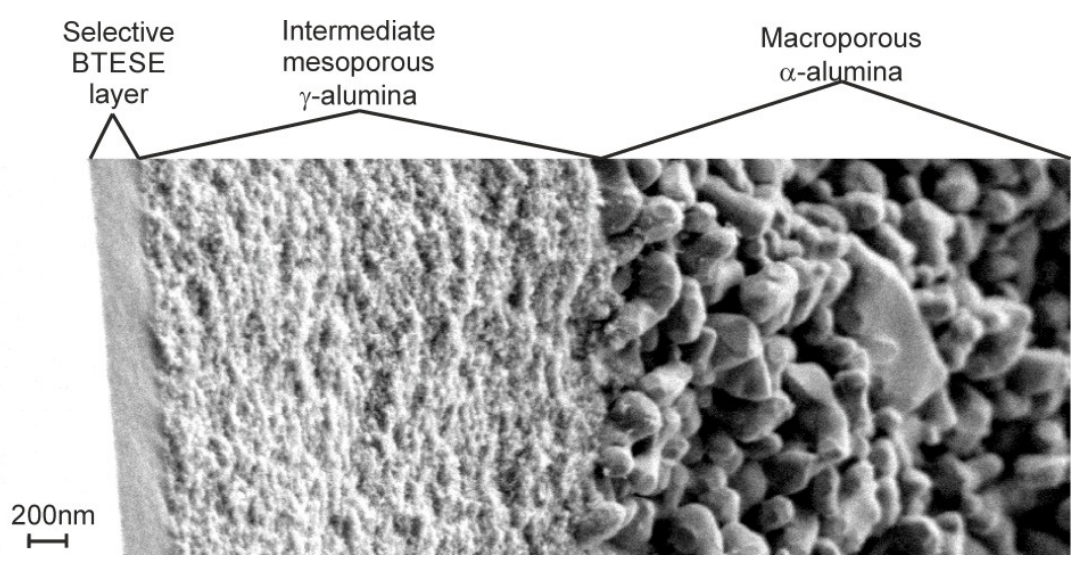

Figure 1.3. Secondary electron scanning microscopy image (SEI-SEM) of a dipcoated BTESE sol onto a meso/maco-porous $\gamma-\mathrm{Al}_{2} \mathrm{O}_{3} / \alpha-\mathrm{Al}_{2} \mathrm{O}_{3}$ support.

\subsubsection{Transport mechanisms in porous membranes}

The permselectivity of a porous membrane strongly depend on the type of transport mechanism that is involved. The transport mechanisms depend on the size difference pores and the molecules being transported different mechanisms can occur as illustrated in Figure 1.4 [54]. If the pores are substantially larger as compared to the molecules transport occurs trough viscous flow. Due to shear tension at the pore surface large velocity differences exhibit between similar sized molecules. Consequently, such systems are poorly selective and strongly pressure dependent.

In the case of Knudsen diffusion, molecules bounces ballistically between pore walls. The flux of such systems is determined by the frequency that molecules bounce against the pore wall. Large molecules experience a smaller free path length as compared to smaller molecules and will bounce more frequently against the pore walls. Consequently, large molecules will experience a large friction as compared to small molecules. 


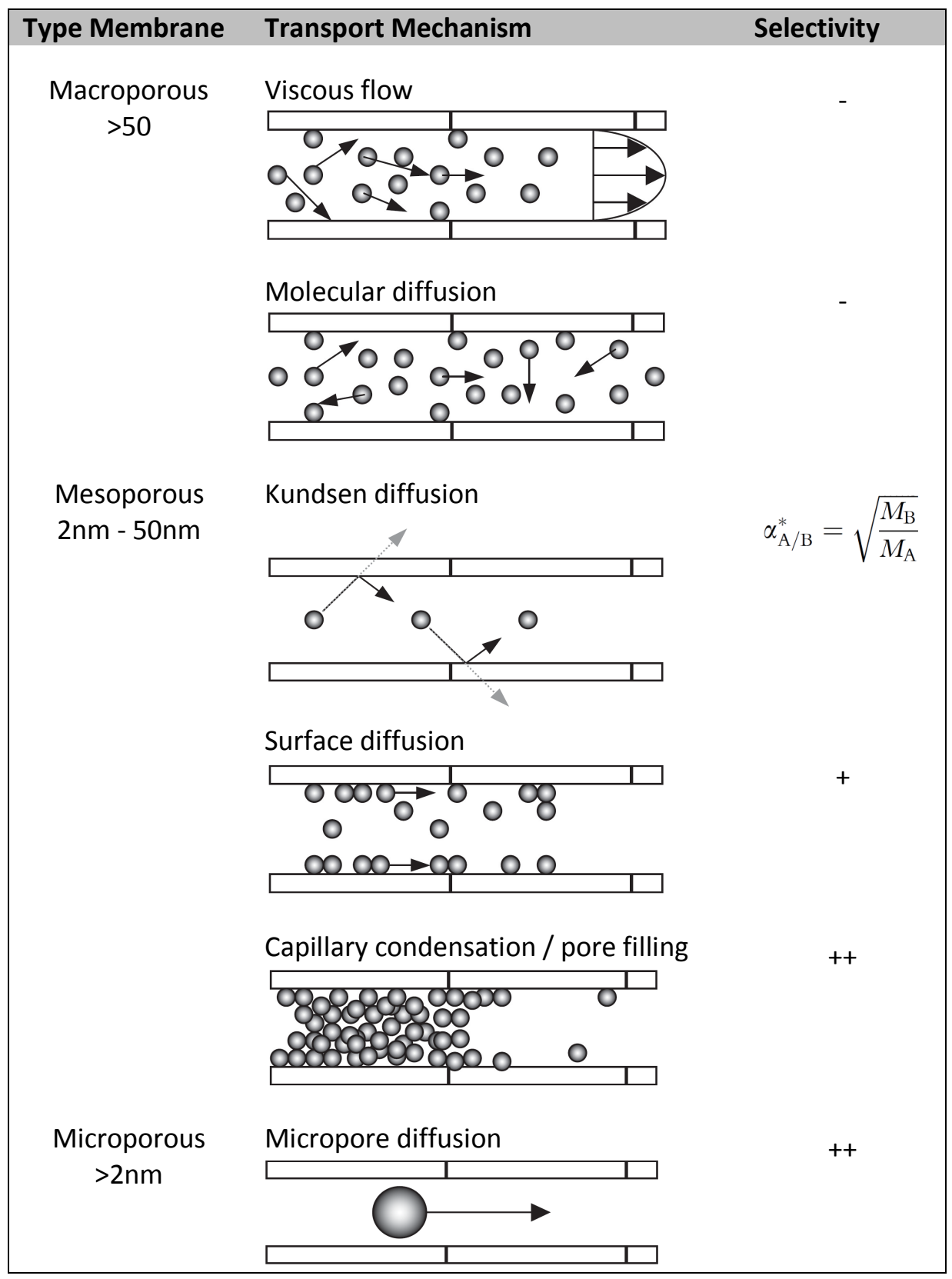

Figure 1.4. Transport mechanism through porous membranes. Reprinted from [54] with permission from Elsevier. 
In the ideal case were pores are only slightly larger than one type of molecule, while other molecules are being expelled. In that case infinitively high selectivities can be reached through microporous diffusion of only one particular type of molecules. Diffusion is referred to as microporous diffusion if size of the pores is sufficiently small such that the molecules that go through the membrane continuously experience $\mathrm{VdW}$ forces of the surrounding membrane materials. In such case no distinction can be made between bulk and surface diffusion. Nevertheless, some molecules such as a mixture of $\mathrm{N}_{2}$ and $\mathrm{CO}_{2}$ have similar kinetic diameters of $3.30 \AA$ and $3.64 \AA$, which are extremely difficult to separate solely by their difference in size [53].

\subsection{Scope of this Thesis and Outline}

The selective membrane layer of condensed silsesquioxanes may be altered by incorporating metal oxides within the matrix. Doping metal oxides was found useful either (1) to manipulate the membranes selectivity for instance by scavenging $\mathrm{CO}_{2}$ with selective sites of $\mathrm{Nb}_{2} \mathrm{O}_{3}$ [59] and (2) to improve its hydrothermal stability [60-62]. Nevertheless, the realization of homogeneously distributed metal centres within a condensed silsesquioxane matrix is not straightforward from a synthesis point of view. Such systems tend to phase separate due to the mismatch in reactivity between the precursors being used. The synthesis is often optimized by trial and error and the evolution of the microstructure of such systems is poorly understood. This thesis is primarily focussed on monitoring and understanding different stages of the sol-gel chemistry of mixed metal alkoxide-silsesquioxane systems, and how these processes affect the final microstructure. It may allow us to find more systematic solutions to the mismatch in reactivity of metal alkoxides versus silicon alkoxides.

One technique that allows a relatively fast monitoring (up to $3 s$ ) of microstructural evolution is Synchrotron Radiation Small Angle X-ray Scattering (SAXS). Nevertheless, the interpretation of such data is not straightforward. The acquired data represent a probability distribution on a reciprocal space axis that is related to the probability of finding distance correlations between or within electron dense domains. Such data cannot be immediately translated into a true electron density map without making certain assumptions. Chapter $\mathbf{2}$ describes the theory of SAXS in short. It 
deals with the (1) the transformation between real and reciprocal space, and (2) the interpretation and applicability of particular models.

In-situ SAXS was used to monitor microstructural changes in mixed niobium pentaethoxide (NPE) / 1,2-bis-(triethoxysilyl)-ethane (BTESE) sol-gel systems as described in Chapter $\mathbf{3}$ and Chapter $\mathbf{4}$. Chapter $\mathbf{3}$ evaluates particle agglomeration processes in the very early stages of sol particle formation just after mixing alkoxide mixtures with acidified water diluted in ethanol. The agglomeration processes presumably occurs through irreversible Smoluchowski type agglomeration kernels that result in massfractalic agglomerates. Experiments in the presence and absence of BTESE revealed a substantial influence of BTESE on the agglomeration process of NPE.

Chapter $\mathbf{4}$ is focused on the microstructural evolution of similar sols during the drying processes. These drying processes are characterized by three stages: (1) an induction stage in which growth of primary building blocks was observed, (2) a transition stage during which the solvent disappeared and a contrast between $\mathrm{Nb}$-rich and Si-rich domains appeared and (3) a final densification stage. The microstructural evolution depends on the moment that niobium pentaethoxide was add to the system while preparing the mixed sols.

The network organization was characterized by solid state ${ }^{13} \mathrm{C},{ }^{29} \mathrm{Si}$ and ${ }^{17} \mathrm{O}$ solid state NMR as described in Chapter 5. Solid state NMR spectroscopy allows the assignment of specific atoms to be attached to the measured nuclei. These techniques provide insight into the extent of remnant organic residues, condensed groups and heteronuclear $\mathrm{Si}-\mathrm{O}-\mathrm{Nb}$ linkages as being assigned by ${ }^{17} \mathrm{O}-\mathrm{NMR}$. The influence of chelating ligands acetylacetone and 2-methoxyethanol was examined. Acetylacetone in particular was found to improve the distribution of niobium within the silsesquioxane mixtures.

The condensation reaction of niobium ethoxide is controlled more easily by replacing water for a less nucleophilic oxolation source such as acetone as described in Chapter 6. Niobium ethoxide condenses slowly through aldol addition and ether elimination mechanisms which consequently crystallized as $\mathrm{Nb}_{8} \mathrm{O}_{10}(\mathrm{OEt})_{20}$. Single $\mathrm{X}$-ray crystallography revealed a remarkable thermal anisotropy of the refined crystals that were measured at $T=150 \mathrm{~K}$ and $T=296 \mathrm{~K}$. 
Alternatively, the dispersion of metals within the silsesqioxane matrix may be improved by a proper design of the silsesquioxane monomer. Fortunately, the chemistry of silsesquioxanes is compatible with organic chemistry. Therefore, it allows the attachment of specific groups being capable of coordinating metal centers. Chapter $\mathbf{7}$ is specifically focused on the synthesis and applicabilition of a malonamide bridged silsesquioxane $N, N, N^{\prime} N^{\prime}$-tetrakis-(triethoxysilylpropyl)-malonamide (referred to as TTPMA). TTPMA enhances the dispersion of $\mathrm{Ce}^{4+}$ and $\mathrm{Ni}^{2+}$ by coordinating these metals before the addition of acidified water. This chapter contains an extensive evaluation of the synthesis and characterization of Ce-TTPMA and $\mathrm{Ni}$-TTPMA coated composite membranes.

\subsection{References.}

1. Brinker, C.J.S., G. W. , Sol Gel Science, The Physics and Chemistry of Sol-Gel Processing. Vol. 1. 1990, San Diego: Acedemic Press. 1-881.

2. Livage, J., M. Henry, and C. Sanchez, Sol-gel chemistry of transition metal oxides. Prog. Solid State Ch., 1988. 18(4): p. 259-341.

3. Kessler, V.G. and G.A. Seisenbaeva, New Insight into Mechanisms of Sol-Gel Process and New Materials and Opportunities for Bioencapsula-tion and Biodelivery, in Sol-Gel Methods for Materials Processing. 2008, Springer. p. 139-153

4. Sanchez, J. and A. McCormick, Kinetic and thermodynamic study of the hydrolysis of silicon alkoxides in acidic alcohol solutions. J. Phys. Chem., 1992. 96(22): p. 8973-8979.

5. Yoshida, H., et al., Control of the structure of niobium oxide species on silica by the equilibrium adsorption method. Catal Today, 1996. 28(1): p. 79-89.

6. Rankin, S.E. and A.V. McCormick, Hydrolysis pseudoequilibrium: challenges and opportunities to sol-gel silicate kinetics. Chem. Eng. Sci., 2000. 55(11): p. 1955-1967.

7. Harris, M.T., et al., FTIR spectroscopy, SAXS and electrical conductivity studies of the hydrolysis and condensation of zirconium and titanium alkoxides. J. Sol-Gel Sci. Technol., 1997. 8(1-3): p. 4147.

8. Simonsen, M.E. and E.G. Søgaard, Sol-gel reactions of titanium alkoxides and water: influence of $\mathrm{pH}$ and alkoxy group on cluster 
formation and properties of the resulting products. J. Sol-Gel Sci. Technol., 2010. 53(3): p. 485-497.

9. Ranjit, K.T. and K.J. Klabunde, Solvent effects in the hydrolysis of magnesium methoxide, and the production of nanocrystalline magnesium hydroxide. An aid in understanding the formation of porous inorganic materials. Chem. Mater., 2005. 17(1): p. 65-73.

10. Kessler, V.G., et al., New insight in the role of modifying ligands in the sol-gel processing of metal alkoxide precursors: A possibility to approach new classes of materials. J. Sol-Gel Sci. Technol., 2006. 40(2-3): p. 163-179.

11. Broussous, L., et al., SAXS Study of Formation and Growth of Tin Oxide Nanoparticles in the Presence of Complexing Ligands. J. Phys. Chem. B, 2002. 106(11): p. 2855-2860.

12. $\mathrm{Hu}, \mathrm{M} .-\mathrm{C}$. , et al., Probing the early-stage/rapid processes in hydrolysis and condensation of metal alkoxides. J. Mater. Sci., 2000. 35(8): p. 1957-1971.

13. Wilkinson, A.P., et al., In situ $x$-ray diffraction study of crystallization kinetics in PbZr1-xTixO3,(PZT, $x=0.0,0.55,1.0)$. Chem. Mater., 1994. 6(6): p. 750-754.

14. Fan, J., S.W. Boettcher, and G.D. Stucky, Nanoparticle assembly of ordered multicomponent mesostructured metal oxides via a versatile sol-gel process. Chem. Mater., 2006. 18(26): p. 6391-6396.

15. Baney, R.H., et al., Silsesquioxanes. Chem. Rev., 1995. 95(5): p. 1409-1430.

16. Lerouge, F., G. Cerveau, and R.J.P. Corriu, Supramolecular selforganization in non-crystalline hybrid organic-inorganic nanomaterials induced by van der Waals interactions. New J. Chem, 2006. 30(10): p. 1364-1376.

17. Gnanasekaran, D., K. Madhavan, and B. Reddy, Developments of polyhedral oligomeric silsesquioxanes (POSS), POSS nanocomposites and their applications: a review. J Sci Ind Res, 2009. 68(6): p. 437464.

18. Dalwani, M., et al., Ultra-thin hybrid polyhedral silsesquioxanepolyamide films with potentially unlimited 2D dimensions. J. Mater. Chem., 2012. 22(30): p. 14835-14838.

19. Raaijmakers, M.J.T., et al., Sieving of Hot Gases by Hyper-CrossLinked Nanoscale-Hybrid Membranes. Journal of the American Chemical Society, 2013. 136(1): p. 330-335. 
20. Moreau, J.J., et al., Self-organized hybrid silica with long-range ordered lamellar structure. J. Am. Chem. Soc., 2001. 123(32): p. 7957-7958.

21. Lerouge, F., G. Cerveau, and R.J.P. Corriu, Hydrophobic induced supramolecular self-organization of tetrahedral units based on van der Waals interactions. J Mater Chem, 2006. 16(1): p. 90-95.

22. Agirre, I., et al., Hybrid organosilica membranes and processes: Status and outlook. Sep. Purif. Technol., 2014. 121: p. 2-12.

23. Castricum, H.L., et al., Hybrid ceramic nanosieves: Stabilizing nanopores with organic links. Chem. Commun., 2008(9): p. 11031105.

24. Dubois, G., et al., Molecular Network Reinforcement of Sol-Gel Glasses. Advanced Materials, 2007. 19(22): p. 3989-3994.

25. Dubois, G., et al., Superior mechanical properties of dense and porous organic/inorganic hybrid thin films. J. Sol-Gel Sci. Technol., 2008. 48(1-2): p. 187-193.

26. Francisco, M.S.P., R. Landers, and Y. Gushikem, Local order structure and surface acidity properties of $a \mathrm{Nb}<$ sub $>2</$ sub $>0<$ sub $>5</$ sub $>/$ SiO $<$ sub $>2</$ sub $>$ mixed oxide prepared by the solgel processing method. J. Solid State Chem., 2004. 177(7): p. 24322439.

27. Bradley, D. and I. Thomas, 687. Organosilyloxy-derivatives of metals. Part I. Alkylsilyloxy-derivatives of titanium, zirconium, niobium, and tantalum. J. Chem. Soc. (Resumed), 1959: p. 34043411.

28. Ibers, J.A., Crystal and Molecular Structure of Titanium (IV) Ethoxide. Nature, 1963. 197(4868): p. 686-687.

29. Caruso, J., et al., Solvent-dependent ester elimination and ligand exchange reactions between trimethylsilyl acetate and tin (IV) tetratert-butoxide. Inorg. Chem., 1995. 34(2): p. 449-453.

30. Iwasaki, M., et al., Preparation of high homogeneity BaO-TiO2-SiO2 gel. J. Sol-Gel Sci. Technol., 1994. 2(1-3): p. 387-391.

31. Jansen, M. and E. Guenther, Oxide gels and ceramics prepared by a nonhydrolytic sol-gel process. Chem. Mater., 1995. 7(11): p. 21102114.

32. Caruso, J., et al., Ester elimination versus ligand exchange: the role of the solvent in tin-oxo cluster-building reactions. J. Chem. Soc., Chem. Commun., 1995(2): p. 157-158. 
33. Look, J.L. and C. Zukoski, Colloidal Stability and Titania Precipitate Morphology: Influence of Short-Range Repulsions. J. Am. Ceram. Soc., 1995. 78(1): p. 21-32.

34. Bansal, N.P., Low-temperature synthesis, pyrolysis and crystallization of tantalum oxide gels. J. Mater. Sci., 1994. 29(19): p. 5065-5070.

35. Gaudon, A., et al., Phase separation in sol-gel derived $\mathrm{ZrO2-SiO2}$ nanostructured materials. J. Eur. Ceram. Soc., 2005. 25(2-3 SPEC. ISS.): p. 283-286.

36. Scolan, E. and C. Sanchez, Synthesis and characterization of surfaceprotected nanocrystalline titania particles. Chemistry of Materials, 1998. 10(10): p. 3217-3223.

37. Blanchard, J., et al., Hydrolysis and Condensation Reactions of Transition Metal Alkoxides: Calorimetric Study and Evaluation of the Extent of Reaction. Eur. J. Inorg. Chem., 1998. 1998(8): p. 11151127.

38. Errington, R.J., et al., B-diketonate derivatives of titanium alkoxides: $X$-ray crystal structures and solution dynamics of the binuclear complexes $[\{T i \quad(O R)<$ sub $>3</ s u b>(d i k)\}<$ sub $>2</$ sub $>]$. Polyhedron, 1998. 17(5): p. 659-674.

39. Spijksma, G.I., et al., Stabilization and destabilization of zirconium propoxide precursors by acetylacetone. Chem. Commun., 2004. 10(16): p. 1874-1875.

40. In, M. and R. Prud'hornme, Fourier transform mechanical spectroscopy of the sol-gel transition in zirconium alkoxide ceramic gels. Rheologica acta, 1993. 32(6): p. 556-565.

41. Spijksma, G.I., et al., Nona-coordinated MO6N3 centers $\mathrm{M}=\mathrm{Zr}, \mathrm{Hf}$ as a stable building block for the construction of heterometallic alkoxide precursors. Inorg. Chim. Acta, 2007. 360(6): p. 2045-2055.

42. Spijksma, G.I., et al., Microporous Zirconia-Titania composite membranes derived from diethanolamine-modified precursors. Adv. Mater., 2006. 18(16): p. 2165-2168.

43. Puchberger, M., et al., Reaction of metal alkoxides with 3-alkylsubstituted acetylacetone derivatives-coordination vs. hydrodeacylation. New J. Chem., 2004. 28(11): p. 1289-1294.

44. Rupp, W., N. Hüsing, and U. Schubert, Preparation of silica-titania xerogels and aerogels by sol-gel processing of new single-source precursors. J. Mater. Chem., 2002. 12(9): p. 2594-2596.

45. Vioux, A., Nonhydrolytic sol-gel routes to oxides. Chem. Mater., 1997. 9(11): p. 2292-2299. 
46. Barnes, G.T. and I.R. Gentle, Interfacial Sciece. 2005, New York: Oxford University Press.

47. Brinker, C., et al., Sol-gel strategies for controlled porosity inorganic materials. J. Membr. Sci., 1994. 94(1): p. 85-102.

48. Raman, N., et al., Sol-gel strategies for amorphous inorganic membranes exhibiting molecular sieving characteristics. 1994, Sandia National Labs., Albuquerque, NM (United States).

49. Raman, N.K., M.T. Anderson, and C.J. Brinker, Template-based approaches to the preparation of amorphous, nanoporous silicas. Chem. Mater., 1996. 8(8): p. 1682-1701.

50. Bouwmeester, H.J.M. and A.J. Burggraaf, Chapter 10 Dense ceramic membranes for oxygen separation, in Membrane Science and Technology, A.J. Burggraaf and L. Cot, Editors. 1996, Elsevier. p. 435-528.

51. Lu, G.Q., et al., Inorganic membranes for hydrogen production and purification: A critical review and perspective. J. Colloid Interf. Sci., 2007. 314(2): p. 589-603.

52. Agirre, I., et al., Hybrid organosilica membranes and processes: Status and outlook. Sep. Purif. Technol., 2014. 121: p. 2-12.

53. Li, J.-R., et al., Carbon dioxide capture-related gas adsorption and separation in metal-organic frameworks. Coordin. Chem. Rev., 2011. 255(15-16): p. 1791-1823.

54. Ayral, A., et al., Microporous silica membrane: basic principles and recent advances. Membr. Sci. Technol., 2008. 13: p. 33-79.

55. Wee, S.-L., C.-T. Tye, and S. Bhatia, Membrane separation processPervaporation through zeolite membrane. Sep. Purif. Technol., 2008. 63(3): p. 500-516.

56. de Vos, R.M. and H. Verweij, Improved performance of silica membranes for gas separation. J. Membr. Sci., 1998. 143(1-2): p. 37-51.

57. Shekhawat, D., D.R. Luebke, and H.W. Pennline, A review of carbon dioxide selective membranes. US Department of Energy, 2003.

58. Xomeritakis, G., et al., Tubular ceramic-supported sol-gel silicabased membranes for flue gas carbon dioxide capture and sequestration. J. Membr. Sci., 2009. 341(1): p. 30-36.

59. Boffa, V., et al., Microporous niobia-silica membrane with very low CO2 permeability. ChemSusChem, 2008. 1(5): p. 437-443.

60. Qi, H., et al., Hybrid Organic-Inorganic Microporous Membranes with High Hydrothermal Stability for the Separation of Carbon Dioxide. ChemSusChem, 2010. 3(12): p. 1375-1378. 
61. Qi, H., et al., Effect of $\mathrm{Nb}$ content on hydrothermal stability of a novel ethylene-bridged silsesquioxane molecular sieving membrane for H2/CO2 separation. J. Membr. Sci., 2012. 421-422(0): p. 190200.

62. Kanezashi, M. and M. Asaeda, Hydrogen permeation characteristics and stability of Ni-doped silica membranes in steam at high temperature. J. Membr. Sci., 2006. 271(1-2): p. 86-93. 
Chapter 1 
Theory of small Angle X-ray Scattering 
By convention, the scattered intensity is described as a function of the scattering vector $\mathbf{q}$. The $\mathbf{q}$-vector describes the displacement of the scattered wave with respect to its original trajectory. When particles are isotropically dispersed the intensity averages out over all possible orientations. Consequently, the intensity becomes only dependent on the length of the q-vector, the $q$-value. The $q$-value is independent of the azimuthal angle in the plane of the detector and only depends on the scattering angle $\theta$, as described by the equation:

$$
q=4 \pi \cdot \sin (\theta) / \lambda
$$

Unlike $\theta$ the $q$-value is characteristic for a particular feature size and does not depend on the wavelength $\lambda$. When interpreting SAXS data, a system can be characterized either as a particulate system or a non-particulate system [1, 2]. A particulate system is characterized as an assembly of particles with a certain shape in a particular arrangement. Its scattering intensity function $I(q)$ is described as the product of two separate functions: (1) the form function $P(q)$ that describes the characteristic scattering intensity arising from intraparticle correlations is only dependent on the size and shape of particles, and (2) the structure function $S(q)$ that arises from inter-particle correlations is solely dependent on how particles are arranged or agglomerated with respect to each other. The intensity function for particulate systems is described by [1-3]:

$$
I(q)=N \cdot(\Delta \rho)^{2} \cdot S(q) \cdot P(q)
$$

Herein, $N$ represents the number of particles in the beam and $\Delta \rho$ represents the average difference in electron density between particles and their surroundings. Some systems, such as bicontinuous systems cannot be interpreted as an assembly of particles. The intensity function of such nonparticulate systems cannot be described by separate form and structure functions. These systems are further discussed in section 2.4. 


\subsection{Form function}

The form function $P(q)$ describes the characteristic scattering intensity of isolated particles. In the case of well dispersed particles at relatively low concentrations, mutual particle interference is negligible and the intensity function $I(q)$ is solely described by a form function i.e. $S(q)=1$. SAXS from isolated particles arises from the fact that the electron density inside particles is larger than the electron density of the surrounding medium. Consequently, X-rays have an enlarged probability to scatter inside a particle rather than outside. The scattered intensity correlates with the probability that two waves scatter simultaneously at a particular vector $\mathbf{r}$ separated from each other. Therefore, when the probability of finding two locations within a particle for all possible separation vectors $\mathbf{r}$ (i.e. the socalled correlation function $\boldsymbol{\gamma}(\boldsymbol{r})$ ) is known, its corresponding intensity function can be derived by a conversion of $\gamma(\boldsymbol{r})$ to reciprocal space through the Fourier transform [1-3]:

$\mathbf{I}(\mathbf{q})=\int_{V} \gamma(\mathbf{r}) \cdot \exp (i \cdot \mathbf{q} \cdot \mathbf{r}) d V$

For instance, for an isolated sphere with a radius $R=1$ and a homogeneous electron distribution, a normalized electron density profile can be defined as 1 inside the sphere and 0 outside the sphere. When placing the origin in the center of the sphere, the electron density profile is described by Figure 2.1a. Its corresponding $\gamma(\boldsymbol{r})$ (Figure 2.1b) is defined as the convolution product of the electron density profile with itself averaged over its volume. At a length of $0, \gamma(r)$ is 1 by definition [4] and with increasing length of $r$, the number of possibilities of placing vector $r$ inside a sphere decreases. $\boldsymbol{\gamma}(\boldsymbol{r})$ becomes zero when the length of $r$ becomes larger than the diameter of the sphere $(2 R)$. The determination of $\boldsymbol{\gamma}(\boldsymbol{r})$ through the convolution integral is usually omitted by calculation of the amplitude function $\mathbf{A}(\mathbf{q})$ (Figure 2.1d) by the Fourier transform [1, 2]:

$$
\mathbf{A}(\mathbf{q})=\int_{V} \Delta \mathbf{\rho}(\mathbf{r}) \cdot \exp (i \cdot \mathbf{q} \cdot \mathbf{r}) d V
$$


The intensity function is easily derived from $\mathbf{A}(\mathbf{q})$ as the square of its modulus (Figure 1e). Note that for asymmetric particles such as droplet shaped particles the amplitude function is complex while the intensity function is always real and positive. For isotropic particles such as spheres the Fourier transform can be simplified to an integral of scalar elements:

$$
A(q)=4 \pi \cdot \int_{0}^{\infty} \Delta \rho(r) \cdot r^{2} \cdot \frac{\sin (q \cdot r)}{(q \cdot r)} d r
$$

In the case of an isolated anisotropic particle, such as a cylinder or a plate its scattering intensity is essentially anisotopic as well. Yet, when particles are randomly dispersed inside a particular medium, the scattering intensity averages out over all possible orientations and the measured $I(q)$ becomes isotropic. Combined with the loss of the imaginary component of the amplitude functions, the intensity function has substantially less information when the particles become randomly oriented. Therefore, when $I(q)$ is measured its corresponding electron density distribution function cannot be derived unambiguously. Instead, assumptions should be made concerning possible particles and $I(q)$ can be fitted through iterative processes. By convention, the part of the amplitude $A(q)$ and intensity $I(q)$ functions that only describe intra-particle correlations are represented by $F(q)$ and $P(q)$, respectively, and weighted over $V_{p}$ and $V_{p}{ }^{2}$, where $V_{p}$ is the particle volume. Consequently, both $F(q)$ and $P(q) \rightarrow 1$ for $q \rightarrow 0$. A variety of different form functions have been derived and a clear overview was given by Pedersen [5]. 


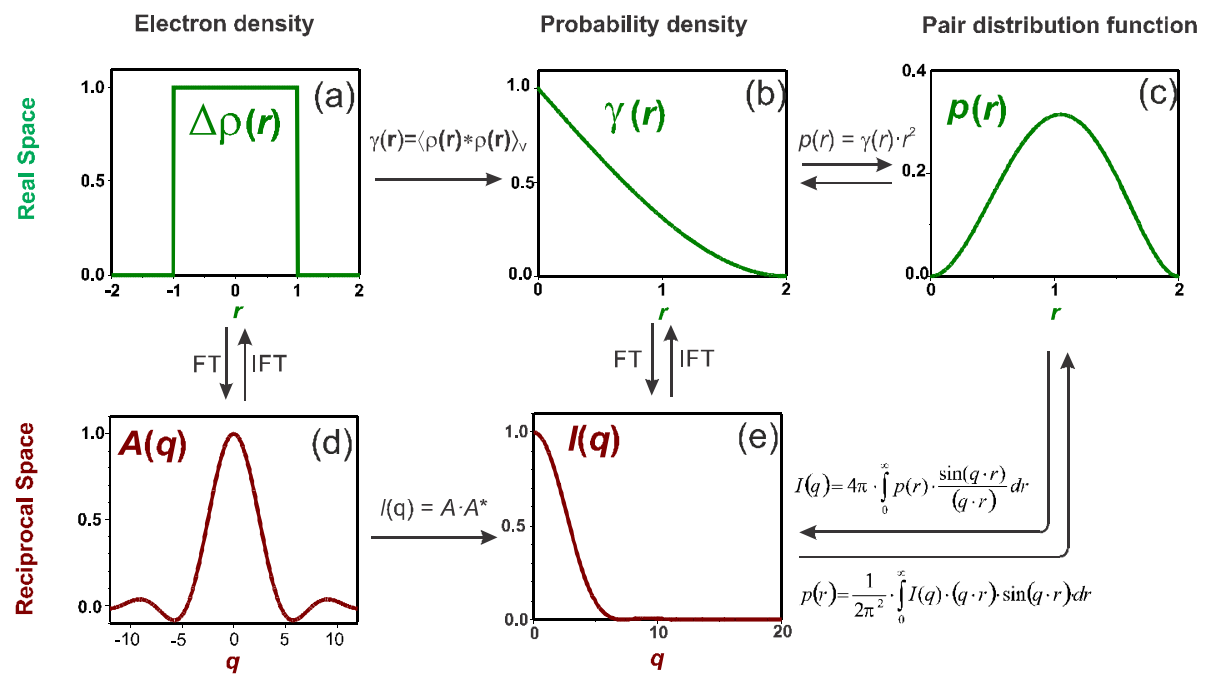

Figure 2.1 A scheme that illustrates the relationships between the relevant functions in the analysis of SAXS data, using an isolated sphere with $r_{0}=1$ as example. (a) the electron density distribution function, (b) the (auto)correlation function, (c) the pair distance distribution function, (d) the amplitude function, (e) the scattering intensity function. Note that both $A(q), \mathcal{X r}$ ) and $I(q)$ are normalized over the volume of a sphere.

Instead of fitting the experimental data, the data can be converted to a pair (distance) distribution function $p(r)$ (Figure 2.1c). This function is closely related to $\mathcal{X}(r)$, however in the case of $\mathcal{X}(r)$ any vector $r$ in all possible spherical angles $\phi$ and $\theta$ are weighted evenly regardless of the length of $\mathbf{r}$. On the other hand, $p(r)$ also encounters for the fact that the probability of finding correlation within the imaginary sphere described by $r$ scales with the surface of this imaginary sphere $4 \pi \cdot r^{2}$. Therefore, $p(r)$ is scaled by $r^{2}$ (Figure $2.1 \mathrm{~b}-\mathrm{c}$ ). The advantage of determining $p(r)$ is that particular features give better distinguishable features as compared to $I(q)$. For instance hollow particles result in truncated curves of $p(r)$ and for increasing elongation of a particle $p(r)$ becomes more tailed $[1,2]$.

\subsection{Structure function}

The structure function is a part of the intensity function that arises from constructive scattered waves that are originating from different particles. This function is a representation of the probability that a particular particle is surrounded by another particle. For isotropic systems structure factors 
can be divided in two type of systems: (1) correlated systems that can describe packing of colloidal particles and (2) polymeric systems that can be described by either fractalic or wormlike models. By convention, the structure function is weighted over the square of the particle volume of the repetitive unit (known as the primary particle), such that $S(q \rightarrow \infty)=1$.

\subsubsection{Correlated systems}

When increasing the concentration of a colloid, particles will start to interfere with each other and the scattered intensity cannot be described solely by the form function. The simplest case is the interference between hard non-sticky spheres. Their interference pattern is solely determined by the impossibility that two hard spheres of radius $R_{\mathrm{HS}}$ interpenetrate. In other words, the repulsive potential between two spheres is infinite when the radial distance $r<2 \cdot R_{\mathrm{HS}}$ and becomes zero when $r>2 \cdot R_{\mathrm{HS}}$. For very dilute systems the probability that two particles collide is negligible. Thus, the location of particles is random and no interparticle interference is observed. With increasing concentration, the probability that spheres collide increases and the probability of finding two particles at a separation distance $\sigma=$ $2 \cdot R_{\mathrm{HS}}$ becomes larger. Hence, a correlation peak appears in the intensity spectrum at $q \sim 2 \pi / \sigma$.

The solution of the structure factor of hard non-sticky spheres relies on the equation of sate of non-ideal gases with a limited compressibility [6]. One of the most straightforward solutions was given by Fournet, who used a Born-Green Approximation (Equation 2.6, Table 2.1). The model works reasonably well and has been applied several times, but at high concentrations it underestimates the extent of higher order correlations, i.e. correlations that arise from the third and a fourth sphere interacting with two other spheres [6-9]. At higher packing densities an extended formula is needed, based on a Percus-Yevick approximation (Equation 2.7, Table 2.1) [10-12]. This approach have been applied for colloidal packing of a variety of different nanocomposites [10-16]. 


\section{Table 2.1 Structure functions of correlated spheres}

\begin{tabular}{|c|c|c|}
\hline Definition / Energy potential & Structure function & $\mathrm{Nr} / \mathrm{ref}$ \\
\hline $\begin{array}{l}\text { Born Green Approximation: } \\
U_{\mathrm{HS}}\left(r, R_{\mathrm{S}}\right)=\left\{\begin{array}{lll}\infty & \text { for } & r \leq \sigma \\
0 & \text { for } & r>\sigma\end{array}\right.\end{array}$ & $S(q, \sigma)=\frac{1}{1+8 \cdot \phi \cdot \frac{\sin (q \cdot \sigma)-q \cdot \sigma \cdot \cos (q \cdot \sigma)}{(q \cdot \sigma)^{3}}}$ & $\begin{array}{l}(2.6) \\
{[6-9]}\end{array}$ \\
\hline $\begin{array}{l}\text { Hard non-sticky sphere } \\
\text { Percus-Yevic Approximation } \\
U_{\mathrm{HS}}\left(r, R_{\mathrm{S}}\right)=\left\{\begin{array}{lll}\infty & \text { for } & r \leq \sigma \\
0 & \text { for } & r>\sigma\end{array}\right.\end{array}$ & $\begin{array}{l}S(q, \sigma)={\frac{1}{1+24 \cdot(\phi / K) \cdot\left(\alpha \cdot Y_{1}(K)+\beta \cdot Y_{2}(K)+\gamma \cdot Y_{4}(K)\right)}}^{K=q \cdot \sigma, \quad \alpha=\frac{(1+2 \phi)^{2}}{(1-\phi)^{4}}, \quad \beta=-6 \cdot \phi \cdot \frac{(1+\phi / 2)^{2}}{(1-\phi)^{4}}, \quad \gamma=\frac{\phi \cdot \alpha}{2}}\end{array}$ & $\begin{array}{r}(2.7) \\
{[10-12]}\end{array}$ \\
\hline$U_{\mathrm{sW}}\left(r, R_{\mathrm{S}}\right)=\left\{\begin{array}{ccc}\infty & \text { for } & r \leq \sigma \\
-\varepsilon & \text { for } & \sigma<r \leq \lambda \cdot \sigma \\
0 & \text { for } & r>\lambda \cdot \sigma\end{array}\right.$ & $\begin{array}{l}S(K)=\frac{1}{1+24 \cdot(\phi / K) \cdot C(K)} \quad \text { where: } \\
C(K)=\alpha \cdot Y_{1}(K)+\beta \cdot Y_{2}(K)+\left(\frac{\phi \cdot \alpha}{2}\right) \cdot Y_{4}(K)-\left(\frac{\varepsilon}{k_{B} \cdot T}\right) \cdot F_{\varepsilon}(K)^{(\#)} \\
F_{\varepsilon}(K)=\left(\frac{\sin (\lambda \cdot K)-\lambda \cdot K \cdot \cos (\lambda \cdot K)}{K^{2}}-Y_{1}(K)\right), \quad K=q \cdot \sigma \\
\alpha=\frac{(1+2 \phi)^{2}+\phi^{3} \cdot(\phi-4)}{(1-\phi)^{4}}, \quad \beta=-\left(\frac{\phi}{3}\right) \cdot \frac{\left(18+20 \cdot \phi-12 \cdot \phi^{2}+\phi^{4}\right)}{(1-\phi)^{4}}\end{array}$ & (2.8) \\
\hline $\begin{array}{l}\text { Hard sticky sphere with } \\
\text { Baxter potential based on PY } \\
\text { approximation }\end{array}$ & $\begin{array}{l}S(K)=\frac{1}{1-C(K)} \quad \text { where: } \\
C(K)=\frac{2 \cdot \phi \cdot \lambda}{K} \cdot \sin (K)-2\left(\frac{\phi \cdot \lambda}{K}\right)^{2} \cdot(1-\cos (K))-\left(\frac{24 \cdot \phi}{K}\right) \cdot F_{\varepsilon}(K)\end{array}$ & (2.9) \\
\hline & $\begin{array}{l}F_{\varepsilon}(K)=\left(\alpha \cdot Y_{1}(K)+\beta \cdot Y_{2}(K)+\left(\frac{\alpha \cdot \phi}{2}\right) \cdot Y_{4}(K)\right), \quad K=q \cdot \sigma \\
\alpha=\frac{\left(1+2 \phi-\mu^{2}\right)}{(1-\phi)^{4}}, \quad \beta=-\left(\frac{3 \phi \cdot(2+\phi)^{2}-2 \mu \cdot\left(1+7 \phi+\phi^{2}\right)+\mu^{2} \cdot(2+\phi)}{2 \cdot(1-\phi)^{4}}\right) \\
\gamma=\frac{\phi \cdot(2+\phi)}{6 \cdot(1-\phi)^{2}}, \quad \varepsilon=\tau+\frac{\phi}{1-\phi}, \quad \lambda=\frac{6}{\phi} \cdot\left(\varepsilon-\sqrt{\varepsilon^{2}-\gamma}\right), \quad \mu=\lambda \cdot \phi \cdot(1-\phi)\end{array}$ & \\
\hline \multicolumn{3}{|c|}{$\begin{array}{ll}\text { (\#) With standard integrals } \boldsymbol{Y}_{\mathrm{n}}(\boldsymbol{K}) \text { : } & \\
Y_{\mathrm{n}}(K)=\int_{0}^{1} x^{n} \cdot \sin (K \cdot x) d x & Y_{1}(K)=\frac{\sin (K)-K \cdot \cos (K)}{(K)^{2}}, Y_{2}(K)=\frac{2 \cdot K \cdot \sin (K)+\left(2-K^{2}\right) \cdot}{(K)^{3}} \\
\text { that correspond to: } & Y_{4}(K)=\frac{\left(-K^{4}+12 K^{2}-24\right) \cdot \cos (K)+4\left(K^{3}-6 K\right) \cdot \sin (K)+24}{(K)^{5}}\end{array}$} \\
\hline
\end{tabular}


In real systems, such as nanocomposites [12, 14, 20-23], ionomers [10] and microemulsions $[24,25]$, electron dense particles are often separated by electron lean regions. The electron lean regions often stabilize the electron dense particles. Such systems can be modeled as core-shell particles, as illustrated in Figure 2.2a. When such particles are dispersed in a solvent it is essentially a ternary system. However, if the difference in electron density between solvent and electron lean regions is sufficiently small, the scattering by the electron lean regions may be neglected and such systems are reduced to binary systems, with a form factor with core radius $R_{\mathrm{C}}$ and structure function with a separation distance $\sigma=2 \cdot R_{\mathrm{HS}}=2 \cdot\left(R_{\text {core }}+R_{\text {Shell }}\right)$.

An example of such a system with $S(q)=(2.7$ and $P(q)=$ Guinier approximation is shown Figure $2.2 \mathrm{~b}$ for varying volume fractions $\phi$. With increasing volume fraction the following phenomena are observed: (1) The height of the correlation peak increases as the number of correlations between spheres increases, (2) the correlation peak becomes narrower as the free volume in which the sphere can move reduces and the packing of spheres becomes more rigid, and (3) the intensity at low- $q$ value decreases due to a decrease in contrast in electron density between particles and their surroundings, since the surroundings contain increasing numbers of particles. Note that the latter statement is only valid for systems that contain non-sticky spheres, where long-range order is absent. However, if for instance the Van der Waals forces are sufficiently strong, particles assemble either temporarily or permanently into larger agglomerates and next-nearest-neighbor correlations (second, third, etc.) are observed. These systems are well described by structure functions that include a small attractive well, such as spheres with a square well potential [17] and Baxter Sticky spheres $[18,19]$, as described by Equation (2.8) and (2.9) respectively (Table 2.1). 


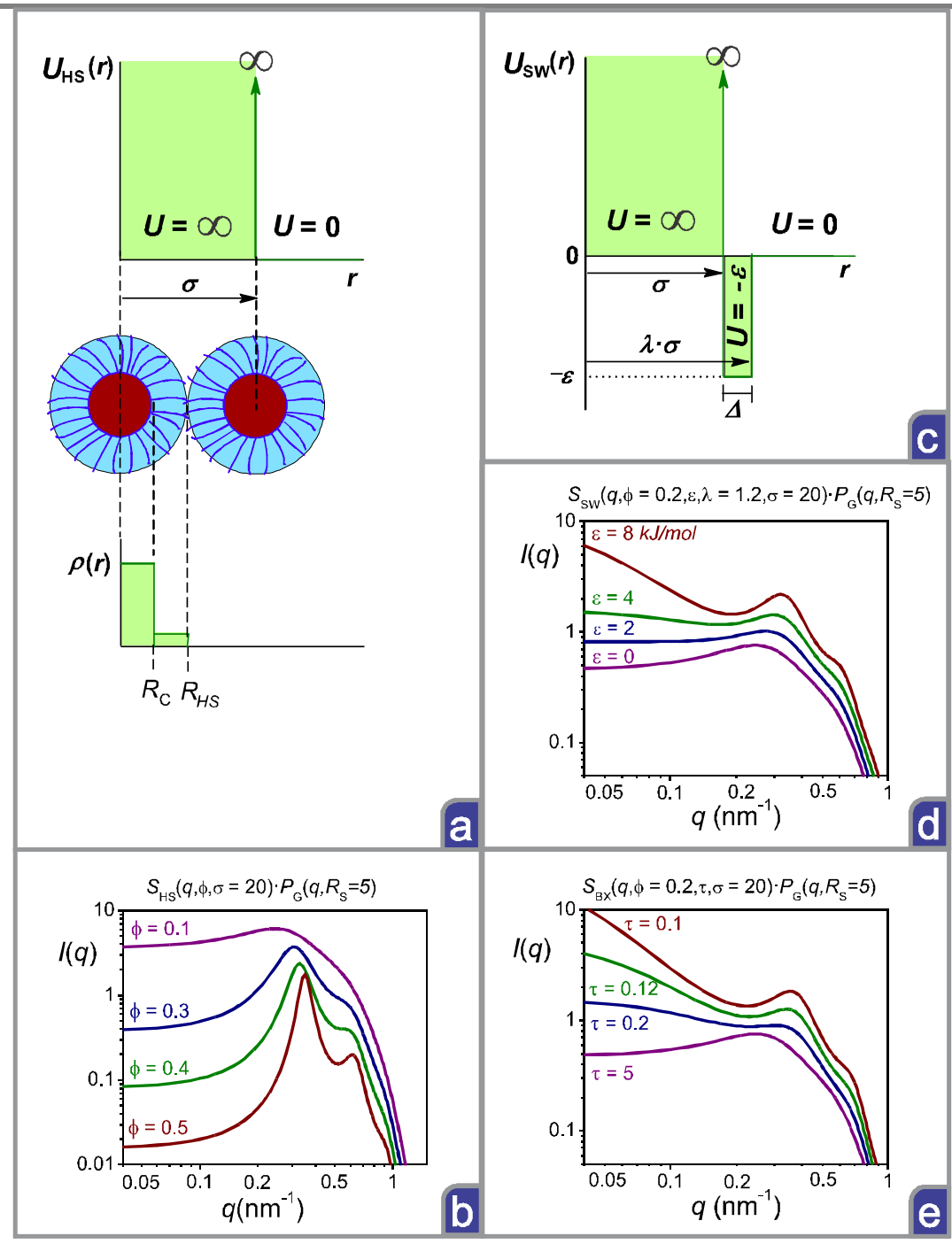

Figure 2.2. (a) Illustration of spherical agglomerates with a core-shell electron density distribution, separated at a distance $\sigma=\mathbf{2} \boldsymbol{R}_{\mathrm{HS}}$ (b) Simulated $I(q)$ following a hard sphere packing potential, with varying volume fractions $\phi$ and a constant $\sigma=20 \mathrm{~nm}$. (c) Illustration of hard sphere packing with a square well potential of depth $\varepsilon$ in between $\sigma$ and $\lambda \cdot \sigma$. (d) Simulated $I(q)$ of packed with a square well potential and varying depths $\varepsilon$, for $\phi=0.2, \sigma=20 \mathrm{~nm}$ and $\lambda=1.2$. (e) Simulated $I(q)$ of packed spheres following an attractive Baxter potential with varying reduced temperatures $(\tau)$ of an infinity small attractive layer, with $\phi=0.2$ and $\sigma=20 \mathrm{~nm}$. For all simulations (b, $d, e)$ the form function of the core was described by a Guinier approximation of spheres with a cross-sectional radius $R_{\mathrm{C}}=5 \mathrm{~nm}\left(\boldsymbol{R}_{\mathrm{G}}=\right.$ $3 n m)$. 
In the case of a square well potential, between a minimum radial separation distance $\sigma$ and a distance $\lambda \cdot \sigma$, particles experience a mutual attractive force $\varepsilon$ while they do not interact beyond that point $(U=0)$ as illustrated in Figure 2.2c [17]. The extent of correlations between particles increases with increasing depth $\varepsilon$ and increasing length $\Delta=\sigma \cdot(\lambda-1)$ of the well. Contrary to the case of non-sticky spheres, not only the direct particleparticle correlations increase with $\mathcal{E}$, as observed by the increasing intensity of the correlation peak (Figure 2.2d), but next-nearest neighbor (second, third, etc.) correlations increase as well, as evidenced by an increasing intensity in the low- $q$ regime. Note that next-nearest neighbor correlations are most pronounced at relatively low volume fractions of particles $\phi$, since small agglomerates are easily distinguishable from their surroundings. At higher $\phi$ the agglomerates approach each other and next-nearest neighbor correlations are diminished by longer distance correlations, and the intensity in the low-q-regime drops. Meanwhile, due to an increasing number of next-nearest neighbor correlations the correlation peak becomes more intense, similar to non-sticky spheres. The square well model has been applied for a variety of systems including colloidal nanoparticles [26-29], micro-emulsions [25] and micelles [30].

In analogy with the square well model of Baxter, sticky spheres also introduce an adhesive force beyond a separation distance $\sigma$. However, unlike the square well potential the length of the well is defined as infinitely small, i.e. $\Delta \rightarrow 0$ so that adhesion only depends on the reduced temperature $\tau$, which is essentially a ratio between the actual temperature and a certain critical temperature of a system. Therefore, $\tau$ is a measure of the mobility of particles in the system and decreases with increasing adhesive force between particles. As $\tau$ decreases, an increasing amount of particles will agglomerate and consequently the scattering intensity of the low- $q$ regime and the correlation peak increases (Figure 2.2e). The use of a single parameter that characterized the particle adhesion is more straightforward from an analysis point of view, since $\Delta$ is often unknown and a particular value should be assumed. On the other hand, the square well potential was found to give more realistic scattering curves $[29,31]$. 


\subsubsection{Mass-fractal structure functions}

Small particles may agglomerate in a random fashion forming small branched assemblies. Depending on the reaction kinetics, for instance whether a reaction is rate limited or diffusion limited, particles assemble in varying morphologies and packing densities [32, 33]. Such randomly packed systems may be described as mass fractals. When a number of small building blocks with radius $r_{0}$ is assembled as a massfractal, the expected number of building blocks inside an imaginary sphere with radius $r$ is given by $[34,35]$ :

$$
N(r)=\left(\frac{r}{r_{0}}\right)^{D}
$$

Here, the dimensionality $D$ is related to the packing density of the agglomerate. A particle can only be regarded as a mass fractal when the building blocks are packed so that the system is self-similar over at least one order of magnitude. A system is referred to as self-similar when a particular way that particles are packed together on a small length scale is similar to the way that particles are packed on a larger scale. As illustrated in Figure 3, the red circles were packed inside a blue dotted circle similar to the way that the blue dotted circles were packed inside the green dotted circle. For fractalic systems the packing of blue dotted circles inside the green dotted circle does not necessarily need to be an exact replica of the red balls inside the blue dotted circle. As long as the average packing dimensionality remains constant over different radii $r$ within a certain size range, it obeys Equation (2.10) and the system can be referred as a fractal. Note that the blue dotted circle, which is one order of magnitude larger as the red circle contains 29 red circles and the green dotted circle, which is one order of magnitude large as the blue dotted circles contains 29 blue dotted circles. Therefore, the packing dimensionality remain constant at $D$ $={ }^{10} \log (29)=1.46$ for two orders of magnitude. 


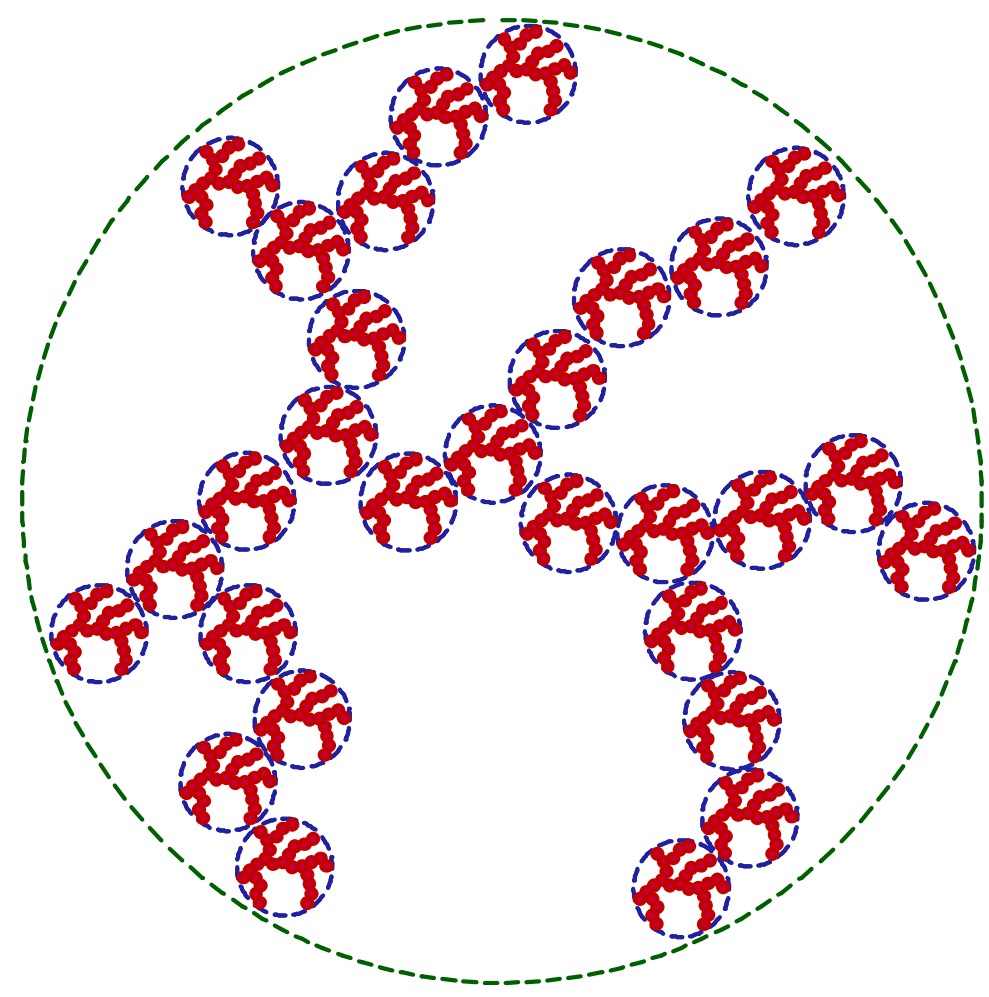

Figure 3. A conceptual illustration of a self-similar agglomerate. The blue dotted circles inside the green dotted circle is self-similar compared to the red circles inside the blue dotted circle. Both are consistent with $D={ }^{10} \log (29)=1.46$.

Its pair distance distribution function $g_{0}(r)$ describes the probability of finding a correlation at given $r$, while the cumulative distribution $N(r)$ Equation (2.10) describes the probability of a correlation within a sphere of radius $r$. Consequently, the pair correlation function $g_{0}(r)$ is described by a derivative of $N(r)$ :

$g_{0}(r)=\frac{1}{4 \pi \cdot r^{2}} \cdot \frac{d N}{d r}=\frac{D}{4 \pi \cdot N_{p}} \cdot \frac{r^{D-3}}{r_{0}}$

Here, $N_{\mathrm{p}}$ is the total number of particles and $g_{0}(r)$ is closely related to $\chi(r)$. However, it only considers pairwise interactions between two different particles. The function $g_{0}(r)((2.12)$ represents an fractal agglomerate with 
an infinite size and a cut-off function was introduced to define the finite agglomerate size. When introducing an exponential cut-off function exp($r / \xi)$, the relation for pairwise interactions given by Zernike and Prins [36] was solved analytically [34, 35]:

$$
S_{M F}\left(q, D, \xi, r_{0}\right)=1+\frac{D \cdot \Gamma(D-1) \cdot \sin [(D-1) \cdot \arctan (q \cdot \xi)]}{\left(q \cdot r_{0}\right)^{D} \cdot\left(1+\frac{1}{(q \cdot \xi)^{2}}\right)^{\left(\frac{D-1}{2}\right)}}
$$

This structure function is useful to describe a variety of systems including some sol-gels. However, in particular cases, for instance when growth is controlled by diffusion limited cluster aggregation, these aggregates were found to be less polydisperse as an exponential cutoff function implies. In these cases sharper cutoff functions are more realistic, as explained in more detail by Sorensen and Wang [33]. Moreover, we derived a new fractalic model with a variable Schultz-Zimm distributed cutoff as it is described in more detail in the next chapter (Chapter 3).

\subsection{Polydisperse systems}

If the form function of a monodisperse system is known, then the form function of a polydisperse system is [1]:

$$
P_{D}(q, x)=\frac{\int_{0}^{\infty} D(x, \mu) \cdot P(q, x) \cdot m(x)^{p} d x}{\int_{0}^{\infty} D(x) \cdot m(x)^{p} d x}=\frac{\int_{0}^{\infty} D(x) \cdot P(q, x) \cdot x^{p \cdot d} d x}{\int_{0}^{\infty} D(x) \cdot x^{p \cdot d} d x}
$$

where $x$ is a particular size parameter, $P_{D}(q, x)$ and $P(q, x)$ are the form functions of a polydisperse and a monodisperse system, respectively. $D(x, \mu)$ is a probability distribution function that also depends on the average size $\mu ; m$ is a mass term and $p$ is an exponent that depends on the type of distribution. Since the scattering intensity scales with the square of the mass or volume of electrons that are involved, a number, volume and intensity weighted average are weighted by $p=2,1$ and 0 , respectively. The mass term, whichin the case of SAXS is proportional to the number of 
electrons, scales with $x^{d}$, where $d$ represents the number of dimensions in which the size parameter $x$ is applied. For instance, in the case that $x$ represents the length of a cylinder $d=1$, when $x$ represents the radius of a cylinder $d=2$, and when $x$ represents the radius of a sphere $d=3$.

When interparticle correlations are involved, the calculation of the intensity function becomes more complex since it involves essentially all interactions between particle one with size $x_{1}$ and particle 2 with size $x_{2}$, particle 3 with size $x_{3}$, etc. Fortunately, these systems can be approximated by the local monodisperse approximation (LMA), which assumes that particles are only surrounded by other particles of the same size [37]. Following this approximation the corresponding scattering intensity can be described as:

$$
\begin{aligned}
& I_{D}(q, x)=N \cdot(\Delta \rho)^{2} \cdot \frac{\int_{0}^{\infty} D(x, \mu) \cdot P(q, x) \cdot S(q, x) \cdot m(x)^{p} d x}{\int_{0}^{\infty} D(x, \mu) \cdot m(x)^{p} d x} \\
& I_{D}(q, x)=N \cdot(\Delta \rho)^{2} \cdot \frac{\int_{0}^{\infty} D(x, \mu) \cdot P(q, x) \cdot S(q, x) \cdot x^{p \cdot d} d x}{\int_{0}^{\infty} D(x, \mu) \cdot x^{p \cdot d} d x}
\end{aligned}
$$

\subsection{Non-particulate systems}

Some systems, such as bicontinuous systems cannot be interpreted as an assembly of particles. Instead, these systems can be analyzed by statistical models that describe characteristic fluctuations in the electron density. A useful model for bicontinuous systems was proposed by Teubner and Strey. It characterizes electron density fluctuations by two characteristic length scales, namely a correlation length $\xi$ and a domain scale length $d$ [38]:

$$
I(q)=\frac{8 \pi \cdot V \cdot c_{2} \cdot \Delta \rho^{2}}{\xi \cdot\left(a_{2}+c_{1} \cdot q^{2}+c_{2} \cdot q^{4}\right)}
$$


where:

$$
\begin{aligned}
& \xi=\frac{1}{\sqrt{\left(1 / 2 \cdot \sqrt{\left(a_{2} / c_{2}\right)}+1 / 4 \cdot\left(c_{1} / c_{2}\right)\right)}} \text { and: } \\
& \frac{d}{2 \pi}=\frac{2 \pi}{\sqrt{\left(1 / 2 \cdot \sqrt{\left(a_{2} / c_{2}\right)}-1 / 4 \cdot\left(c_{1} / c_{2}\right)\right)}}
\end{aligned}
$$

This expression combines an expression of randomly sized spheres with a characteristic $\xi$ with a expression that has a quasi-periodic repeat distance $d$. In analogy with Debye's equation of randomly distributed overlapping spheres [39], the specific surface area of one phase in another phase is inversely proportional to the specific surface area: $S / V=4 \phi \cdot(1-\phi) / \xi$, were $S / V$ is a surface to volume ratio and $\phi$ is the volume fraction of either one of both phases in a two-phase system. This equation allows the description of various microemulsions ranging from ordered lamellas $(\xi>>d)$, until randomly sized spheres $(\xi<<d)[24,38]$. An increasing $\xi / d$ ratio corresponds to an increasing extent of order, which corresponds to a more pronounced and narrower correlation peak in the intensity profile. This approach has been extended for more complex systems as extensively discussed by Antony Roberts [40].

\subsection{References}

1. Feigin, L.A. and D.I. Svergun, Structure analysis by small-angle $x$-ray and neutron scattering 1987, New York: Plenum Press.

2. Glatter, O. and O. Kratky, Small-angle x-ray scattering 1982, London: Academic Press.

3. Craievich, A., Small Angle X-ray Scattering by Nanostructured Materials, in Handbook of Sol-Gel Science and Technology; Processing Characterisation and Applications, S. Sakka and R.M. 
Almeida, Editors. 2005, Kluwer Academic Publishers: New York, Boston, Dordrecht, London, Moscow. p. 161-189.

4. Debye, P. and A. Bueche, Scattering by an inhomogeneous solid. J. Appl. Phys., 1949. 20(6): p. 518-525.

5. Pedersen, J.S., Analysis of small-angle scattering data from colloids and polymer solutions: modeling and least-squares fitting. Adv. Colloid Interfac., 1997. 70(0): p. 171-210.

6. Guinier, A. and G. Fournet, General Theory, in Small-Angle Scattering of X-rays1955, John Wiley \& Sons: New York. p. 5-82.

7. Yarusso, D.J. and S.L. Cooper, Microstructure of ionomers: interpretation of small-angle $x$-ray scattering data. Macromol., 1983. 16(12): p. 1871-1880.

8. Dahmouche, K., et al., Nanostructure and luminescent properties of sol-gel derived europium-doped amine functionalised hybrids. J. Solgel Sci. Technol., 2006. 37(2): p. 99-104.

9. Beaucage, G. and D.W. Schaefer, Structural studies of complex systems using small-angle scattering: a unified Guinier/power-law approach. J. Non-Cryst. Solids, 1994. 172-174, Part 2(0): p. 797805.

10. Kinning, D.J. and E.L. Thomas, Hard-sphere interactions between spherical domains in diblock copolymers. Macromol., 1984. 17(9): p. 1712-1718.

11. Ashcroft, N.W. and J. Lekner, Structure and Resistivity of Liquid Metals. Phys. Rev., 1966. 145(1): p. 83-90.

12. Terrill, N.J., et al., Small-angle X-ray scattering studies on colloidal dispersions of polyaniline-silica nanocomposites. Langmuir, 1993. 9(8): p. 2093-2096.

13. Manet, S., et al., Structure of micelles of a nonionic block copolymer determined by SANS and SAXS. J. Phys. Chem. B, 2011. 115(39): $p$. 11318-11329.

14. Besselink, R., et al., Evolution of microstructure in mixed niobiahybrid silica thin films from sol-gel precursors. J. Colloid Interf. Sci., 2013. 404(0): p. 24-35.

15. Stawski, T.M., et al., Development of nanoscale inhomogeneities during drying of sol-gel derived amorphous lead zirconate titanate precursor thin films. Langmuir, 2011. 27(17): p. 11081-11089.

16. Stawski, T.M., et al., Nanostructure development in alkoxidecarboxylate-derived precursor films of barium titanate. J. Phys. Chem. C, 2012. 116(1): p. 425-434. 
17. Sharma, R.V. and K.C. Sharma, The structure factor and the transport properties of dense fluids having molecules with square well potential, a possible generalization. Physica A, 1977. 89(1): p. 213-218.

18. Menon, S.V.G., V.K. Kelkar, and C. Manohar, Application of Baxter's model to the theory of cloud points of nonionic surfactant solutions. Phys. Rev. A, 1991. 43(2): p. 1130-1133.

19. Baxter, R., Percus-Yevick equation for hard spheres with surface adhesion. J. Chem. Phys., 1968. 49: p. 2770.

20. Gaudon, A., et al., Phase separation in sol-gel derived $\mathrm{ZrO2-SiO2}$ nanostructured materials. J. Eur. Ceram. Soc., 2005. 25(2-3 SPEC. ISS.): p. 283-286.

21. Gaudon, A., et al., From amorphous phase separations to nanostructured materials in sol-gel derived $\mathrm{ZrO2}: \mathrm{Eu} 3+/ \mathrm{SiO} 2$ and ZnO/SiO2 composites. J. Non-Cryst. Solids, 2006. 352(21-22): p. 2152-2158.

22. Rodrigues, D.E., et al., Structural Features of Sol-Gel derived hybrid Inorganic Organic Network Ceramer Materials by Small-Angle X-rayScattering. Chem. Mater., 1992. 4(6): p. 1437-1446.

23. Santilli, C., et al., Controlled Drug Release from Ureasil- Polyether Hybrid Materials. Chem. Mater., 2009. 21(3): p. 463-467.

24. Schubert, K.-V., et al., Small angle neutron scattering near Lifshitz lines: Transition from weakly structured mixtures to microemulsions. J. Chem. Phys., 1994. 101(6): p. 5343-5355.

25. Fisher, S., et al., Solubilization of simvastatin and phytosterols in a dilutable microemulsion system. Colloid Surface B, 2013. 107, p. 3542.

26. Orts-Gil, G., et al., Characterisation of silica nanoparticles prior to in vitro studies: from primary particles to agglomerates. J Nanopart. Res., 2011. 13(4): p. 1593-1604.

27. Graf, P., et al., Peptide-Coated Silver Nanoparticles: Synthesis, Surface Chemistry, and $\mathrm{pH}$-Triggered, Reversible Assembly into Particle Assemblies. Chem. Eur. J., 2009. 15(23): p. 5831-5844.

28. Pontoni, D., et al., Interactions and kinetic arrest in an adhesive hard-sphere colloidal system. J. Chem. Phys., 2003. 119: p. 6157.

29. Sztucki, M., et al., Kinetic arrest and glass-glass transition in shortranged attractive colloids. Phys. Rev. E, 2006. 74(5): p. 051504.

30. Simmons, B., et al., Small Angle Neutron Scattering Study of Mixed AOT + Lecithin Reverse Micelles. Langmuir, 2002. 18(22): p. 83458349. 
31. Dawson, K., et al., Higher-order glass-transition singularities in colloidal systems with attractive interactions. Phys. Rev. E, 2000. 63(1): p. 011401.

32. Brinker, C.J.S., G. W. , Sol Gel Science, Phys. Chem. Sol-Gel Processing. Vol. 1. 1990, San Diego: Acedemic Press. 1-881.

33. Sorensen, C.M. and G.M. Wang, Size distribution effect on the power law regime of the structure factor of fractal aggregates. Phys. Rev. E, 1999. 60(6): p. 7143-7148.

34. Chen, S.H. and J. Teixeira, Structure and fractal dimension of protein-detergent complexes. Phys. Rev. Lett., 1986. 57(20): p. 2583-2586.

35. Teixeira, J., Small-angle scattering by fractal systems. J. Appl. Crystallogr., 1988. 21(6): p. 781-785.

36. Zernike, F.t. and J. Prins, Die beugung von roentgenstrahlen in fluessigkeiten als effekt der molekuelanordnung. Zeitschrift für Physik, 1927. 41(6-7): p. 184-194.

37. Pedersen, J., Determination of size distribution from small-angle scattering data for systems with effective hard-sphere interactions. J. Appl. Crystallogr., 1994. 27(4): p. 595-608.

38. Teubner, M. and R. Strey, Origin of the scattering peak in microemulsions. J. Chem. Phys., 1987. 87(5): p. 3195-3200.

39. Debye, P., H. Anderson, and H. Brumberger, Scattering by an inhomogeneous solid. II. The correlation function and its application. J. Appl. Phys., 1957. 28(6): p. 679-683.

40. Roberts, A.P., Statistical reconstruction of three-dimensional porous media from two-dimensional images. Phys. Rev. E, 1997. 56(3): p. 3203-3212. 


\section{Rapid early stage Schultz-Zimm distributed mass-fractalic growth studies on niobia/ silsesquioxiane mixtures}




\subsection{Abstract}

Rapid in-situ SAXS measurements were performed to measure early-stage agglomeration processes under a continuous flow of niobium ethoxide mixtures. The growth characteristics of niobium ethoxide were characterized in the presence and absence of a silsesquioxane 1,2-bis(triethoxysilyl)-ethane (BTESE) at $T=25$ and $T=40^{\circ} \mathrm{C}$. Data was analysed with a newly derived mass-fractlic model. Unlike the conventional exponential cutoff model, a variable cutoff function in the correlation function was being introduced without introducing laborious numerical calculations. An increasing sharpness of the cutoff function was associated with an decreasing polydispersity

The analysed data both in the presence and absence of BTESE revealed rather monodisperse selpreserving polydispersities that were associated with diffiusion limited cluster agglomeration (DLCA). In the absence of BTESE growth could be characterized by Smolucowski type agglomeration kernels with a homogeneity parameter $\lambda \sim 0$. Nevertheless, in the presence of BTESE the growth exponents became substantially smaller $(p<0.2$, with $\left.R_{\mathrm{G}} \sim t^{p}\right)$. The reduced growth exponent could not be described by irreversible Smoluchowski type kernels and was more consistent with reversible Lifshitz-Slyozov type agglomeration kernels. The growth of the niobia clusters was clearly suppressed by the presence of BTESE.

\subsection{Introduction}

Organically bridged silsesquioxanes exhibit very high hydrothermal, acid and mechanical stability, which is substantially larger than $\mathrm{SiO}_{2}$ [1-5]. Therefore, it can withstand very harsh conditions, which make it potentially suitable as a carrier for catalytic materials. It can also exhibit micropores, when processed properly, suitable for filtration and pervaporation or for membrane reactors [2-7]. To widen its applicability to other molecular separation processes and catalysis, bridged silsesquioxanes may be altered by doping them with transition metal oxides. Hence, selective transition 
metal centres are created that may be useful either for catalytic purposes or to alter the molecular diffusivity and/or adsorption in these materials. For instance, the introduction of nickel, cobalt, zirconium and niobium oxides in silica and hybrid silica membranes was found to enhance the $\mathrm{H}_{2} / \mathrm{N}_{2}$ and $\mathrm{H}_{2} / \mathrm{CO}_{2}$ permselectivity [8-14]. Nevertheless, homogeneous distribution of metal oxides within a matrix of bridged silsesquioxanes is not straightforward from a synthetic point of view. The metal oxide tends to be introduced either as metal salt, or as a metal alkoxides that tends to phase separate while the networks forms. Metal salts do not contribute to the gel network of the silsesquioxane, and are therefore poorly compatible with these networks and simply phase separate as metal salts. Metal alkoxides do form gels, however their reactivity is known to be orders of magnitude higher than that of silicon alkoxides [15-17]. Optimization of such mixed systems is often a matter of trial and error. Nevertheless, we may find more systematic solutions by gaining more insight into the kinetics of such evolving systems.

Much is known concerning the kinetics of silicon alkoxides, which because of their relatively low reactivities allow monitoring by a large number of characterization techniques. These include ${ }^{29} \mathrm{Si}-\mathrm{NMR}$, which not only monitors the number of specific chemical groups, but also the number of specific groups that are coordinating the silicon atom [18-21]. It allows the identification of different reaction rate constants depending on the chemical nature of the Si atom. In the case of metal alkoxides NMR cannot be used due to the very fast kinetics of these materials [15-17], combined with the complex nature of their quadrupolar nuclei [22]. Consequently, only a limited number of kinetic studies on metal alkoxides were found in literature which rely on other techniques including Small Angle X-ray Scattering (SAXS), Fourier Transform Infrared Spectroscopy (FTIR), electron conductivity and dynamic light scattering (DLS) [23-28]. Alternatively, macroscopic effects including turbidity and geltime have often been used to predict effects on the molecular level [29-31]. However, on a macroscopic additional effects play a role, such as micellar stabilization. Hence, the kinetics on microscopic level are not always directly correlated with the kinetics on molecular level [16]. 
The extremely fast early stage reaction kinetics of titanium and zirconium alkoxides were already well described by the work of Harris et al. [21, 23]. In the work presented here we focused on the nucleation and growth of niobium penta-ethoxide, and the extent to which growth is influenced by the presence of a bridged silsesquioxane, i.e. 1,2-bis-(triethoxysilyl)-ethane (BTESE). Niobia was proven to be a useful dopant either for scavenging $\mathrm{CO}_{2}$ or as Lewis acid catalyst [12, 32-34]. The early stages of these growth kinetics were monitored by time-resolved Synchrotron Small Angle X-ray Scattering (SAXS) experiments. This approach not only allows us to determine the agglomerate sizes, but also their shape and polydispersity in $5 \mathrm{~s}$ timeframes.

\subsection{Experimental Section}

\subsubsection{Recipe mixing experiments}

All precursor solutions were prepared inside a glovebox under nitrogen atmosphere. The synthesis was performed under atmospheric conditions. Firstly, a mixed metal alkoxide precursor solution was made in dry ethanol which contained $0.635 \mathrm{~mol} / \mathrm{L} \mathrm{1,2-bis-triethoxysilyl-ethane} \mathrm{(BTESE)} \mathrm{sol} \mathrm{and}$ $0.318 \mathrm{~mol} / \mathrm{L}$ niobium pentaethoxide (NPE, ABCR chemicals, 99.99\%). This corresponds to a $[\mathrm{Si}]:[\mathrm{Nb}]$ ratio of $4: 1$ and an overall ethoxide concentration $[-O E t]=5.4 \mathrm{~mol} / \mathrm{L}$, i.e. including the ethoxide groups of both BTESE and NPE. Secondly, diluted nitric acid solutions were prepared which contained $\left[\mathrm{H}_{2} \mathrm{O}\right]=3.857 \mathrm{~mol} / \mathrm{L}$ and $\left[\mathrm{HNO}_{3}\right]=0.129 \mathrm{~mol} / \mathrm{L}$ in dry ethanol. By mixing an equal volume of the combined alkoxide precursor solution and the diluted nitric acid solution a hydrolysis ratio $\left[\mathrm{H}_{2} \mathrm{O}\right] /[(\mathrm{Si})-\mathrm{OEt}]=1$, an acid ratio $\left[\mathrm{HNO}_{3}\right] /[(\mathrm{Si})-\mathrm{OEt}]=1 / 30$ and $[-\mathrm{OEt}]=2.7 \mathrm{~mol} / \mathrm{L}$ was reached. Particle growth in these solutions was monitored at $T=25^{\circ} \mathrm{C}$ and $T=40^{\circ} \mathrm{C}$, and these samples are further referred to as $\mathrm{SiNbT} 25$ and SiNbT40, respectively.

Experiments were also performed in the absence of BTESE. NPE precursor solutions with a concentration of $0.318 \mathrm{~mol} / \mathrm{L} \mathrm{Nb}$ were prepared. Similarly to the mixtures with BTESE exactly the same diluted nitric acid solutions were used and also mixed in a equal volume ratio with the NPE precursor solution. Particle growth in these mixtures was also monitored at $T=25^{\circ} \mathrm{C}$ 
and $T=40^{\circ} \mathrm{C}$, and these samples are further referred to as NbT25 and NbT40, respectively.

Heated flow cell

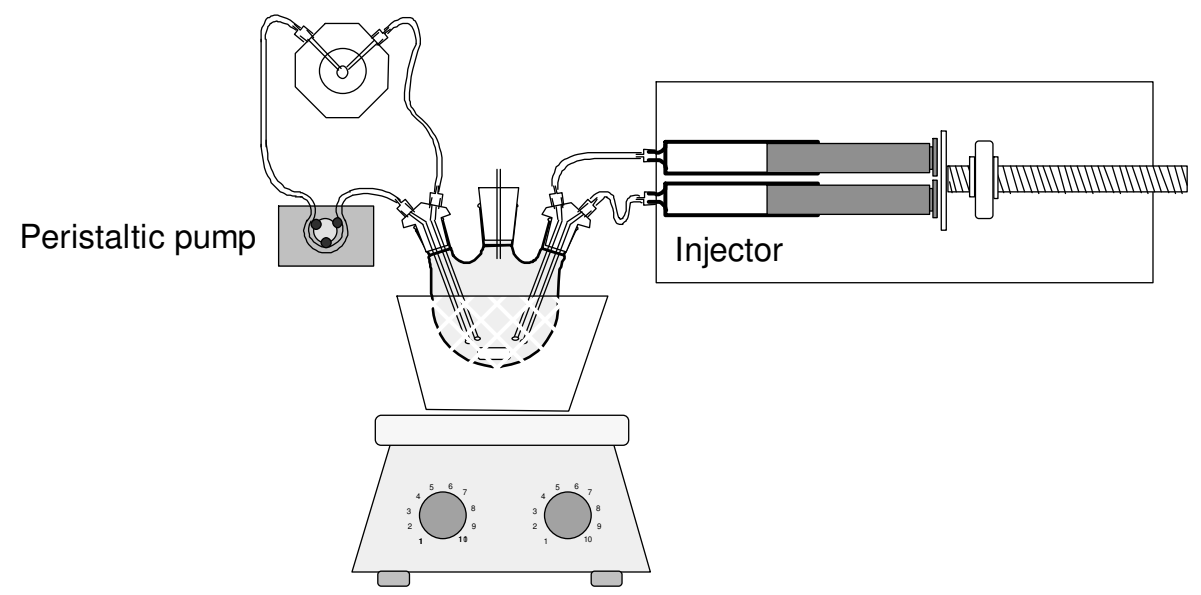

Heated oil bath

Figure 3.1. Schematic diagram of mixing setup used in SAXS experiments.

\subsubsection{Mixing Setup}

SAXS mixing experiments were executed with a setup as illustrated in Figure 3.1. A homemade flowcell was used that consisted of an aluminium housing with luerlock connectors for connecting tubes. The cell consisted of two Kapton foils that were compressed against an aluminium interior by two aluminium rings with sealing rubbers outside the cell. The cell itself was cylindrical with a depth of $1 \mathrm{~mm}$ and a cross-sectional diameter of $5 \mathrm{~mm}$. Continuous flow between the flow cell and the reactor that consisted of a $25 \mathrm{~mL}$ three-neck round-bottom flask was arranged by a Masterflex peristaltic pump. The dead time between the round-bottom flask and the flow cell was 10 s. Before mixing the solutions rapid SAXS-measurements were already started to ensure that measurement was immediately done when mixing the solutions. Then, the measurement was initiated by a timer 
that started the injector. In $8 \mathrm{~s}$ the injector injected both $12 \mathrm{~mL}$ of diluted nitric acid solution and $12 \mathrm{~mL}$ of metal alkoxide solution simultaneously into the three-neck round-bottom flask. The round-bottom flask contained a small vent to release overpressure that was formed during injection.

\subsubsection{Time-resolved SAXS experiments}

Small-angle X-ray scattering was carried out using synchrotron radiation on the Dutch-Belgian beamline, DUBBLE BM-26B of the ESRF in Grenoble [35]. The $X$-ray beam with an energy of $12 \mathrm{keV}$ was focused on a corner of the $2 \mathrm{D}$ Pilatus $1 \mathrm{M}$ CCD-detector to maximize the covered range of scattering angles. A beam stop was applied to shield the detector from the direct beam and avoid saturation of the outgoing signal. The detector was placed at a distance of $1.36 \mathrm{~m}$ from the sample, which resulted in a measurable $q$ range: $0.17<q<6.00 \mathrm{~nm}^{-1}$. All scattering data were found to be independent of the scattering angle in the plane of the detector, which indicated that particles were isotropic or isotropically dispersed throughout the sample. Therefore, all channels with the same $q$ value were averaged with each other. Silver behenate was used as calibration standard for the determination of the absolute scale of the scattering vector $q$ in our experiments [36].

All curves were normalized by dividing the scattering intensity by the time of data acquisition and the signal of the ionization chamber in front of the sample. A background subtraction procedure was carried out. For sols, the scattering signal of a capillary filled with ethanol was subtracted. 


\subsection{Theoretical background}

\subsubsection{Characterization of SAXS data}

\subsection{1a. Mass-fractal agglomerates with an exponential cut-off length $\xi$}

After a short hydrolysis period, small clusters of BTESE and NPE nucleated and subsequently agglomerated into amorphous assemblies of these primary clusters. In analogy with previous reported SAXS-experiments on mixed BTESE/NPE sols [37], these agglomerates could be well described in terms of mass fractals. Due to shadow effect of the vacuum tubing that was used to reduce scattering from air between the sample and the detector, the measurements were only reliable up to $q=6 \mathrm{~nm}^{-1}$. Consequently, the transition from the fractal regime to the Porod regime could not be observed and the radii of the primary nuclei could not be determined. This obstacle was circumvented by using an alternative scattering intensity function for mass fractals as described by Sorensen [38]:

$$
S_{\mathrm{EC}}\left(q, D_{\mathrm{f}}, \xi\right)=\frac{\sin \left(\left(D_{\mathrm{f}}-1\right) \cdot \operatorname{atan}(q \cdot \xi)\right)}{\left(D_{\mathrm{f}}-1\right) \cdot q \cdot \xi \cdot\left(1+(q \cdot \xi)^{2}\right)^{\left(\frac{D_{\mathrm{f}}-1}{2}\right)}}
$$

Herein, $D_{\mathrm{f}}$ describes the dimensionality of mass fractals, and $\xi$ the typical cut-off distance that determines the finite size of an agglomerate. This intensity function is closely related to the structure function of mass fractals as described by Teixeira $[39,40]$. However, the radius of the primary scatters or nuclei $\left(r_{0}\right)$ is assumed to be infinitely small (i.e. $\lim r_{0} \rightarrow 0$ ), in contrast to the mass fractal description of Teixeira, where $r_{0}$ has a finite value. Therefore, Equation (3.1) cannot be normalized over the volume of the primary scatterer. Instead, it is normalized over the volume of the whole agglomerate as determined by $\xi$. Note that the $\xi$-parameter is 
introduced as an exponentially decaying function $(h(r, \xi)=\exp (-r / \xi))$ in the pair correlation function for 3-dimensional agglomerates:

$\gamma\left(r, D_{\mathrm{f}}, \xi\right)=r^{D_{\mathrm{f}}-3} \cdot h(r, \xi)$

The typical volume (Porod volume) of such an agglomerate is described by $[41,42]$ :

$V_{\mathrm{A}}=\int_{0}^{\infty} 4 \cdot \pi \cdot r^{2} \cdot \gamma(r) d r=4 \pi \cdot \Gamma\left(D_{\mathrm{f}}\right) \cdot \xi^{D_{\mathrm{f}}}$

where $\Gamma$ represented the gamma function. Since Equation (3.1) was normalized over its entire agglomerate volume, it is being normalized as a form function and it obeys $S(q \rightarrow 0)=1$. Throughout, the $q$-region $P(q)$ was set to 1 and the overall scattering intensity was described by:

$$
I(q)=I_{0} \cdot S(q)
$$

where $I_{0}=N \cdot\left(V_{A}\right)^{2}$, which corresponds to the scattering intensity at $q \rightarrow 0$ (since $S(q \rightarrow 0)=1), N$ is the particle number density and $V_{\mathrm{A}}$ the particle volume of the fractalic agglomerate (Equation (3.3)). Note that the ratio $I_{0} /$ $V_{\mathrm{A}}=N \cdot V_{\mathrm{A}} \cdot\left(\Delta \rho^{2}\right)$ is proportional to the mass concentration of agglomerates in the solution, while the contrast in electron density $(\Delta \rho)$ is presumebly constant during agglomeration. The radius of gyration of these agglomerates can be extracted when $D_{\mathrm{f}}$ and $\xi$ are known through the equation:

$$
R_{\mathrm{G}}=\xi \cdot \sqrt{\frac{D_{\mathrm{f}} \cdot\left(D_{\mathrm{f}}+1\right)}{2}}
$$




\subsection{1b. Mass-fractal agglomerates with a Schultz-Zimm distributed cut-off length.}

As discussed by Sorensen [38] the diffusion limited cluster aggregation (DLCA) mechanism typically leads to less polydisperse agglomerates as implied by the exponentially decaying cut-off function. Sharper cut-off functions such as a Gaussian cutoff $\left(h(r, \xi)=\exp \left(-(r / \xi)^{2}\right)\right.$ were found to be more realistic for a variety of different aggregation mechanisms. On the other hand, it would be convenient to define a function where the sharpness of the cutoff function could be related to the degree of polydispersity. Therefore, firstly we introduce an infinitely sharp cut-off function; i.e. a unit step or Heaviside step function $h(r, \xi)=H(\xi-r)$. The intensity function of a mass-fractal with a hard cutoff function was described by an rotationally averaged Fourier transform:

$S_{\mathrm{HC}}(q, \xi)=\frac{4 \pi}{q \cdot V_{\mathrm{A}}} \int_{0}^{\infty} H(\xi-r) \cdot r^{D_{\mathrm{f}}-2} \cdot \sin (q \cdot r) d r=\frac{4 \pi}{q \cdot V_{\mathrm{A}}} \int_{0}^{\xi} r^{D_{\mathrm{f}}-2} \cdot \sin (q \cdot r) d r$

Herein, $H(\xi-r)$ represents the unit step function. $H(\xi-r)=1$ for $r<\xi$ and $H(\xi-$ $r)=0$ for $r>\xi . V_{\mathrm{p}}$ represents the volume of the agglomerate. Instead of using the unit step function we can also move the upper boundary of the sine transform from $\infty$ to $\xi$, as shown in the right hand side part of Equation (3.6). Secondly, polydispersity is introduced by the integral:

$$
S_{\mathrm{SC}}(q, \xi)=\int_{0}^{\infty} w(\xi) \cdot I_{\mathrm{hf}}(q, \xi) d \xi
$$


Herein, $w(\xi)$ is an intensity weighted probability density function of the cutoff parameter $\xi$. We applied a Schultz-Zimm distribution [43] which was found to give realistic results in earlier studies on similar systems [37, 4446]:

$w(\xi, \mu, z)=\frac{a^{Z+1}}{\Gamma(Z+1)} \cdot \xi^{Z} \cdot \exp (-a \cdot \xi)$

where

$a=\frac{Z+1}{\mu}$

and $\mu$ is the intensity weighted average of $\xi$ and the $Z$-parameter is related to de distribution of the cutoff distance, i.e.: the variance of $\xi$ corresponds to $\left(\sigma_{\xi}\right)^{2}=\mu^{2} /(Z+1)$. By combining Equation (3.6), Equation (3.7) and Equation (3.8) we obtain:

$S_{\mathrm{SC}}\left(q, a, D_{\mathrm{f}}, Z\right)=\frac{4 \pi \cdot a^{Z+1}}{q \cdot V_{\mathrm{P}} \cdot \Gamma(Z+1)} \cdot \int_{0}^{\infty}\left[\xi^{Z}\left(\int_{0}^{\xi} r^{D_{\mathrm{f}}-2} \cdot \sin (q \cdot r) d r\right) \cdot \exp (-a \cdot \xi)\right] d \xi$

This integral can be evaluated as a Laplace transform and for integer values of $Z$ an analytical solution is given by:

$$
S_{\mathrm{SCI}}\left(q, a, D_{\mathrm{f}}, \zeta\right)=\frac{4 \pi \cdot a^{Z+1}}{2 i \cdot q \cdot V_{\mathrm{P}} \cdot \Gamma(Z+1)} \cdot(-1)^{Z} \cdot \frac{d^{Z}}{d a^{Z}}\left(\frac{1}{a} \cdot\left(\frac{1}{(a-i \cdot q)^{D-1}}-\frac{1}{(a+i \cdot q)^{D-1}}\right)\right)
$$

where $i$ is the imaginary number. The derivatives of $a$ that are expanding with increasing $Z$-value can be generalized by the following Riemann's sum: 


$$
S_{\mathrm{SC}}\left(q, a, D_{\mathrm{f}}, \zeta\right)=\frac{4 \pi}{2 i \cdot q \cdot V_{\mathrm{P}}} \cdot \sum_{\eta=0}^{Z}\left(\frac{\Gamma\left(D_{\mathrm{f}}+\eta-1\right)}{\Gamma(\eta+1)} \cdot a^{\eta} \cdot\left(\frac{1}{(a-i \cdot q)^{D_{\mathrm{f}}+\eta-1}}-\frac{1}{(a+i \cdot q)^{D_{\mathrm{f}}+\eta-1}}\right)\right)
$$

Herein, $\eta$ is an integer variable that is varies from 0 to $Z$. In analogy with the mass-fractal structure function with an exponential cutoff ((3.1), the function was normalized over its agglomerate volume $V_{\mathrm{A}}$ such that $S(q \rightarrow 0)=$ 1. The Porod volume of such agglomerate is described by:

$$
V_{\mathrm{A}}=4 \pi \cdot\left(\frac{a^{Z+1}}{\Gamma(Z+1)}\right) \cdot \int_{0}^{\infty} \xi^{Z}\left(\int_{0}^{\xi} r^{D_{\mathrm{f}}-3} \cdot r^{2} d r\right) \cdot \exp (-a \cdot \xi) d \xi
$$

which corresponded to:

$$
V_{\mathrm{A}}=\frac{4 \pi \cdot \Gamma\left(D_{\mathrm{f}}+Z+1\right)}{D_{\mathrm{f}} \cdot \Gamma(Z+1)} \cdot\left(\frac{\mu}{Z+1}\right)^{D_{\mathrm{f}}}
$$

Then, after normalization of Equation (3.11) with Equation (3.13), and replacing complex elements with goniometric equations we obtain:

$$
S_{\mathrm{SC}}\left(q, a, D_{\mathrm{f}}, Z\right)=\left(\frac{a}{q}\right) \cdot \frac{D_{\mathrm{f}} \cdot \Gamma(Z+1)}{\Gamma\left(D_{\mathrm{f}}+Z+1\right)} \cdot \sum_{\eta=0}^{Z}\left(\frac{\Gamma\left(D_{\mathrm{f}}+\eta-1\right)}{\Gamma(\eta+1)} \cdot \frac{\sin \left(\left(D_{\mathrm{f}}+\eta-1\right) \cdot \operatorname{atan}\left(\frac{q}{a}\right)\right)}{\left(1+\left(\frac{q}{a}\right)^{2}\right)^{\left(\frac{\left(D_{\mathrm{f}}+\eta-1\right)}{2}\right)}}\right)
$$


Now, let us define $\zeta$ as the integer part of $Z$ and $\phi$ as the fractional part of $Z$. Then, the contribution of the $\phi$ to $S_{\mathrm{sc}}(q)$ may be approximated as a $\phi$ fraction of the $\eta=\zeta+1$ element of the Riemann's Sum. Consequently the structure function for all values of $Z=\zeta+\phi$ can be approximated by:

$$
S_{\mathrm{SC}}\left(q, \mu, D_{\mathrm{f}}, Z\right)=\frac{D_{\mathrm{f}} \cdot \Gamma(Z+2)}{(q \cdot \mu) \cdot \Gamma\left(D_{\mathrm{f}}+Z+1\right)} \cdot\left(S_{\mathrm{I}}+\phi \cdot S_{\mathrm{F}}\right)
$$

where:

$$
S_{\mathrm{I}}=\sum_{\eta=0}^{\zeta}\left(\frac{\Gamma\left(D_{\mathrm{f}}+\eta-1\right)}{\Gamma(\eta+1)} \cdot \frac{\sin \left(\left(D_{\mathrm{f}}+\eta-1\right) \cdot \operatorname{atan}\left(\frac{q \cdot \mu}{Z+1}\right)\right)}{\left(1+\left(\frac{q \cdot \mu}{Z+1}\right)^{2}\right)^{\left(\frac{\left(D_{\mathrm{f}}+\eta-1\right)}{2}\right)}}\right)
$$

and:

$$
S_{\mathrm{F}}=\frac{\Gamma\left(D_{\mathrm{f}}+\zeta\right)}{\Gamma(\zeta+2)} \cdot \frac{\sin \left(\left(D_{\mathrm{f}}+\zeta\right) \cdot \operatorname{atan}\left(\frac{q \cdot \mu}{Z+1}\right)\right)}{\left(1+\left(\frac{q \cdot \mu}{Z+1}\right)^{2}\right)^{\left(\frac{\left(D_{\mathrm{f}}+\zeta\right)}{2}\right)}}
$$

where $S_{I}$ and $S_{F}$ represent the contributions of the integer and fractional values of $Z$ to $S_{s c}(q)$. For comparison of the Schultz cut-off model with the exponential cutoff model it is more convenient to express the size of a cluster by the radius of gyration that is derived from [41, 42]: 


$$
\left(R_{\mathrm{G}}\right)^{2}=\frac{1}{2} \cdot \frac{\int_{0}^{\infty} r^{4} \cdot \gamma(r) d r}{\int_{0}^{\infty} r^{2} \cdot \gamma(r) d r}
$$

that corresponds to:

$$
\left(R_{\mathrm{G}}\right)^{2}=\frac{1}{2} \cdot \frac{\int_{0}^{\infty} \xi^{z}\left(\int_{0}^{\xi} r^{4} \cdot r^{D_{\mathrm{f}}-3} d r\right) \cdot \exp (-a \cdot \xi) d \xi}{\int_{0}^{\infty} \xi^{z}\left(\int_{0}^{\xi} r^{2} \cdot r^{D_{\mathrm{f}}-3} d r\right) \cdot \exp (-a \cdot \xi) d \xi}
$$

which solution is given by:

$$
R_{\mathrm{G}}=\left(\frac{\mu}{Z+1}\right) \cdot \sqrt{\frac{1}{2} \cdot \frac{D_{\mathrm{f}} \cdot\left(D_{\mathrm{f}}+Z+1\right) \cdot\left(D_{\mathrm{f}}+Z+2\right)}{\left(D_{\mathrm{f}}+2\right)}}
$$

Since $\mathcal{X}(r)$ is essentially an auto-convolution product of $\Delta \rho(r)$ a hard cutoff function is not realistic. Therefore, the relative variance of $\xi$ that can be derived from the $Z$ parameter is always larger, because relative variance of $R_{G}$ and the relationship between them depends on the geometry of the fractal. Alternatively, we may extract a polydispersity factor $C_{P}$ following the procedure described by Sorensen and Wang [38]. Provided that $S(q)$ is normalized over the entire agglomerate volume Equation (3.13), such that $S(q \rightarrow 0)=1$, the effective structure function in the fractal regime $\left(q \cdot R_{G}>>1\right)$ is described by:

$$
S_{\text {eff }}\left(q, R_{\mathrm{G}}\right)=C \cdot C_{\mathrm{P}} \cdot\left(q \cdot R_{\mathrm{G}}\right)^{-D_{\mathrm{f}}} \quad \text { for }: q \cdot R_{\mathrm{G}}>>1
$$


where the constant $C$ is related to the geometry of the fractalic agglomerate and experimental results revealed that $C=1.0 \pm 0.05$ for mass fractals with $D_{f}$ in a range between 1.7 and 2.1 [38]. $C_{P}$ is a measure of the polydispersity and is defined by:

$C_{\mathrm{P}}=\frac{M_{1}}{M_{2}} \cdot\left(\frac{M_{\left(2+2 / D_{\mathrm{f}}\right)}}{M_{2}}\right)^{\left(D_{\mathrm{f}} / 2\right)}$

where $M_{\mathrm{j}}$ represents the $\mathrm{j}^{\text {th }}$ moment of the particle size distribution, and is defined as:

$$
M_{\mathrm{j}}=\int_{0}^{\infty}\left(N_{\mathrm{pa}}\right)^{j} \cdot n_{\mathrm{pa}}\left(N_{\mathrm{pa}}\right) \cdot d N_{\mathrm{pa}}
$$

Here $N_{\mathrm{pa}}$ represents the number of primary particles per agglomerate and $n_{\mathrm{pa}}\left(N_{\mathrm{pa}}\right)$ represents the particle number distribution function of primary particles per agglomerate. The $C_{P}$ value increases with increasing polydispersity and it can be associated with a particular growth mode, i.e. $C_{P} \sim 1.5$ for diffusion limited cluster aggregation (DLCA) and $C_{P}>2$ for reaction limited cluster aggregation (RLCA) [38]. $C_{P}$ is a size independent measure of polydispersity and solely depends on $Z$ and $D_{\mathrm{f}}$, and it can be derived from Equation (3.14) by taking the limit $\left(q \cdot R_{\mathrm{G}}\right) \rightarrow \infty$ of $S_{\mathrm{sc}}\left(q \cdot R_{\mathrm{G}}\right) \cdot\left(q \cdot R_{\mathrm{G}}\right)^{D f}$, which corresponds to:

$C \cdot C_{\mathrm{P}}=\sin \left[\left(D_{\mathrm{f}}-1\right) \cdot \frac{\pi}{2}\right] \cdot\left(\frac{D_{\mathrm{f}} \cdot \Gamma\left(D_{\mathrm{f}}-1\right) \cdot \Gamma(Z+1)}{\Gamma\left(D_{\mathrm{f}}+Z+1\right)}\right) \cdot\left(\frac{1}{2} \cdot \frac{D_{\mathrm{f}} \cdot\left(D_{\mathrm{f}}+Z+1\right) \cdot\left(D_{\mathrm{f}}+Z+2\right)}{\left(D_{\mathrm{f}}+2\right)}\right)^{\left(\frac{D_{\mathrm{f}}}{2}\right)}$ 
Note that the Riemann's sum diminished since the limit was dominated by the $\eta=0$ element of the Riemann's sum. As illustrated in Figure 2 by simulations of $S_{\mathrm{SC}}\left(q, \mu, D_{\mathrm{f}}, Z\right)$ with $R_{\mathrm{G}}=10 \mathrm{~nm}$ and $D_{\mathrm{f}}=2$, the height of the fractal regime as characterized by $C \cdot C_{P}$ decreases with increasing $Z$ values.

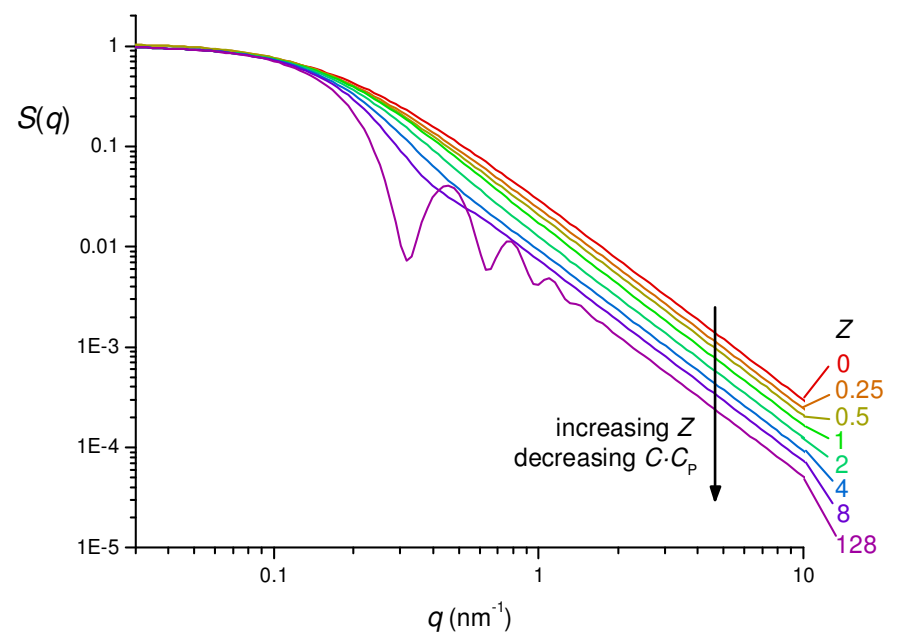

Figure 3.2. Simulated curves of $S_{\mathrm{SC}}\left(q, \xi, D_{\mathrm{f}}, Z\right)$, with $R_{\mathrm{G}}=10 \mathrm{~nm}$ and $D_{\mathrm{f}}=2$ being held constant and $z=0,0.25,0.5,1,2,4,8,128$, which corresponds to $C \cdot C_{\mathrm{p}}=3.00$, $2.45,2.10,1.67,1.25,0.93,0.73$ and 0.52 , respectively.

\subsubsection{Growth kinetics}

After a very short nucleation period growth is presumably accomplished by irreversible coagulation of primary nuclei which can by described by the Smoluchowski type agglomeration kernels [47-52]:

$R_{\mathrm{G}}(t)=\left(\sqrt[p]{R_{\mathrm{G}, t=0}}+k \cdot t\right)^{p}$

where: 
$p=\frac{1}{D_{\mathrm{f}}(1-\lambda)}$

Herein, $p$ is a growth exponent that determines the order of the reaction, $k$ is a reaction rate constant and $\lambda$ the homogeneity parameter. This equation includes a crossover regime between diffusion limited cluster aggregation (DLCA: $\lambda \sim 0$ ) and reaction limited cluster aggregation (RLCA: $\lambda \sim 1$ ) and is only valid for non-gelling agglomeration kernels $(\lambda<1)$. Moreover, these agglomeration kernels imply a self-preserving polydispersity. This means that depending on the growth limiting process (i.e. to what extent this is described by either DLCA or RLCA) a particular extent of polydispersity is preserved. The relation between $D_{\mathrm{f}}, \lambda$ and the polydispersity parameter $C_{\mathrm{P}}$ depends on the aggregation regime as determined by the Knudsen number $(\mathrm{Kn})$ [49]. Due to the interaction with solvent molecules in liquid media the mean free path of a particle is extremely small and $\mathrm{Kn} \sim 0$. Particles move by diffusion following the Stokes-Einstein relationship with a diffusion coefficient $D \sim R_{\mathrm{h}}{ }^{-1}$, where $R_{\mathrm{h}}$ is the hydrodynamic radius of a particle. In such a case $C_{\mathrm{p}}$ is described by [38]:

$C_{\mathrm{P}}\left(D_{\mathrm{f}}, \lambda\right)=\frac{1}{(2-\lambda)} \cdot\left[\frac{\Gamma\left(3-\lambda+2 / D_{\mathrm{f}}\right)}{\Gamma(3-\lambda)}\right]^{\left(D_{\mathrm{f}} / 2\right)}$

\subsection{Results and discussion}

As Figure 3.3 illustrates, the time resolved experiments of sample NbT25 could be described well by the Schultz cutoff function Equation (3.15) (colored lines). On the other hand, the exponential cutoff function (Equation (3.1), black lines) cannot follow the sharp curvature of the experimental data in the Guinier region. Consequently, the $D$ and $R_{\mathrm{G}}$ values obtained from the fits were systematically larger than obtained from the 
Schultz cutoff function. For instance, for $t=1 \mathrm{~min}$ with the Schultz cutoff: $Z$ $=6.3 \pm 0.7, D_{\mathrm{f}}=1.39 \pm 0.01$ and $R_{\mathrm{G}}=2.51 \pm 0.07 \mathrm{~nm}$, while for the exponential cutoff $D_{f}=1.93 \pm 0.04$ and $R_{\mathrm{G}}=2.92 \pm 0.07 \mathrm{~nm}$. In view of the goodness of the fit to the experimental data, the Schultz cutoff model was therefore considered more suitable for these datasets.

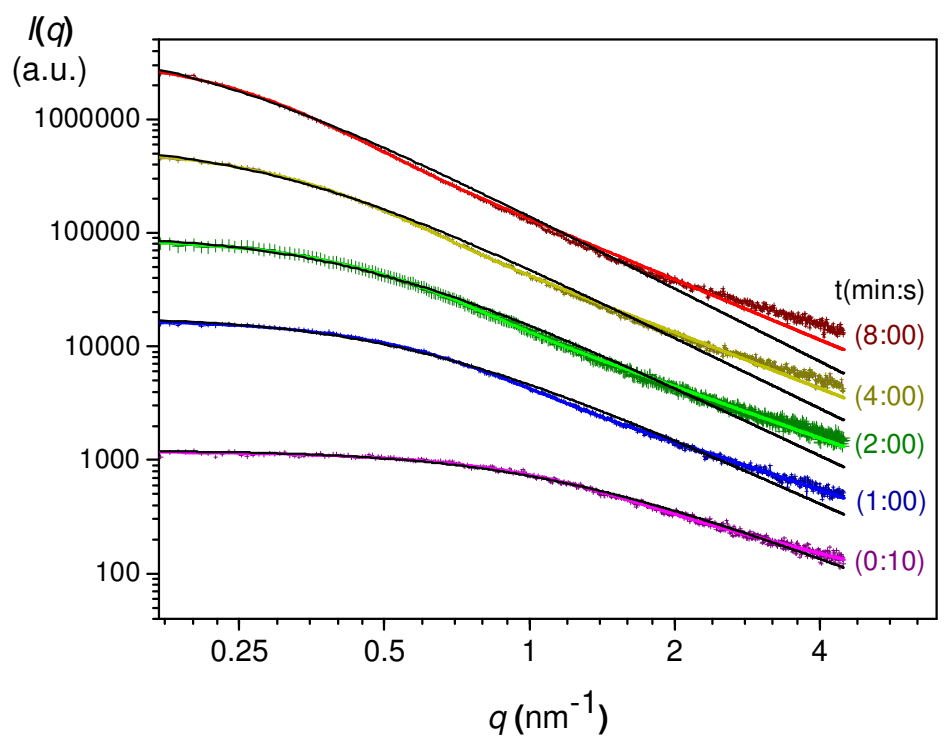

Figure 3.3. Fitted experimental data of sample $\mathrm{NbT} 25$ at different time intervals by using either the exponential cutoff model (black lines) or the Schultz cutoff model (colored lines).

Immediately after mixing the niobium pentaethoxide solutions and diluted nitric acid solutions in the absence of BTESE (sample NbT25 and NbT40), small agglomerates with $R_{\mathrm{G}}>1 \mathrm{~nm}$ were formed instantaneously (Figure 3.4a). Then, the agglomerates grew further, roughly following $R_{\mathrm{G}} \sim t^{0.5}$. Since the initial stage of the growth process was described with a growth exponent $<1$, this stage cannot be described by a gelling agglomeration kernel, despite the fact that these systems will gel eventually. Instead, it could be described well by Equation (3.23). The optimized fit parameters are given in Table 3.1.

The ratio $I_{0} / V_{\mathrm{A}}$ (Figure $3.4 \mathrm{~b}$ ), which is proportional to the mass of agglomerates in the sol, was used as a measure of the extent of particle 
nucleation. At both temperatures roughly $50 \%$ already nucleated before the first SAXS data points were recorded. After $t=2 \mathrm{~min}, I_{0} / V_{\mathrm{A}}$ leveled off. Thus, the mass concentration of agglomerates became nearly constant after $t=2$ min. Therefore, all growth after $t=2$ min occurred predominantly by cluster-cluster aggregation.

A similar trend was observed for the mass-fractal dimension $D_{\mathrm{f}}$. In the initial stage of the reaction a substantial number of primary particles nucleated. However, the extent of agglomeration of these primary particles was limited. Therefore, the number of primary particles that built up the agglomerate was too small for the agglomerate to be regarded as a mass fractal. While the agglomerates grew in time, an increasing number of primary particles contributed to the agglomerate until eventually welldefined mass-fractals were formed. In the case of sample NbT25, at $t=$ $8 \mathrm{~min}$ the fractal region ranged over approximately one order of magnitude in a range between $0.4 \mathrm{~nm}^{-1}<q<4 \mathrm{~nm}^{-1}$ (Figure 3.3) and a mass-fractal dimension could be roughly established. Beyond this point the value of $D_{f}$ levelled off towards $D_{\mathrm{f}}=1.88$ (Table 3.1), as illustrated in Figure 3.4c. At $T=$ $40^{\circ} \mathrm{C}$ the particles grew substantially faster, the rate constant $k$ (Table 3.1) was roughly 8 times larger than at $T=25^{\circ} \mathrm{C}$, while the growth exponent $p$ was roughly 1.2 times smaller. Effectively, the agglomerates grew 4 times faster at $T=40^{\circ} \mathrm{C} . R_{\mathrm{G}}$ reached $\sim 6 \mathrm{~nm}$ after $t=2 \mathrm{~min}$, as compared to $T=25^{\circ} \mathrm{C}$ where a similar gyration radius was reached after $t=8 \mathrm{~min}$. Consequently, at $T=40^{\circ} \mathrm{C} D_{\mathrm{f}}$ levelled off to $D_{\mathrm{f}}=1.91$ already after $2 \mathrm{~min}$ of reaction time.

At $T=25^{\circ} \mathrm{C}$ a decreasing value of $Z$ and an increasing value of $C \cdot C_{P}$ within the first $8 \mathrm{~min}$ imply an increasing polydispersity within this time frame. After $t=8 \mathrm{~min}$ both values levelled off. This trend can be explained by the extent of nucleation as characterized by the ratio $I_{0} / V_{\mathrm{A}}$. As long as new primary particles nucleate while other agglomerates grow the polydispersity will continue to increase. However, as the ratio $I_{0} / V_{\mathrm{p}}$ levelled off, $Z$ and $C \cdot C_{p}$ levelled off as well. This is consistent with agglomeration kernels that imply a self-preserving particle distribution during growth of fractalic agglomerates $[38,47,49,51-53]$. For sake of clarity the trends of $Z$ and $C \cdot C_{P}$ at $T=40^{\circ} \mathrm{C}$ are omitted since these trends strongly overlap with the data at $T=25^{\circ} \mathrm{C}$. Nevertheless, throughout the reaction process their values remained roughly constant within the error margins of the fit. Optimized values are listed in Table 3.1. 
At both temperatures the agglomeration process was DLCA as indicated by final $D_{\mathrm{f}} \sim 1.9$ and $C \cdot C_{\mathrm{P}} \sim 1.0$ values. The growth exponent $p$ was consistent with $\lambda$ close to zero, which confirms the proposed DLCA mechanism. Yet, the $C_{p}$ value calculated from the $\lambda$ was $\sim 1.5$, larger than the static $C_{p}$ values of $\sim 1.0$ as derived from $Z$. Possibly, since $C$ is related to the geometry of a single agglomerate, it is lower in the present case $\left(C^{2} / 3\right)$ than the previously reported experimental results implied [38]. Our findings suggest a lower variation in branch sizes per agglomerate, i.e. less elongated and more spherically shaped agglomerates.
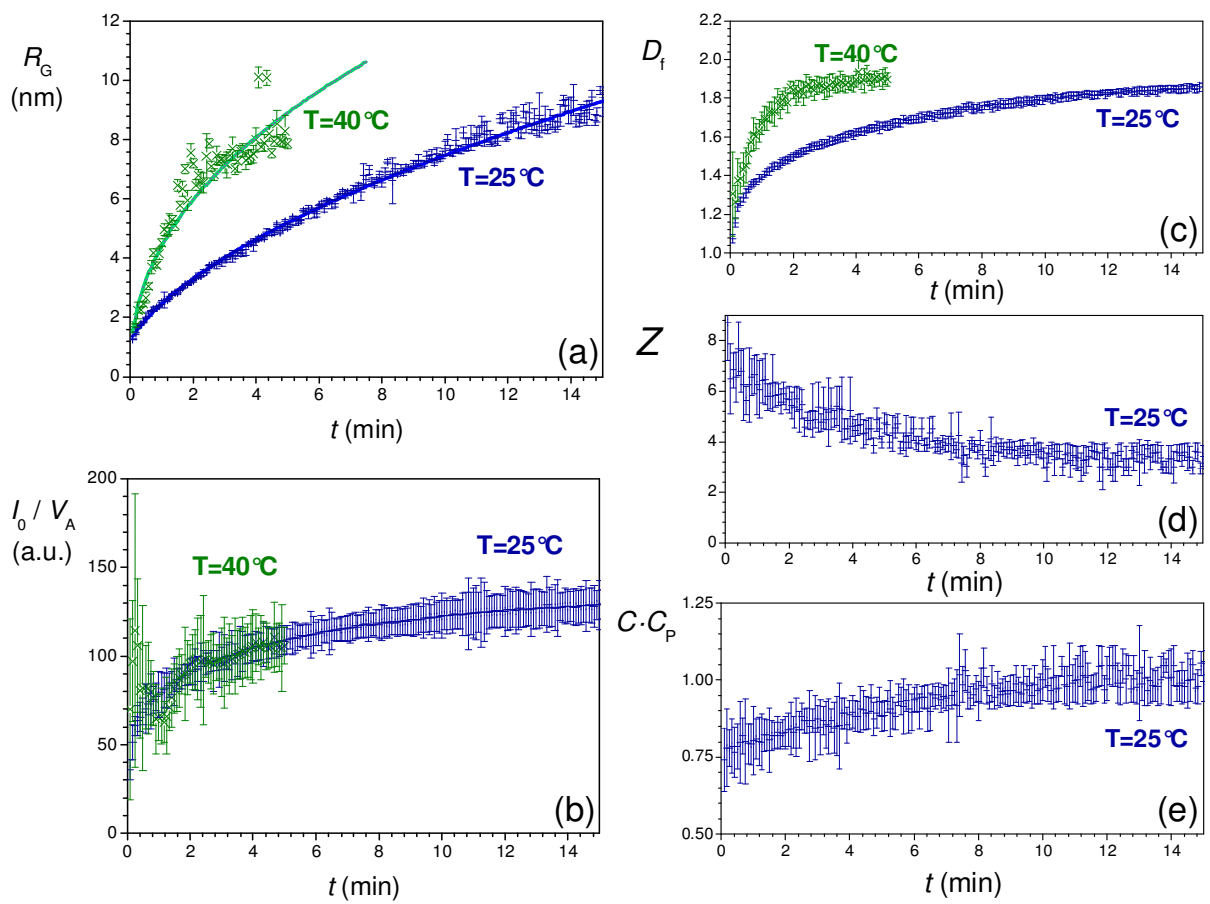

Figure 3.4. Optimized parameters derived from fitting the Schultz cutoff function for mixing experiments without BTESE. a) $R_{\mathrm{G}}$, b) $I_{0} / V_{\mathrm{A}}$, and c) $D_{\mathrm{f}}$ of NbT25 and $\mathrm{NbT} 40$;d) $Z$ and e) $C \cdot C_{\mathrm{p}}$ of sample NbT25. 
Table 3.1 Characteristic parameters of the self preserving Smoluchovski-type reaction kernels of fractalic particles with a Schultz cutoff function.

\begin{tabular}{|l|lll|lll|lll|lll|lll|}
\hline Sample & \multicolumn{3}{|l|}{ NbT25 } & \multicolumn{3}{l|}{ NbT40 } & & \multicolumn{3}{l|}{ SiNbT25 } & & \multicolumn{2}{l|}{ SiNbT40 } \\
\hline$R_{\mathrm{Gt}=0}$ & 1.22 & \pm & 0.02 & 0 & & & 1.66 & \pm & 0.04 & 1.92 & \pm & 0.01 \\
$k\left(\mathrm{~min}^{-1}\right)$ & 3.61 & \pm & 0.11 & 30 & \pm & 6 & 3.6 & \pm & 0.60 & 2.7 & \pm & 0.5 \\
$p$ & 0.56 & \pm & 0.01 & 0.44 & \pm & 0.02 & 0.14 & \pm & 0.02 & 0.18 & \pm & 0.02 \\
\hline$D_{\text {ffinal }}$ & 1.88 & \pm & 0.01 & 1.91 & \pm & 0.04 & 1.68 & \pm & 0.01 & 1.66 & \pm & 0.02 \\
$Z_{\text {final }}$ & 3.2 & \pm & 0.6 & 4.8 & \pm & 1.6 & 2.58 & \pm & 0.13 & 2.84 & \pm & 0.03 \\
$C \cdot C_{\text {Pfin }}\left(Z, D_{\mathrm{f}}\right)$ & 1.0 & \pm & 0.1 & 0.9 & \pm & 0.2 & 1.1 & \pm & 0.1 & 1.0 & \pm & 0.1 \\
\hline$\lambda$ & 0.04 & \pm & 0.01 & -0.20 & \pm & 0.05 & -3.2 & \pm & 0.1 & -2.4 & \pm & 0.1 \\
$C_{\mathrm{P}}\left(\lambda, D_{\mathrm{f}}\right)$ & 1.5 & \pm & 0.1 & 1.5 & \pm & 0.1 & & & & & & \\
\hline
\end{tabular}

The agglomeration of sol-gel mixtures that contained both BTESE and NPE (samples SiNbT25 and SiNb40) were characterized by the parameters $Z, R_{G}$ and the ratio $I_{0} / V_{\mathrm{A}}$ (Figure 3.5). After a very short induction period, the $Z$ parameter remained constant at $Z^{\sim} 2.5$ at both temperatures $\left(25^{\circ} \mathrm{C}\right.$ and $40^{\circ} \mathrm{C}$ ), see Figure $3.5 a, b$ (colored symbols) except for several frames where $Z \sim 2$ (grey symbols in Figure 3.5a,b). Especially in the early stages of the reaction, when the size of the fractal regime is very limited, deviations of the Z-parameter were observed. Moreover, due to the relatively short measurements it may be sensitive to thermal noise as well. Despite some deviations, the $Z$-values were clearly larger than zero. Thus, these systems were more monodisperse as implied by the exponential cutoff model.

The deviation in $Z$ led to systematically overestimated values of $R_{G}$ and $I_{0} / V_{\mathrm{A}}$, see grey data points in Figure 3.5c-e. However, despite this deviation, these data points reveal the same trend in $R_{\mathrm{G}}$ and $I_{0} / V_{\mathrm{A}} \cdot R_{\mathrm{G}}$ increased most rapidly in the first 10s after mixing, before the first SAXS profiles were recorded. After the first $10 \mathrm{~s} R_{\mathrm{G}}$ grew slowly further by roughly $25 \%$ at both temperatures. By comparing data from the experiments with and without BTESE, it can be concluded that the presence of BTESE had a negligible effect on the initial size of nucleated particles. However, subsequent growth of niobia clusters was clearly suppressed by the presence of BTESE. The growth exponent $p$ was substantially smaller, which led to unrealistic values of $\lambda$ (Table 3.1) when it is assumed that agglomeration can be described by irreversible Smoluchowski type rate equations. Instead, the 
reduced growth exponents $p$ were more consistent with reversible LifshitzSlyozov type agglomeration kernels [48, 54-56]. Most likely, the nucleated niobia particles/clusters were surrounded by BTESE derived moieties, which affected their agglomeration behaviour.

In analogy with the experiments without BTESE, nucleation occurred predominantly within the first $2 \mathrm{~min}$ as indicated by the ratio $I_{0} / V_{\mathrm{A}}$ (Figure $3.5 \mathrm{~d}, \mathrm{e})$. However, the values of $I_{0} / V_{\mathrm{A}}$ were substantially larger, which therefore included the nucleation from both NPE and BTESE precursors. Within a short nucleation period $Z$ levelled off (Figure 3.5a,b) even more quickly than $I_{0} / V_{\mathrm{A}}$. The subsequent growth process did not affect polydispersity. Compared to the experiments without BTESE both $Z$ and $D_{f}$ levelled off at lower values, which effectively resulted in a similar value for $C \cdot C_{P}$.
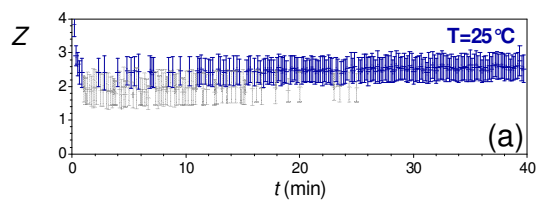

Z
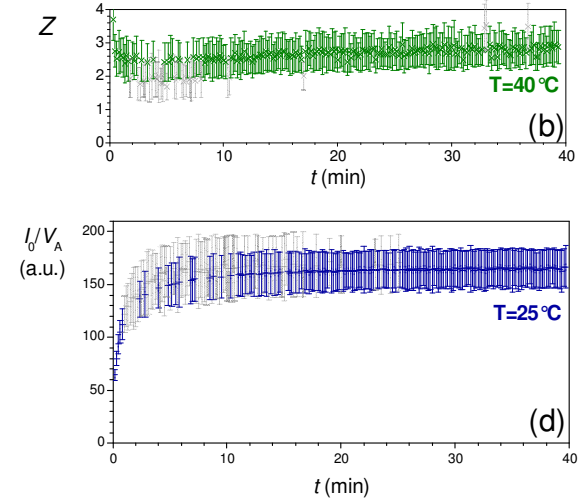
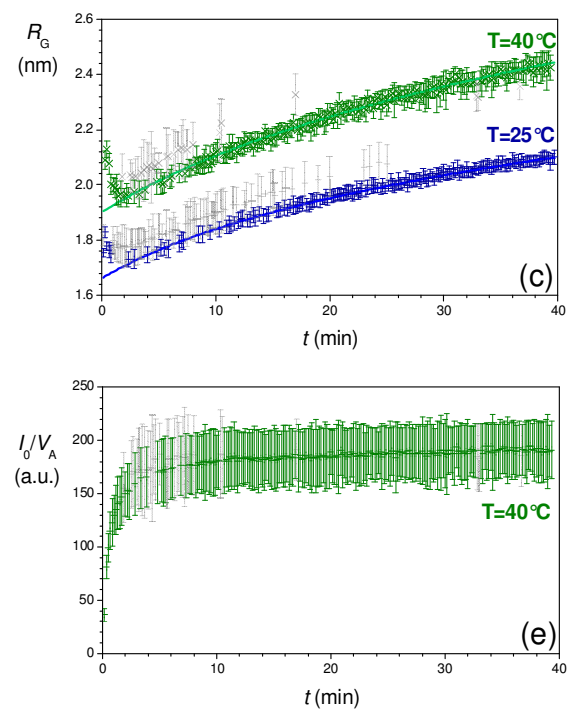

Figure 3.5. Optimized parameters derived from fitting the Schultz cutoff function for mixing experiments with BTESE. a) $Z$ of SiNbT25, b) $Z$ of SiNbT40, c) $R_{G}$ of SiNbT25 and SiNbT40, d) $I_{0} / V_{\mathrm{A}}$ of SiNbT25 and e) $I_{0} / V_{\mathrm{A}}$ of SiNbT40. 


\subsection{Conclusions}

After a short nucleation period of $\sim 2$ min, growth of NPE-derived clusters occurred through diffusion limited cluster aggregation following irreversible Smoluchowski type agglomeration kernels. The DLCA type mechanism led to relatively sharp cutoff functions that were much better described by the Schultz cutoff model than by the exponential cutoff model. The derived $C \cdot C_{P}$ values levelled off at $1.0 \pm 0.1$, being consistent with DLCA type selfpreserving agglomeration kernels.

In the sols with BTESE, the speed of nucleation was scarcely affected by the presence of BTESE. Nevertheless, further growth of the sols was clearly affected, as expressed by a reduced value of both the fractal dimension $D_{\mathrm{f}}$ and growth exponent $p$ after a certain time of reaction. The reduced growth exponent could not be described by irreversible Smoluchowski type kernels and was more consistent with reversible Lifshitz-Slyozov type agglomeration kernels. The growth of the niobia clusters was clearly suppressed by the presence of BTESE.

\subsection{References}

1. Oliver, M.S., et al., Molecular Origins of the Mechanical Behavior of Hybrid Glasses. Adv. Funct. Mater., 2010. 20(17): p. 2884-2892.

2. Castricum, H.L., et al., Hydrothermally stable molecular separation membranes from organically linked silica. J. Mater. Chem., 2008. 18(18): p. 2150-2158.

3. Castricum, H.L., et al., High-performance hybrid pervaporation membranes with superior hydrothermal and acid stability. J. Membr. Sci., 2008. 324(1-2): p. 111-118.

4. Kanezashi, M., et al., Organic-inorganic hybrid silica membranes with controlled silica network size: Preparation and gas permeation characteristics. J. Membr. Sci., 2010. 348(1-2): p. 310-318.

5. Agirre, I., et al., Hybrid organosilica membranes and processes: Status and outlook. Sep. Purif. Technol., 2014. 121: p. 2-12.

6. Agirre, I., et al., Acetalization reaction of ethanol with butyraldehyde coupled with pervaporation. Semi-batch 
pervaporation studies and resistance of $\mathrm{HybSi}<$ sup $>{ }^{\circledR}</$ sup $>$ membranes to catalyst impacts. J. Membr. Sci., 2011. 371(1): p. 179-188.

7. Kreiter, R., et al., Evaluation of hybrid silica sols for stable microporous membranes using high-throughput screening. J. SolGel Sci. Technol., 2010: p. 1-8.

8. Igi, R., et al., Characterization of co-doped silica for improved hydrothermal stability and application to hydrogen separation membranes at high temperatures. J. Am. Ceram. Soc., 2008. 91(9): p. 2975-2981.

9. Kanezashi, M. and M. Asaeda, Hydrogen permeation characteristics and stability of Ni-doped silica membranes in steam at high temperature. J. Membr. Sci., 2006. 271(1-2): p. 86-93.

10. Qi, H., et al., Effect of $\mathrm{Nb}$ content on hydrothermal stability of a novel ethylene-bridged silsesquioxane molecular sieving membrane for H2/CO2 separation. J. Membr. Sci., 2012. 421-422(0): p. 190200.

11. Qi, H., et al., Hybrid Organic-Inorganic Microporous Membranes with High Hydrothermal Stability for the Separation of Carbon Dioxide. ChemSusChem, 2010. 3(12): p. 1375-1378.

12. Boffa, V., et al., Microporous niobia-silica membrane with very low CO2 permeability. ChemSusChem, 2008. 1(5): p. 437-443.

13. Boffa, V., et al., Microporous niobia-silica membranes: Influence of sol composition and structure on gas transport properties. Micropor. Mesopor. Mat., 2009. 118(1-3): p. 202-209.

14. Yoshida, K., et al., Hydrothermal stability and performance of silicazirconia membranes for hydrogen separation in hydrothermal conditions. J. Chem. Eng. Jpn, 2001. 34(4): p. 523-530.

15. Livage, J., M. Henry, and C. Sanchez, Sol-gel chemistry of transition metal oxides. Prog. Solid State Ch., 1988. 18(4): p. 259-341.

16. Kessler, V.G., et al., New insight in the role of modifying ligands in the sol-gel processing of metal alkoxide precursors: A possibility to approach new classes of materials. J. Sol-Gel Sci. Technol., 2006. 40(2-3): p. 163-179.

17. Brinker, C.J.S., G. W. , Sol Gel Science, The Physics and Chemistry of Sol-Gel Processing. Vol. 1. 1990, San Diego: Acedemic Press. 1-881.

18. Sanchez, J. and A. McCormick, Kinetic and thermodynamic study of the hydrolysis of silicon alkoxides in acidic alcohol solutions. The J. Phys. Chem., 1992. 96(22): p. 8973-8979. 
19. Rankin, S.E. and A.V. McCormick, Hydrolysis pseudoequilibrium: challenges and opportunities to sol-gel silicate kinetics. Chem. Eng. Sci., 2000. 55(11): p. 1955-1967.

20. Assink, R.A. and B.D. Kay, The chemical kinetics of silicate sol-gels: Functional group kinetics of tetraethoxysilane. Colloids and Surfaces A: Physicochemical and Engineering Aspects, 1993. 74(1): p. 1-5.

21. Pouxviel, J., et al., NMR study of the sol/gel polymerization. J. NonCryst. Solids, 1987. 89(3): p. 345-360.

22. Bonhomme, C., et al., Advanced Solid State NMR Techniques for the Characterization of Sol-Gel-Derived Materials. Acc. Chem. Res., 2007. 40(9): p. 738-746.

23. Harris, M.T., et al., FTIR spectroscopy, SAXS and electrical conductivity studies of the hydrolysis and condensation of zirconium and titanium alkoxides. J. Sol-Gel Sci. Technol., 1997. 8(1-3): p. 4147.

24. Simonsen, M.E. and E.G. Søgaard, Sol-gel reactions of titanium alkoxides and water: influence of $\mathrm{pH}$ and alkoxy group on cluster formation and properties of the resulting products. J. Sol-Gel Sci. Technol., 2010. 53(3): p. 485-497.

25. Broussous, L., et al., SAXS Study of Formation and Growth of Tin Oxide Nanoparticles in the Presence of Complexing Ligands. J Phys. Chem. B, 2002. 106(11): p. 2855-2860.

26. Hu, M.-C., et al., Probing the early-stage/rapid processes in hydrolysis and condensation of metal alkoxides. Journal of materials science, 2000. 35(8): p. 1957-1971.

27. Wilkinson, A.P., et al., In situ $x$-ray diffraction study of crystallization kinetics in PbZr1-xTixO3,(PZT, $x=0.0,0.55,1.0)$. Chem. Mater., 1994. 6(6): p. 750-754.

28. Fan, J., S.W. Boettcher, and G.D. Stucky, Nanoparticle assembly of ordered multicomponent mesostructured metal oxides via a versatile sol-gel process. Chem. Mater., 2006. 18(26): p. 6391-6396.

29. Percy, M., et al., The influence of 6 -diketones on the induction times for hydrolysis of zirconium (IV) alkoxides. J. Mater. Chem. 1999. 9(2): p. 499-505.

30. Sedlar, M. and M. Sayer, Reactivity of titanium isopropoxide, zirconium propoxide and niobium ethoxide in the system of 2methoxyethanol, 2,4-pentanedione and water. J. Sol-Gel Sci. Technol., 1995. 5(1): p. 27-40.

31. Ranjit, K.T. and K.J. Klabunde, Solvent effects in the hydrolysis of magnesium methoxide, and the production of nanocrystalline 
magnesium hydroxide. An aid in understanding the formation of porous inorganic materials. Chem. Mater., 2005. 17(1): p. 65-73.

32. Tanabe, K., Application of niobium oxides as catalysts. Catal. Today, 1990. 8(1): p. 1-11.

33. Francisco, M.S.P., R. Landers, and Y. Gushikem, Local order structure and surface acidity properties of $a \mathrm{Nb}<$ sub $>2</$ sub $>0<$ sub $>5</$ sub $>/$ SiO $<$ sub $>2</$ sub $>$ mixed oxide prepared by the solgel processing method. J. Solid State Ch., 2004. 177(7): p. 24322439.

34. Yoshida, H., et al., Control of the structure of niobium oxide species on silica by the equilibrium adsorption method. Catal. Today, 1996. 28(1): p. 79-89.

35. Bras, W., et al., Recent experiments on a combined smallangle/wide-angle $X$-ray scattering beam line at the ESRF. J. Appl. Crystallogr., 2003. 36(3 I): p. 791-794.

36. Bras, W., et al., Field-induced alignment of a smectic-a phase: $A$ time-resolved $x$-ray investigation. J. Chem. Phys., 2004. 121(9): p. 4397-4413.

37. Besselink, R., et al., Evolution of microstructure in mixed niobiahybrid silica thin films from sol-gel precursors. J. Colloid Interf. Sci., 2013. 404(0): p. 24-35.

38. Sorensen, C.M. and G.M. Wang, Size distribution effect on the power law regime of the structure factor of fractal aggregates. Phys. Rev. E, 1999. 60(6 B): p. 7143-7148.

39. Teixeira, J., Small-angle scattering by fractal systems. J. Appl. Crystallogr., 1988. 21(6): p. 781-785.

40. Chen, S.H. and J. Teixeira, Structure and fractal dimension of protein-detergent complexes. Phys. Rev. Lett., 1986. 57(20): p. 2583-2586.

41. Feigin, L.A. and D.U. Svergun, Struncture analysis by small angle $X$ ray and neutron scattering1987, New York: Plenum Press. 334.

42. Porod, G., Small Angle X-ray Scattering. Vol. 15. 1982, New York: Acedamic Press. 515.

43. Kotlarchyk, M. and S.H. Chen, Analysis of small angle neutron scattering spectra from polydisperse interacting colloids. J. Chem. Phys., 1983. 79: p. 2461.

44. Stawski, T.M., et al., Erratum: Nanoscale structure evolution in alkoxide-carboxylate sol-gel precursor solutions of barium titanate (J. Phys. Chem. C (2010) 115 (20449-20459) DOI: 10.1021/jp206572q). J. Phys. Chem. C, 2011. 115(48): p. 24028. 
45. Stawski, T.M., et al., Nanoscale structure evolution in alkoxidecarboxylate sol-gel precursor solutions of barium titanate. J. Phys. Chem. C, 2011. 115(42): p. 20449-20459.

46. Pontoni, D., T. Narayanan, and A.R. Rennie, Nucleation and growth kinetics of colloidal silica, 2004. p. 227-230.

47. Oh, C. and C.M. Sorensen, Light scattering study of fractal cluster aggregation near the free molecular regime. J. Aerosol Sci., 1997. 28(6): p. 937-957.

48. Pontoni, D., T. Narayanan, and A. Rennie, Time-resolved SAXS study of nucleation and growth of silica colloids. Langmuir, 2002. 18(1): $p$. 56-59.

49. Pierce, F., C. Sorensen, and A. Chakrabarti, Computer simulation of diffusion-limited cluster-cluster aggregation with an Epstein drag force. Physical Review E, 2006. 74(2): p. 021411.

50. Ball, R., et al., Universal kinetics in reaction-limited aggregation. Phys. Rev. Lett., 1987. 58(3): p. 274.

51. Wu, M.K. and S.K. Friedlander, Enhanced power law agglomerate growth in the free molecule regime. J. Aerosol Sci., 1993. 24(3): p. 273-282.

52. Van Dongen, P. and M. Ernst, Dynamic scaling in the kinetics of clustering. Phys. Rev. Lett., 1985. 54(13): p. 1396.

53. Dekkers, P.J. and S.K. Friedlander, The self-preserving size distribution theory: I. Effects of the Knudsen number on aerosol agglomerate growth. J. Colloid Interf. Sci., 2002. 248(2): p. 295-305.

54. Lifshitz, I.M. and V.V. Slyozov, The kinetics of precipitation from supersaturated solid solutions. J. Phys. Chem. Solids, 1961. 19(1): p. 35-50.

55. Conti, M., A. Lipshtat, and B. Meerson, Scaling anomalies in the coarsening dynamics of fractal viscous fingering patterns. Phys. Rev. E, 2004. 69(3): p. 031406.

56. Sharon, E., et al., Coarsening of Fractal Viscous Fingering Patterns. Phys. Rev. Lett., 2003. 91(20): p. 205504. 
Evolution of microstructure in mixed niobia-hybrid silica thin films from solgel precursors 


\subsection{Abstract}

The evolution of structure in sol-gel derived mixed bridged silsesquioxaneniobium alkoxide sols and drying thin films was monitored in-situ by small angle X-ray scattering. Since sol-gel condensation of metal alkoxides proceeds much faster than that of silicon alkoxides, the incorporation of $d$ block metal dopants into silica typically leads to formation of densely packed nano-sized metal oxide clusters that we refer as metal oxide building blocks in a silica-based matrix. SAXS was used to study the process of niobia building block formation while drying the sol as a thin film at 40$80^{\circ} \mathrm{C}$. The SAXS curves of mixed niobia-hybrid silica sols were dominated by the electron density contrast between sol particles and surrounding solvent. As the solvent evaporated and the sol particles approached each other, a correlation peak emerged. Since TEM microscopy revealed the absence of mesopores, the correlation peak was caused by a heterogeneous system of electron-rich regions and electron poor regions. The regions were assigned to the presence of small clusters that are rich in niobium which are dispersed in a matrix that mainly consisted of hybrid silica. The correlation peak corresponded with correlation distances in the range between 1 and $3 \mathrm{~nm}$ that was associated with the typical distances between the electron dense clusters. A relationship between the prehydrolysis time of the silica precursor and the size of the niobia building blocks was observed. When 1,2-bis(triethoxysilyl)ethane was first hydrolyzed for $30 \mathrm{~min}$ before adding niobium pentaethoxide, the niobia building blocks reached a radius of $0.4 \mathrm{~nm}$. Simultaneous hydrolysis of the two precursors resulted in somewhat larger average building block radii of 0.5 to $0.6 \mathrm{~nm}$. This study shows that acid catalyzed sol-gel polymerization of mixed hybrid silica niobium alkoxides can be rationalized and optimized by monitoring the structural evolution using time-resolved SAXS.

\subsection{Introduction}

Molecular separation with membranes is an attractive alternative for distillation since it can lead to substantial energy savings in the dehydration of biomass fuels [1] and organic solvents [2-3]. Hybrid organosilica membranes are very suitable for such tasks, since these materials combine 
the advantages of both organic and inorganic membranes, i.e.: the high fracture resistance and hydrothermal stability of organic membranes and the high flux and thermal stability of inorganic membranes [4-5]. Exposure of silica to moisture at $70^{\circ} \mathrm{C}$ already leads to hydrolysis of Si-O-Si bonds. The presence of organic bridges in the network made the network less susceptible for hydrolysis. However, further improvement is desirable for industrial application.

A possible approach to improve the hydrothermal and chemical stability of siloxanes and silsesquioxanes is by doping transition metals into the silicon oxide network, such as titanium [6], zirconium [7-8] niobium [9], nickel [10] or cobalt [11]. However, sol-gel synthesis of transition metal-doped siloxanes usually leads to the formation of dense nanosized transition metal oxide building blocks embedded inside an amorphous silica matrix [12], as it was observed by transmission electron microscopy (TEM) or small angle $X-$ ray scattering (SAXS) [13-14]. Transition metal centers easily form cationic complexes that are surrounded by a large number of ligands (coordination number 6-8), in contrast to silicon which commonly forms neutral tetragonal complexes [15]. Moreover, the metal alkoxide bonds are more labile and susceptible towards hydrolysis and condensation compared to the more covalently bonded tetragonal silicon alkoxide bonds [16]. Several strategies have been applied to improve the homogeneity of mixed transition metal/silicon oxide materials, but the intermixing on atomic scale, which is crucial for sufficient membrane permeability, is still a challenge.

Our interest is particularly focused on the atomic mixing of niobium in a hybrid silica matrix. ${ }^{17} \mathrm{O}$ MAS NMR experiments revealed that acid-catalyzed sol-gel synthesis leads to a high degree of atomic mixing at low niobium concentrations, i.e. $\mathrm{Nb} / \mathrm{Si}=14 / 86$ and 6/94 [17]. Even if amorphous niobia building blocks were formed, their crystallization would only occur at temperatures above $400^{\circ} \mathrm{C}$ [18]. which is higher than the annealing temperature of $300^{\circ} \mathrm{C}$ of hybrid organosilica membranes [19]. No limitations are thus expected with regard to matrix integrity. Hydrated $\mathrm{Nb}_{2} \mathrm{O}_{5}$ forms strong Lewis and Bronsted acid sites upon annealing at $100^{\circ} \mathrm{C}$ at $\mathrm{pH}<5.6$ (indicator method) [20]. Substitution in a silica matrix was found to enhance the Lewis acidity of the $\mathrm{Nb}$ centers as compared to that in $\mathrm{Nb}_{2} \mathrm{O}_{5}$ [21]. Contrary to vanadium (V) oxides, which are frequently used as acid catalysts, niobium oxides are more difficult to reduce and chemically more stable [22]. It is therefore a promising catalyst for application at 
moderate temperatures, in e.g.: preferential $\mathrm{CO}$ oxidation in hydrogen-rich environments [23], esterification [24-25], dehydrogenation [26], aldol condensation [27] and conversion of the biomass derivative $\gamma$ valerolacetone into pentanoic acid [28]. The presence of acid sites was found to enhance chemisorption of $\mathrm{CO}_{2}$ on mixed $\mathrm{SiO}_{2}-\mathrm{Nb}_{2} \mathrm{O}_{5}$ and hybrid silica $\mathrm{Nb}_{2} \mathrm{O}_{5}$ microporous membranes and therefore strongly reduced the $\mathrm{CO}_{2}$ permeability in comparison with nonpolar gases like $\mathrm{H}_{2}, \mathrm{CH}_{4}$ and $\mathrm{SF}_{6}$ [29-30].

The final microstructure is established after a sol has been coated and dried on a support. The details of the drying processes of sol-gel films are often not well understood. In situ small X-ray scattering has been found to be a valuable method for monitoring the reorganization of meso/microstructure in drying systems, for instance in the self-assembly of mesostructured solgel films [31] and the formation of different electron-dense/lean phases in barium titanate films from alkoxide-carboxylate systems [32]. We used time-resolved SAXS to investigate the reorganization of the microstructure and the formation of different electron dense/lean phases during the drying process of thin sol-gel films of niobium pentaethoxide and 1,2bis(triethoxysilyl)ethane.

\subsection{Experimental Section}

\subsubsection{Synthesis of mixed niobia/BTESE sols}

All precursor solutions were prepared inside a glovebox under nitrogen atmosphere. The reflux synthesis was performed under atmospheric conditions. A $0.45 \mathrm{~mol} / \mathrm{L}$ 1,2-bis-triethoxysilyl-ethane (BTESE) sol was prepared by adding dropwise an acidic ethanol solution [33] to a $0.9 \mathrm{~mol} / \mathrm{L}$ solution of BTESE (ABCR chemicals, 97\%) in dry ethanol with a hydrolysis ratio $\left[\mathrm{H}_{2} \mathrm{O}\right] /[(\mathrm{Si})-\mathrm{OEt}]=1$ and an acid ratio $\left[\mathrm{HNO}_{3}\right] /[(\mathrm{Si})-\mathrm{OEt}]=1 / 30$. In a separate bottle niobium pentaethoxide (NPE, ABCR chemicals, 99.99\%) was diluted in ethanol yielding a molar concentration $[N P E]=0.54 \mathrm{~mol} / \mathrm{L}$. The BTESE sols were all refluxed at $\mathrm{T}=60^{\circ} \mathrm{C}$ for $60 \mathrm{~min}$. The NPE solution was added after either 0,30 or 60 minutes of refluxing, and these samples are designated as p00, p30 and p60, respectively. Hence, the NPE solution was refluxed at $\mathrm{T}=60^{\circ} \mathrm{C}$ for 60,30 or $0 \mathrm{~min}$, respectively. The molar ratio 
[Nb]/[Si] was kept constant at $1 / 4$ in this series and the overall initial ethoxide concentration was kept constant at [OEt] $=2.7 \mathrm{~mol} / \mathrm{L}$. The samples were dried as thin films using a drying setup with a rotating aluminum rotor-hear that was covered with Kapton foil as described in more detail elsewhere [32]. All samples were heated using a $150 \mathrm{~W}$ infrared lamp. Samples p00 and p60 were dried at $T=60^{\circ} \mathrm{C}$ and samples $\mathrm{p} 30$ were dried at $T=40,60$ or $80^{\circ} \mathrm{C}$, respectively.

\subsubsection{SAXS Experiments}

The colloidal samples were placed in capillary glass tubes (Hilgenberg, Germany) of $80 \mathrm{~mm}$ length, $1.5 \mathrm{~mm}$ diameter and $\sim 10 \mu \mathrm{m}$ wall thickness. Small-angle X-ray scattering was carried out using synchrotron radiation on the Dutch-Belgian beamline, DUBBLE BM-26B of the ESRF in Grenoble [34]. The $X$-ray beam with an energy of $16 \mathrm{keV}$ was focused on a corner of the $2 \mathrm{D}$ Pilatus $1 \mathrm{M}$ CCD-detector to maximize the covered range of scattering angles. A beam stop was applied to shield the detector from the direct beam and avoid saturation of the outgoing signal. The detector was placed at a distance of $1.5 \mathrm{~m}$ from the sample, which allowed us to obtain data in the range $0.20<q<9.00 \mathrm{~nm}^{-1}$. All scattering data were found to be independent of the scattering angle in the plane of the detector. As this indicates that the samples were isotropically dispersed, the measured intensities from all channels with the same $q$ value were averaged. Silver behenate was used as calibration standard for the determination of the absolute scale of the scattering vector $q$ in our experiments [35].

The scattering intensity of the capillary containing a given colloidal sample was collected as a function of the scattering vector $q$. All curves were normalized by dividing the scattering intensity by both the time of data acquisition and the total intensity of the scattered signal. A background subtraction procedure was carried out. For sols, the scattering signal of a capillary filled with ethanol was subtracted, while for the drying sols only the scattering signal of the clean kapton foil was subtracted. The static SAXS data of sols were obtained in 5 min data collection periods and the in situmeasurements in 1 min periods. 


\subsubsection{TEM measurements}

From sample p60, 30 $\mu \mathrm{L}$ sol was spin-cast (Laurell WS- 400B-6NPP-Lite spincoater) onto holey carbon TEM copper grids (CF200-Cu, Electron Microscopy Sciences) at $4000 \mathrm{rpm}$ for $40 \mathrm{~s}$. Then the as-prepared films were dried at $60^{\circ} \mathrm{C}$ for 24 hours in a furnace and used for transmission electron microscopy characterization (Philips CM300STFEG at 300 kV). Samples were investigated at low magnification to find typical areas and features of interest were examined at high magnification (GATAN 20482048 Ultrascan 1000 CCD camera). The microscope software packages GATAN Microscopy Suite 1.8 .

\subsection{Derivation of the Scattering intensity function.}

Positive interference of scattered waves originates either from electrons within the same particle (intraparticle interference) or from electrons located in different particles (interparticle correlations). The scattering intensity can therefore be divided into a form factor, $P(q)$ and a structure factor $S(q)$, scaled with the number density of particles, $N$, and the contrast in electron density between particles and their surrounding medium, $\Delta \rho$ (Equation (4.1)):

$$
I(q)=N \cdot(\Delta \rho)^{2} \cdot S(q) \cdot P(q)
$$

In the model that is described below to model the scattering of X-rays by drying sol-gel films, the sol particles are described in terms of selfassembled small primary building blocks. A building block contains a small number of niobium and/or hybrid silicon monomers and is considered as the smallest cluster of atoms that can be discriminated from the surrounding solvent in a SAXS-pattern by a difference in electron density. For the sake of simplicity, we assume that these building blocks (primary scatterers) are spherical with radius $r_{0}$. This assumption was in agreement with the majority of scattering curves that indicated a $/ \sim q^{-4}$ dependence in the high q-region. Only the initial stages of the drying process revealed a deviating behavior. These building blocks can be described by a spherical form factor $P(q)$, (4.2 [36-38]. 
$P\left(q, r_{0}\right)=\left[3 \cdot \frac{\sin \left(q \cdot r_{0}\right)-q \cdot r_{0} \cdot \cos \left(q \cdot r_{0}\right)}{q^{3} \cdot r_{0}{ }^{3}}\right]^{2}$

These building blocks may assemble into two different spatial arrangements. Firstly, they can assemble into a branched polymer-like arrangement with scattering behavior as described in section 3.1. Secondly, a condensed phase can form that gives rise to a correlation peak in the $\mathrm{X}$ ray scattering curve. In the latter case the scattering intensity can be interpreted as a liquid-like packing of building blocks as described in section 3.2. During the sol condensation and film drying process both phases may coexist. For simplicity, we assume that the two types of structures are constructed of the same type of primary scatterer, such that the total scattering intensity can be described as a linear combination of the contributions of both phases to the total scattering intensity (section 3.3).

\subsubsection{Mass-fractal-like sol particles}

Small spherical building blocks may arrange themselves randomly forming larger sol particles with a branched polymeric structure. Such assemblies of building blocks are well understood in terms of mass-fractal models [15, 3940]. Various acid-catalyzed silica sols, including $\mathrm{Nb}$-doped silica, have been modelled successfully in terms of mass fractals [41]. When monodisperse spherical building blocks of radius $r_{0}$ are packed together with a packing density that can be characterized by the so-called fractal dimension $D$, then the number of building blocks $n$ within an imaginary sphere of effective radius $r$ is described by [39-40]:

$n=\left(\frac{r}{r_{0}}\right)^{D}$

The structure function $S_{M F}$ (Equation (4.4)) of mass-fractal agglomerates was derived by Teixeira [39]. The agglomerates were giving a finite size by 
introducing a an exponentially decaying element in the autocorrelation function $(\exp (-r / \xi) / r)$, where the agglomerate size was characterized by the cut-off length $\xi$. This approach was based on the assumption the sol particle radius was distributed exponentially. Although DLCA-based systems generally have narrower size distributions [42-43], Equation (4.4) was in a good agreement with our experimental data. In addition, the expression is convenient from a practical point of view, since the Fourier transform was solved analytically.

$$
S_{M F}\left(q, D, \xi, r_{0}\right)=1+\frac{D \cdot \Gamma(D-1) \cdot \sin [(D-1) \cdot \arctan (q \cdot \xi)]}{\left(q \cdot r_{0}\right)^{D} \cdot\left(1+\frac{1}{(q \cdot \xi)^{2}}\right)^{\left(\frac{D-1}{2}\right)}}
$$

Here $\Gamma$ represents the gamma function. The radii of primary building blocks, $r_{0}$ are considered to be monodisperse. The radius of gyration of the agglomerates can be calculated from $D$ and $\xi$ via (4.5 [43]:

$$
R_{g}=\xi \cdot \sqrt{\frac{D \cdot(D+1)}{2}}
$$

\subsubsection{Packing of hard spheres}

While drying a sol, dispersed small sol particles eventually become supersaturated and assemble into larger randomly closed packed agglomerates that subsequently condense. In all mixed oxide sols these segregated assemblies gave a broad correlation peak in the corresponding scattering curves. The correlation peak indicates the presence of a modulated pattern of alternating electron rich and electron lean regions. The maximum of the correlation peak was associated with the a typical 
quasi-periodic distances between alternating electron dense regions that are separated by electron lean regions as this had been reported for phase separated systems such as ionomers [44], nanocomposits [12-13,45] and microemulsions [46]. The absence of higher order correlation peaks is due to the absence of long range order in the alternating regions. When the building blocks are considered as hard monodisperse spheres in randomly oriented liquid-like packing, the corresponding interference function is as described by Fournet [36]. They applied the equation of state from Born and Green for hard spheres ((4.6), in which $\in$ is a constant close to unity [36], $C\left(q, \phi, R_{\mathrm{S}}\right)$ the direct correlation function between spherical particles, and $R_{\mathrm{S}}$ is the separation radius of particles. The typical distance between particles equals $L_{S}=2 R_{s}$. See Figure 4.1.

$$
S_{C}\left(q, \phi, R_{S}\right)=\frac{1}{1+8 \cdot \phi \cdot \in \cdot C\left(q, \phi, R_{S}\right)}
$$

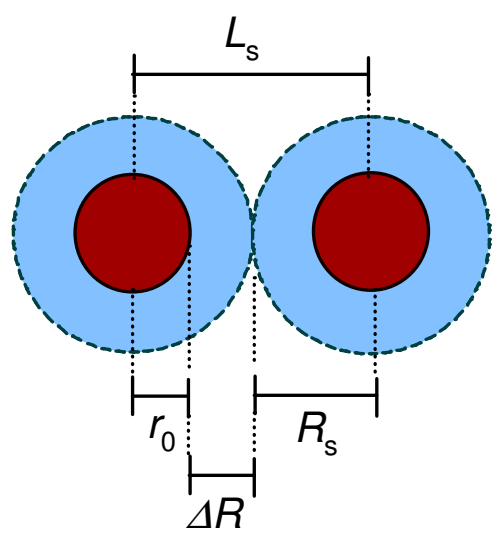

Figure 4.1. Definitions of length scales in the hard sphere model.

The parameter $\phi$ represents the local volume fraction of particles in the surroundings of a specific particle. It is defined as the ratio between the overall particle volume divided by the total available volume. It has a maximum value of 0.74 for close-packed hexagonal and cubic lattices. For 
multiple particle interactions the direct correlation function can be described by a Percus-Yevick approximation giving [44-45]:

$$
\begin{aligned}
C\left(q, \phi, L_{S}\right)= & 3 \cdot \lambda_{1} \cdot \frac{\sin (A)-A \cdot \cos (A)}{A^{3}} \\
& +3 \cdot \lambda_{2} \cdot \frac{2 \cdot A \cdot \sin (A)+\left(2-A^{2}\right) \cdot \cos (A)-2}{A^{4}} \\
& +3 / 2 \cdot \phi \cdot \lambda_{1} \cdot \frac{-A^{4} \cdot \cos (A)+4\left(3 A^{2}-6\right) \cos (A)+4\left(A^{3}-6 A\right) \cdot \sin (A)+24}{A^{6}}
\end{aligned}
$$

where

$$
A=q \cdot L_{S} \quad L_{S}=2 \cdot R_{S} \quad \lambda_{1}=\frac{(1+2 \cdot \phi)^{2}}{(1-\phi)^{4}} \quad \lambda_{2}=\frac{-6 \phi \cdot(1+\phi / 2)^{2}}{(1-\phi)^{4}}
$$

This model was used successfully by us to model the evolution of certain species upon gelation and upon drying of sol-gel precursors of $\mathrm{BaTiO}_{3}$ $[25,47]$. Nevertheless, in the experiments discussed below, the correlation peaks were broader, indicating that we need to consider a substantial variation in separation distances. Polydispersity can be introduced into this model by applying the local monodisperse approximation [48] which assumes that particles correlate only with particles of similar size. This approach reduces amount of calculation strongly, yet it still provides a reliable relationship between peak broadening and polydispersity [48]. The scattering intensity of a polydisperse correlated system, $I\left(q, \phi, \mu_{\mathrm{Ls}}, \sigma_{\mathrm{Ls}}\right)$ can thus be described by the following integral:

$$
I_{\mathrm{PC}}\left(q, \phi, \mu_{\mathrm{r}_{0}}, \sigma_{\mathrm{r}_{0}}\right)=N \cdot \Delta \rho^{2} \int P\left(q, r_{0}\right) \cdot S_{C}\left(q, \phi, R_{\mathrm{S}}\left(r_{0}\right)\right) \cdot D_{P}\left(r_{0}\right) d r_{0}
$$

Herein $P\left(q, r_{0}\right)$ is the form factor (Equation (4.2)), $S\left(q, \phi, R_{s}\left(r_{0}\right)\right)$ the structure function (Equation (4.6)) where the outer radius $R_{s}\left(r_{0}\right)$ is described as a 
function of radius $r_{0}$ of the inner electron dense region. $N\left(r_{0}\right)$ is described as the number weighted particle size distribution. However, the probability of finding correlations scales with the square of the number of electrons within the correlation sphere. Thus, when introducing either a number weighted distribution function or a volume weighted distribution, $m^{2}\left(r_{0}\right)$ or $m\left(r_{0}\right)$, respectively, needs to be introduced in the integral equation, where $m$ represents the mass of electrons within the particle radius $r_{0}$ [37]. With a uniform particle density and the particles being spherical, $m \sim r^{3}$ holds. When introducing the weighting parameter and normalizing the intensity function for introducing the weighting factor [37] the scattering intensity of polydisperse randomly packed spheres can be described by:

$$
I_{\mathrm{PC}}\left(q, \phi, \mu_{\mathrm{r}_{0}}, \sigma_{\mathrm{r}_{0}}\right)=\frac{N \cdot \Delta \rho^{2} \int P\left(q, r_{0}\right) \cdot S_{C}\left(q, \phi, R_{\mathrm{S}}\left(r_{0}\right)\right) \cdot D_{P}\left(r_{0}\right) \cdot r_{0}{ }^{p} d r_{0}}{\int D_{P}\left(r_{0}\right) \cdot r_{0}{ }^{p} d r_{0}}
$$

The parameter $p$ is equal to 6 or 3 for number and volume weighted distributions, respectively. In the case that $p=0,(4.9$ is reduced to (4.8 and the distribution can be considered as an intensity weighted distribution. Pedersen considered the outer radius that is separating the particles described by either $R_{\mathrm{S}}=C \cdot r_{0}$ or $R_{\mathrm{S}}=\Delta R+r_{0}$, where $C$ and $r_{0}$ are considered as non-distributed constants [48]. It was assumed that the variation in $R_{\mathrm{S}}$ was typically much larger than the variation in $r_{0}$. When we assume that $r_{0}$ is monodisperse, while $R_{\mathrm{S}}$ is polydisperse, the form factor can be removed from the integral. Then, the structure factor of correlated spheres with an intensity weighted polydisperse outer shell separation distance, $\left(L_{S}=2 R_{S}\right)$ can be described by:

$S_{\mathrm{PC}}\left(q, \phi, \mu_{\mathrm{Ls}}, \sigma_{\mathrm{Ls}}\right)=\int w\left(L_{\mathrm{S}}, \mu_{\mathrm{Ls}}, \sigma_{\mathrm{Ls}}\right) \cdot S_{C}\left(q, \phi, L_{\mathrm{S}}\right) \cdot d L_{\mathrm{S}}$

Here $w\left(L_{s}, \mu_{\mathrm{Ls}}, \sigma_{\mathrm{Ls}}\right)$ is a particle size distribution of average $\mu_{\mathrm{Ls}}$, with particle separation $L_{s}$ and standard deviation $\sigma_{\mathrm{Ls}}$. The parameter $p$ determines the weighting of distribution. We applied a Zimm-Schultz-Flory distribution [49] 
which gave reliable distributions to describe sol-gel derived silica particles in an earlier study [50].

$$
w\left(L_{S}, \mu_{\mathrm{Ls}}, z\right)=\frac{L_{S}{ }^{z}}{\Gamma(z+1)} \cdot\left(\frac{z+1}{\mu_{L s}}\right)^{z+1} \cdot \exp \left(-\left(\frac{z+1}{\mu_{L s}}\right) \cdot L_{S}\right)
$$

The $z$-parameter determines the broadness of the distribution and the corresponding standard deviation is given by:

$$
\sigma_{\mathrm{Ls}}=\frac{\mu_{\mathrm{L} s}}{\sqrt{z+1}}
$$

4.4.3. Heterogeneous mixture of mass-fractal-like sol particles and solid phase with internal correlations

When the system consists of a heterogeneous mixture of polymeric agglomerates with mass-fractal-like scattering behavior, and solid randomly packed agglomerates that are constructed from the same primary scatterers, the intensity function can be written as a linear combination of the mass-fractal function (Equation (4.4)) and the structure function for packing of polydisperse spheres (Equation (4.6) and (4.10)). This approach was introduced to model the nanoscale structure evolution in drying barium titanium alkoxide carboxylate sol-gel films [32, 47]. Cross interference between both phases was neglected since it is probably a heterogeneous process consisting of a condensed phase and a dispersed phase that do not interact with each other. Together with the form function (Equation (4.2)), the scattering intensity of such mixtures can be described by:

$$
I_{\text {mix }}(q)=I_{0} \cdot\left(\eta \cdot S_{\mathrm{PC}}\left(q, \phi, \mu_{\mathrm{Ls}}, \sigma_{\mathrm{Ls}}\right)+(1-\eta) \cdot S_{\mathrm{MF}}\left(q, D, \xi, r_{0}\right)\right) \cdot P\left(q, r_{0}\right)
$$


Here $I_{0}$ is a scaling constant related to the scattering intensity at $q \rightarrow 0$, and $\eta$ is a dimensionless parameter with a value between 0 and 1 that describes to what extent the SAXS curve can be described in terms of correlated agglomerates. This parameter is hereafter referred to as the conversion coefficient. It is the ratio of the number of segregated secondary phase particles to the total number of scattering particles.

Since the model is relatively complex we have also determined model independent parameters that will support the observed trends in the previously described model, namely the invariant $Q_{\text {inv }}$ (Equation (4.14)) [15,37-38], the integral correlation length $L_{c}$ (Equation (4.15)), [37-38,51] and the correlation or Porod volume $V_{c}$ (Equation (4.16)) [37-38]. The invariant is a measure of the overall extent of electron density fluctuations in the sample, and $L_{c}$ and $V_{c}$ are average integral length and integral volume of the electron lean and electron dense domains within the measured region, respectively.

$$
\begin{gathered}
Q_{i n v} \equiv\left(\Delta n_{e}\right)^{2}=\int_{0}^{\infty} I(q) \cdot q^{2} d q \\
L_{C} \equiv \frac{\left\langle L_{i}^{2}\right\rangle}{\left\langle L_{i}\right\rangle}=\frac{\pi \cdot \int_{0}^{\infty} I(q) \cdot q \cdot d q}{Q_{i n v}} \\
V_{C} \equiv \frac{\left\langle V_{i}^{2}\right\rangle}{\left\langle V_{i}\right\rangle}=\frac{2 \pi^{2} \cdot \lim _{q \rightarrow 0}(I(q))}{Q_{i n v}}
\end{gathered}
$$




\subsection{Results and Discussion}

\subsubsection{Hydrolysis and condensation of mixed sol particles}

The small hybrid silica sol particles from curve a in Figure 1 could be well described by a Guinier approximation [38, 52]. Niobium penta-ethoxide (NPE) is very prone towards hydrolysis and a solution with $[\mathrm{NPE}]=0.54$ $\mathrm{mol} / \mathrm{L},[-\mathrm{OR}]:\left[\mathrm{H}_{2} \mathrm{O}\right]:\left[\mathrm{HNO}_{3}\right]=1: 1:(1 / 30)$ formed a gel within a few minutes. The corresponding scattering curve is curve $b$ in Figure 4.2. The scattering curve of the larger mixed niobia-hybrid silica particles in curve c could be described better as branched polymeric particles using the mass-fractal equation (4.4). Here a solution of NPE in ethanol ([NPE] $=0.54 \mathrm{~mol} / \mathrm{L}$ ) was added to a solution of prehydrolysed BTESE sol with a scattering curve as shown by curve a, while keeping the overall alkoxide group concentration constant at $[-\mathrm{OR}]=2.7 \mathrm{~mol} / \mathrm{L}$. It is noted that these sol particles should not be considered as "true" fractals, since self-similarity over length scales in these particles was limited. Nevertheless, it is the best theory available to describe the interparticle correlations in statistically branched polymeric assemblies, and has been applied successfully to describe other systems as well $[29,53]$. The equation could be fitted very well to the experimental SAXS curves and is based on only four free parameters $\left(I_{0}, D, r_{0}\right.$ and $\left.\xi\right)$, where $I_{0}$ is a scaling constant that depends on the number of electron density fluctuations and the contrast in electron density. Since the selfsimilarity across length scales was limited, the mass-fractal region was small and not very distinctive and the accuracy of the $D$-parameter was limited. However, the fit enabled us to determine a reliable value for $R_{\mathrm{g}}$ (Equation (4.5)). 


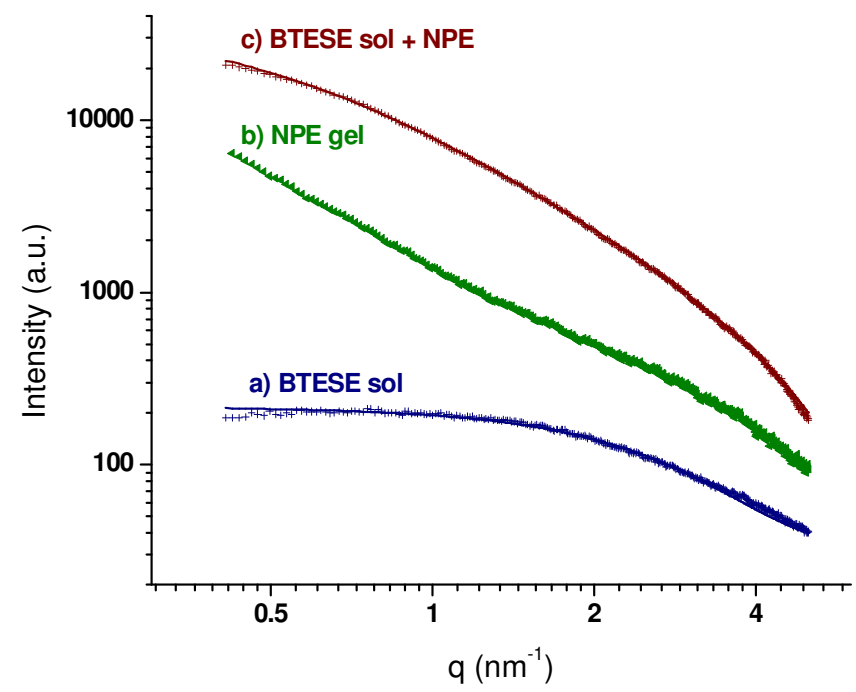

Figure 4.2. SAXS curves of sols. (a) BTESE sol after $1 \mathrm{~h}$ reflux at $60^{\circ} \mathrm{C}$; (b) Niobium penta-ethoxide (NPE) gel; (c) The BTESE sol after $1 \mathrm{~h}$ reflux at $60^{\circ} \mathrm{C}$ with NPE-solution added after the 1 hour and then measured after 2 minutes. For the sake of clarity, graphs $b$ and $c$ were shifted upwards by multiplication and their original shape remained intact.

After $1 \mathrm{~h}$ at $60^{\circ} \mathrm{C}$, the BTESE sol of curve "a" contained particles with an average radius of gyration $R_{\mathrm{g}}=0.49 \mathrm{~nm}$. In contrast to the NPE sol (curve b) instant gelation did not occur when a solution of NPE was added to the BTESE sol. The straight line at low $q$-values in the case of curve " $b$ " indicated that $R_{\mathrm{g}}$ is too large to measure within this q-range and should be larger than $10 \mathrm{~nm}$. When NPE was added to prehydrolyzed BTESE the resulting SAXS curve (curve " $c$ ") revealed the presence of larger particles in the sol with a cut-off distance $\xi=1.3 \mathrm{~nm}$ and fractal dimension $D=1.9$. The fitted values of $r_{0}$ became of the order of $\sim 0.05 \mathrm{~nm}$, which is physically impossible. This indicates that in this stage, the primary building blocks cannot be well described as spheres. Probably, these building blocks have a more polymeric and/or elongated shape. The $\xi$ value corresponds to a radius of gyration $R_{\mathrm{g}}=2.1 \mathrm{~nm}$. The presence of BTESE in the sol seems to inhibit the growth of niobium oxide building blocks and prevent their aggregation. The sols with shorter prehydrolysis times were almost identical to sample p60. 


\subsubsection{The film drying process}

The scattering intensity of a drying film of prehydrolyzed sol sample p60 as a function of drying time in air shown in Figure 4.3 represents a typical drying process. The SAXS curves are colored as a guide to the eye to distinguish the three basic stages of drying. In the initial stage of drying (blue curves) the shape of the SAXS patterns remained almost unchanged, except for the observed decrease of scattering intensity at high $q$. This decrease indicates that the smallest building blocks were disappearing. Since the region with constant slope in the $\log I-\log q$ plot was small at $q>$ $1.5 \mathrm{~nm}^{-1}$, the scattering curves are characteristic for small branched nanosized sol particles that contain a small number of building blocks. At $q<1.5$ $\mathrm{nm}^{-1}$ the curve flattened. This regime was well described by a Guinier approximation for spherical particles [42-43], which indicates that these dispersed sol particles had a limited size.

In the second stage of the drying process (green curves), ethanol that initially surrounded the sol particles had been replaced by other sol particles with similar electron densities due to solvent evaporation, resulting in diminishing $\Delta \rho$ between sol particles and their surrounding environment. The decrease of $\Delta \rho$ caused a strong decrease of scattering intensity, especially at low $q$-values. We visually observed the appearance of a separate solid phase in the drying sol within this same time frame. Since the liquid film was spread evenly over the continuously rotating rotorhead of the drying set-up, the scattering profile was an average of scattering contributions from the spatially separated sol phase and the newly forming condensed solid phase. At $t>20 \mathrm{~min}$ (red curves), most off the ethanol had evaporated and a clear correlation peak became visible in the SAXS pattern at $q^{\sim} 4.0 \mathrm{~nm}^{-1}$. This type of correlation peak suggests a repetitive separation distance of $2 \pi / q=1.5 \mathrm{~nm}$ between certain electron contrasted domains that correlate with each other within the agglomerated powder [12-13]. 


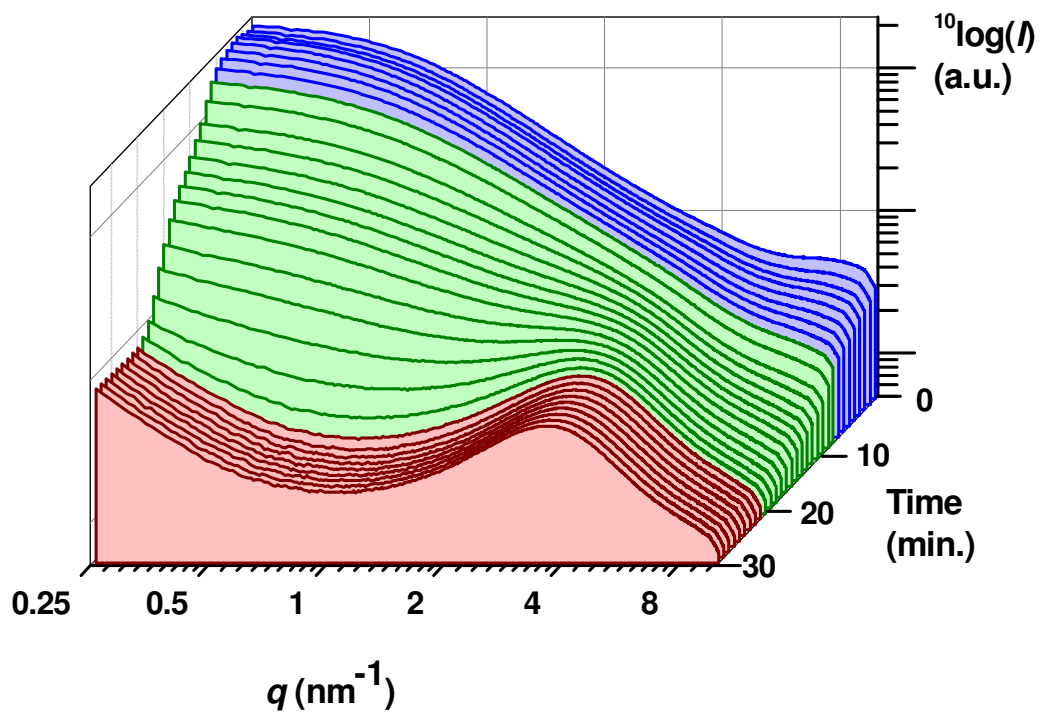

Figure 4.3. Evolution of SAXS curves during drying of thin film of sample p60 at $60^{\circ} \mathrm{C}$. Colors are a guide to the eye and indicate the three stages of drying as discussed in the text.

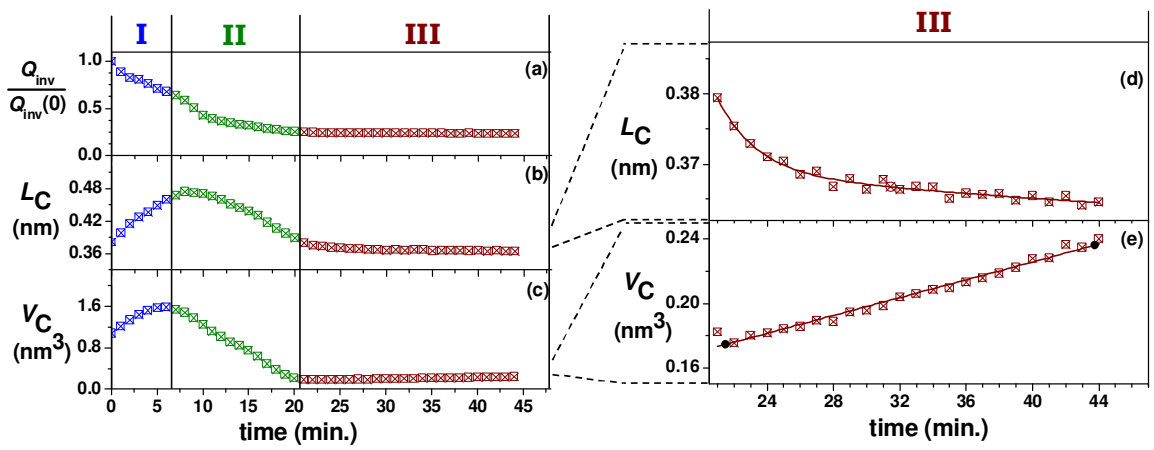

Figure 4.4. (a) The ratio of invariant $Q_{\text {inv }}$ and initial invariant $Q_{\text {inv }, t=0}$; (b) integral correlation length $L_{c} ;$ (c) correlation or Porod volume $V_{c}$ of sample p60 as a function of drying time at $T=60^{\circ} \mathrm{C}$ and in a set with more detail: (d) integral correlation length; (c) correlation volume in the final stage of the drying process. Colors are a guide to the eye and indicate the three stages of drying as discussed in the text. 
The model independent parameters, i.e. the invariant $Q_{\text {inv }}$ (Equation (4.14)), the correlation length $L_{C}$ (Equation (4.15), and the correlation or Porod volume $V_{c}$ (Equation (4.16)) were calculated (See Figure 4.4).

The regimes outside the measured $q$-range were approximated with a Guinier approximation $I \sim \exp \left(q^{2} R_{\mathrm{g}}{ }^{2} / 3\right)$ and Porod law $I \sim q^{-4}[36-38,54]$. We had performed two measurements in a regime at $\left(3<\mathrm{q}<32 \mathrm{~nm}^{-1}\right)$ [see Supplementary Information]. These measurements revealed correlation peaks that were associated with $\mathrm{Nb}-\mathrm{Nb}$ distances [55]. Since, this regime was dominated by atomic correlations we cannot verify the validity of Porod's law in this regime, however we cannot expect that this regime contains substantial information of the size of these building blocks either at these length scales. Thus, in order to gain some insight concerning the evolution of heterogeneous matrix as a simplified assembly of electron dense and electron poor regions we could use Porod's law. Moreover, in the following discussion we used the invariant $Q_{\text {inv }}$, the correlation length $L_{C}$ and Porod volume $V_{c}$ as a measure of electron dense and electron lean regions. The interpretation of these parameters became more straightforward when we ignore the fact that these regions were actually build from atoms and when we assume that these regions themselves have a homogeneous electron density distribution. Therefore, we neglect the correlation peaks at $\mathrm{q}>9 \mathrm{~nm}^{-1}$, since these correlations were associated with atomic correlations that overlap with the Porod regime of building blocks.

$Q_{\text {inv }}$ decreased gradually and leveled off after $20 \mathrm{~min}$, after which a dry film remained (Figure 4.4a). The trend reveals the reduction of $\Delta \rho$ as a result of physical loss of solvent as discussed above. The evolution of the integral correlation length $L_{c}$ reveals three stages: An initial (induction) stage in which $L_{c}$ increased slightly; a second (transition) stage during which $L_{C}$ decreased with time, and a final stage in which it remained constant. The increase of $L_{C}$ in the first stage coincides with a decrease in scattering intensity at high $q$ as discussed above, while the bending point at low $q$ remained unchanged. Thus, the primary building blocks from which the sol particles were assembled increased in size, while the sol particle size itself remained constant. The phenomenon can be understood in terms of Ostwald ripening, where the smallest entities with the highest curvatures 
dissolve. However, it may also involve rearrangements of entities, such that the building blocks became more spherical. In either case the system will reduce its overall surface energy and the sharpest curvatures will disappear. As the smallest features in a system disappear, the value of $L_{C}$ increases. The decrease in $L_{c}$ during the second stage coincided with the strong decrease in scattering intensity at low $q$. Since the sol particles approach each other, the contrast between particles and solvent diminished. Hence the distance correlation that was associated with the outer radius of these particles diminished as well. What remained is a correlation length that is associated only with the size of the building blocks that form the network. In the final stage, in which the network was consolidated, $L_{C}$ leveled off and remained constant. The correlation volume $V_{c}$ followed a similar but more pronounced trend as $L_{c}$.

The dried film consisted of alternating electron-rich and electron-lean regions. It is most probable that $L_{c}$ is a measure of the average size of these regions. The contrast in electron density can either be caused by a contrast between pores and matrix, or between niobium-rich and silicon-rich regions. The absence of mesopores was verified by both TEM and $\mathrm{N}_{2}$ adsorption. The $\mathrm{N}_{2}$ adsorption isotherm revealed only marginal $\mathrm{N}_{2}$ adsorption. Thus, if the material contained any pores, these were smaller than the kinetic diameter of nitrogen [see supporting information]. The TEM measurements were performed on a spincoated layer on a holey carbon grid, see Figure 4.5. The dried film was optically uniform (Figure 4.5a), except for a few exceptionally large spots (> $5 \mathrm{~nm}$ ), see Figure $4.5 \mathrm{~b}$. Besides the large spots, we observed many much smaller spots $(\leq 1 \mathrm{~nm})$, which can be associated with the presence of small electron-rich niobium centers. The sizes of these spots were close to the resolution of our TEM equipment. The amorphous region in Figure 4.5a contained both niobium and silicon, as shown by the EDX measurement in Figure 4.6a. The dark spots in Figure $4.5 \mathrm{~b}$ with the highest electron densities contained a higher concentration of niobium (Figure 4.6c) than the surrounding lighter regions (Figure 4.6b). We may therefore assign the correlation peak in the SAXS curve to the presence of alternating regions that are enriched in either niobia or hybrid silica. 


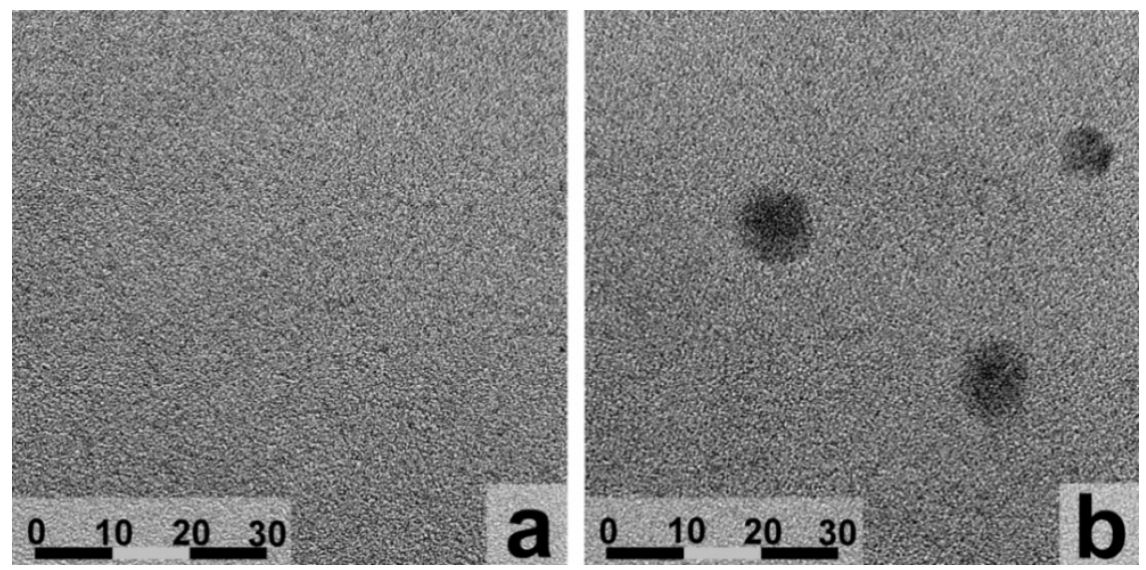

Figure 4.5. TEM image of dried film sample p60.(a) General overview; (b) Examples of anomalously large dark spots. The scale bar represents the scale in $\mathrm{nm}$.

(a)

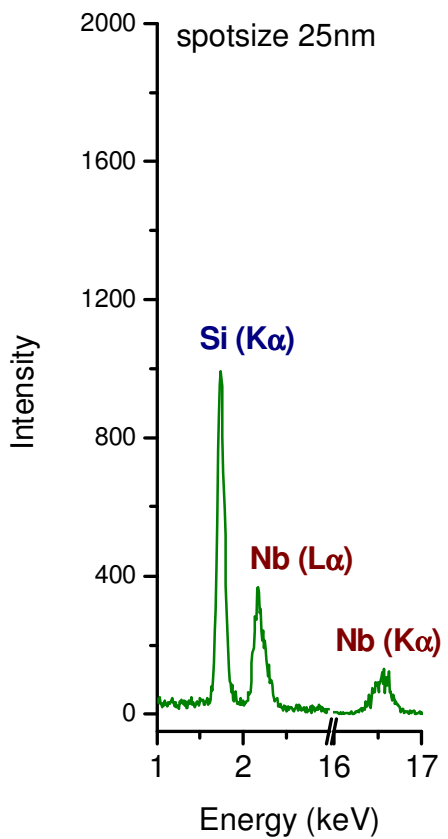

(b)

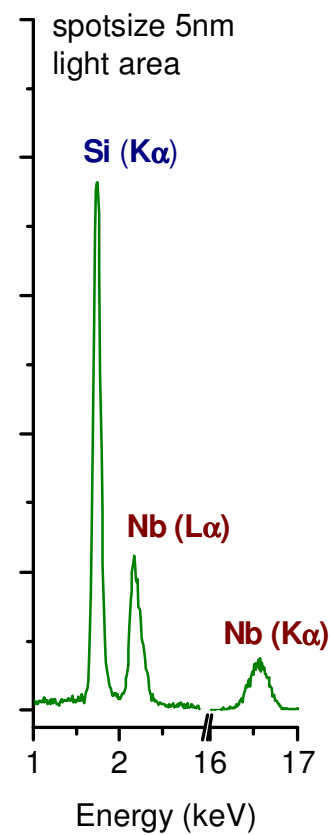

(c)

spotsize $5 \mathrm{~nm}$ dark area

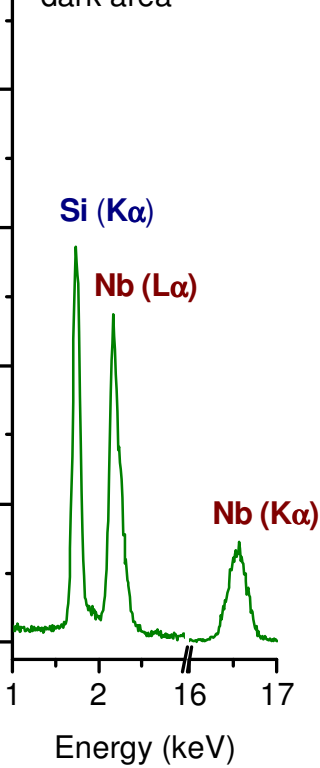

Figure 4.6. EDX analysis of as-dried film p60. (a) Measurement with $25 \mathrm{~nm}$ spotsize in Figure 4.5a; (b) Measurement of $5 \mathrm{~nm}$ spot in light area of Figure 4.5b; (c) Measurement of $5 \mathrm{~nm}$ spot in dark area of Figure 4.5b. 
To account for the observed phenomena and SAXS profiles, we propose in Figure 4.7a schematic representation of the structure evolution process on nanometer-scale during reaction and film drying that is consistent with the experimental data. First the BTESE sol hydrolyzed and condensed, forming small sol particles (Figure 4.7a) with $R_{\mathrm{g}}=0.49 \mathrm{~nm}$. Then NPE was added, which formed small particles and reacted with the pre-hydrolyzed BTESE (Figure 4.7b). Then the sol was dried as a thin film. During the first stage of drying, the slope of the SAXS curve at high $q$ in Figure 4.3 became steeper. This indicates that the Porod region (where $I \sim q^{-4}$ ) shifted effectively to lower $q$, showing that the size of the primary building blocks increased (Figure 4.7b and Figure 4.6c). Probably, in this stage the smallest entities dissolved via Ostwald ripening. This explained the reduction of the overall number of electron density fluctuations, which is represented by $Q_{\text {inv }}$ (Figure 4.4a) as well as it explained increasing average sizes of electron rich and electron lean domains as represented by $V_{c}$ and $L_{c}$ (Figure $\left.4.4 b, c\right)$.

In the second stage of drying (green curves in Figure 4.3) the average distance between sol particles decreased, thereby decreasing the electron density contrast between sol particles and their surroundings. A solid phase with a correlation peak in its SAXS curve developed (Figure 4.7c and $6 \mathrm{~d}$ ). This process typically occurs on length scales $>1 \mathrm{~nm}$, and was confirmed by the decreasing scattering intensity at low $q$ in Figure 4.3. The number of correlations at these length scales reduced and therefore the average length scale as indicated by both $V_{c}$ and $L_{c}$ (Figure $4.4 b, c$ ) reduced as well. However, the still ongoing autonomous growth of the primary building blocks counteracted this effect to some extent. The maximum value of both $V_{c}$ and $L_{c}$ was reached when both effects counterbalanced. After that moment, the reduction of $\Delta \rho$ between sol particles and surroundings became dominant, so that both $V_{c}$ and $L_{c}$ decreased. Since $V_{c}$ is a volumeweighted term and $L_{C}$ is a length-weighted term, $V_{c}$ is less sensitive to the increase of primary segment radius. The precise moment when the above mentioned effects counterbalance occurred earlier in time for $V_{c}$ than for $L_{C}$.

Finally, the trends in $Q_{\text {inv }}, L_{c}$ and $V_{c}$ leveled off in the third stage. After $t=24$ $\min L_{C}$ still decreased slowly (Figure 4.4b,d). Most likely, the capillary forces that were developed during drying caused further densification of the solid phase and reduction of the building block-to-building block distances. On 
the other hand, $V_{c}$ is sensitive to large distance fluctuations. These were slightly increasing, as shown by the increasing slope at low $q$-values in Figure 4.3 (red curves). This suggests an increasing degree of disorder in the building block-to-building block packing distance upon drying.

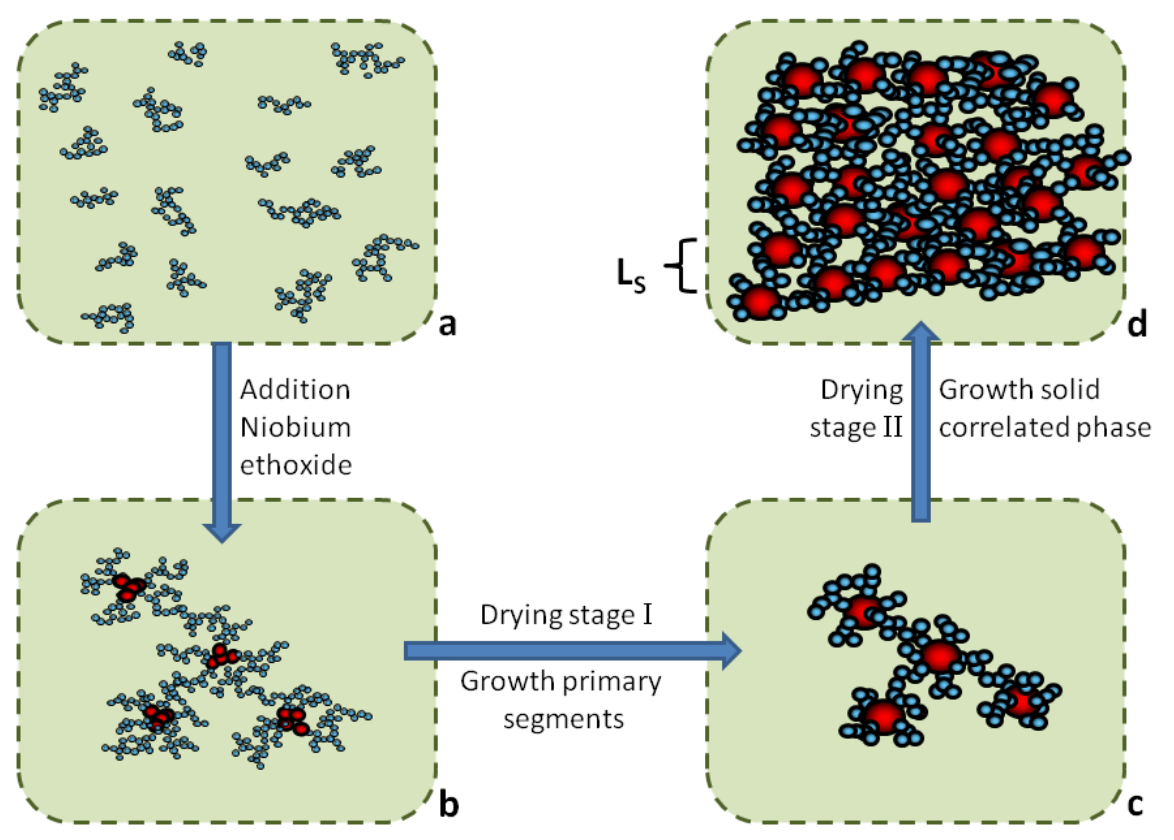

Figure 4.7. Schematic representation of proposed mechanism of condensation and aggregation of mixed niobia/hybrid silica during drying of thin film sols.

In the second stage of the drying process, we visually observed the formation of a secondary solid phase. Thus, in this stage a heterogeneous system was present in the film, in which a solid phase developed from a nanosized sol. The phase formation and separation process was accompanied by the formation of a characteristic SAXS correlation peak that represented particular distances between electron-rich domains. Both the dispersed sol phase and the newly formed solid phase had a characteristic integral correlation length $L_{c}$. After a short induction period during which nucleation sites for the solid phase formed, the solid phase 
continued growing from the solid-liquid interface. Therefore, the scattering profile was dominated by both the dispersed and the solid phases. We can define a conversion parameter, $\alpha_{L C}$ that quantifies the degree of transition from the initial polymeric sol with correlation length $L_{c}{ }^{p}$ into a solid phase consisting of a correlated stack of similarly-sized building blocks with a typical correlation length $L_{C}{ }^{C}$ (Equation (4.17)). Here $L_{C}{ }^{p}$ is defined as the correlation length before the transition stage (at the beginning of stage II) and $L_{C}{ }^{C}$ as the correlation length after the transition (at the end of stage III). Analogously, we can define a similar conversion parameter $\alpha_{v c}$ for the correlation volume ((4.18). The two resulting conversion parameters $\alpha_{\mathrm{Lc}}$ and $\alpha_{v_{c}}$ read:

$$
\begin{aligned}
& \alpha_{L c}(t)=\frac{L_{C}^{P}-L_{C}(t)}{L_{C}^{P}-L_{C}^{C}} \\
& \alpha_{V c}(t)=\frac{V_{C}^{P}-V_{C}(t)}{V_{C}^{P}-V_{C}^{C}}
\end{aligned}
$$

The corresponding curves in Figure 4.8 follow a typical sigmoidal trend. After an induction period in which ethanol evaporated (Figure 6b), the particle solution became supersaturated in concentration, which facilitated the agglomeration process (Figure 6c). A solid phase with characteristic correlation peak in its SAXS curve grew until $t \sim 20 \mathrm{~min}$. There $\alpha_{\mathrm{Lc}}$ and $\alpha_{V c}$ were close to 1 , showing that the conversion was nearly complete. The phase transformation rate can be fitted to the Johnson-Mehl-AvramiKolmogorov (JMAK) equation [56], which was developed to characterize crystallization processes, but has also been applied to describe the growth of silica [57] and the solidification of glasses [58-60]

$$
\alpha=A \cdot\left[1-\exp \left(-k \cdot\left(t-t_{i}\right)^{d}\right) \mid\right.
$$

Here $A$ is a normalization constant, $k$ a reaction rate constant, $d$ a parameter that is characteristic for a particular growth mechanism, and $t_{i}$ is an induction time that expresses the moment that conversion starts. We found similar JMAK-parameters for both conversion coefficients except for 
$t_{\mathrm{i}}$, which was $4.3 \pm 0.4 \mathrm{~min}$ and $1.3 \pm 0.5 \mathrm{~min}$ for $\alpha_{\mathrm{Lc}}$ and $\alpha_{V c}$, respectively. These times are somewhat before the moment $t_{\max }$ at which we find the maxima in $V_{\mathrm{C}}$ and $L_{\mathrm{C}}$. While $t_{\mathrm{i}}$ indicates the onset of the transition process towards a condensed phase, $t_{\max }$ is also related to growth effects of the primary particles, as discussed above. For both conversion coefficients, $d=$ $3 \pm 0.4$ which indicated that the packing of sol particles was associated with a polyhedral growth mechanism [56]. This means that the sol particles became randomly packed in all three dimensions. The other parameters were $k=(4.4 \pm 0.1) \cdot 10^{-4}$ and $(4.6 \pm 0.1) \cdot 10^{-4} \mathrm{~min}^{-\mathrm{d}}$, and $\mathrm{A}=0.99 \pm 0.01$ and $0.98 \pm 0.01$ for $\alpha_{L c}$ and $\alpha_{V c}$, respectively.

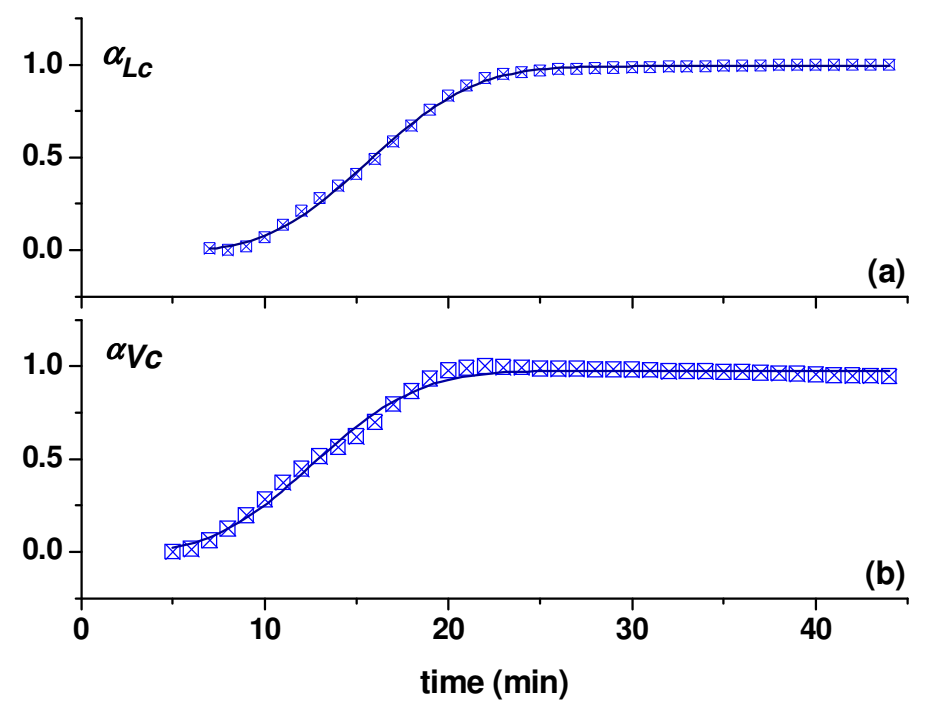

Figure 4.8. The conversion of a sol towards a dried gel of sample p60 during film drying at $T=60^{\circ} \mathrm{C}$, characterized by evolution of (a) correlation length $L_{c}$, and (b) correlation volume $V_{\mathrm{c}}$. The drawn lines indicate the best fits to the JMAK equation.

\subsubsection{Modeling the film drying process}

As analyzed above, the initial sol consisted of branched small particles and the corresponding SAXS curve could be described well in terms of a massfractal ((4.4). Upon drying, a solid phase developed, and a correlation peak showed up in the SAXS curve, which could be assigned to niobium-rich 
regions inside a hybrid silica matrix. Since we have no indications that niobia building blocks have a preferential growth direction we assumed that the building blocks were approximately spherical. Since long range order was absent, liquid-like random packing of building blocks is the most plausible interpretation of the correlation peak [40]. The niobia building blocks were dispersed in a hybrid silica matrix that kept them separated from each other. The nanostructure of the solid phase can thus be characterized in terms of length scales, namely the core radius $\left(r_{0}\right)$ of the niobium-rich building blocks (i.e. primary scatterers), and the mean building block-to-buiding block distance $\left(\mu_{\mathrm{Ls}}\right)$ from the correlation peak. The scattering intensity of the solid phase can therefore be described by Equation (4.7). Howevers, since the correlation peak was rather broad, we included the effect of polydispersity by the local monodisperse approximation (Equation ((4.10)). During the film drying process we assume the presence of a spatially separated mixture of a solid phase with internal structural correlations, and a polymeric sol in an ethanol matrix.

We optimized the model parameters $\left(I_{0}, \eta, \xi, D, \phi, \mu_{L s}, \sigma_{L s}, r_{0},(4.13)\right.$ by applying simplex [61] iterations until the correlation coefficient, $R_{\text {corr }}$, was 0.998 or higher. However, at $t<16 \mathrm{~min}$ we did not find a clear evolution of the conversion parameter, $\eta$. This was due to the absence of a correlation peak at $t<16 \mathrm{~min}$, so that the distinction that could be made between correlated aggregates (solid phase) and polymeric sol particles was poor. In order to be able to fit the data properly, we needed a proper approximation of the extent of conversion as characterized by $\eta$. As was demonstrated above, the conversion from sol into a solid was accompanied by a clear change in $V_{c}$ (Figure 4.4c). Therefore, the change of in $V_{c}$ expressed in terms of $\alpha_{v c}$ provides a proper estimate of $\eta$. When taking into account that $\alpha_{v c}$ is a volume-weighted coefficient and $\eta$ is a number-weighted conversion coefficient, then we can approximate $\eta$ by:

$$
\eta(t) \approx \frac{\alpha_{V c}(t) / V_{C}^{C}}{\alpha_{V c}(t) / V_{C}^{C}+\left(1-\alpha_{V c}(t)\right) / V_{C}^{P}}=\frac{1}{1+\left(\frac{1-\alpha_{V c}(t)}{\alpha_{V c}(t)}\right) \cdot \frac{V_{C}^{C}}{V_{C}^{P}}}
$$


The remaining seven parameters $\left(I_{0}, \xi, D, \phi, \mu_{L s,} \sigma_{L s,} r_{0,}\right.$ (4.13) were optimized by fitting to the experimental curves. The best fits matched well with the experimental SAXS curves, with correlation coefficients $R_{\text {corr }}$ of at least 0.999. See Figure 4.9. The modeled data only deviated from the experimental data in the initial stage of the drying process, at $t=0-5 \mathrm{~min}$, where the scattering intensity leveled off above $q \sim 4 \mathrm{~nm}^{-1}$. The data in that small time frame could not be described well using a spherical form factor. The quality of fit might be improved by an ellipsoid or cylindrical form factor, but since it would increase the complexity of our model, and was not expected to provide further insight, we did not attempt to improve these fits in the time frame between 0 and $5 \mathrm{~min}$.

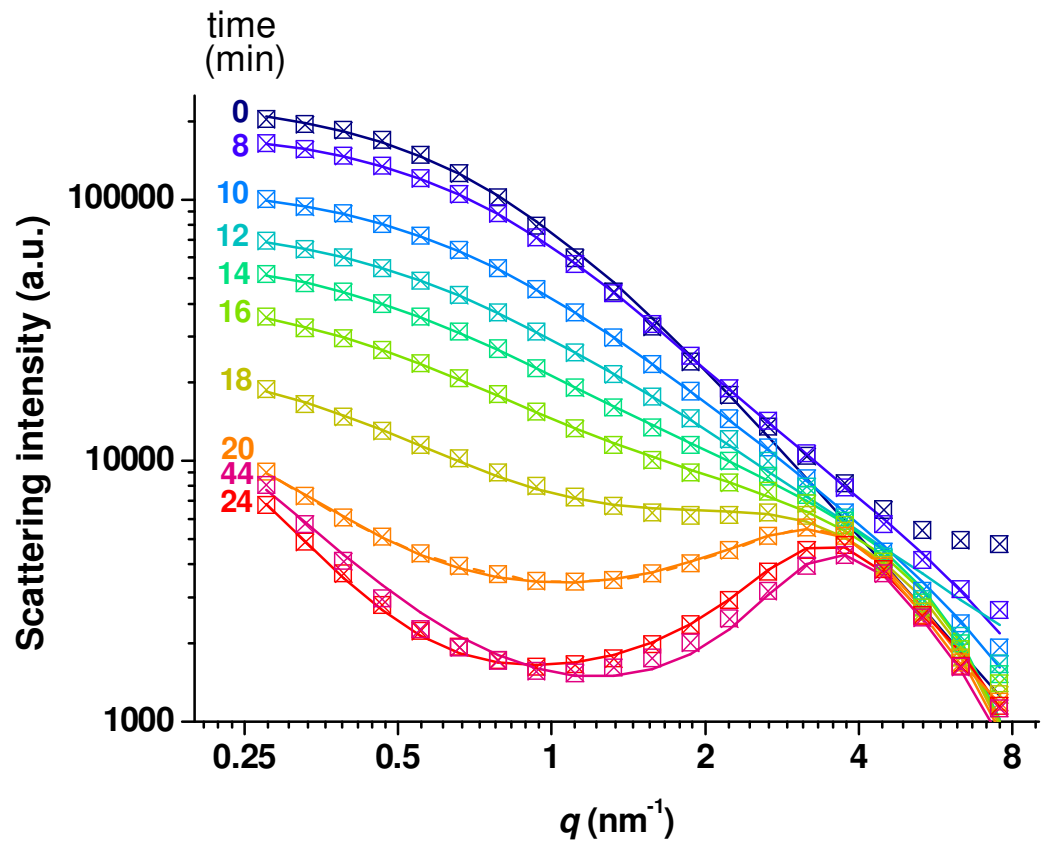

Figure 4.9. The modeled SAXS curves at several timescales while drying sample p60 at $\mathrm{T}=60^{\circ} \mathrm{C}$ using (4.13. The conversion parameter ${ }_{V}$ was calculated from (4.20. Colors are a guide to the eye. 

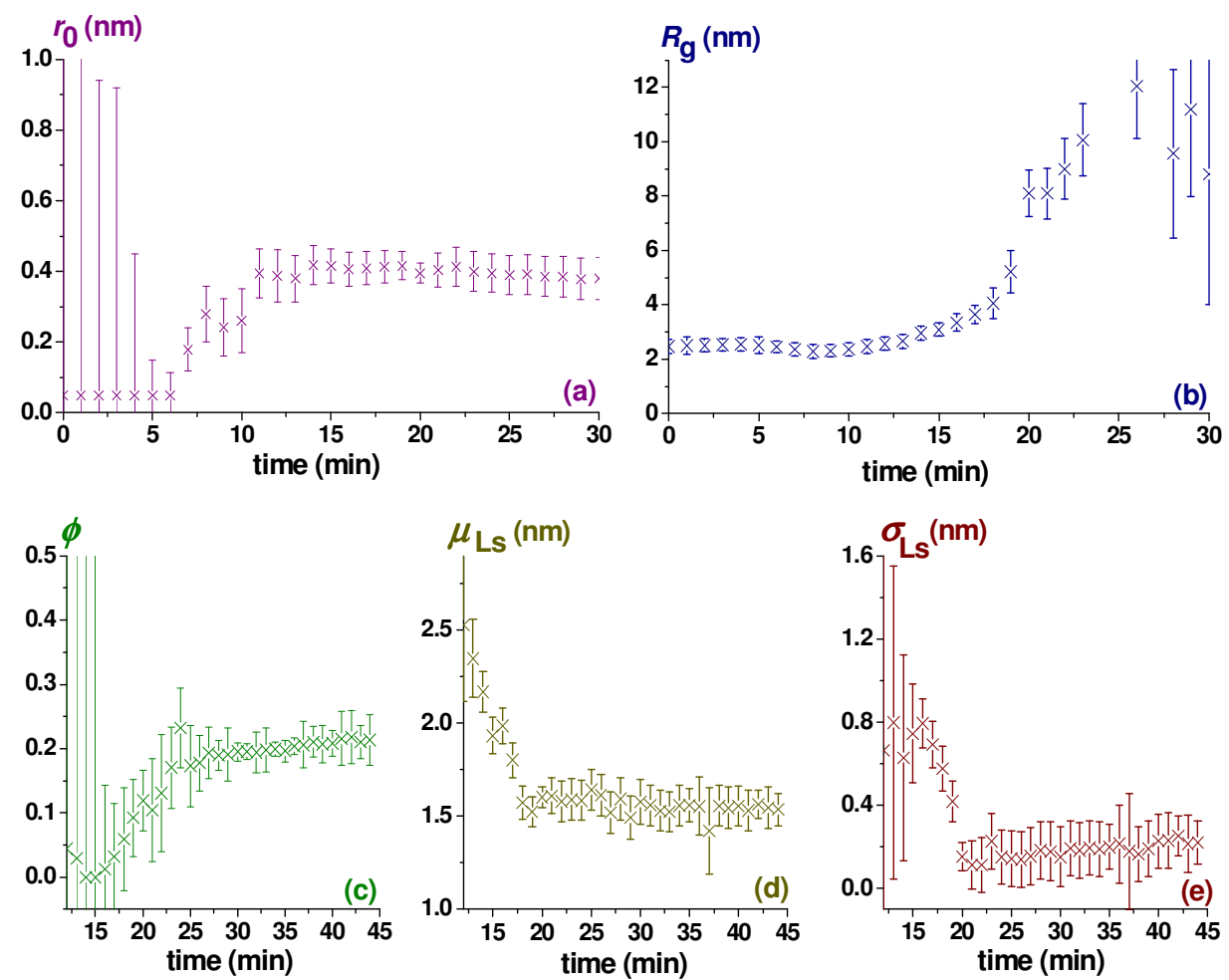

Figure 4.10. Parameters from fitting drying sample p60 at $\mathrm{T}=60^{\circ} \mathrm{C}$ using Equation (4.13). (a) Primary particle radius $r_{0}$; (b) radius of gyration of sol particles $\boldsymbol{R}_{\mathrm{g}}$; (c) building block volume fraction in the local surroundings of a building block $\phi$, (d) intensity-averaged building block-to-building block distance $\mu_{\mathrm{Ls}}$; (e) mean square deviation in distribution of building block-tobuilding block distance $\sigma_{\mathrm{Ls}}$.

At $t>8$ min the curves could be fitted using a spherical form function and $r_{0}$ increased to $\sim 0.4 \mathrm{~nm}$ (Figure 4.10a). The increase coincided with an increase of $V_{c}$, and may be related to Ostwald ripening as discussed above. At $t>12 \mathrm{~min}, r_{0}$ leveled off. The size of the building blocks is similar to the size of the niobia core of a $\mathrm{Nb}_{8} \mathrm{O}_{10}(\mathrm{OEt})_{20}$ complex [62]. The $\mathrm{Nb}_{8} \mathrm{O}_{16}$ core has an radius of gyration $R_{\mathrm{g}}$ of $0.33 \mathrm{~nm}$, which corresponds to a spherical crosssectional radius of $r_{0}=R_{\mathrm{g}}(5 / 3)^{1 / 2}=0.42 \mathrm{~nm}$ [37]. In the calculation of latter value we discarded the surrounding carbon atoms and pendant oxygen atoms since these have a similar electron density as ethanol, and can 
therefore not be distinguished from the solvent matrix. Within the first 15 $\mathrm{min}$, the radius of gyration of the polymeric units remained approximately constant at $R_{\mathrm{g}}=1.5 \mathrm{~nm}$ (Figure $4.10 \mathrm{~b}$ ). Then $R_{\mathrm{g}}$ increased rapidly to $R_{\mathrm{g}} \sim$ $8.5 \mathrm{~nm}$. At $t>20 \mathrm{~min}$ the bending point in the SAXS curve moved outside the $q$-range covered by our equipment, and $R_{\mathrm{g}}$ became too large to determine. Its value can be considered close to infinite as soon as all ethanol has evaporated. At $t>15 \mathrm{~min}$ a correlation peak appeared in the SAXS curves. From this moment onwards, the local volume fraction $\phi$, mean building block-to-building block distance $\mu_{\mathrm{Ls}}$, and its standard deviation $\sigma_{\mathrm{Ls}}$ of $L_{\mathrm{s}}$ were determined by fitting to $(4.13$, see Figure $4.10 \mathrm{c}-\mathrm{e}$. At $t=17-22 \mathrm{~min}$ the extent of correlation between the electron-rich niobium building blocks clearly increased, as indicated by the increase of $\phi$. The majority of building blocks formed a separate solid phase. At $t=12-19 \mathrm{~min}$ the building blockto-building block distance $\mu_{\mathrm{Ls}}$ clearly decreased, while the typical size $r_{0}$, which was mainly determined by the size of the electron-rich $\mathrm{Nb}$-based building blocks remained constant from $t=12 \mathrm{~min}$. onwards. Because the solvent between the building blocks evaporated, the available volume in which building blocks could move freely became smaller as well. Therefore, the standard variation of building block-to-building block distance $\sigma_{L S}$ decreased simultaneously with $\mu_{\mathrm{Ls}}$.

In the third and final stage of the drying process, both $r_{0}$ and $\mu_{\mathrm{Ls}}$ decreased slightly. Since the solvent had already evaporated, this suggests a densification process of the as-formed solid phase. Moreover, $\phi$ increased, indicating increased interference between different niobia building blocks. In other words, since the building blocks are approaching each other the probability that a correlation between building blocks is found increased in the course of time. On the other hand, $\sigma_{\mathrm{Ls}}$ slowly increased, indicating an increased degree of disorder in $\mu_{\mathrm{Ls}}$.

\subsection{Effect of prehydrolysis of silica}

The time during which we hydrolyzed BTESE before adding the NPEprecursor (i.e. pre-hydrolysis time) was $60,30 \mathrm{~min}$ and $0 \mathrm{~min}$ in samples p60, p30 and p00, respectively. The radius of gyration after $1 \mathrm{~h}$ of reaction time decreased to $2.2,2.0$, and $1.5 \mathrm{~nm}$, respectively, as determined from fitting data to the mass-fractal equations (4.4) and (4.5). The initial integral correlation length $L_{c}$ of the simultaneously hydrolyzed sample p00 was substantially larger compared to the pre-hydrolyzed samples p30 and p60, see Figure 4.11a. All samples showed the same general trend: first, an 
increase of $L_{C}$ due to the increasing size of primary building blocks, followed by a decrease since the contrast between the polymeric agglomerates and their surroundings disappeared. The difference in $L_{C}$ between samples p30 and p60 was marginal although the radius of gyration of p30 was substantially larger than $\mathrm{p} 60$. The correlation volume $V_{c}$, which is dominated by the largest features in the system, revealed that the second stage, at which $V_{c}$ decreases, starts straightaway for sample p00. See Figure 4.11b. The final correlation volume was mainly determined by remaining polymeric aggregates and was found to be independent of the actual synthesis procedure.
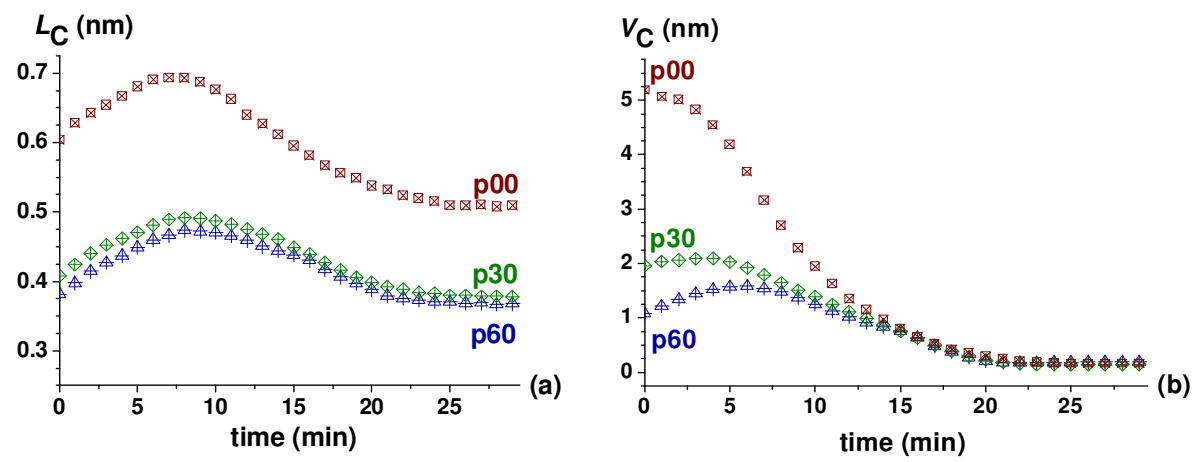

Figure 4.11.(a) Integral correlation length; (b) correlation or Porod volume with varying prehydrolysis time of BTESE of samples p00, p30, p60 as a function of drying time at $T=60^{\circ} \mathrm{C}$.

In samples p00 and p30 a sharp transition in $r_{0}$ is observed in Figure 4.12a. However, it was difficult to find a unique solution from the experimental data and the sharp transition was in disagreement with the smooth transition that we observed for $L_{c}$ and $V_{c}$ in the same period of time (Figure 4.11). After the second stage of drying process the values of $r_{0}$ and $L_{C}$ of samples p30 and p60 were almost identical. This means that although the initial radius of gyration of p30 was clearly larger compared to p60, the size of and distances between niobia-rich building blocks were similar after 20 min. In sample p00 both $r_{0}$ and $\mu_{\mathrm{Ls}}$ were substantially larger than in samples p30 and p60. 

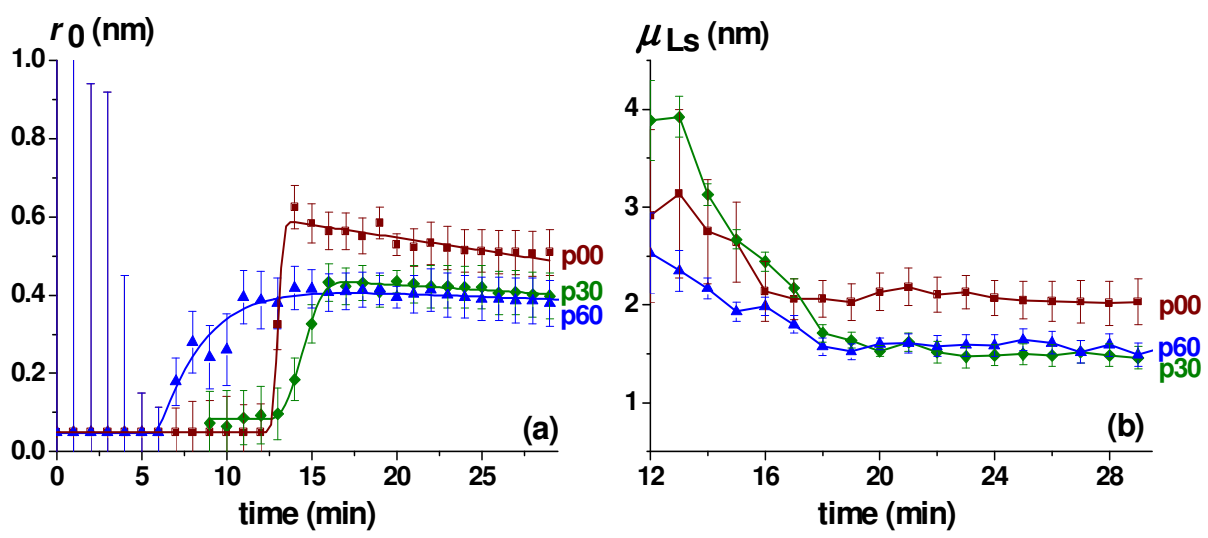

Figure 4.12. (a) Primary particle radius $r_{0}$ of samples p00, p30 and p60 with different prehydrolysis times of BTESE; (b) Intensity averaged building block-to-building block distance $\mu_{\mathrm{Ls}}$ of the same samples.

\subsection{The effect of drying temperature}

When the drying temperature was decreased from $80{ }^{\circ} \mathrm{C}$ to lower temperatures, the change of both $r_{0}$ and $\mu_{\mathrm{Ls}}$ became more gradual and the induction period increased. See Figure 4.13. However, the trends are similar. After an induction period, $r_{0}$ increased rapidly, followed by slow densification during which $r_{0}$ decreased slowly. For the sake of clarity, the initial $r_{0}$ values were removed from the graph, since the error margins were rather large. Within the parameter uncertainties the sizes of these building blocks were not substantially different after solvent evaporation, i.e. $r_{0} \sim$ $0.4 \mathrm{~nm}$. Probably, the drying temperature only has a minor influence on the size of the niobium-rich regions. Their size was probably established at the moment when the NPE precursor was added to the pre-hydrolyzed BTESE sol. The scattering intensity in that stage was dominated by the electron density contrast between both types of oxide building blocks and the solvent phase around these building blocks. The value of $r_{0}$ was determined by the sizes of the niobia and hybrid silica building blocks. After evaporation of ethanol, the contrast between the electron-rich niobia and electron-poor hybrid silica became the major contribution to the overall contrast in 
electron density. This means that these niobia building blocks were not necessarily absent before the drying process started and their presence became more prominently visible when the solvent evaporated.
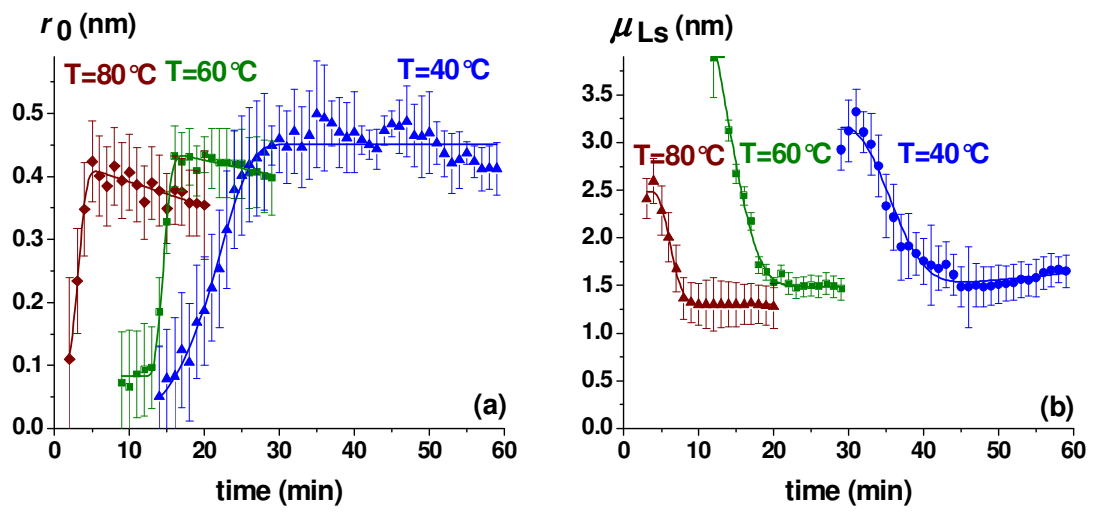

Figure 4.13. Evolution of (a) $r_{0}$ and (b) $\mu_{\mathrm{Ls}}$ at 3 drying temperatures, $\mathrm{T}=40$, 60 and $80^{\circ} \mathrm{C}$.

\subsection{Conclusions}

The drying process of sol-gel derived niobia-hybrid silica mixed oxide thin films can be divided into three different stages. The first is an induction stage during which the primary building blocks were growing while the overall sol particle remained constant in size. The shape of the sol particles could be described in terms of a mass-fractal model. During this stage the solvent concentration in the system was high. The second represents a transition during which sol particles approach each other as a result of ethanol evaporation. In the third stage, a densification process takes place in the as-dried film after solvent evaporation, which is accompanied by some small structural reconstructions.

Before the transition process started, the SAXS curve was mainly determined by the electron density contrast between sol particles and surrounding ethanol matrix. During the transition process this contribution was reduced to zero due to solvent evaporation. However, electron density contrast between small molecular niobium-rich building blocks and the 
surrounding hybrid silica matrix remained. This finally led to a correlation peak in the SAXS curves. The position of this peak was determined by the building block-to-building block distances between the small niobia building blocks. Building block sizes were extracted using hard sphere form functions and structural interference functions that were based on a Percus-Yevick approximation of hard spheres. When hybrid silica was hydrolyzed before adding NPE (samples p30 at three different drying temperatures and sample p60), all contained niobium-rich building blocks with a radius of $0.4 \mathrm{~nm}$, which is of the order of the size of an $\mathrm{Nb}_{8} \mathrm{O}_{16}$ core. The sample p00 for which NPE was added at the beginning of the sol preparation process gave rise to niobium-rich building blocks with a larger average radius of 0.5 $-0.6 \mathrm{~nm}$. This shows that hydrolysis of the hybrid silica sol before NPE addition gives rise to smaller niobium-rich building blocks in the mixed oxide film after drying.

The drying temperature influenced the rate at which the sol was dried. However, it had a negligible effect on the size of the niobium-rich building blocks. The size of these building blocks is thus likely established during the sol preparation process and not during drying.

\subsection{References}

1. Vane, L. M., A review of pervaporation for product recovery from biomass fermentation processes. J. Chem. Technol. Biotechnol. 2005, 80(6), p.603-629.

2. van Gemert, R. W.; Petrus Cuperus, F., Newly developed ceramic membranes for dehydration and separation of organic mixtures by pervaporation. J. Membr. Sci. 1995, 105(3), p. 287-291

3. Urtiaga, A. M.; Gorri, E. D.; Gómez, P.; Casado, C.; Ibáñez, R.; Ortiz, I., Pervaporation Technology for the Dehydration of Solvents and Raw Materials in the Process Industry. Drying Technol. 2007, 25 (11), p. 1819-1828.

4. Castricum, H. L.; Sah, A.; Kreiter, R.; Blank, D. H. A.; Vente, J. F.; Ten Elshof, J. E., Hydrothermally stable molecular separation membranes from organically linked silica. J. Mater. Chem. 2008, 18(18), p. 2150-2158. 
5. Kreiter, R.; Rietkerk, M. D. A.; Castricum, H. L.; Van Veen, H. M.; Ten Elshof, J. E.; Vente, J. F., Evaluation of hybrid silica sols for stable microporous membranes using high-throughput screening. J. SolGel Sci. Technol. 2011, 57 (3), p. 245-252.

6. Fotou, G. P.; Lin, Y. S.; Pratsinis, S. E., Hydrothermal stability of pure and modified microporous silica membranes. J. Mater. Sci. 1995, 30 (11), p. 2803-2808.

7. Yoshida, K.; Hirano, Y.; Fujii, H.; Tsuru, T.; Asaeda, M., Hydrothermal stability and performance of silica-zirconia membranes for hydrogen separation in hydrothermal conditions. J. Chem. Eng. Jpn. 2001, 34 (4), p. 523-530.

8. Asaeda, M.; Sakou, Y.; Yang, J.; Shimasaki, K., Stability and performance of porous silica-zirconia composite membranes for pervaporation of aqueous organic solutions. J. Membr. Sci. 2002, 209(1), p. 163-175.

9. Boffa, V.; Blank, D. H. A.; ten Elshof, J. E., Hydrothermal stability of microporous silica and niobia-silica membranes. J.Membr. Sci. 2008, 319(1-2), p. 256-263.

10. Kanezashi, M.; Asaeda, M., Hydrogen permeation characteristics and stability of Ni-doped silica membranes in steam at high temperature. J. Membr. Sci. 2006, 271 (1-2), 86-93.

11. Igi, R.; Yoshioka, T.; Ikuhara, Y. H.; Iwamoto, Y.; Tsuru, T., Characterization of co-doped silica for improved hydrothermal stability and application to hydrogen separation membranes at high temperatures. J. Am. Ceram. Soc. 2008, 91(9), p. 2975-2981.

12. Gaudon, A.; Dauger, A.; Lecomte, A.; Soulestin, B.; Guinebretière, $\mathrm{R} .$, Phase separation in sol-gel derived $\mathrm{ZrO2-SiO2} \mathrm{nanostructured}$ materials. J. Eur. Ceram. Soc. 2005, 25(2-3 SPEC. ISS.), p. 283-286.

13. Rodrigues, D. E.; Brennan, A. B.; Betrabet, C.; Wang, B.; Wilkes, G. L., Structural features of sol-gel-derived hybrid inorganic-organic network ceramer materials by small-angle $x$-ray scattering. Chem. Mater. 1992, 4(6), p. 1437-1446;

14. Dahmouche, K.; Santilli, C. V.; Pulcinelli, S. H.; Craievich, A. F., SmallAngle X-ray Scattering Study of Sol-Gel-Derived Siloxane-PEG and Siloxane-PPG Hybrid Materials. J. Phys. Chem. B 1999, 103(24), p. 4937-4942.

15. Brinker, C. J. S., G. W. , Sol Gel Science, The Physics and Chemistry of Sol-Gel Processing. Acedemic Press: San Diego, 1990; Vol. 1, p. 1881. 
16. Kessler, V. G.; Spijksma, G. I.; Seisenbaeva, G. A.; Håkansson, S.; Blank, D. H. A.; Bouwmeester, H. J. M., New insight in the role of modifying ligands in the sol-gel processing of metal alkoxide precursors: A possibility to approach new classes of materials. J. SolGel Sci. Technol. 2006, 40(2-3), p. 163-179.

17. Drake, K. O.; Carta, D.; Skipper, L. J.; Sowrey, F. E.; Newport, R. J.; Smith, M. E., A multinuclear solid state NMR study of the sol-gel formation of amorphous $\mathrm{Nb}_{2} \mathrm{O}_{5}-\mathrm{SiO}_{2}$ materials. Solid State Nucl. Magn. Reson. 2005, 27(1-2), p. 28-36.

18. Bansal, N. P., Synthesis and thermal evolution of structure in alkoxide-derived niobium pentoxide gels. J. Mater. Sci. 1994, 29(17), p. 4481-4486.

19. Kreiter, R.; Rietkerk, M. D. A.; Castricum, H. L.; Van Veen, H. M.; Ten Elshof, J. E.; Vente, J. F., Stable hybrid silica nanosieve membranes for the dehydration of lower alcohols. ChemSusChem 2009, 2(2), p. 158-160.

20. lizuka, T.; Ogasawara, K.; Tanabe, K., Acidic and catalytic properties of niobium pentaoxide. Bull. Chem. Soc. Jpn. 1983, 56(10), p. 29272931.

21. Prakash, A. M.; Kevan, L., Synthesis of niobium silicate molecular sieves of the MFI structure: Evidence for framework incorporation of the niobium ion. J. Am. Chem. Soc. 1998, 120(50), p. 13148-13155.

22. Nowak, I.; Ziolek, M., Niobium Compounds: Preparation, Characterization, and Application in Heterogeneous Catalysis. Chem. Rev. 1999, 99(12), p. 3603-3624.

23. Guerrero, S.; Miller, J. T.; Wolf, E. E., Activity and selectivity control by niobium for the preferential oxidation of Co on Pt supported catalysts. Appl. Catal. A 2007, 328(1), p. 27-34.

24. Tanabe, K., Application of niobium oxides as catalysts. Catal. Today 1990, 8(1), 1-11.

25. (a) Srilatha, K.; Lingaiah, N.; Sai Prasad, P. S.; Prabhavathi Devi, B. L. A.; Prasad, R. B. N.; Venkateswar, S., Influence of Carbon Chain Length and Unsaturation on the Esterification Activity of Fatty Acids on $\mathrm{Nb}_{2} \mathrm{O}_{5}$ Catalyst. Ind. Eng. Chem. Res. 2009, 48(24), p. 1081610819; (b) Srilatha, K.; Lingaiah, N.; Devi, B. L. A. P.; Prasad, R. B. N.; Venkateswar, S.; Prasad, P. S. S., Esterification of free fatty acids for biodiesel production over heteropoly tungstate supported on niobia catalysts. Appl. Catal. A, 2009, 365(1), p. 28-33. 
26. West, R. M.; Braden, D. J.; Dumesic, J. A., Dehydration of butanol to butene over solid acid catalysts in high water environments. J. Catal. 2009, 262(1), p. 134-143.

27. Higashio, Y.; Nakayama, T., One-step synthesis of methyl isobutyl ketone catalyzed by palladium supported on niobic acid. Catal. Today 1996, 28(1-2), p. 127-131.

28. (a) Pagán-Torres, Y. J.; Gallo, J. M. R.; Wang, D.; Pham, H. N.; Libera, J. A.; Marshall, C. L.; Elam, J. W.; Datye, A. K.; Dumesic, J. A., Synthesis of Highly Ordered Hydrothermally Stable Mesoporous Niobia Catalysts by Atomic Layer Deposition. ACS Catal. 2011, 1(10), p. 1234-1245; (b) Pham, H. N.; Pagan-Torres, Y. J.; Serrano-Ruiz, J. C.; Wang, D.; Dumesic, J. A.; Datye, A. K., Improved hydrothermal stability of niobia-supported Pd catalysts. Appl. Catal. A, 2011, 397(1-2), p. 153-162.

29. (a) Boffa, V.; ten Elshof, J. E.; Petukhov, A. V.; Blank, D. H., Microporous niobia-silica membrane with very low $\mathrm{CO} 2$ permeability. ChemSusChem 2008, 1(5), p. 437-443. (b) Boffa, V.; ten Elshof, J. E.; Garcia, R.; Blank, D. H. A., Microporous niobia-silica membranes: Influence of sol composition and structure on gas transport properties. Micropor. Mesopor. Mat. 2009, 118(1-3), p. 202-209;

30. Qi, H.; Han, J.; Xu, N.; Bouwmeester, H. J. M., Hybrid OrganicInorganic Microporous Membranes with High Hydrothermal Stability for the Separation of Carbon Dioxide. ChemSusChem 2010, 3(12), p. 1375-1378.

31. (a) Innocenzi, P.; Malfatti, L.; Kidchob, T.; Costacurta, S.; Falcaro, P.; Piccinini, M.; Marcelli, A.; Morini, P.; Sali, D.; Amenitsch, H., TimeResolved Simultaneous Detection of Structural and Chemical Changes during Self-Assembly of Mesostructured Films. J. Phys. Chem. C, 2007, 111(14), p. 5345-5350; (b) Malfatti, L.; Kidchob, T.; Falcaro, P.; Costacurta, S.; Piccinini, M.; Guidi, M. C.; Marcelli, A.; Corrias, A.; Casula, M. F.; Amenitsch, H.; Innocenzi, P., Highly ordered self-assembled mesostructured membranes: Porous structure and pore surface coverage. Micropor. Mesopor. Mat. 2007, 103(1-3), p. 113-122.

32. Stawski, T. M.; Veldhuis, S. A.; Besselink, R.; Castricum, H. L.; Portale, G.; Blank, D. H. A.; Ten Elshof, J. E., Nanostructure development in alkoxide-carboxylate-derived precursor films of barium titanate. J. Phys. Chem. C, 2012, 116(1), p. 425-434. 
33. Castricum, H. L.; Kreiter, R.; Van Veen, H. M.; Blank, D. H. A.; Vente, J. F.; Ten Elshof, J. E., High-performance hybrid pervaporation membranes with superior hydrothermal and acid stability. J. Membr. Sci. 2008, 324(1-2), p. 111-118.

34. Bras, W.; Dolbnya, I. P.; Detollenaere, D.; Van Tol, R.; Malfois, M.; Greaves, G. N.; Ryan, A. J.; Heeley, E., Recent experiments on a combined small-angle/wide-angle $X$-ray scattering beam line at the ESRF. J. Appl. Crystallogr. 2003, 36(3 I), p. 791-794.

35. Bras, W.; Emsley, J. W.; Levine, Y. K.; Luckhurst, G. R.; Seddon, J. M.; Timimi, B. A., Field-induced alignment of a smectic-a phase: A timeresolved x-ray investigation. J. Chem. Phys. 2004, 121(9), p. 43974413.

36. Guinier, A.; Fournet, G., Small-Angle Scattering of X-rays. John Wiley \& Sons: New York, 1955; Vol. 1, p 19-28, 40-52.

37. Feigin, L. A.; Svergun, D. U., Structure analysis by small angle X-ray and neutron scattering. Plenum Press: New York, 1987; $p$ 34-49, 5964, 68-73, 76-81, 84-94, 195-197, 230-239.

38. Porod, G., Small Angle X-ray Scattering. Acedamic Press: New York, 1982; Vol. 15, p 21-35, 41-48.

39. Teixeira, J., Small-angle scattering by fractal systems. J. Appl. Crystallogr. 1988, 21(6), p. 781-785;

40 Chen, S. H.; Teixeira, J., Structure and fractal dimension of proteindetergent complexes. Phys. Rev. Lett. 1986, 57(20), p. 2583-2586.

41. Boffa, V.; Castricum, H. L.; Garcia, R.; Schmuhl, R.; Petukhov, A. V.; Blank, D. H. A.; Ten Elshof, J. E., Structure and growth of polymeric niobia-silica mixed-oxide sols for microporous molecular sieving membranes: A saxs study. Chem. Mater. 2009, 21(9), p. 1822-1828.

42. Lin, M. Y.; Klein, R.; Lindsay, H. M.; Weitz, D. A.; Ball, R. C.; Meakin, $\mathrm{P} .$, The structure of fractal colloidal aggregates of finite extent. J. Colloid Interface Sci. 1990, 137(1), p. 263-280;

43. Sorensen, C. M.; Wang, G. M., Size distribution effect on the power law regime of the structure factor of fractal aggregates. Phys. Rev. E, 1999, 60(6 B), p. 7143-7148.

44. Kinning, D. J.; Thomas, E. L., Hard-sphere interactions between spherical domains in diblock copolymers. Macromol. 1984, 17(9), p. 1712-1718.

45. Terrill, N. J.; Crowley, T.; Gill, M.; Armes, S. P., Small-Angle X-ray Scattering Studies on Colloidal Dispersions of PolyanilineSilica Nanocomposites. Langmuir. 1993, 9, p. 2093-2096. 
46. (a) Schubert, K. V.; Strey, R. Kline, S. R.; Kaler, E. W., Small angle neutron scattering near Lifshitz lines: Transition from weakly structured mixtures to microemulsions J. Chem. Phys. 1994, 101(6), p. 5343-5355. (b) Teubner, M., Strey, R. Origin of scattering peaks in microemulsions. J. Chem. Phys. 1987, 87(5), p. 31953200.

47. (a) Stawski, T. M.; Veldhuis, S. A.; Besselink, R.; Castricum, H. L.; Portale, G.; Blank, D. H. A.; Ten Elshof, J. E., Nanoscale structure evolution in alkoxide-carboxylate sol-gel precursor solutions of barium titanate. J. Phys. Chem. C, 2011, 115(42),p. 20449-20459; (b) Stawski, T. M.; Veldhuis, S. A.; Besselink, R.; Castricum, H. L.; Portale, G.; Blank, D. H. A.; Ten Elshof, J. E., Erratum: Nanoscale structure evolution in alkoxide-carboxylate sol-gel precursor solutions of barium titanate (J. Phys. Chem. C (2010) 115 (2044920459) DOI: 10.1021/jp206572q). J. Phys. Chem. C 2011, 115(48), p. 24028.

48. Pedersen, J., Determination of size distribution from small-angle scattering data for systems with effective hard-sphere interactions. J. Appl. Crystallogr. 1994, 27(4), p. 595-608.

49. Flory, P. J., Molecular Size Distribution in Three Dimensional Polymers. III. Tetrafunctional Branching Units. J. Am. Chem. Soc. 1941, 63(11), p. 3096-3100.

50. Wagner, J., Small-angle scattering from spherical core-shell particles: an analytical scattering function for particles with SchulzFlory size distribution. J. Appl. Crystallogr. 2004, 37(5), p. 750-756.

51. Quintanilla, J.; Reidy, R. F.; Gorman, B. P.; Mueller, D. W., Gaussian random field models of aerogels. J. Appl. Phys. 2003, 93(8), p. 45844589.

52. Hjelm, R. P.; Schteingart, C.; Hofmann, A. F.; Sivia, D. S., Form and structure of self-assembling particles in monoolein-bile salt mixtures. J. Phys. Chem. 1995, 99(44), p. 16395-16406.

53. (a) Torma, V.; Peterlik, H.; Bauer, U.; Rupp, W.; Hüsing, N.; Bernstorff, S.; Steinhart, M.; Goerigk, G.; Schubert, U., Mixed silica titania materials prepared from a single-source sol-gel precursor: $A$ time-resolved SAXS study of the gelation, aging, supercritical drying, and calcination processes. Chem. Mater. 2005, 17(12), p. 31463153; (b) Sinkó, K.; Torma, V.; Kovács, A., SAXS investigation of porous nanostructures. J. Non-Cryst. Solids, 2008, 354(52-54), p. 5466-5474; (c) Freltoft, T.; Kjems, J. K.; Sinha, S. K., Power-law correlations and finite-size effects in silica particle aggregates 
studied by small-angle neutron scattering. Phys. Rev. B, 1986, 33(1), p. 269-275.

54. Svergun, D. I.; Koch, M. H. J., Small-angle scattering studies of biological macromolecules in solution. Rep. Prog. Phys. 2003, 66(10), p. 1735-1782.

55. Francisco, M. S. P.; Landers, R.; Gushikema, Y., Local order structure and surface acidity properties of a Nb205/SiO2 mixed oxide prepared by the sol-gel processing method J. Solid State Chem. 2004, 177,p. 2432-2439

56. (a) Avrami, M., Kinetics of phase change. I: General theory. J. Chem. Phys. 1939, 7(12), 1103-1112; (b) Avrami, M., Kinetics of phase change. II Transformation-time relations for random distribution of nuclei. J. Chem. Phys. 1940, 8(2), p. 212-224; (c) Avrami, M., Granulation, phase change, and microstructure kinetics of phase change. III. J. Chem. Phys. 1941, 9(2), p. 177-184.

57. Tobler, D. J.; Shaw, S.; Benning, L. G., Quantification of initial steps of nucleation and growth of silica nanoparticles: An in-situ SAXS and DLS study. Geochim. Cosmochim. Acta 2009, 73(18), p. 5377-5393.

58. Wondraczek, L.; Deubener, J.; Misture, S. T.; Knitter, R., Crystallization Kinetics of Lithium Orthosilicate Glasses. J. Am. Ceram. Soc. 2006, 89(4), p. 1342-1346;

59. Ferreira, E. B.; Lopez-Richard, V.; Zanotto, E. D.; Marques, G. E., Analytical Model for Heterogeneous Crystallization Kinetics of Spherical Glass Particles. J. Am. Ceram. Soc. 2009, 92(11), p. 26162618;

60. Pas, S. J.; Dargusch, M. S.; MacFarlane, D. R., Crystallisation kinetics of some archetypal ionic liquids: isothermal and non-isothermal determination of the Avrami exponent. Phys. Chem. Chem. Phys. 2011, 13 (25), p. 12033-12040.

61. Spendley, W.; Hext, G. R.; Himsworth, F. R., Sequential Application of Simplex Designs in Optimisation and Evolutionary Operation. Technometrics 1962, 4(4), p. 441-461.

62. Kessler, V. G.; Turova, N. Y.; Yanovskii, A. I.; Belokon, A. I.; Struchkov, Y. T., The structure of $\mathrm{Nb}_{8} \mathrm{O}_{10}(\mathrm{OEt})_{20}$ and the nature of crystalline metal alkoxides. Russ. J. Inorg. Chem. 1991, 36(7), p. 1-7. 


\section{S4.) Supporting Information Evolution of microstructure in mixed niobia-hybrid silica thin films from sol- gel precursors}




\section{S4.1. SAXS-measurements including the Porod regime}

For sample p60 two measurements were performed in the high $q$-regime $\left(3<q<32 \mathrm{~nm}^{-1}\right)$, one sol before drying and one powder that was dried for 44 min using an infra red lamp at a constant temperature $T=60^{\circ} \mathrm{C}$. The curves do not show an ideal $I \sim q^{-4}$ regime, but instead we observed correlation peaks with maxima at 14.6 and $17.1 \mathrm{~nm}^{-1}$ for $t_{\text {dry }}=0$ and $44 \mathrm{~min}$, respectively, which corresponded to separation distances of 0.43 and 0.37 $\mathrm{nm}$, respectively. The latter distance can be assigned to $\mathrm{Nb}-\mathrm{Nb}$ distances in amorphous $\mathrm{Nb}_{2} \mathrm{O}_{5}$ in the dried powder, while $\mathrm{Nb}-\mathrm{Si}$ distances were expected at lower distances of $0.34 \mathrm{~nm}$ [1]. However, since this correlation peak was rather broad we can neither confirm nor reject the presence of $\mathrm{Nb}-\mathrm{O}-\mathrm{Si}$ entities in this powder. The distances were larger in the sol before drying, indicating that it contained more loosely bound $\mathrm{Nb}-\mathrm{O}-\mathrm{Nb}, \mathrm{Nb}-\mathrm{O}-\mathrm{Si}$ and $\mathrm{Si}-\mathrm{O}-\mathrm{Si}$ entities. In any case, we were convinced that these correlation peaks should be assigned to atomic correlations that overlap with the Porod regime of small building blocks in the system.

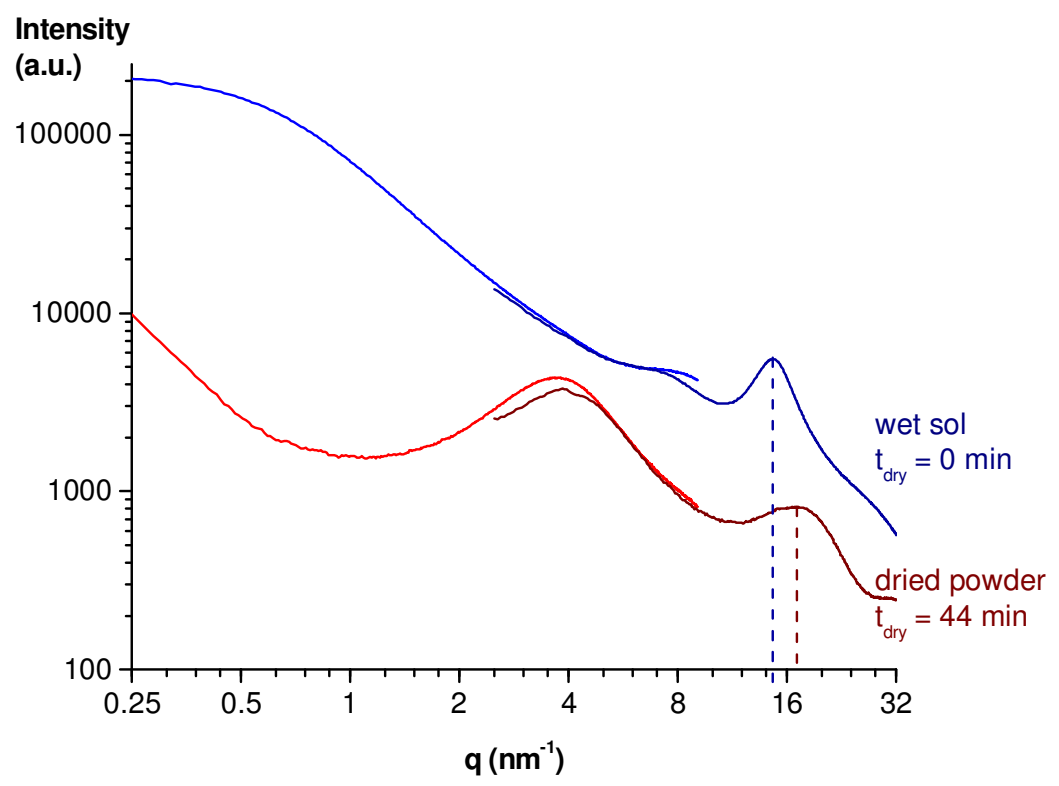

Figure S4.1. SAXS curves of sample $p 60$ at $t=0$ and $t=44 \mathrm{~min}$ during drying, measured in two different q-ranges: $0.25<q<9 \mathrm{~nm}^{-1}$ and $3<q<32 \mathrm{~nm}^{-1}$. 


\section{S4.2. Nitrogen sorption measurements.}

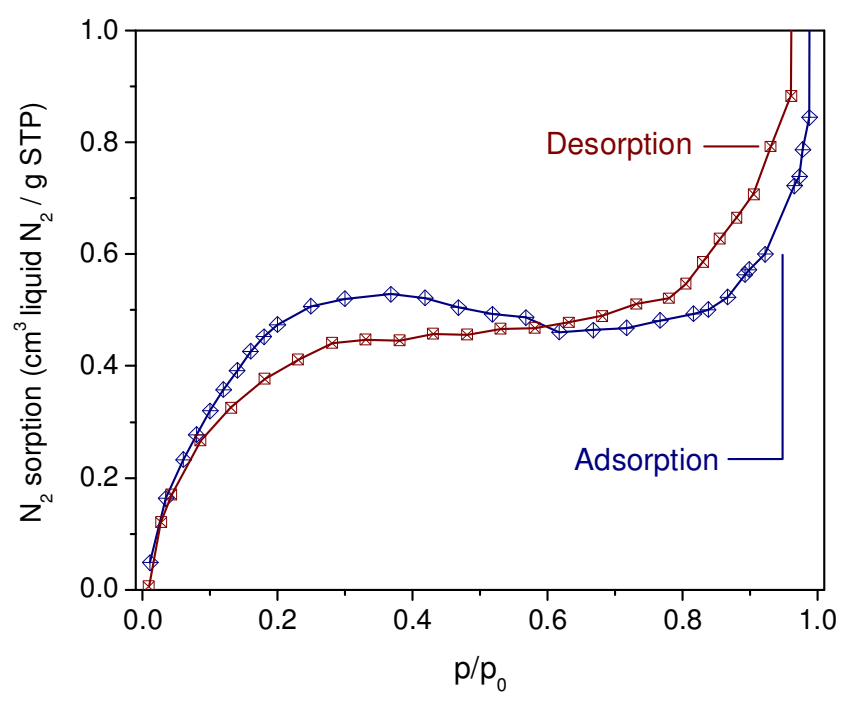

Figure S4.2. Nitrogen adsorption/desorption curve at $T=77 \mathrm{~K}$ of sample p60.

Nitrogen sorption measurements were performed at 77K in Micromeritics Tristar Instrument. The samples were firstly degassed at $300^{\circ} \mathrm{C}$ in a nitrogen atmosphere. From the adsorption isotherm, the surface area was determined by the Dubinin method, modified by Kaganer [2-3], represented by Equation (S4.1) with $n$ the gas adsorbed at relative pressure $p / p^{0}, n_{m}$ the monolayer capacity of the surface, both expressed in $\mathrm{mol} / \mathrm{g}$ adsorbent, and $D$ an adsorbate-dependent constant.

$\log (n)=\log \left(n_{m}\right)+D \cdot\left(\log \left(\frac{p^{0}}{p}\right)\right)^{2}$

Surface areas $A$ were subsequently determined according to Equation (S4.2) in which $N_{\mathrm{A}}$ is Avogadro's number and $a_{\mathrm{m}}$ the area occupied by a molecule in the completed monolayer, taken as $0.162 \mathrm{~nm}^{2}$ for $\mathrm{N}_{2}$ (ISO 9277).

$$
A=n_{\mathrm{m}} \cdot a_{\mathrm{m}} \cdot N_{\mathrm{A}}
$$


The nitrogen sorption measurements revealed a very low $\mathrm{N}_{2}$ sorption with a calculated surface area of $5,2 \mathrm{~m}^{2} / \mathrm{g}$. As we had observed for undoped BTESE powders, nitrogen sorption is low in these types of hybrid silicas compared to conventional $\mathrm{SiO}_{2}$ powders and membranes [4-5]. The microporosity can only be determined with gases that have a smaller kinetic diameter than nitrogen. Nevertheless, these results prove the absence of mesopores in the system.

\section{S4.3. References}

1. Francisco, M. S. P.; Landers, R.; Gushikema, Y., Local order structure and surface acidity properties of a Nb205/SiO2 mixed oxide prepared by the sol-gel processing method J. Solid State Chem. 2004, 177, p. 2432-2439

2. Dubinin, M. M. On physical feasibility of Brunauer's micropore analysis method, J. Colloid Interf. Sci. 1974, 46, p. 351-356

3. Kaganer, M. G. A new method for the determination of the specific adsorption surface of adsorbents and other finely dispersed substances, Zh. Fiz. Khim. 1959, 33, p. 2202-2210.

4. (a) Castricum, H. L.; Kreiter, R.; Van Veen, H. M.; Blank, D. H. A.; Vente, J. F.; Ten Elshof, J. E., High-performance hybrid pervaporation membranes with superior hydrothermal and acid stability. J. Membr. Sci. 2008, 324(1-2), p. 111-118. (b) Castricum, H. L.; Sah, A. Kreiter, R.; Blank, D. H. A.; Vente, J. F.; Elshof, J. E. ten Hydrothermally stable molecular separation membranes from organically linked silica, J. Mater. Chem. 2008, 18 p. 2150-2158.

5. Vos, R. M. de; Verweij, H. Improved performance of silica membranes for gas separation, J. Membr. Sci. 1998, 143, p. 37-51. 
Extent of niobia/silsesquioxane intermixing by solid state MAS-NMR 


\subsection{Abstract}

In this work different synthesis routes were evaluated with the aim of optimizing the incorporation of niobium within a hybrid silica matrix on an atomic scale. The fast kinetics of the hydrolysis/polycondensation of the organic $\mathrm{Nb}$ precursor $\mathrm{Nb}(\mathrm{OEt})_{5}$ entails a segregation of the resulting material into $\mathrm{Nb}_{2} \mathrm{O}_{5}$ and a silica based network. To overcome this effect we a) performed a prehydrolysis of 1,2 bis-triethoxy-ethane (BTESE) prior to adding niobium penta-ethoxide (NPE), or b) attempted to reduce the availability of $\mathrm{Nb}$ via a complexation of $\mathrm{Nb}$ by either acetyl acetone or 2methoxy-ethanol. The network organization was evaluated from results of FTIR as well as ${ }^{13} \mathrm{C},{ }^{29} \mathrm{Si}$ and ${ }^{17} \mathrm{O}$ MAS NMR spectroscopy. The prehydrolysis of BTESE and the addition of 2-methoxy-ethanol induced a but only moderate mixing of $\mathrm{Nb}$ and $\mathrm{Si}$, leading to a network in which islands of $\mathrm{Nb}_{2} \mathrm{O}_{5}$ are linked to the hosting silica based matrix via $\mathrm{Nb}-\mathrm{O}-\mathrm{Si}$ bonds. Contrary, the use of acetylacetonate lead to a mixing of $\mathrm{Nb}$ and $\mathrm{Si}$ on the atomic scale, producing a mixed $\mathrm{Nb}-\mathrm{O}-\mathrm{Si}$ network without any extended clusters of segregated $\mathrm{Nb}_{2} \mathrm{O}_{5}$. The $\mathrm{Si}-\mathrm{C}-\mathrm{C}-\mathrm{Si}$ bridge from the silsesquioxane is found to survive the condensation process and is even present in the resulting materials after annealing at $200^{\circ} \mathrm{C}$.

\subsection{Introduction}

Microporous hybrid silica membranes are known for both their chemical and thermal robustness [1-3]. Composite membranes with a selective hybrid silica layer have been successfully applied in pervaporation [1-5] of small molecules such as water and lower alcohols, and in gas separation [68]. The ability of microporous silica to separate such mixtures appears to be mainly based on size differences between molecules. We may extend the applicability of these membranes by incorporating transition metal oxides into the network to enhance the affinity for particular molecules. For instance, the introduction of nickel, cobalt, zirconium and niobium oxides in silica and hybrid silica membranes was found to enhance the $\mathrm{H}_{2} / \mathrm{N}_{2}$ and $\mathrm{H}_{2} / \mathrm{CO}_{2}$ permselectivity [9-15]. Yet, the homogeneous distribution of metal oxides into a bridged silsesquioxane matrix is not straightforward from a synthesis point of view: The metal oxide that is introduced, either as metal salt or as metal alkoxide, tends to phase separate while the silica matrix 
forms. In the case of metal alkoxides, phase separation is induced by the high reactivity of the metal alkoxide precursors that hydrolyze and condense much faster compared to silicon alkoxides. On the other hand, niobium oxalate was successfully used to incorporate niobia into the tetragonal environment of mesoporous MCM-41 silicates [16, 17]. However, the use of oxalates requires calcination temperatures above $500^{\circ} \mathrm{C}$, which makes them unsuitable for doping organically modified and hybrid silsesquioxanes.

The reactivity of metal alkoxides can be modified by introducing chelating ligands. Acetylacetone has often been applied to temper the reactivity of metal alkoxides. The enol tautomer of acetylacetone can dissociate one proton and subsequently form a bidentate complex with an enolic and ketonic oxygen atom [18]. The extent to which monodentate alcohol ligands are replaced by bidentate acetyl acetone ligands strongly depends on the type of metal ion. For instance, above an equimolar ratio of acetylacetone with respect to zirconium isopropoxide, zirconium readily forms an eightfold coordinated $\mathrm{Zr}(\mathrm{acac})_{4}$ complex with a complexation ratio (Hacac/M) of 4 [19]. On the other hand, adding acetylacetone to niobium (V) and titanium (IV) ethoxides only changes the oligomeric nature of these alkoxides from a dimeric and tetrameric form for niobium and titanium ethoxide, respectively $[20,21]$, towards monomeric complexes upon addition of sufficient acetylacetone [18, 22, 23]. Here, the metal centers keep their six-fold coordination, even when adding an excessive amount of either acetylacetone or 2-methoxy-ethanol [18]. It raises the question whether these chelating ligands substantially reduce the sensitivity of $\mathrm{Nb}(\mathrm{OEt})_{5}$ to such an extent that it enhances the homogeneous distribution of niobium centers within a hybrid silica matrix. ${ }^{17} \mathrm{O}-\mathrm{NMR}$ studies clearly revealed an increase of hetero linkages in zirconium and titaniumcontaining glasses when acetylacetone was added before sol formation, which makes this ligand a promising candidate for niobia doped glasses as well [24-26].

An alternative approach to temper the condensation rate of metal alkoxide precursors is to first hydrolyze the silicon ethoxide groups before adding the metal alkoxide. As a consequence, the free water concentration is reduced, while the concentration of hydrolyzed silica is higher. The latter species can potentially condense with niobium ethoxide moieties and form heterogeneous $\mathrm{Nb}-\mathrm{O}-\mathrm{Si}$ linkages. A previous SAXS study revealed that the size of electron-dense niobia clusters decreased when the bridged 
silsesquioxane precursor was firstly hydrolyzed for $1 \mathrm{~h}$ at $T=60^{\circ} \mathrm{C}$ [27]. Since $\mathrm{Nb}^{5+}$ is generally too large to fit into an oxygen anion tetrahedron, it will unlike silicon remain in an octahedral environment [27].

The current study is focused on the dispersion of $\mathrm{Nb}^{5+}$ cations inside a hybrid silsesquioxane matrix. The degree of mixing between $\mathrm{Nb}$ and $\mathrm{Si}$ is inferred from ${ }^{17} \mathrm{O}$ MAS NMR data. ${ }^{17} \mathrm{O}$ NMR has been shown to be a very powerful technique to gain insight in the dispersion and oxygen coordination of transition metal cations in silica based glasses [24-26, 28$32]$, since the isotropic chemical shift values for the ${ }^{17} \mathrm{O}$ nucleus in the various possible linkages ( $\mathrm{Si}-\mathrm{O}-\mathrm{Si}, \mathrm{Si}-\mathrm{O}-\mathrm{Nb}, \mathrm{Nb}-\mathrm{O}-\mathrm{Nb}$ ) are distinctively different.

\subsection{Experimental Section}

\subsubsection{Synthesis of mixed niobia/BTESE sols}

All precursor solutions were prepared inside a glovebox under dry nitrogen atmosphere. The reflux synthesis was performed under atmospheric conditions. For sol A the 1,2-bis-triethoxysilyl-ethane (abbreviated as BTESE, $A B C R$ chemicals, 97\%) precursor was initially partly hydrolyzed and condensed before adding niobium pentaethoxide (abbreviated as NPE, ABCR chemicals, 99.99\%) precursor. In a separate beaker NPE was diluted in ethanol yielding a molar concentration [NPE] $=0.54 \mathrm{~mol} / \mathrm{L}$. The BTESE sol was rapidly cooled down to room temperature in a cold water bath $\left(T=10^{\circ} \mathrm{C}\right)$ for $1 \mathrm{~min}$ and then the NPE solution was added dropwise. In the case of samples $B, C$ and D: NPE and BTESE were mixed in a molar ratio of $[\mathrm{BTESE}] /[\mathrm{NPE}]=2 / 1$. Then acetylacetone (acac) was added to sample $B$ $([\mathrm{acac}] /[\mathrm{Nb}]=2), 2$-methoxy-ethanol $(2 \mathrm{MOE})$ was added to sample $C([2 \mathrm{MOE}] /[\mathrm{Nb}]=2)$ and in the case of sample $D$ no additives were added. Then samples $B, C$ and $D$ were diluted in ethanol to yield a final overall ethoxide concentration of [-OEt] $=5.4 \mathrm{~mol} / \mathrm{L}$ (including silicon and niobium ethoxide groups). Then an acidic ethanol solution was added dropwise to samples $B, C$ and $D$ to yield the same hydrolysis and acid ratios as in sample A. Subsequently, sols $B, C$ and $D$ were refluxed for $1 \mathrm{~h}$ at $T=60^{\circ} \mathrm{C}$. All sols finally had an overall ethoxide concentration of $[-\mathrm{OEt}]=2.7 \mathrm{~mol} / \mathrm{L}$, a hydrolysis ratio $\left[\mathrm{H}_{2} \mathrm{O}\right] /[-\mathrm{OEt}]=1.0$, an acid ratio $\left[\mathrm{HNO}_{3}\right] /[-\mathrm{OEt}]=1 / 30$ and a niobium to silica ratio of $\mathrm{Nb} / \mathrm{Si}=1 / 4$. For the ${ }^{17} \mathrm{O}-\mathrm{NMR}$ experiments we hydrolyzed the sols with $35 \%{ }^{17} \mathrm{O}$-enrinched distilled water (Sigma-Aldrich) 
and for the FTIR experiments we hydrolyzed the sols with conventional doubly distilled water. The sols $(5 \mathrm{~mL})$ were dried for $30 \mathrm{~min}$ in a petridish (diameter $9 \mathrm{~cm}$ ) at $T=60^{\circ}$ Cusing a temperature controller coupled to a $150 \mathrm{~W}$ infrared lamp located $12 \mathrm{~cm}$ away from the sample. The dried film was ground and dried in a furnace at $T=60^{\circ} \mathrm{Cfor} 24 \mathrm{~h}$.

\subsubsection{FTIR-measurements}

The Fourier Transform Infrared (FTIR) measurements were performed using a Bruker Tensor 27 equipped with a $\mathrm{KBr}$ beamsplitter, a Pike Gladi ATR diamond attenuated total reflectance (ATR) unit and a liquid nitrogen cooled MCT broadband detector. The FTIR spectra were recorded in $5 \mathrm{~min}$, with a resolution of $1 \mathrm{~cm}^{-1}$.The Fourier transform (FT) was performed with 9480 phase interferogram points and a Blackman-Harris 3-term apodization function.

\subsubsection{Solid State NMR}

The ${ }^{13} \mathrm{C}$ CPMAS NMR experiments were performed employing a BRUKER Avance III spectrometer operating at resonance frequencies of $75.5 \mathrm{MHz}$ and $300.27 \mathrm{MHz}$ for ${ }^{13} \mathrm{C}$ and ${ }^{1} \mathrm{H}$, respectively. MAS was performed at 7000 $\mathrm{Hz}$ with a relaxation delay time of $10 \mathrm{~s}$. Typically, 1024 to 4096 scans were accumulated. The ${ }^{17} \mathrm{O}$ and ${ }^{29}$ Si MAS NMR spectra were recorded on a Varian VNMRs 500 spectrometer operating at $67.76 \mathrm{MHz}$ and $99.29 \mathrm{MHz}$ for ${ }^{17} \mathrm{O}$ and ${ }^{29} \mathrm{Si}$, respectively. The ${ }^{17} \mathrm{O}$ MAS NMR spectra were recorded at spinning speeds of $38 \mathrm{kHz}$ employing a Varian $1.6 \mathrm{~mm}$ triple resonance $\mathrm{T}^{3}$-MAS probe, using a recycle time of $1 \mathrm{~s}$. $\pi / 8$ pulses and single pulse excitation were used to acquire the data. The ${ }^{29} \mathrm{Si}$ MAS NMR spectra were recorded employing a Varian $5 \mathrm{~mm}$ triple resonance $\mathrm{T}^{3}$ MAS probe, a MAS frequency of $7 \mathrm{kHz}$ and a recycle delay of $150 \mathrm{~s}$. Spectra are referenced to TMS for ${ }^{29} \mathrm{Si}$, Adamantane (downfield signal at $38.5 \mathrm{ppm}$ ) for ${ }^{13} \mathrm{C}$, and $\mathrm{H}_{2}{ }^{17} \mathrm{O}$ for ${ }^{17} \mathrm{O}$. 


\subsection{Results and discussion}

The FTIR spectra for the as prepared samples are collected in Figure 5.1 together with the spectrum of anhydrous, pristine ethanol for comparison. The signals are dominated by peaks that are associated with ethanol and hydrolysed and condensed silicon moeities. Only in sample B, which containes acetylacetone, we observed a small shoulder at $940 \mathrm{~cm}^{-1}$. This value is close to $960 \mathrm{~cm}^{-1}$ which is often associated with Si-O-Nb moieties $[33,34]$. However, since this vibration is detected after addition of acetylacetone to NPE, we assign this signal to vibrations of conjugated $\mathrm{C}=\mathrm{O}$ and $\mathrm{C}-\mathrm{C}$ bonds of the acetylacetone ligand coordinated to niobium [35].

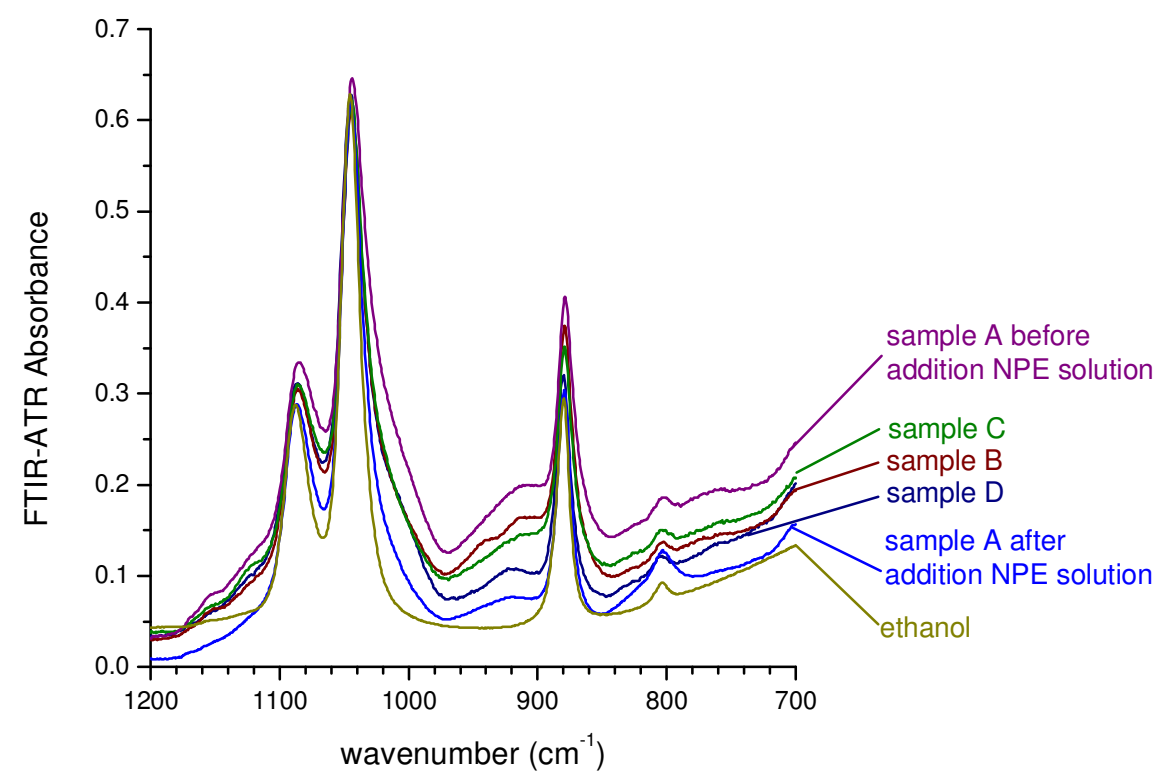

Figure 5.1. FTIR spectra of sols A-D (before drying).

Vibrations at $1150 \mathrm{~cm}^{-1}$ and $760 \mathrm{~cm}^{-1}$ indicate the presence of remnant SiOEt groups of the BTESE precursor [36]. These vibrations are substantially broadener than in pure BTESE, most likely due to the polar character of the surrounding medium. This made it difficult to quantify the amount and 
compare the different samples. The Si-OEt vibration at $760 \mathrm{~cm}^{-1}$ was the weakest for sample $B$, which indicates that for the acac sample almost all ethoxide groups were released from silicon.

The addition of NPE to the prehydrolyzed BTESE (sample A) exhibits a clear effect on the FTIR spectrum. The intensity of the vibrations at $920 \mathrm{~cm}^{-1}$ and $1020 \mathrm{~cm}^{-1}$ that are assigned to $\mathrm{Si}-\mathrm{OH}$ and $\mathrm{Si}-\mathrm{O}-\mathrm{Si}$ vibrations, respectively [36], is considerably smaller after addition of NPE. Such a reduction cannot be explained solely by the effect of dilution (1.4 times on volume). Possibly, the addition of NPE reduced the free water concentration and since the free water concentration is coupled with the concentration of hydrolyzed and condensed silicon species, their concentrations reduced as well. Thus, the addition of NPE may have reversed the prehydrolysis proces of BTESE, i.e. an esteriffication proces with the formation of SiOEt. However, the vibration at $1150 \mathrm{~cm}^{-1}$ and $760 \mathrm{~cm}^{-1}$ associated with SiOEt did not increase upon NPE addition and a vibration around $800 \mathrm{~cm}^{-1}$ increased instead. The excact cause of the increased intensity around $800 \mathrm{~cm}^{-1}$ remained unclear.

It is noted that NPE also has vibrations located around $920 \mathrm{~cm}^{-1}$ [37]. Nevertheless, in a separate experiment in the absence of BTESE we observed that this vibration peak rapidly reduced when an equivalent amount of water was added. Therefore, the peak at $920 \mathrm{~cm}^{-1}$ is probably dominated by $\mathrm{Si}-\mathrm{OH}$ vibrations instead of Nb-O-Et vibrations.

Therefore, it is questionable whether prehydrolysis of silica had a substantial effect on the distribution of niobia in the sol. Upon comparison of samples B and C, which contained acetylacetone and 2-methoxyethanol as chelating ligands, respectively, with sample $D$ that had no chelating additives, an enlarged Si-OH vibration was observed at $920 \mathrm{~cm}^{-1}$ in the latter sample. For both samples $B$ and $C$, the presence of chelating ligands may have reduced the amount of water that reacted with the NPE precursor, so that more water was available for hydrolysis of BTESE.

The ${ }^{13} \mathrm{C}$ CPMAS and ${ }^{29}$ Si MAS NMR spectra of the ${ }^{17} \mathrm{O}$-enriched sols, dried at $T=60^{\circ} \mathrm{C}$, are shown in Figure. 5.2. In the ${ }^{13} \mathrm{C}$ CPMAS NMR spectra (Figure 5.2a), the signal at approx. $4 \mathrm{ppm}$ may be assigned to the ethylene bridge carbon of BTESE. Its presence confirms that the Si-C-C-Si bridge stays intact during the hydrolysis and polycondensation steps. The weak signals around $18 \mathrm{ppm}$ and $58 \mathrm{ppm}$ may be assigned to the methyl and methylene group of ethanol/ethoxy groups. This indicates either the presence of ethanol, presumably trapped within the pores of the membrane, or an incomplete 
hydrolysis/polycondensaton of the mixed network. In the spectrum of sample C, we find additional signals at approx. $61.8 \mathrm{ppm}$ and $74.0 \mathrm{ppm}$, which can be assigned to the $C_{\alpha}$ and $C_{\beta}$ carbon nuclei of the 2methoxyethanol (as represented by $\mathrm{HO}-\mathrm{C}_{\alpha} \mathrm{H}_{2}-\mathrm{C}_{\beta} \mathrm{H}_{2}-\mathrm{O}-\mathrm{C}_{\gamma} \mathrm{H}_{3}$ ) added as complexing agent. Likewise, in the spectrum of $D$, the additional signals at $27 \mathrm{ppm}$ ( $\mathrm{C}_{\alpha}$ of acetylacetone), $106 \mathrm{ppm}$ ( $C_{\beta}$ of acetylacetone) and $193 \mathrm{ppm}$ ( $C_{\gamma}$ of acetylacetone), indicate the presence of immobilized acetylacetonate ligands $\left(\left[\mathrm{H}_{3} \mathrm{C}_{\alpha}-\left(\mathrm{C}_{\beta} \mathrm{O}\right)-\mathrm{C}_{\gamma} \mathrm{H}=\left(\mathrm{C}_{\beta} \mathrm{O}\right)-\mathrm{C}_{\alpha} \mathrm{H}_{3}\right]^{-}\right)$. Since the signal for $\mathrm{C}_{\beta}$ was found at $106 \mathrm{ppm}$ and no extended intensity was observed in the range of 58-60 $\mathrm{ppm}$, the overwhelming majority of the acetylacetone ligand is present in its enolic form.
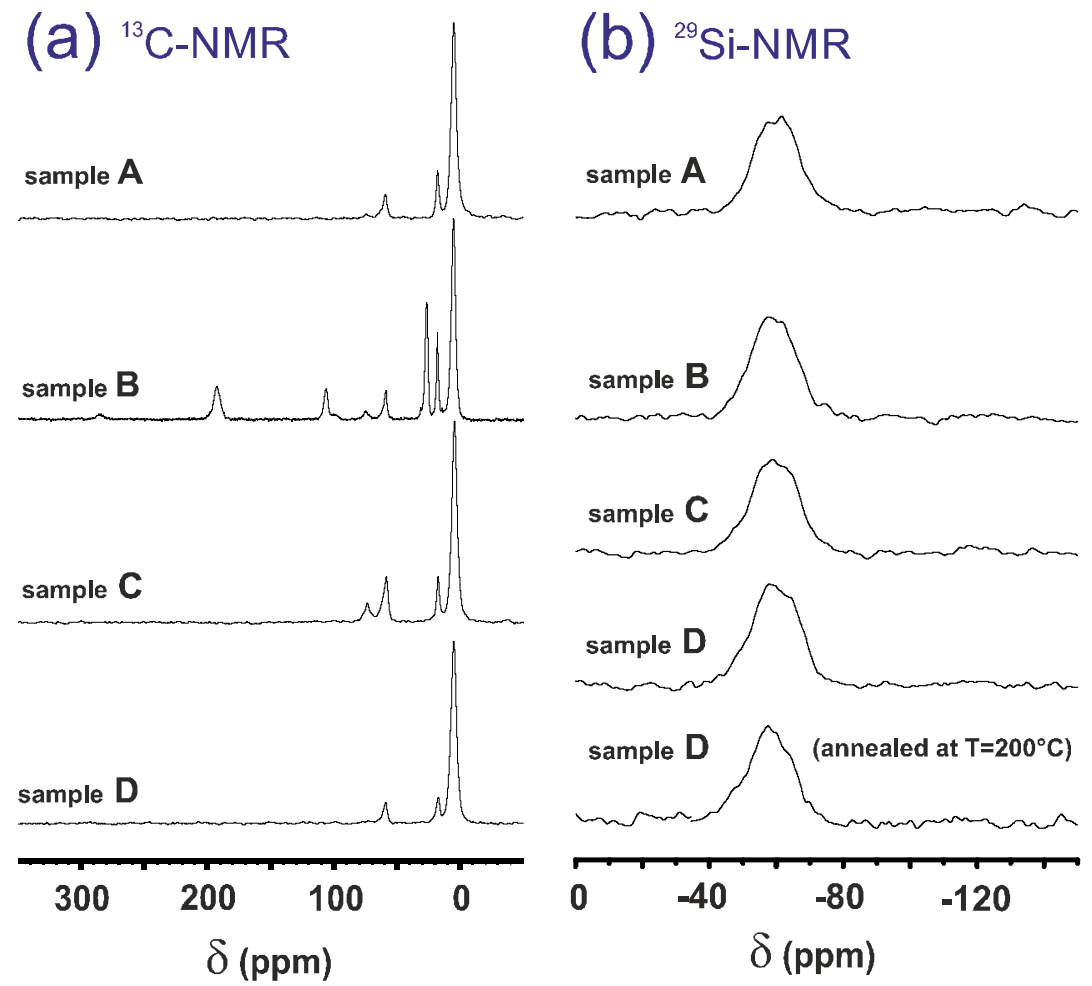

Figure 5.2. ${ }^{13} \mathrm{C}$ CPMAS NMR spectra (a) and ${ }^{29}$ Si MAS NMR spectra (b) for the solgel derived niobia-hybris silica powders studied in this work. Spinning speed of 6 $\mathrm{kHz}$ were used to record the spectra. For the ${ }^{13} \mathrm{C}$ CPMAS spectra, ${ }^{13} \mathrm{C}$ and ${ }^{1} \mathrm{H}$ rf fields of $50 \mathrm{kHz}$, a contact time of 2 msand a repetition delay of $5 \mathrm{~s}$ were used for the Hartmann-Hahn matching. 
Since the magnetization transfer from ${ }^{1} \mathrm{H}$ to ${ }^{13} \mathrm{C}$ in the cross polarization (CP) process relies on the magnetic dipole coupling between these nuclei, any dynamic process, averaging this dipole interaction, will reduce the corresponding signal intensity. Therefore, the CP spectra are intrinsically non-quantitative, all signals from dynamic species (e.g. free ethanol within the pores) is essentially filtered out. Thus, the spectra confirm the formation of immobilized 2-methoxyethanol and acetylacetone species, presumably connected to $\mathrm{Nb}$. The ${ }^{29} \mathrm{Si}$ MAS NMR spectra for all samples (Figure 5.2b) reveal several resonances in a range between -50 and -70 $\mathrm{ppm}$. These could not completely be resolved due to the strong overlap between the signals. Based on literature data, this is the region of $T_{2}$ (C$\left.\mathrm{Si}(\mathrm{OSi})_{2} \mathrm{OH},-56 \mathrm{ppm}\right)$ and $\mathrm{T}_{3}$ species $\left(\mathrm{C}-\mathrm{Si}(\mathrm{OSi})_{3},-66 \mathrm{ppm}\right)$. In accordance with the ${ }^{13} \mathrm{C}$ data, this confirms the survival of the ethylene bridge during the hydrolysis/condensation steps. From an analysis of the spectra, the degree of condensation was estimated ( $0 \%$ for T0, 100\% for T3). Since a deconvolution of the spectra was not possible in all cases, we determined the center of gravity (CG) for all spectra, which was found to be in the range of $-59 \mathrm{ppm}$ to $-60 \mathrm{ppm}$ for all samples (Table 5.1). The resulting condensation degree in $\%-67+\left(\delta_{\mathrm{CG}}-\delta_{\mathrm{T} 2}\right) \cdot 33 /\left(\delta_{\mathrm{T}_{3}}-\delta_{\mathrm{T} 2}\right)$ is found in the range of $75 \%$, independent on the synthesis method. Fig. $2 \mathrm{~b}$ shows the ${ }^{29} \mathrm{Si}$ MAS NMR spectrum of sample $D$, annealed at $200{ }^{\circ} \mathrm{C}$. The spectrum was, within the experimental error of these experiments, found to have similar resonances as the corresponding spectrum of the non-annealed sample. No resonance frequencies in the range of $-90 \mathrm{ppm}$ to $-120 \mathrm{ppm}$ were observed, which indicated the absence of $\mathrm{SiO}_{4}$-species. From this we can conclude that the Si-C-C-Si bridge even survives annealing at $200^{\circ} \mathrm{C}$.

While the ${ }^{13} \mathrm{C}$ and ${ }^{29} \mathrm{Si}$ MAS NMR spectra offer some information about the general hydrolysis and condensation of the network, no information is revealed concerning the degree of mixing between $\mathrm{Nb}$ and $\mathrm{Si}$. Information about this mixing may be deduced from an estimation of the magnetic dipole coupling between ${ }^{29} \mathrm{Si}$ and ${ }^{93} \mathrm{Nb}$ from the results of ${ }^{29} \mathrm{Si}^{93} \mathrm{Nb}$ REAPDOR NMR experiment. However, a more direct approach involves the measurement of the ${ }^{17} \mathrm{O}$-MAS NMR spectra at very high spinning speeds. This is possible since the isotropic chemical shift values for the possible ${ }^{17} \mathrm{O}$ signals, $\mathrm{Si}-\mathrm{O}-\mathrm{Si}, \mathrm{Si}-\mathrm{O}-\mathrm{Nb}, \mathrm{ONb}_{2}$ and $\mathrm{ONb}_{3}$ are distinctively different. Whereas oxygen bridging two $\mathrm{SiO}_{4}$ is found at a chemical shift value around $65 \mathrm{ppm}$, the ${ }^{17} \mathrm{O}$ signals in sol-gel processed $\mathrm{Nb}_{2} \mathrm{O}_{5}$ are reported at $360 \mathrm{ppm}\left(\mathrm{ONb}_{3}\right)$ and $560 \mathrm{ppm}\left(\mathrm{ONb}_{2}\right)$. In a study on mixed $\mathrm{Nb}_{2} \mathrm{O}_{5}-\mathrm{SiO}_{2}$ glasses, Drake et al. [29] assigned the signal at $250 \mathrm{ppm}$ to $\mathrm{Nb}-\mathrm{O}-\mathrm{Si}$ linkages. 


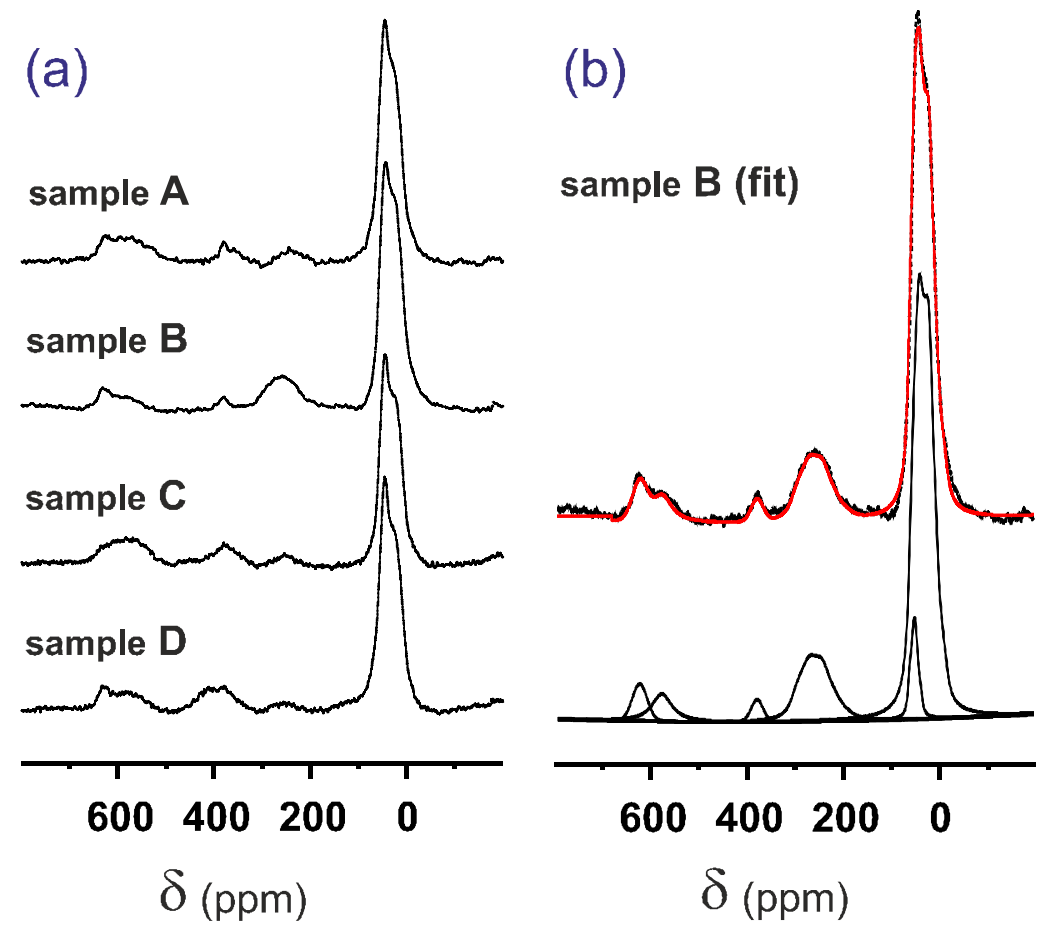

Figure 5.3. a) single pulse ${ }^{17} \mathrm{O}-\mathrm{MAS}$-NMR spectra of ${ }^{17} \mathrm{O}$-enriched sol-gel derived niobia-hybrid silica powders, samples A-D. The spectra were recorded at $38 \mathrm{kHz}$, emplyoying $\pi / 8$ pulses and a repetition delay of $1 \mathrm{~s}$. The signal at $626 \mathrm{ppm}$ represents a spinning sideband of the Si-O-Si signal at $65 \mathrm{ppm}$. b) ${ }^{17} \mathrm{O}$ MAS NMR spectrum of B together with its deconvolution.

The ${ }^{17} \mathrm{O}$ MAS NMR spectra for the studied samples are shown in Figure 5.3a. The single pulse excitation spectra were recorded using an excitation pulse of width $<\pi / 8$ to ensure quantitative excitation. Attempts to detect the spectra employing a spin echo sequence were abandoned since the downfield signals ( $\mathrm{Nb}-\mathrm{O}-\mathrm{Si}, \mathrm{ONb}_{2}$ and $\mathrm{ONb}_{3}$ ) exhibited a considerably reduced intensity as compared to the single pulse excitation spectrum, presumably due to short $T_{2}$ * values. While the spectra for samples $A, C$ and D exhibit all four signals (Si-O-Si at 65 ppm, Si-O-Nb at 300 ppm, $\mathrm{ONb}_{3}$ at 
$350 \mathrm{ppm}$ and $\mathrm{ONb}_{2}$ at $560 \mathrm{ppm}$ ), in the spectrum for sample $\mathrm{B}$ only the signals for $\mathrm{Si}-\mathrm{O}-\mathrm{Si}, \mathrm{Nb}-\mathrm{O}-\mathrm{Si}$ and $\mathrm{ONb}_{2}$ can be identified. For the simulation of the ${ }^{17} \mathrm{O}$ signal assigned to oxygen in Si-O-Si linkages (65 ppm) we had to use a set of two second order quadrupolar patterns. The dominating contribution exhibits a quadrupolar coupling constant $\mathrm{C}_{\mathrm{Q}}=4.8 \mathrm{MHz}$ and an asymmetry parameter $\eta_{\mathrm{Q}}=0$ for all samples, and isotropic chemical shift values of $62-64 \mathrm{ppm}$, in perfect agreement with published data. The minor signal shows quadrupolar parameters of $\mathrm{C}_{\mathrm{Q}}=5.3-5.8 \mathrm{MHz}, \eta_{\mathrm{Q}}=0.9$ and an isotropic chemical shift of $66-72 \mathrm{ppm}$. This second signal may be assigned to terminal $\mathrm{Si}-\mathrm{OH}$ groups, the presence of which is suggested by the results of the ${ }^{29} \mathrm{Si}$ MAS NMR spectra (vide supra). For the signal assigned to Nb-O-Si bridges the simulation produces quadrupolar parameters $\mathrm{C}_{\mathrm{Q}}=5.3-5.7$ $\mathrm{MHz}, \eta_{\mathrm{Q}}=0.5$ and chemical shift values of $280-310 \mathrm{ppm}$. The remaining signals $\left(\mathrm{ONb}_{2}\right.$ and $\left.\mathrm{ONb}_{3}\right)$ did not have a well-defined shape. Therefore, Gaussian functions were used to simulate the signals of these entities, which can only provide the center of gravity and relative area of these lines. Fig. $3 \mathrm{~b}$ exemplarily shows the simulation for the ${ }^{17} \mathrm{O}$ MAS NMR spectrum of sample B. The results of a simulation of the spectra are collected in Table 1.

Before analyzing the results, let us first briefly discuss the expected ${ }^{17} \mathrm{O}$ spectra for the two limiting cases of a) a complete segregation into a silicabased network and a $\mathrm{Nb}_{2} \mathrm{O}_{5}$ network and $\mathrm{b}$ ) a homogeneous distribution of $\mathrm{Nb}$ and $\mathrm{Si}$, both for the given $\mathrm{Si} / \mathrm{Nb}$ ratio of $4: 1$. For the former, we would expect $71 \%$ of the oxygen to be incorporated in Si-O-Si bridges and $29 \%$ of the oxygen to be involved in $\mathrm{Nb}-\mathrm{O}-\mathrm{Nb}$ bonding. For a homogeneous distribution on the other hand we can calculate the relative ratios for the various types of $\mathrm{A}-\mathrm{O}-\mathrm{B}$ bridges with $\mathrm{A}, \mathrm{B}=\mathrm{Si}, \mathrm{Nb}$ as follows. For $\mathrm{a}$ composition $4 \cdot\left(\mathrm{O}_{3} \mathrm{Si}-\mathrm{C}-\mathrm{C}-\mathrm{SiO}_{3}\right): 1 \mathrm{Nb}_{2} \mathrm{O}_{5}$, ignoring oxygen triclusters, we have 34 bonds to oxygen, 24 from $\mathrm{Si}$ and 10 from $\mathrm{Nb}$. This results in probabilities of $(24 / 34)^{2}=0.5$ for Si-O-Si, $24 / 34 \cdot 10 / 34 \cdot 2=0.4$ for Si-O-Nb and $(10 / 34)^{2}$ $=0.1$ for $\mathrm{Nb}-\mathrm{O}-\mathrm{Nb}$. 
Table 1: results of the simulation of the ${ }^{17} \mathrm{O}$ and ${ }^{29} \mathrm{Si}$ MAS NMR single pulse excitation spectra of the measured samples. The ${ }^{17} \mathrm{O}$ NMR signals for Si-O-Si and $\mathrm{Nb}-\mathrm{O}-\mathrm{Si}$ were simulated assuming a second order quadrupolar line shape. Due to the lack of resolution, the signals for $\mathrm{O}_{3} \mathrm{Nb}$ and $\mathrm{O}_{2} \mathrm{Nb}$ were simulated using Gaussion functions. For the ${ }^{29} \mathrm{Si}$ MAS NMR spectra, the center of gravity for the signals is given.

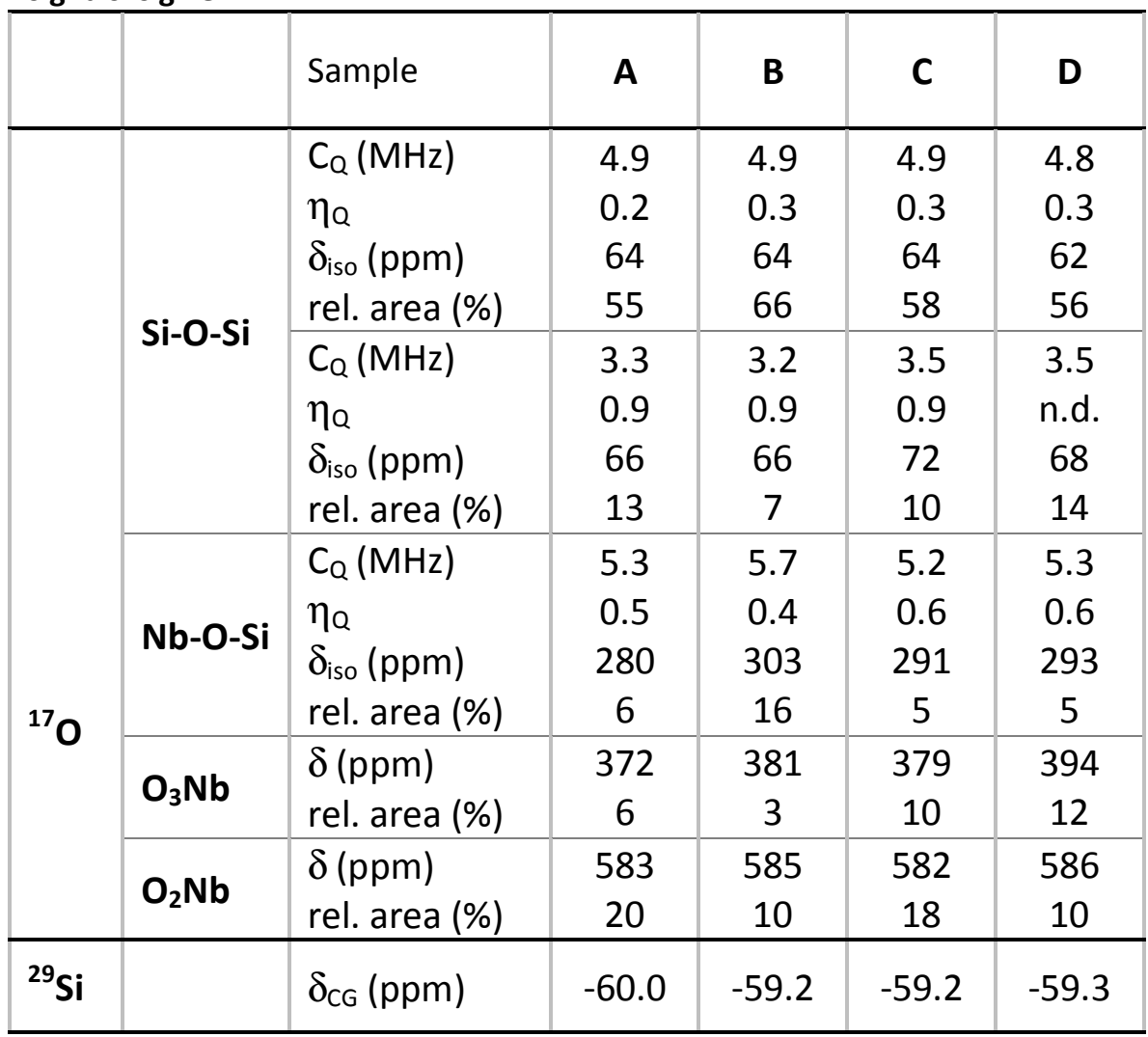

Based on the ${ }^{13} \mathrm{C}$ NMR data a distinct amount of acetylacetonate (sample B) and 2-methoxy-ethanol (sample $\mathrm{C}$ ) is still present in the samples. This affects the total number of possible $\mathrm{Nb}-\mathrm{O}-\mathrm{Si}$ and $\mathrm{Nb}-\mathrm{O}-\mathrm{Nb}$ in the above calculation. As a consequence, no quantitative evaluation of the network adopted is possible and we can only take the relative fraction of $\mathrm{Nb}-\mathrm{O}-\mathrm{Si}$ as a rough measure for the degree of mixing in the samples studied. 
Two main observations may be drawn from the results presented in Figure 5.3 and Table 5.1. Firstly, all samples exhibit - to a given extent - a mixing between $\mathrm{Nb}$ and $\mathrm{Si}$ on the atomic scale, as evidenced by the existence of the resonance at $300 \mathrm{ppm}$ which originates in oxygen involved in $\mathrm{Nb}-\mathrm{O}-\mathrm{Si}$ linkages. Secondly, the extent of mixing between $\mathrm{Nb}$ and $\mathrm{Si}$, proves to be rather limited, with the exception of sample $B$, which was prepared employing acetylacetone as the complexing agent. Thus in samples $A, C$, and $\mathrm{D}$, our results indicated a segregation of the networks in a Nb-rich and a silica rich part. These $\mathrm{Nb}_{2} \mathrm{O}_{5}$ segregations contain $\mathrm{O}-\mathrm{Nb}_{3}$ and $\mathrm{O}-\mathrm{Nb}_{2}$ which produce the signals around $380 \mathrm{ppm}$ and $585 \mathrm{pppm}$. These values are rather close to those of the corresponding signals in a sol-gel derived $\mathrm{Nb}_{2} \mathrm{O}_{5}$ (550 ppm and $360 \mathrm{ppm}$ ) [30, 34].

The network in these samples can be described by a silsesquioxane-based network with segregated $\mathrm{Nb}_{2} \mathrm{O}_{5}$ particles distributed within the matrix. Only some $\mathrm{Nb}-\mathrm{O}-\mathrm{Si}$ bridges link these $\mathrm{Nb}_{2} \mathrm{O}_{5}$ segregates to the silica network. Contrary to this, in sample $\mathrm{B}$ the degree of mixing seems to be much more pronounced. Here, the observed relative areas of the individual ${ }^{17} \mathrm{O}$ signals are much closer to a homogeneous mixture of $\mathrm{Nb}$ and $\mathrm{Si}$. Moreover, in this case, no $\mathrm{ONb}_{3}$ units are observed. Clearly, addition of acetylacetone succeeds in slowing down the hydrolysis and polycondensation of $\mathrm{Nb}(\mathrm{OEt})_{5}$, prevents larger aggregates of $\mathrm{Nb}_{2} \mathrm{O}_{5}$ being formed and consequently increases the relative amount of $\mathrm{Si}-\mathrm{O}-\mathrm{Nb}$ bridges.

A network model in accordance with all the observed data is presented in Figure 4. Small regions rich in $\mathrm{Nb}-\mathrm{O}-\mathrm{Nb}$ bonding are embedded within a silsesquioxane-based matrix, both connected via $\mathrm{Nb}-\mathrm{O}-\mathrm{Si}$ linkages. For samples $A, C$ and $D$, these regions are relatively large and are more adequately described by nanoscaled $\mathrm{Nb}_{2} \mathrm{O}_{5}$ clusters. For sample $\mathrm{B}$, these regions are considerably smaller, in addition, only a rather small amount of $\mathrm{ONb}_{3}$ units seems to be present. The fraction of $\mathrm{Nb}-\mathrm{O}-\mathrm{Si}$ linkages found in this sample is $40-70 \%$ of the value expected for a statistical mixing of $\mathrm{Nb}$ and $\mathrm{Si}$ (depending on the amount of acac complexing $\mathrm{Nb}$ in the final material). The ethylene bridge is still present in the final network. Even at an annealing temperature of $200{ }^{\circ} \mathrm{C}$, the bonds between $\mathrm{Si}$ and $\mathrm{C}$ do not suffer from being cleaved, as illustrated by the ${ }^{13} \mathrm{C}$ and ${ }^{29} \mathrm{Si}$ MAS NMR spectra of sample D, annealed at $200{ }^{\circ} \mathrm{C}$ (data not shown). This result supports previously reported TGA-MS and FTIR measurements, which suggested that methylene bridged carbon atoms remained present in BTESE-based hybrids up to $T=300^{\circ} \mathrm{C}$ [38]. Yet, more specifically the survival 
of the Si-C bond upon annealing was unambiguously proved by these ${ }^{13} \mathrm{C}$ and ${ }^{29} \mathrm{Si}$ MAS NMR results.

\subsection{Conclusions}

Among the different approaches that were applied to enhance the dispersion of sol-gel derived niobia within the silsesquioxane matrix, the use of acetylacetone as a chelating ligand was found to be the most effective approach. The acac complex partly blocks the hydrolysis of niobium ethoxide. This entails an enhanced degree of hydrolyzed BTESE, as suggested by the enlarged intensity of the $\mathrm{Si}-\mathrm{OH}$ stretch vibration in the FTIR experiment. The ${ }^{17} \mathrm{O}$ data indicate an increased chance that a hydrolyzed silicon crosslinks with a niobium species.

The distribution of oxygen among $\mathrm{Si}-\mathrm{O}-\mathrm{Si}, \mathrm{Nb}-\mathrm{O}-\mathrm{Si}$ and $\mathrm{Nb}-\mathrm{O}-\mathrm{Nb}$ is found to be rather close to a statistical distribution, indicating an effective mixing of $\mathrm{Nb}$ and $\mathrm{Si}$. Addition of 2-methoxyethanol on the other hand does not entail a substantial effect on the hydrolysis/polycondensation kinetics and subsequently on the network being formed. Contrary to the case of the acac ligand, only a fraction of $\mathrm{Nb}^{5+}$ will be coordinated by 2-MOE unless all the ethanol is removed by evaporation, as reported earlier. Therefore, its stabilizing effect is limited. As a consequence, the reactivity of NPE is only slightly reduced, in accordance with our observations. 


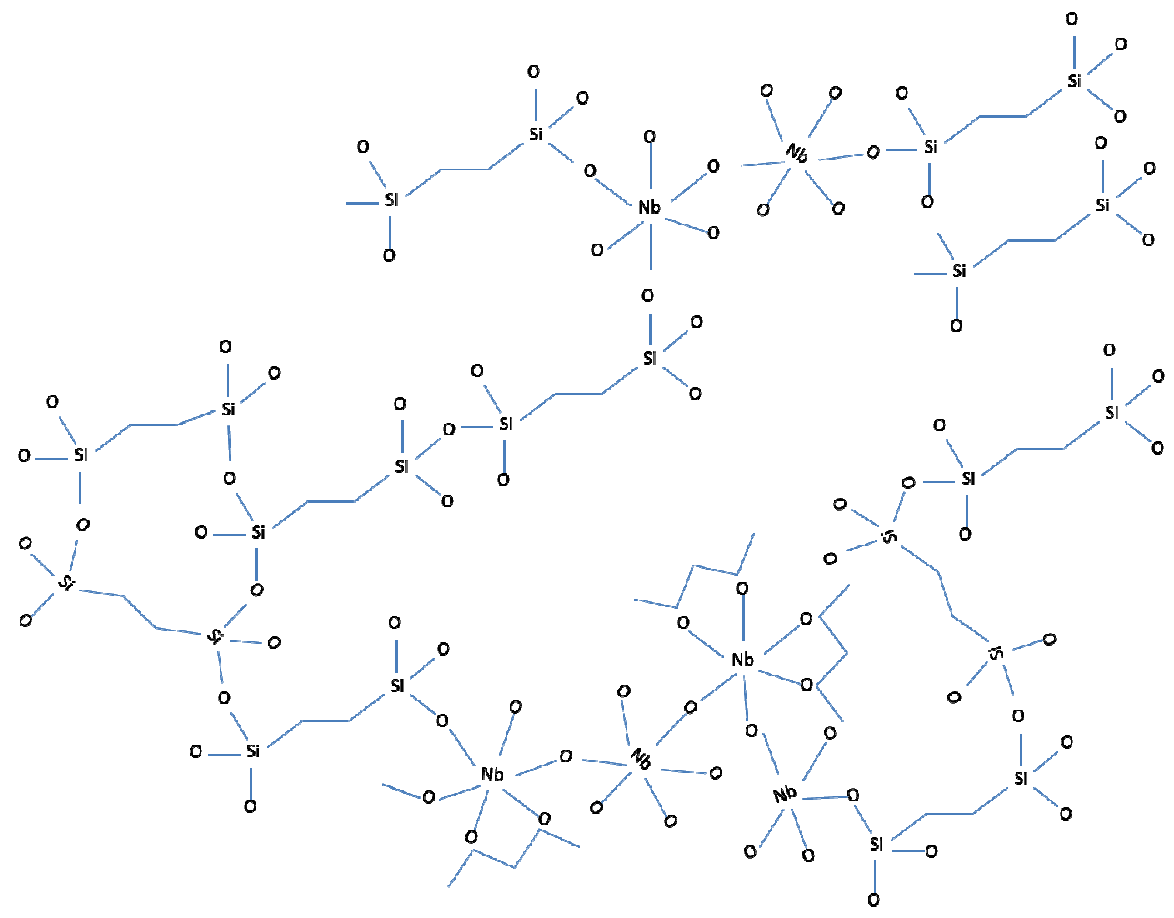

Figure 5.4. Sketch that illustrates the network organization in sample B as deduced from the NMR results.

\subsection{References}

1. Castricum, H.L., et al., High-performance hybrid pervaporation membranes with superior hydrothermal and acid stability. J. Membr. Sci., 2008. 324(1-2): p. 111-118.

2. Kong, C., et al., Enhanced performance of inorganic-polyamide nanocomposite membranes prepared by metal-alkoxide-assisted interfacial polymerization. J. Membr. Sci., 2011. 366(1-2): p. 382388.

3. Castricum, H.L., et al., Hybrid ceramic nanosieves: Stabilizing nanopores with organic links. Chem. Commun., 2008 (9): p. 11031105.

4. Kreiter, R., et al., Stable hybrid silica nanosieve membranes for the dehydration of lower alcohols. ChemSusChem, 2009. 2(2): p. 158160. 
5. Agirre, I., et al., Hybrid organosilica membranes and processes: Status and outlook. Sep. Purif. Technol., 2014. 121: p. 2-12.

6. Kanezashi, M., et al., Organic-inorganic hybrid silica membranes with controlled silica network size: Preparation and gas permeation characteristics. J. Membr. Sci., 2010. 34 (1-2): p. 310-318.

7. Kreiter, R., et al., Evaluation of hybrid silica sols for stable microporous membranes using high-throughput screening. J. SolGel Sci. Technol., 2011. 57(3): p. 245-252.

8. Chang, K.-S., et al., A molecular dynamics simulation of a homogeneous organic-inorganic hybrid silica membrane. Chem. Commun., 2010. 46(48): p. 9140-9142.

9. Igi, R., et al., Characterization of co-doped silica for improved hydrothermal stability and application to hydrogen separation membranes at high temperatures. J. Am. Ceram. Soc., 2008. 91(9): p. 2975-2981.

10. Kanezashi, M. and M. Asaeda, Hydrogen permeation characteristics and stability of Ni-doped silica membranes in steam at high temperature. J. Membr. Sci., 2006. 271(1-2): p. 86-93.

11. $\mathrm{Qi}, \mathrm{H}$., et al., Effect of $\mathrm{Nb}$ content on hydrothermal stability of a novel ethylene-bridged silsesquioxane molecular sieving membrane for H2/CO2 separation. J. Membr. Sci., 2012. 421-422: p. 190-200.

12. Qi, H., et al., Hybrid Organic-Inorganic Microporous Membranes with High Hydrothermal Stability for the Separation of Carbon Dioxide. ChemSusChem, 2010. 3(12): p. 1375-1378.

13. Boffa, V., et al., Microporous niobia-silica membrane with very low CO2 permeability. ChemSusChem, 2008. 1(5): p. 437-443.

14. Boffa, V., et al., Microporous niobia-silica membranes: Influence of sol composition and structure on gas transport properties. Micropor. Mesopor. Mat., 2009. 118(1-3): p. 202-209.

15. Yoshida, K., et al., Hydrothermal stability and performance of silicazirconia membranes for hydrogen separation in hydrothermal conditions. J. Chem. Eng. Jpn., 2001. 34(4): p. 523-530.

16. Ziolek, M. and I. Nowak, Synthesis and characterization of niobiumcontaining MCM-41. Zeolites, 1997. 18(5-6): p. 356-360.

17. Nowak, I., Frontiers in mesoporous molecular sieves containing niobium: From model materials to catalysts. Catal. Today, 2012. 192(1): p. 80-88.

18. Sedlar, M. and M. Sayer, Reactivity of titanium isopropoxide, zirconium propoxide and niobium ethoxide in the system of 2- 
methoxyethanol, 2,4-pentanedione and water. Journal of Sol-Gel Science and Technology, 1995. 5(1): p. 27-40.

19. Spijksma, G.I., et al., Stabilization and destabilization of zirconium propoxide precursors by acetylacetone. Chem. Commun., 2004. 10(16): p. 1874-1875.

20. Bradley, D.C. and C.E. Holloway, Nuclear magnetic resonance studies on niobium and tantalum penta-alkoxides. J. Chem. Soc. A 1968: p. 219-223.

21. Ibers, J.A., Crystal and Molecular Structure of Titanium (IV) Ethoxide. Nature, 1963. 197(4868): p. 686-687.

22. Blanchard, J., et al., Hydrolysis and Condensation Reactions of Transition Metal Alkoxides: Calorimetric Study and Evaluation of the Extent of Reaction. European J. Inorg. Chem., 1998. 1998(8): p. 1115-1127.

23. Leaustic, A., F. Babonneau, and J. Livage, Structural investigation of the hydrolysis-condensation process of titanium alkoxides Ti(OR)4 $(O R=O P r-i s o, O E t)$ modified by acetylacetone. 1. Study of the alkoxide modification. Chem. Mater., 1989. 1(2): p. 240-247.

24. Pickup, D.M., et al., $A$ structural study of (TiO2) $x$ (SiO2) $1-x(x=$ $0.18,0.30$ and 0.41$)$ xerogels prepared using acetylacetone. J. Mater. Chem., 1999. 9(6): p. 1299-1305.

25. Gervais, C., F. Babonneau, and M.E. Smith, Detection, Quantification, and Magnetic Field Dependence of Solid-State 170 NMR of $X-O-Y(X, Y=S i, T i)$ Linkages: Implications for Characterizing Amorphous Titania-Silica-Based Materials. The Journal of Physical Chemistry B, 2001. 105(10): p. 1971-1977.

26. Delattre, L. and F. Babonneau, 170 Solution NMR Characterization of the Preparation of Sol-Gel Derived SiO2/TiO2 and SiO2/ZrO2 Glasses. Chemistry of Materials, 1997. 9(11): p. 2385-2394.

27. Nowak, I. and M. Ziolek, Niobium Compounds: Preparation, Characterization, and Application in Heterogeneous Catalysis. Chemical Reviews, 1999. 99(12): p. 3603-3624.

28. Bonhomme, C., et al., Advanced Solid State NMR Techniques for the Characterization of Sol-Gel-Derived Materials. Acc. Chem Res., 2007. 40(9): p. 738-746.

29. Drake, K.O., et al., A multinuclear solid state NMR study of the solgel formation of amorphous Nb2O5-SiO2 materials. Solid State Nuc. Mag., 2005. 27(1-2): p. 28-36.

30. Julián, B., et al., Solid-State 170 NMR Characterization of $P D M S-M x O y \quad(M=G e(I V), T i(I V), \quad Z r(I V), \quad N b(V)$, and $T a(V))$ 
Organic-Inorganic Nanocomposites. Chem. Mater., 2004. 16(3): p. 521-529.

31. Holland, M.A., et al., Synthesis, characterisation and performanceof (TiO2) 0.18 (SiO2) 0.82 xerogel catalysts. J. Mater. Chem., 2000. 10(11): p. 2495-2501.

32. Babonneau, F. and J. Maquet, Nuclear magnetic resonance techniques for the structural characterization of siloxane-oxide hybrid materials. Polyhedron, 2000. 19(3): p. 315-322.

33. Cedeño, L., et al., Synthesis of Nb-containing mesoporous silica molecular sieves: Analysis of its potential use in HDS catalysts. Appl. Catal. A: General, 2003. 241(1-2): p. 39-50.

34. Julián, B., et al., Synthesis and Characterization of Transparent PDMS-Metal-Oxo Based Organic-Inorganic Nanocomposites. Chem Mater., 2003. 15(15): p. 3026-3034.

35. Nakamoto, K., Infra red and Raman spectra of Inorganic and coodination compounds. 4 ed, ed. K. Nakamoto1986, New York: John Whiley \& Sons. 259-263.

36. Jiang, H., Z. Zheng, and X. Wang, Kinetic study of methyltriethoxysilane (MTES) hydrolysis by FTIR spectroscopy under different temperatures and solvents. Vib. Spectrosc., 2008. 46(1): p. 1-7.

37. Cai, Y., et al., Electrochemical synthesis, characterization and thermal properties of niobium ethoxide. J. Cent. South Univ. T., 2011. 18(1): p. 73-77.

38. Castricum, H.L., et al., Hydrothermally stable molecular separation membranes from organically linked silica. J. Mater. Chem., 2008. 18(18): p. 2150-2158. 


\section{Crystallized $\mathrm{Nb}_{8} \mathrm{O}_{10}(\mathrm{OEt})_{20}$ from condensation of niobium ethoxide and acetone that exhibits remarkable anisotropy in thermal expansion}




\subsection{Abstract}

Acetone is a useful oxolation source for niobium penta-ethoxide (NPE). Its mild reactivity allows the controlled condensation and crystallization of nanosized $\mathrm{Nb}_{8} \mathrm{O}_{10}(\mathrm{OEt})_{10}$ at room temperature. The condensation process occurs via two complementary routes: (1) aldol condensation and (2) ether elimination. The structure refinement on the crystallites at $T=150 \mathrm{~K}$ and $T=296 \mathrm{~K}$ revealed a remarkable anisotropy in the thermal expansion of $\mathrm{Nb}_{8} \mathrm{O}_{10}(\mathrm{OEt})_{20}$ with a negative expansion coefficient along the $c$-axis. At reduced temperatures the $\mathrm{Nb}_{8} \mathrm{O}_{10}(\mathrm{OEt})_{20}$ clusters were tilted towards the $\underline{a}$ and $c$-axis, most likely driven by a reduction of thermal motion and steric hindrance. Moreover, the irregular shape of the clusters allowed a relatively large compression along the $a$-axis and a relatively large expansion along the $c$-axis.

\subsection{Introduction}

Nanosized molecular clusters are extremely useful building blocks for the bottom-up synthesis of advanced materials in a variety of different applications, such as catalysts, optics, electronics, and membranes [1-6]. By coordination of such clusters with organic linkages or coordination polymers, complex 3D hierarchal and polymeric structures can be synthesized that exhibit well-defined pore sizes and tailor-made functionalities [1-6]. Well defined clusters can be formed through controlled hydrolysis and condensation of metal alkoxides. Yet, conventional hydrolysis and condensation routes have extremely high reactivity, which complicates the synthesis of monodisperse metal-oxo clusters $[7,8]$. Alternatively, water may be replaced by less nucleophilic oxolation sources, such as alcohols, esters and ketones [9-12], which therefore reduced its activation energy: a pathway that is referred as the non-hydrolytic sol-gel (NHSG) route. Even without the use of surfactants well-defined monodisperse crystalline nanosized metal oxides can be synthesized $[13,14]$.

The research presented here was particular focused on nanosized niobium oxo-alkoxo clusters. Nanosized niobia crystallites are well known Brönsted/Lewis acidic catalysts [15-18] and niobia is an $n$-type semiconductor with a band gap of $3.4 \mathrm{eV}$, suitable for applications including 
gas sensing, photocatalysis and dyes for solar cells [18-21]. Despite its potential and contrary to group IV transition metal oxo alkoxide clusters [1, 22], relatively little is known about group $V$ oxo alkoxide clusters and only a few different isolated niobium oxo alkoxo clusters have been reported [2330]. The results presented here demonstrate the formation of high purity $\mathrm{Nb}_{8} \mathrm{O}_{10}(\mathrm{OEt})_{20}$ via a straightforward synthesis and crystallization procedure at room temperature.

The crystal structure was refined at two different temperatures, i.e. $150 \mathrm{~K}$ and $296 \mathrm{~K}$, and was found to exhibit anisotropic thermal expansion behavior, with a negative thermal expansion coefficient along the $c$-axis. Strongly negative thermal expansion behavior is typically observed in frameworks that exhibit strongly anharmonic interatomic or intermolecular potentials along certain orientations, such as metal cyanides [31, 32], metal tungstates/vanadates [33], zeolites [34] and metal organic frameworks [35]. In organic molecules where steep anharmonic potentials are absent and molecules are held together through rather weak Van der Waals (VdW) forces, anisotropic thermal expansion is rarely observed [36, 37]. A remarkable exception is the anisotropic thermal expansion behavior of several acene crystals which are held together solely through VdW forces [36]. These elongated acene molecules were packed together, not exactly plane parallel, but slightly tilted. As illustrated in Figure 6.1 the tilt angle of the elongated molecules increased with increasing temperature due to increased thermal motion and steric hindrance. Consequently, the unit cell elongated particularly along the $a$-axis and to a much smaller extent along the $b$-axis. Among several acene molecules the thermal deformation showed the highest anisotropic behavior for the most elongated molecule that was being analyzed, i.e. pentacene, which was found to have a negative thermal expansion coefficient along the $b$-axis of $\alpha_{b}=-$ $35 \pm 6 \cdot 10^{-6} \quad K^{-1}$. In analogy with pentacene, elongated $\mathrm{Nb}_{8} \mathrm{O}_{10}(\mathrm{OEt})_{20}$ clusters consist of a rather rigid core, where they are packed in a similar

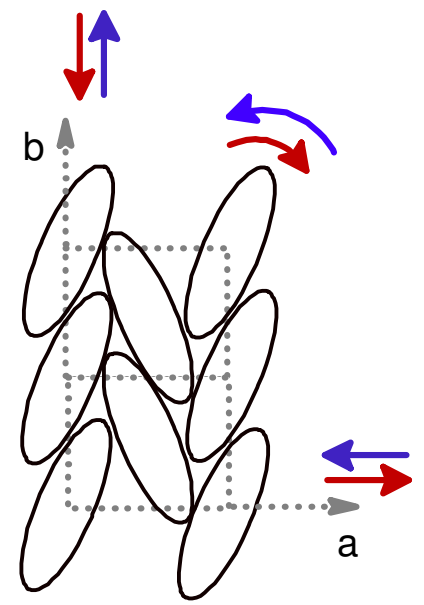

Figure 6.1. Thermal expansion behavior of elongated pentacene molecules as described by Haas et al. [36]. 
tilted arrangement. Due to the surrounding ethoxide groups intermolecular attractive forces are solely rather weak VdW forces. As will be shown below, the thermal expansion behavior is similar to that of acene.

\subsection{Experimental Section}

Before use, toluene (99\%, Alfa Aesar) and acetone $(99,9 \%)$ were dried over anhydrous sodium sulphate $(99,0 \%$, Sigma-Alrich). All samples were prepared inside a glove box under dry nitrogen atmosphere and the crystallites were grown inside $5 \mathrm{~mL}$ polypropylene test tubes with polypropylene screw caps (VWR).

Procedure A: $50 \mathrm{mmol}$ acetone was added to $5 \mathrm{mmol}$ niobium(V) ethoxide (ABCR Chemicals, 99,99\%, abbreviated as NPE). After vigorous shaking the mixture was aged for 1 week at room temperature. Crystallites were removed with tweezers and characterized by ${ }^{1} \mathrm{H}$ and ${ }^{13} \mathrm{C}$ NMR spectroscopy. Procedure B: $5 \mathrm{mmol}$ NPE was dissolved in $30 \mathrm{mmol}$ toluene. While gently stirring the solution with a lab dancer, $20 \mathrm{mmol}$ acetone was added dropwise. After aging the solution for 2 weeks another $10 \mathrm{mmol}$ acetone was added dropwise and then after $2 \mathrm{~h}$ the crystallites were removed with tweezers and characterized by ${ }^{1} \mathrm{H}$ and ${ }^{13} \mathrm{C}$ NMR spectroscopy and by single $\mathrm{X}$-ray diffraction.

At room temperature ( $T=298(3) \mathrm{K}$ ), the data collection was carried out at room temperature using Bruker SMART Apex-II diffractometer with Mo$K_{\alpha}$ radiation $(\lambda=0.71073 \AA$ ). 9356 symmetrically independent reflections were collected in the interval $2.73<2 \theta<29.46$. The structure was solved by direct methods. All metal atom coordinates were located in the initial solution and the oxygen and carbon atoms were found in the subsequent difference Fourier syntheses. All non-hydrogen atoms were refined first isotropically and then anisotropically. Hydrogen atom coordinates were calculated geometrically and the results of their isotropic refinement were included into the final solution. Final discrepancy factors were R1 $=0.0430$ and $w R 2=0.1097$ for the 6419 observed with I > 2 sigma(I). For the measurements at $T=150 \mathrm{~K}$, the crystal was cooled with an Oxford cryosystem liquid nitrogen cooling. 


\subsection{Results and Discussion}

\subsubsection{Synthesis and characterisation of crystallites}

Procedure A: When acetone was added to NPE a white haze formed, which slowly disappeared under vigorous shaking. After 2 days of aging, the first crystallites of $\sim 0.5 \mathrm{~mm}$ diameter were observed by the naked eye and after $\sim 1$ week, the crystallites seemed to have stopped growing. The hexagonal/trigonal prism shaped crystallites (Figure 6.2a) have sizes varying between 1 and $6 \mathrm{~mm}$. The crystallites were characterized by NMR spectroscopy and the characteristic resonances are given in Table 6.1. During aging, the solution changed its colour from colourless to orange/yellow in one week time.
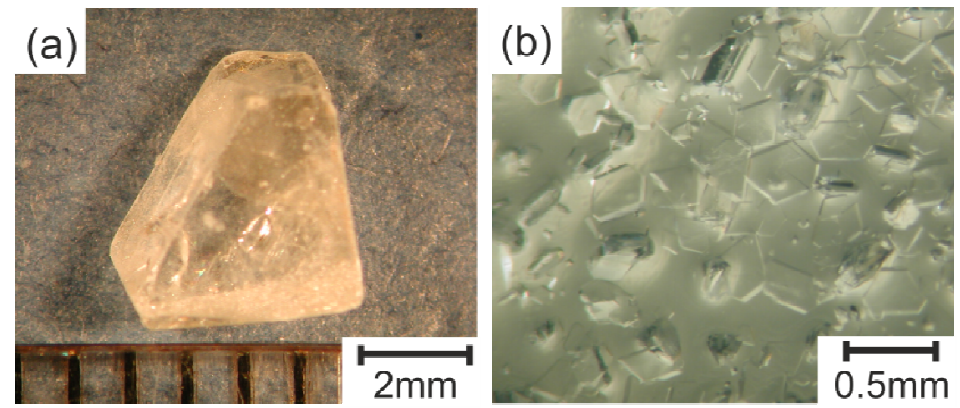

Figure 6.2. Optical images of crystallized $\mathrm{Nb}_{8} \mathrm{O}_{10} \mathrm{OEt}_{20}$ following (a) procedure $\mathrm{A}$, (b) procedure $B$.

Table 6.1. ${ }^{1} \mathrm{H}$ and ${ }^{13} \mathrm{C}$ NMR assignment of $\mathrm{Nb}_{8} \mathrm{O}_{10}(\mathrm{OEt})_{20}$.

\begin{tabular}{llll}
\hline Assignment & & $\delta^{1} \mathrm{H}$ & $\delta^{13} \mathrm{C}$ \\
\hline \multirow{3}{*}{ Bridged ethoxide } & $\mathrm{CH}_{2}$ & $4.67(\mathrm{dq})$ & 70.64 \\
\cline { 2 - 4 } & $\mathrm{CH}_{3}$ & $1.48(\mathrm{~m})$ & \\
\hline \multirow{3}{*}{ Pendant ethoxide } & $\mathrm{CH}_{2}$ & $4.45(\mathrm{~m})$ & 18.45 \\
\cline { 2 - 4 } & $\mathrm{CH}_{3}$ & $1.36(\mathrm{~m})$ & 69.55 \\
\hline
\end{tabular}


${ }^{1} \mathrm{H}-\mathrm{NMR}$ and ${ }^{13} \mathrm{C}-\mathrm{NMR}$ measurements revealed that residual solvent after filtration contained $0.55 \mathrm{~mol}$ 2,2-diethoxypropane and $0.67 \mathrm{~mol}$ mesityl oxide per mol NPE that was added to the solution. Both by-products originated from two different complementary processes: (1) 2,2diethoxypropane, which was most likely being formed through an ether elimination process as reported for $\mathrm{MoO}(\mathrm{OEt})_{4}$ [38]. After coordination of acetone to the $\mathrm{Nb}$-cation (Scheme $6.1 \mathrm{a}_{1}$ ), nucleophilic ethoxy groups were substituted to the enolic carbon atom of acetone (Scheme $6.1 b_{1}, b_{2}$ ). Then, by the release of the enolic oxygen from acetone (EtO) ${ }_{3} \mathrm{Nb}=\mathrm{O}$ was formed, which through further ether elimination yielded $\mathrm{Nb}_{8} \mathrm{O}_{10}(\mathrm{OEt})_{20}$ [24]. (2) Mesityl oxide, a known self-condensation product of acetone, which formed through an aldol addition (Scheme $6.1 \mathrm{c}_{1}$ ), followed by crotonization of diacetone alcohol (Scheme 6.1c $c_{2}$ and $c_{3}$ ) [12, 39]. After two days FTIR experiments revealed a broad $\mathrm{OH}$-stretch vibration at $2480 \mathrm{~cm}^{-1}$ which was associated with $\mathrm{Nb}-\mathrm{OH}$ groups [40] and $\mathrm{Nb}=\mathrm{O}$ vibrations at 920 and $860 \mathrm{~cm}^{-1}$ [24]. Therefore, crotonization may yield both (EtO) ${ }_{3} \mathrm{Nb}=\mathrm{O}$ (Scheme $6.1 \mathrm{c}_{2}$ ) and $(\mathrm{EtO})_{4} \mathrm{Nb}-\mathrm{OH}$ (Scheme 6.1c $\mathrm{c}_{3}$. Subsequently, $\mathrm{Nb}_{8} \mathrm{O}_{10}(\mathrm{OEt})_{20}$ can be formed through further condensation and ether elimination mechanisms.

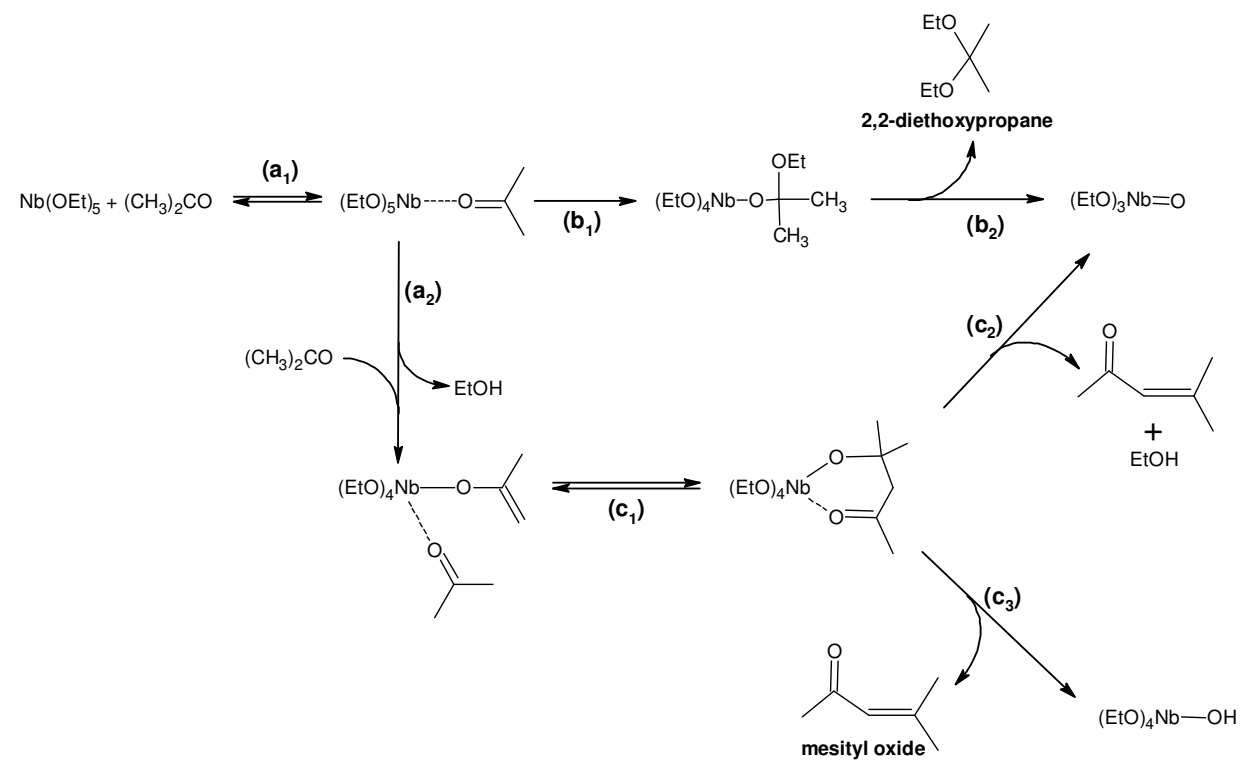

Scheme 6.1. Possible non-hydrolytic hydrolysis pathways of NPE with acetone: $\left(\mathrm{a}_{1}\right)$ Coordination of acetone onto the $\mathrm{Nb}^{5+}$ cation, $\left(\mathrm{a}_{2}\right)$ Ligand exchange between enolic acetone and an ethoxy ligand, $\left(b_{1}\right.$ and $\left.b_{2}\right)$ ether elimination of NPE, $\left(c_{1}\right)$ aldol addition, $\left(c_{2}\right.$ and $c_{3}$ ) crotonization of diacetone alcohol to mesityl oxde. 
Procedure B: During aging the solution for 2 weeks the colour of the solution changed from colourless to orange/yellow. No precipitate/crystallite formed after 0-4 weeks of aging the solution. After 2 weeks of aging crystallization was induced by adding another $10 \mathrm{mmol}$ of acetone. Following, this procedure smaller hexagonal prism shaped crystallites were formed with lateral dimensions in the range between 0.1 $0.5 \mathrm{~mm}$ (Figure 6.2b). These crystallites were found to be more suitable for single $\mathrm{X}$-ray diffraction and gave identical resonance peaks than the crystals from procedure $A$.

\subsubsection{Structure refinement}

By means of single $X$-ray diffraction the crystallites that had formed in procedure $\mathrm{B}$ were identified as $\mathrm{Nb}_{8} \mathrm{O}_{10}(\mathrm{OEt})_{20}$ clusters arranged in a monoclinic unit cell with a $\mathrm{P} 2_{1} / \mathrm{n}$ symmetry. The structure was resolved at two different temperatures, i.e. 150 and $296 \mathrm{~K}$, on two different crystallites. The lattice dimensions are listed in Table 6.2. Similar structures have been reported by Kessler [24] and Bradley [23], however these had been synthesized following other procedures. Kessler synthesized $\mathrm{Nb}_{8} \mathrm{O}_{10}(\mathrm{OEt})_{20}$ by ligand exchange of $\mathrm{NbOCl}_{3}$ with $\mathrm{NaOEt}$, which was followed by ether elimination of $\mathrm{NbO}(\mathrm{OEt})_{3}$ Already in 1968 Bradley and coworkers isolated $\mathrm{Nb}_{8} \mathrm{O}_{10}(\mathrm{OEt})_{20}$ after partial hydrolysis of $\mathrm{Nb}(\mathrm{OEt})_{5}$ [23]. Yet, due to some uncertainties, the structure was not completely resolved. The lattice dimensions were reported but not the exact atomic positions. For the sake of comparison the reported unit cell dimensions are also listed in Table 6.2. The bond angles and bond distances of the resolved structures at $150 \mathrm{~K}$, at $296 \mathrm{~K}$ and the structure derived from $\mathrm{NbOCl}_{3}$ [24] are given for all core atoms in Table $\mathrm{S} 6.1$ and $\mathrm{S6.2}$ of the supplementary information, respectively. We used the same labels as previously reported [24]. However, distorted atoms were represented by $\mathrm{A}$ and B-labels Figure 6.3 for the $150 \mathrm{~K}$ structure and the $\mathrm{C} 7$ label was not being used.

The differences in lattice dimensions between the $296 \mathrm{~K}$ sample and the $\mathrm{NbOCl}_{3}$-derived sample [24] were substantially smaller as compared to structural differences at 150 and $296 \mathrm{~K}$. Among the structures being resolved at different temperatures a remarkable anisotropic deformation was observed. With a decreasing temperature both the $a$ and $b$ axis reduced in length, i.e. $\alpha_{a} \sim 2.3 \cdot 10^{-6} \mathrm{~K}^{-1}$ and $\alpha_{b} \sim 104 \cdot 10^{-6} \mathrm{~K}^{-1}$ ), while the $c$-axis 
increased in length $\left(\alpha_{c} \sim-47 \cdot 10^{-6} \mathrm{~K}^{-1}\right)$. On the other hand, bond angles and bond distances between niobium and the bridging oxygen atoms (01-08) changed with less than 1\% (Table 6.3), Appendix Table A1 and A2). Therefore, the deformation was probably not induced by distortion of the $\mathrm{Nb}$ octahedra. Instead, the anisotropy in thermal expansion in this case is probably caused by differences in VdW forces between clusters along different directions. The directions along which the intermolecular interactions between clusters are relatively weak allow a larger expansions as compared to the directions along which these interactions are relatively strong [41].

Table 6.2. Comparison of lattice parameters between several refined structures of $\mathrm{Nb}_{8} \mathrm{O}_{10} \mathrm{OEt}_{20}$.

\begin{tabular}{|c|c|c|c|c|c|}
\hline \multicolumn{2}{|c|}{ Synthesized from } & $\begin{aligned} & \mathrm{Nb}(\mathrm{OEt})_{5} \\
+ & \left(\mathrm{CH}_{3}\right)_{2} \mathrm{CO}\end{aligned}$ & $\begin{aligned} & \mathrm{Nb}(\mathrm{OEt})_{5} \\
+ & \left(\mathrm{CH}_{3}\right)_{2} \mathrm{CO}\end{aligned}$ & $\begin{array}{l}\mathrm{NbOCl}_{3} \\
+\mathrm{NaOEt}\end{array}$ & $\begin{array}{l}\text { Hydrolysis of } \\
\mathrm{Nb}(\mathrm{OEt})_{5}\end{array}$ \\
\hline \multicolumn{2}{|c|}{$\begin{array}{l}\text { Temperature } \\
\text { diffraction } \\
\text { measurements }\end{array}$} & $150 \mathrm{~K}$ & $296 \mathrm{~K}$ & $283-303 \mathrm{~K}$ & $283-303 \mathrm{~K}$ \\
\hline \multicolumn{2}{|c|}{ Reference } & This work & This work & [24] & [23] \\
\hline \multicolumn{2}{|c|}{$\begin{array}{l}\text { Symmetry space } \\
\text { group H-M }\end{array}$} & P 21/n & P 21/n & P 21/n & P 21/n \\
\hline \multicolumn{6}{|c|}{ Lattice parameters } \\
\hline \multirow{3}{*}{$\begin{array}{l}\text { cell lengths } \\
\text { (A) }\end{array}$} & $a$ & $14.9279(12)$ & $14.933(3)$ & 14.9169(9) & 14.96 \\
\hline & $b$ & $14.0269(15)$ & $14.242(3)$ & $14.2541(7)$ & 14.36 \\
\hline & c & $16.9730(15)$ & $16.856(3)$ & $16.8726(8)$ & 16.84 \\
\hline \multirow{2}{*}{$\begin{array}{l}\text { cell angles } \\
\left({ }^{\circ}\right)\end{array}$} & $\alpha, \gamma$ & 90 & 90 & 90 & 90 \\
\hline & $\beta$ & $91.733(4)$ & $91.577(2)$ & $91.754(4)$ & 91.0 \\
\hline \multicolumn{2}{|l|}{$\begin{array}{l}\text { cell volume } \\
\left(\AA^{3}\right)\end{array}$} & $3402.3(6)$ & $3583.7(12)$ & $3585.9(3)$ & 3617 \\
\hline
\end{tabular}

Since the niobium-oxo core is well shielded by ethoxide groups clustercluster interactions are dominated by VdW forces which strongly decay with increasing atomic distances. The intermolecular carbon-carbon distances in reveal several short range interactions in the range between 3.7 and $4.5 \AA$ along the [100], [010], [111] and [111] directions (Table 6.4). In contrast, the shortest and second shortest C-C distances at $296 \mathrm{~K}$ along the [001] direction were 5.4 and $7.2 \AA$, respectively. As is also illustrated in Figure 6.4a and Figure 6.4b, the gap along the [001] is substantially larger than along [100] and [010]. At lower temperatures, Van der Waals 
interactions become increasingly important, causing contraction along the [100] and [010] axes due to stronger VdW interactions than in the [001] direction. Since clusters are packed in a body centered cube-like geometry, compression along [100] and [010] cause stress along close-packed [111] and [111] diagonals (Figure 6.4a). Consequently, the stress along the diagonals causes expansion of clusters along [001].

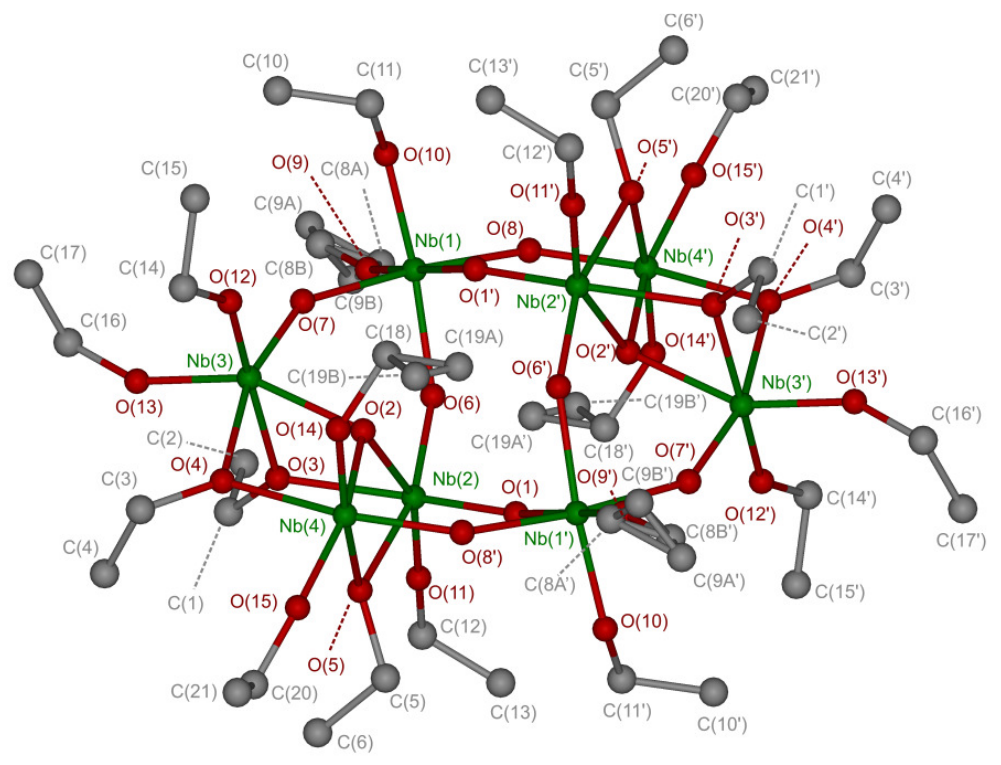

Figure 6.3. Assignment of the resolved $\mathrm{Nb}_{8} \mathrm{O}_{10}(\mathrm{OEt})_{20}$ structure measured at $150 \mathrm{~K}$.

The compression along the $\langle 111\rangle$ diagonals also caused a screw-like rotation of the clusters. In analogy with acene crystals, the elongated direction of the molecules become more aligned upon reducing the temperature. The direction of elongation of the rigid core could be described by a vector going from the origin of inversion symmetry to the $\mathrm{O} 4$ atom (Figure 6.3). Note that pendant oxygen atoms that are more flexible were not considered to be part of the rigid core. 
Table 6.3. Selection of relevant bond angles of the $\mathrm{Nb}_{8} \mathrm{O}_{10}(\mathrm{OEt})_{20}$ structures.

\begin{tabular}{|c|c|c|c|c|c|}
\hline \multirow[b]{2}{*}{ Synthesized from } & \multicolumn{3}{|c|}{ Bond angles } & \multicolumn{2}{|c|}{ Differences $\left({ }^{\circ}\right)$} \\
\hline & $\mathrm{Nb}(\mathrm{OEt})_{5}$ & $\mathrm{Nb}(\mathrm{OEt})_{5}$ & $\mathrm{NbOCl}_{3}$ & $\begin{array}{l}150 \mathrm{~K} \\
\text { vs. }\end{array}$ & $\begin{array}{c}\mathrm{NbOCl}_{3} \\
\text { vs. }\end{array}$ \\
\hline Reference & This work & This work & [24] & & \\
\hline Nb1-O1-Nb2 & $144.61(9)$ & $143.75(16)$ & $144.2(2)$ & 0.9 & 0.4 \\
\hline $\mathrm{Nb1-06-Nb2}$ & $147.02(10)$ & $147.28(16)$ & $147.1(2)$ & -0.3 & -0.2 \\
\hline Nb1-07-Nb3 & $145.74(10)$ & $146.08(18)$ & $146.3(2)$ & -0.3 & 0.2 \\
\hline $\mathrm{Nb1-08-Nb4}$ & $144.86(9)$ & $144.70(17)$ & $144.3(3)$ & 0.2 & -0.4 \\
\hline $\mathrm{Nb2-O5-Nb4}$ & $101.54(7)$ & $101.32(12)$ & 101.42(19) & 0.2 & 0.1 \\
\hline $\mathrm{Nb2}-\mathrm{O} 2-\mathrm{Nb} 3$ & $106.95(7)$ & 107.14(13) & $106.9(2)$ & -0.2 & -0.2 \\
\hline $\mathrm{Nb2-O2-Nb4}$ & $108.24(7)$ & 108.19(13) & 107.79(17) & 0.0 & -0.4 \\
\hline $\mathrm{Nb2-O3-Nb3}$ & $102.23(7)$ & $102.12(12)$ & $102.25(18)$ & 0.1 & 0.1 \\
\hline Nb3-O2-Nb4 & $111.49(7)$ & 111.39(13) & 111.62(19) & 0.1 & 0.2 \\
\hline $\mathrm{Nb3}-\mathrm{O} 4-\mathrm{Nb} 4$ & $103.40(7)$ & $103.19(12)$ & 103.33(19) & 0.2 & 0.1 \\
\hline O6-Nb1-09 & $95.59(8)$ & $92.77(14)$ & $93.2(2)$ & 2.8 & 0.4 \\
\hline O6-Nb1-010 & $164.89(8)$ & $166.00(16)$ & 166.49(19) & -1.1 & 0.5 \\
\hline 07-Nb1-09 & $94.92(8)$ & $93.80(15)$ & $94.54(19)$ & 1.1 & 0.7 \\
\hline O7-Nb1-010 & $86.37(8)$ & $85.59(16)$ & $85.90(17)$ & 0.8 & 0.3 \\
\hline O1-Nb1-O7 & $89.68(7)$ & $88.36(12)$ & $88.47(17)$ & 1.3 & 0.1 \\
\hline 08-Nb1-09 & $90.49(8)$ & $92.44(15)$ & 91.61(19) & -2.0 & -0.8 \\
\hline O9-Nb1-010 & $95.31(9)$ & $95.17(17)$ & $94.6(2)$ & 0.1 & -0.6 \\
\hline 01-Nb1-09 & $175.34(8)$ & $176.14(13)$ & 175.73(19) & -0.8 & -0.4 \\
\hline O1-Nb1-010 & $85.72(8)$ & $88.18(16)$ & $88.61(17)$ & -2.5 & 0.4 \\
\hline $\mathrm{O} 2-\mathrm{Nb} 4-\mathrm{O} 14$ & $94.07(7)$ & $96.13(13)$ & $95.57(18)$ & -2.1 & -0.6 \\
\hline O5-Nb4-014 & $168.23(7)$ & $170.69(14)$ & $170.56(19)$ & -2.5 & -0.1 \\
\hline O14-Nb4-O15 & $100.38(8)$ & $98.77(18)$ & $98.8(2)$ & 1.6 & 0.0 \\
\hline O8-Nb4-014 & $93.44(8)$ & $92.30(15)$ & 92.95(19) & 1.1 & 0.7 \\
\hline
\end{tabular}

Consequently, the screw-like rotation with decreasing temperature from 296 to $150 \mathrm{~K}$ can be described by two rotations: Firstly, a clockwise rotation of $\theta_{04}=1.605^{\circ}$ along the unit vector $\mathrm{u}_{{ }^{\circ} 04}=[0.769,-0.025,0.639]$. The unit vector was chosen perpendicular to both $\mathrm{O} 4$ vectors to align the $\mathrm{O} 4$ at 296 $\mathrm{K}$ to the $\mathrm{O} 4$ at $150 \mathrm{~K}$. Secondly, a counter-clockwise rotation of $\phi_{04}=0.662^{\circ}$ along the unit vector $u_{04}=[0.395,0.804,-0.445]$ that was chosen in line with the $\mathrm{O} 4$ vector to align $\mathrm{Nb1}$ atoms. For the sake of comparison an overlay of packed clusters was made within the (110) plane at 150 and 296 $\mathrm{K}$, with the right bottom cluster being aligned using the above described rotation angles, see Figure 6.5. As compared to the $296 \mathrm{~K}$ structure the 150 $\mathrm{K}$ structure is tilted, causing clusters to be packed more closely along the 
[110] direction. Due to reduction of the tilt angle with respect to the [001] direction the crystal structure was elongated along the [001] direction.

Table 6.4. Intermolecular distances $(\AA)$ between specific carbon atoms between $\mathrm{Nb}_{8} \mathrm{O}_{10}(\mathrm{OEt})_{20}$ clusters.

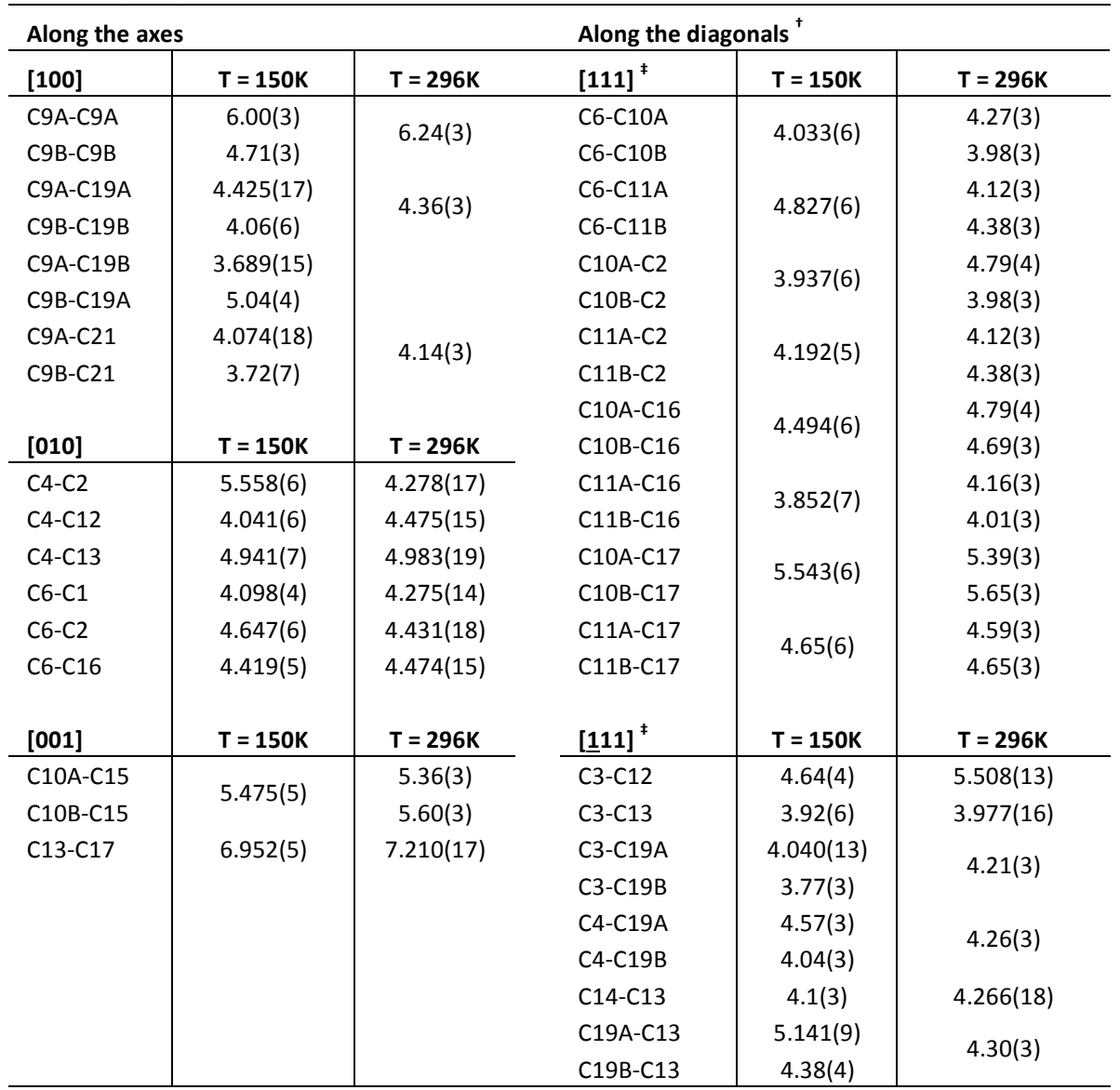

† Distance from $(x, y, z)$ to $(1 / 2-x, 1 / 2+y, 1 / 2-z)$ positions.

${ }^{\ddagger}$ Due to symmetry $[111]=[1 \underline{1} 1]$ and $[\underline{111}]=[\underline{11} 1]$ 

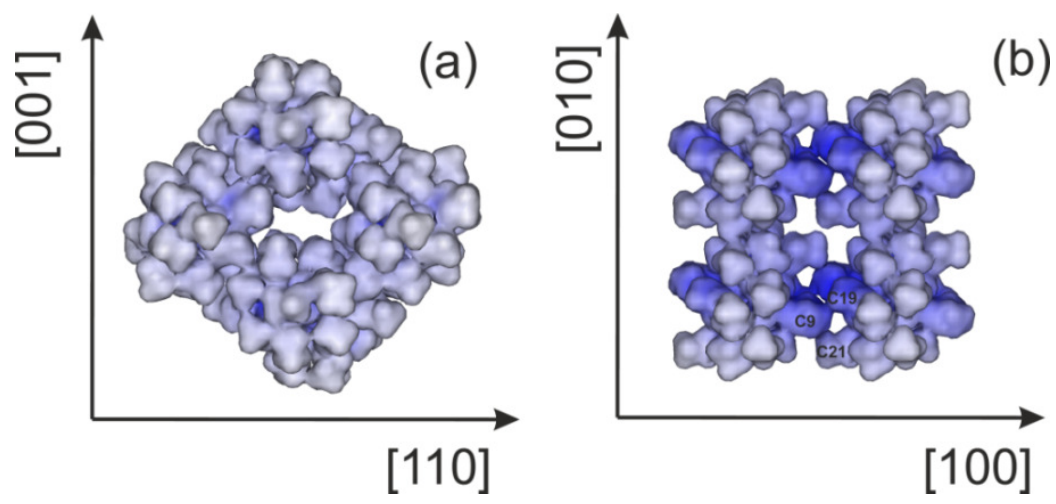

Figure 6.4 Surface exposed representation of packed $\mathrm{Nb}_{8} \mathrm{O}_{10}(\mathrm{OEt})_{20}$ clusters at 150 $\mathrm{K}$ : (a) 4 clusters packed within the (110) plane and (b) 4 clusters packed within the (001) plane.

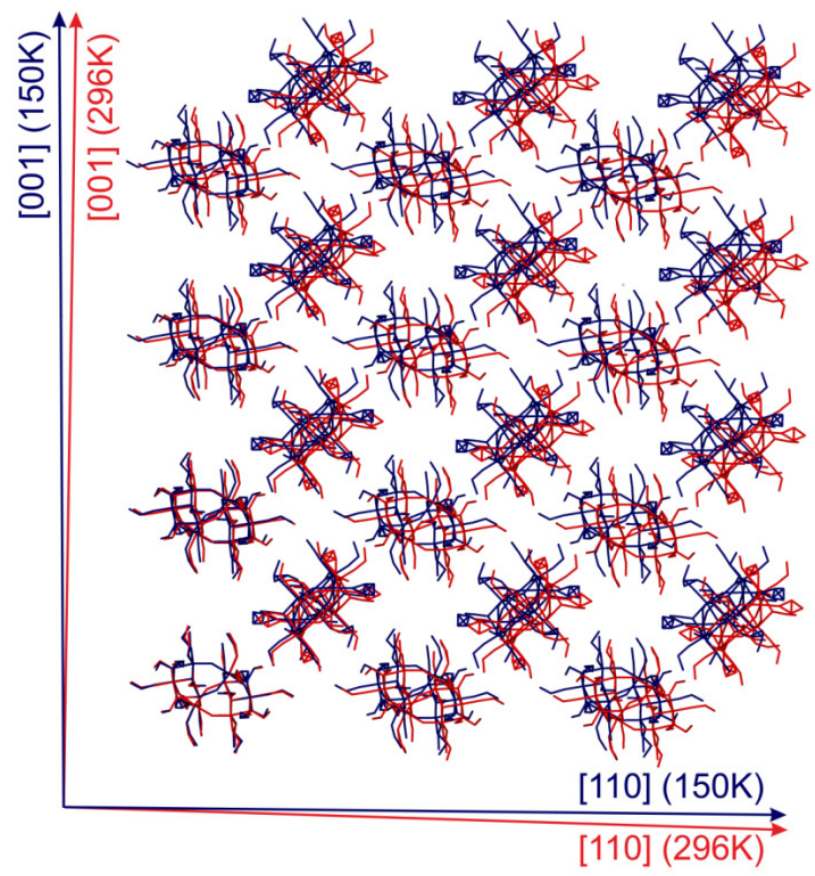

Figure 6.5. Comparison of stacking of $\mathrm{Nb}_{8} \mathrm{O}_{10}(\mathrm{OEt})_{20}$ clusters at $150 \mathrm{~K}$ and $296 \mathrm{~K}$, respectively, within the (110) plane, with the right bottom clusters being aligned with each other by clockwise rotations: $\theta_{04}=1.605^{\circ}$ along the unit vector $\mathrm{u}_{\perp_{04}}=$ $[0.769,-0.025,0.639], \phi_{04}=0.662^{\circ}$ along the unit vector $u_{\| 04}=[0.395,0.804,-$ $0.445]$, in line with the 04 vector. 
Moreover, the contraction along the $b$-axis is substantially larger than along the $a$-axis. In comparison with the previous reported structure [24] the $a$ axis was even slightly larger while the temperature was lower. To understand the cause of this large difference we may consider the niobiumoxo core as an ellipsoidal cage with $\mathrm{O} 4, \mathrm{Nb} 1$ and $\mathrm{Nb2}$ that span this cage, as illustrated in Figure 6.6. The vector $\mathbf{v}_{1}=[1.82,3.92,-2.04]$ was described as the vector going from the origin of inversion to $O(4), v_{2}=[-2.36,0.20,-1.72]$ was described as an orthogonal vector to $\mathbf{v}_{1}$ such that the ellipse that spans $\left\{\mathbf{v}_{1}, \mathbf{v}_{2}\right\}$ crosses Nb1, and $\mathbf{v}_{3}=[-0.84,1.05,1.24]$ was described such that Nb2 lies within the ellipsoidal surface that spans $\left\{\mathbf{v}_{1}, \mathbf{v}_{2}, \mathbf{v}_{3}\right\}$. Moreover, $\mathbf{v}_{4}$ is the vector within the ellipse that spans $\left\{\mathbf{v}_{2}, \mathbf{v}_{3}\right\}$ and is perpendicular to the

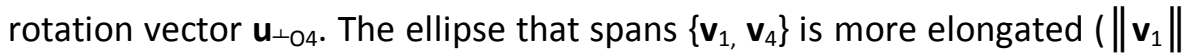
/ $\left\|\mathbf{v}_{4}\right\|=2.57$ ) than the ellipse that spans $\left\{\mathbf{v}_{2}, \mathbf{v}_{3}\right\}$ with $\left\|\mathbf{v}_{2}\right\| /\left\|\mathbf{v}_{3}\right\|=1.57$ ). Therefore, the $\theta_{04}$ rotation along $\mathrm{u}_{{ }^{O} \mathrm{O}}$ has a more profound effect on the thermal expansion as compared to the $\phi_{04}$ rotation. Upon cooling the $\theta_{04}$ rotation caused the $v_{1}$ vector to engage both the $a$ and $c$ axis with 0.069 and $0.086 \AA$, respectively, and it moved $0.076 \AA$ away from the $b$-axis. The $\theta_{04}$ rotation explained the substantial shrinkage along $b$, however it would cause a similar degree of expansion along both $a$ and $c$ upon cooling. In addition, the $\phi_{04}$ rotation caused the vector $\mathbf{v}_{2}$ to approach the $a$-axis which would also imply a small increase of the $a$-axis upon cooling.

The limited expansion along the $a$-axis can be explained by the irregular shape of the $\mathrm{Nb}_{8} \mathrm{O}_{10}(\mathrm{OEt})_{20}$ cluster. Upon contraction along the $a$-axis the $\mathrm{C} 9$ atom of an ethoxide group being attached to $\mathrm{Nb} 1$ penetrated in between $\mathrm{C} 21$ and $\mathrm{C} 19$ of ethoxide groups being attached to $\mathrm{Nb3}$ of its adjacent cluster along the $a$-axis as illustrated in Figure 6.4b. Hence, it allows Nb1 to approach its neighbouring Nb3 from $10.19 \AA$ at $296 \mathrm{~K}$ to $9.47 \AA$ at $150 \mathrm{~K}$ without paying a very high penalty in terms of repulsive forces between the ethoxide groups.

However, this compression caused some disorder in the case of both C9 and C19. Due to the reduced amount of available space the ethoxide groups of these carbon atoms may have difficulties finding their optimal geographical configuration, which allows for the existence of two 
configurations. Moreover, $\mathrm{O}-\mathrm{Nb}-\mathrm{O}$ bond angles revealed a substantial deformation of the ethoxide groups being attached to $\mathrm{Nb1}$, i.e. the 09-C8$\mathrm{C} 9$ and the 010-C11-C10 ethoxide groups (Table 6.3) 09 engaged 08 with $2.0^{\circ}$ and $\mathrm{O} 10$ engaged $\mathrm{O} 1$ with $2.4^{\circ}$, while the angle $09-\mathrm{Nb1}-\mathrm{O} 10$ remained almost constant. Thus, the interaction between C9 with both C19 and C21 of its adjacent cluster caused a tilt of the 09 atom, which engaged the 010 atom that consequently tilted as well. In addition, the O2-Nb4-O14 decreased with $2.0^{\circ}$ and the $015-\mathrm{Nb} 4-014$ increased with $1.6^{\circ}$. The deformation of the 014-C18-C19 ethoxide was most likely also a consequence of the C19-C9 interference of adjacent clusters along the $a$ axis at a reduced temperature.

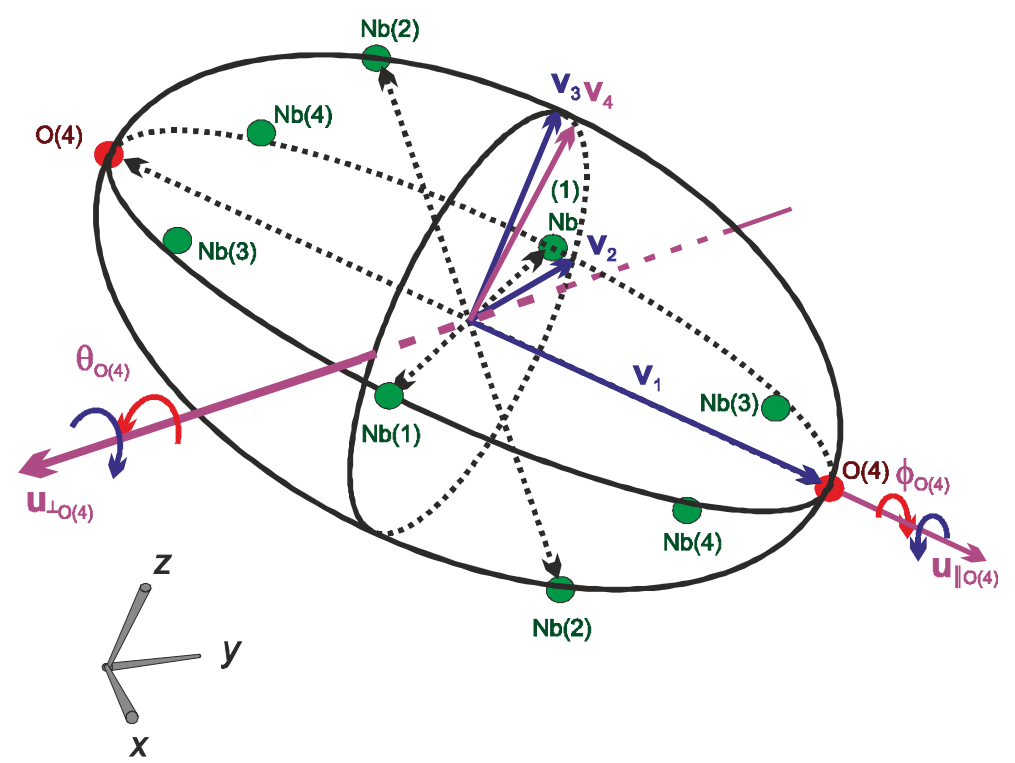

Figure 6.6. Sketch of core atoms that span an ellipsoidal cage which is described by the orthogonal vectors $v_{1}, v_{2}$ and $v_{3}$.

Nevertheless, the results should be interpreted with caution, since the measurements were done on different crystallites. The clusters were held together solely through relatively weak VdW forces, while strong directional forces such as hydrogen bonds were absent. Therefore, we cannot exclude the coexistence of metastable phases with clusters that were packed under a slightly different angle. Moreover, subtle differences 
were observed with a previously reported structure of $\mathrm{Nb}_{8} \mathrm{O}_{10}(\mathrm{OEt})_{20}$ [24] and the structure refined at $296 \mathrm{~K}$. Especially, of ethoxide groups along the $a$-axis, where differences of $0.8^{\circ}$ and $0.7^{\circ}$ were observed in the case of the 08-Nb1-09 and 04-Nb4-014 bond angles respectively. To ensure that the deformation is predominantly caused by a difference in temperature the structure should be refined on the same crystal at different temperatures.

\subsection{Conclusions}

$\mathrm{Nb}_{8} \mathrm{O}_{10}(\mathrm{OEt})_{20}$ was synthesized through a slow and controlled condensation of niobium(V)ethoxide with acetone as a oxolation source at room temperature. It confirms the potential of acetone for controlled condensation of metal alkoxides. This condensation occurred to a similar extent via two complementary pathways: (1) ether elimination and (2) aldol condensation.

A comparison between the structures being refined at 150 and $296 \mathrm{~K}$ revealed a remarkable anisotropy in the thermal expansion with negative thermal expansion along the $c$-axis. The anisotropy originates from the following factors: (1) Weak VdW interactions along the $c$-axis due to the relative large atom-atom distances, which allow large expansions; (2) A screw-like tilt of the rigid and elongated niobium oxo core, which was most likely driven by reducing thermal motion and steric hindrance at reducing temperatures; (3) The irregular shape of the clusters that allow a relatively large compression along the $a$-axis.

\subsection{References}

1. Rozes, L. and C. Sanchez, Titanium oxo-clusters: precursors for a Lego-like construction of nanostructured hybrid materials. Chem Soc Rev, 2011. 40(2): p. 1006-1030.

2. Sanchez, C., et al., "Chimie douce": A land of opportunities for the designed construction of functional inorganic and hybrid organicinorganic nanomaterials. C.R. Chim., 2010. 13(1-2): p. 3-39.

3. Sanchez, C., et al., Designed Hybrid Organic-Inorganic Nanocomposites from Functional Nanobuilding Blocks. Chem Mater., 2001. 13(10): p. 3061-3083. 
4. Schubert, U., Cluster-based inorganic-organic hybrid materials. Chem. Soc. Rev., 2011. 40(2): p. 575-582.

5. Schubert, U., Polymers Reinforced by Covalently Bonded Inorganic Clusters. Chem. Mater., 2001. 13(10): p. 3487-3494.

6. Tranchemontagne, D.J., et al., Secondary building units, nets and bonding in the chemistry of metal-organic frameworks. Chem. Soc. Rev., 2009. 38(5): p. 1257-1283.

7. Sanchez, C., et al., Chemical modification of alkoxide precursors. J. Non-Cryst. Solids, 1988. 100(1): p. 65-76.

8. Livage, J., M. Henry, and C. Sanchez, Sol-gel chemistry of transition metal oxides. Prog. Solid State Ch., 1988. 18(4): p. 259-341.

9. Mutin, P.H. and A. Vioux, Nonhydrolytic Processing of Oxide-Based Materials: Simple Routes to Control Homogeneity, Morphology, and Nanostructure. Chem. Mater., 2009. 21(4): p. 582-596.

10. Vioux, A., Nonhydrolytic sol-gel routes to oxides. Chem. Mater., 1997. 9(11): p. 2292-2299.

11. Goel, S.C., et al. New chemistry for the sol-gel process: Acetone as a new condensation reagent. in MRS Proceedings. 1992. Cambridge Univ Press.

12. Steunou, N., et al., Ketones as an oxolation source for the synthesis of titanium-oxo-organoclusters. New J Chem., 1999. 23(11): p. 1079-1086.

13. Niederberger, M. and G. Garnweitner, Organic Reaction Pathways in the Nonaqueous Synthesis of Metal Oxide Nanoparticles. Chem. Eur. J., 2006. 12(28): p. 7282-7302.

14. Pinna, N. and M. Niederberger, Surfactant-Free Nonaqueous Synthesis of Metal Oxide Nanostructures. Angew. Chem. Int. Edit., 2008. 47(29): p. 5292-5304.

15. Wachs, I.E., et al., Molecular structure and reactivity of the Group $V$ metal oxides. Catal. Today, 2003. 78(1-4): p. 13-24.

16. Nowak, l., et al., Catalytic properties of niobium and gallium oxide systems supported on MCM-41 type materials. Appl. Catal. A: General, 2007. 325(2): p. 328-335.

17. Carniti, P., A. Gervasini, and M. Marzo, Dispersed NbOx Catalytic Phases in Silica Matrixes: Influence of Niobium Concentration and Preparative Route. J. Phys. Chem. C, 2008. 112(36): p. 14064-14074.

18. Tsang, E., et al., Nanostructured Nb 205 catalysts. Nano Rev., 2012. 3. 
19. Wang, Y.-D., et al., Effects of calcining temperature on lattice constants and gas-sensing properties of $N b<$ sub $>2</$ sub $>0<$ sub $>$ 5</sub>. Mater. Lett., 2001. 49(5): p. 277-281.

20. Prado, A.G., et al., $N b<$ sub $>2</ s u b>0<s u b>5</$ sub $>$ as efficient and recyclable photocatalyst for indigo carmine degradation. Appl. Catal. B, 2008. 82(3): p. 219-224.

21. Jose, R., V. Thavasi, and S. Ramakrishna, Metal Oxides for DyeSensitized Solar Cells. J Am. Ceram. Soc., 2009. 92(2): p. 289-301.

22. Cotton, S.A., Titanium, zirconium and hafnium. Annu. Rep. A, 2006. 102(0): p. 171-180.

23. Bradley, D., M. Hursthouse, and P. Rodesiler, The structure of a crystalline niobium oxide ethoxide, Nb8010 (OEt) 20. Chem. Commun. 1968(18): p. 1112-1113.

24. Kessler, V.G., et al., The structure of $\mathrm{Nb}_{8} \mathrm{O}_{10}(\mathrm{OEt})_{20}$ and the nature of crystalline metal alkoxides. Russ. J. Inorg. Chem., 1991. 36(7): p. 7.

25. Hubert-Pfalzgraf, L.G., et al., Metal alkoxides with polymerizable ligands: synthesis and molecular structure of $[N b 4(\mu-O) 4(\mu, \eta 2-$ O2CMe-CH2)4(OPri)8]. Polyhedron, 1997. 16(4): p. 581-585.

26. Hubert-Pfalzgraf, L., Hydroxyethylmethacrylate as a source of ethyleneglycolate ligands. Synthesis and characterization of $\mathrm{Nb4}(A)^{\prime}$ $\eta 1, \eta 2-O C 2 H 4 O) 2$ (Á3, $\eta 1, \eta 2-O C 2 H 4 O) 2$ (Á-OC2H4O)(OPri) 10. J. Chem. Soc., Dalton Transactions, 1999(15): p. 2407-2408.

27. Boyle, T.J., et al., Crystallographic characterization of the esterification pathway of Group V alkoxides. Polyhedron, 2002. 21(23): p. 2333-2345.

28. Steunou, N., et al., A tetranuclear niobium oxo acetate complex. Synthesis, $X$-ray crystal structure, and characterization by solid-state and liquid-state NMR spectroscopy. Inorg. Chem., 1998. 37(5): p. 901-910.

29. Boyle, T.J., et al., Niobium (V) Alkoxides. Synthesis, Structure, and Characterization of $[\mathrm{Nb}(\mu-\mathrm{OCH} 2 \mathrm{CH} 3)(\mathrm{OCH} 2 \mathrm{C}(\mathrm{CH} 3)$ 3) 4] $2,\{[\mathrm{H} 3 \mathrm{CC}$ $(\mathrm{CH} 2 \mathrm{O})(\mathrm{CH} 2-\mu-\mathrm{O})(\mathrm{C}(\mathrm{O}) 2)] \mathrm{Nb} 2(\mu-\mathrm{O})(\mathrm{OCH} 2 \mathrm{CH} 3) 5\} 2$, and $\{[\mathrm{H} 3 \mathrm{CC}$ (CH2O) 2 (CH2- $\mu-\mathrm{O})] \mathrm{Nb}(\mathrm{OCH} 2 \mathrm{CH} 3) 2\} 2$ for Production of Mixed Metal Oxide Thin Films1. Chem. Mater., 1997. 9(12): p. 3187-3198.

30. Herbst, L., et al., cyclo-Tetra--oxido-tetrakis [(acetylacetonato-20, $\left.O^{\prime}\right)$ bis (ethanolato-O) niobium (V)]. Acta Crystallographica Section E: Structure Reports Online, 2011. 67(12): p. m1669-m1670.

31. Goodwin, A.L., et al., Colossal positive and negative thermal expansion in the framework material Ag3 [Co (CN) 6]. Science, 2008. 319(5864): p. 794-797. 
32. Phillips, A.E., et al., Nanoporosity and Exceptional Negative Thermal Expansion in Single-Network Cadmium Cyanide. Angew. Chem., 2008. 120(8): p. 1418-1421.

33. S. O. Evans, J., Negative thermal expansion materials [dagger]. J. Chem. Soc., Dalton Transactions, 1999(19): p. 3317-3326.

34. Attfield, M.P. and A.W. Sleight, Exceptional Negative Thermal Expansion in AIPO4-17. Chem. Mater., 1998. 10(7): p. 2013-2019.

35. Dubbeldam, D., et al., Exceptional negative thermal expansion in isoreticular metal-organic frameworks. Angew. Chem., 2007. 119(24): p. 4580-4583.

36. Haas, S., et al., Large uniaxial negative thermal expansion in pentacene due to steric hindrance. Phys. Rev. B, 2007. 76(20): p. 205203.

37. Smeets, S. and M. Lutz, Hexakis(urea-[kappa]O)zinc(II) dinitrate at 110 and $250 \mathrm{~K}$ : uniaxial negative thermal expansion. Acta Crystallogr. C, 2011. 67(2): p. m50-m55.

38. Kessler, V.G., K.V. Nikitin, and A.I. Belokon, $A$ new argument in favor of the ether elimination mechanism: formation of acetals on action of molybdenum alkoxides on carbonyl compounds. Polyhedron, 1998. 17(13): p. 2309-2311.

39. Salvapati, G., K. Ramanamurty, and M. Janardanarao, Selective catalytic self-condensation of acetone. J. Mol. Catal., 1989. 54(1): p. 9-30.

40. Sathyanarayana, D. and C. Patel, Studies on the Hydroxide and Peroxide of Niobium. Z. Anorg. Allg. Chem., 1967. 353(1-2): p. 103108.

41. Salud, J., et al., Anisotropy of intermolecular interactions from the study of the thermal-expansion tensor. J. Appl. Crystallogr., 1998. 31(5): p. 748-757. 


\section{S6.) Supporting information}

Crystallized Nb8010(OEt)20 from condensation of niobium ethoxide and acetone that exhibits remarkable anisotropy in thermal expansion 


\section{S6.1. Extended lists of bond lengths and angles}

Table S6.1. Bond lengths of $\mathrm{Nb}_{8} \mathrm{O}_{10}(\mathrm{OEt})_{20}$ structures.

\begin{tabular}{|c|c|c|c|c|c|}
\hline \multirow{3}{*}{$\begin{array}{l}\text { Synthesized } \\
\text { from }\end{array}$} & \multicolumn{3}{|c|}{ Bond lengths } & \multicolumn{2}{|c|}{ Relative difference } \\
\hline & $\mathrm{Nb}(\mathrm{OEt})_{5}$ & $\mathrm{Nb}(\mathrm{OEt})_{5}$ & $\mathrm{NbOCl}_{3}$ & $\begin{array}{c}150 \mathrm{~K} \\
\text { vs. }\end{array}$ & $\begin{array}{c}\mathrm{NbOCl}_{3} \\
\text { vs. }\end{array}$ \\
\hline & $+\left(\mathrm{CH}_{3}\right)_{2} \mathrm{CO}$ & $+\left(\mathrm{CH}_{3}\right)_{2} \mathrm{CO}$ & $+\mathrm{NaOEt}$ & $296 K$ & $\mathrm{Nb}(\mathrm{OEt})_{5}$ \\
\hline$T$ & $150 \mathrm{~K}$ & $296 \mathrm{~K}$ & $283-303 \mathrm{~K}$ & & \\
\hline Reference & This work & This work & [1] & & \\
\hline $\mathrm{Nb1-O1}$ & $2.0439(16)$ & $2.044(3)$ & $2.039(4)$ & $0.0 \%$ & $-0.2 \%$ \\
\hline $\mathrm{Nb1-O6}$ & $1.9929(17)$ & $2.000(3)$ & $1.994(4)$ & $-0.4 \%$ & $-0.3 \%$ \\
\hline $\mathrm{Nb1-07}$ & $2.0486(17)$ & $2.045(3)$ & $2.054(4)$ & $0.2 \%$ & $0.4 \%$ \\
\hline $\mathrm{Nb1-O8}$ & $1.9742(17)$ & $1.959(3)$ & $1.976(4)$ & $0.8 \%$ & $0.9 \%$ \\
\hline Nb1-O9 & $1.867(2)$ & $1.869(3)$ & $1.839(4)$ & $-0.1 \%$ & $-1.6 \%$ \\
\hline $\mathrm{Nb1-010}$ & $1.8994(18)$ & $1.885(4)$ & $1.889(5)$ & $0.8 \%$ & $0.2 \%$ \\
\hline $\mathrm{Nb2-O1}$ & $1.8286(17)$ & $1.826(3)$ & $1.831(4)$ & $0.1 \%$ & $0.3 \%$ \\
\hline $\mathrm{Nb} 2-\mathrm{O} 2$ & $2.0869(16)$ & $2.083(3)$ & $2.103(4)$ & $0.2 \%$ & $1.0 \%$ \\
\hline $\mathrm{Nb} 2-\mathrm{O} 3$ & $2.1772(17)$ & $2.179(3)$ & $2.191(5)$ & $-0.1 \%$ & $0.6 \%$ \\
\hline $\mathrm{Nb} 2-\mathrm{O} 5$ & $2.1791(17)$ & $2.194(3)$ & $2.199(4)$ & $-0.7 \%$ & $0.2 \%$ \\
\hline $\mathrm{Nb} 2-\mathrm{O} 6$ & $1.8592(17)$ & $1.843(3)$ & $1.854(4)$ & $0.9 \%$ & $0.6 \%$ \\
\hline Nb2-O11 & $1.8651(18)$ & $1.862(3)$ & $1.867(4)$ & $0.2 \%$ & $0.3 \%$ \\
\hline $\mathrm{Nb} 3-\mathrm{O} 2$ & $2.0655(16)$ & $2.063(3)$ & $2.063(4)$ & $0.1 \%$ & $0.0 \%$ \\
\hline $\mathrm{Nb3}-\mathrm{O} 3$ & $2.1091(17)$ & $2.110(3)$ & $2.106(5)$ & $0.0 \%$ & $-0.2 \%$ \\
\hline $\mathrm{Nb3}-\mathrm{O} 4$ & $2.1784(17)$ & $2.185(3)$ & $2.184(5)$ & $-0.3 \%$ & $0.0 \%$ \\
\hline Nb3-O7 & $1.8153(17)$ & $1.818(3)$ & $1.812(4)$ & $-0.1 \%$ & $-0.3 \%$ \\
\hline Nb3-012 & $1.8869(18)$ & $1.870(4)$ & $1.876(5)$ & $0.9 \%$ & $0.3 \%$ \\
\hline Nb3-013 & $1.8755(19)$ & $1.874(5)$ & $1.885(5)$ & $0.1 \%$ & $0.6 \%$ \\
\hline $\mathrm{Nb} 4-\mathrm{O} 2$ & $2.0207(16)$ & $2.021(3)$ & $2.022(4)$ & $0.0 \%$ & $0.0 \%$ \\
\hline $\mathrm{Nb} 4-\mathrm{O} 4$ & $2.1251(17)$ & $2.120(3)$ & $2.123(5)$ & $0.2 \%$ & $0.1 \%$ \\
\hline $\mathrm{Nb} 4-\mathrm{O} 5$ & $2.1175(17)$ & $2.103(3)$ & $2.107(5)$ & $0.7 \%$ & $0.2 \%$ \\
\hline $\mathrm{Nb4-014}$ & $1.8903(19)$ & $1.878(4)$ & $1.868(5)$ & $0.7 \%$ & $-0.5 \%$ \\
\hline Nb4-015 & $1.8667(19)$ & $1.863(4)$ & $1.876(4)$ & $0.2 \%$ & $0.7 \%$ \\
\hline Nb4-O8 & $1.8695(17)$ & $1.876(3)$ & $1.869(4)$ & $-0.3 \%$ & $-0.4 \%$ \\
\hline
\end{tabular}


Table S6.2. $\mathrm{Nb}-\mathrm{O}-\mathrm{Nb}$ and $\mathrm{O}-\mathrm{Nb}-\mathrm{O}$ bond angles of $\mathrm{Nb}_{8} \mathrm{O}_{10}(\mathrm{OEt})_{20}$ structures.

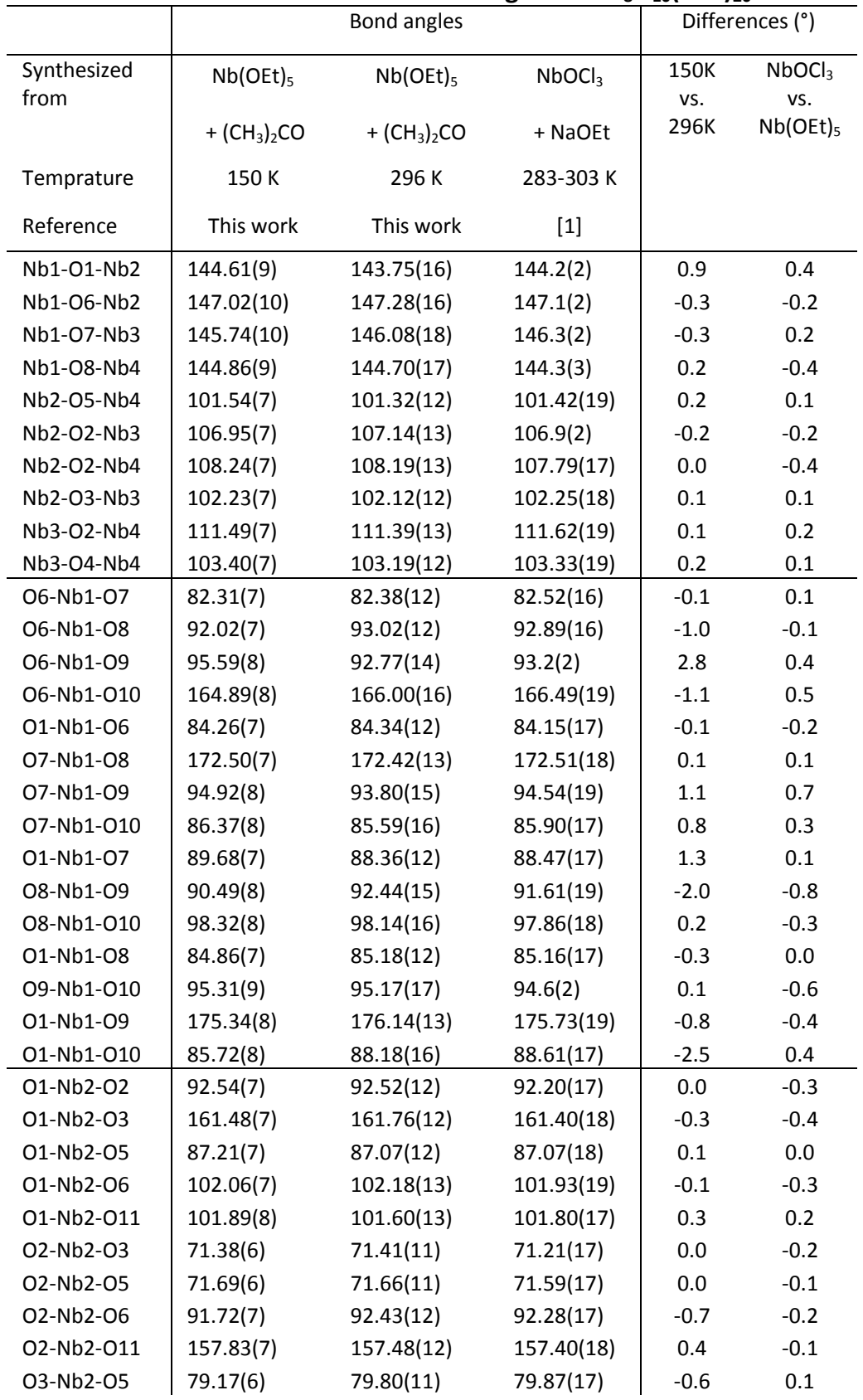


Table S6.2. Second Part.

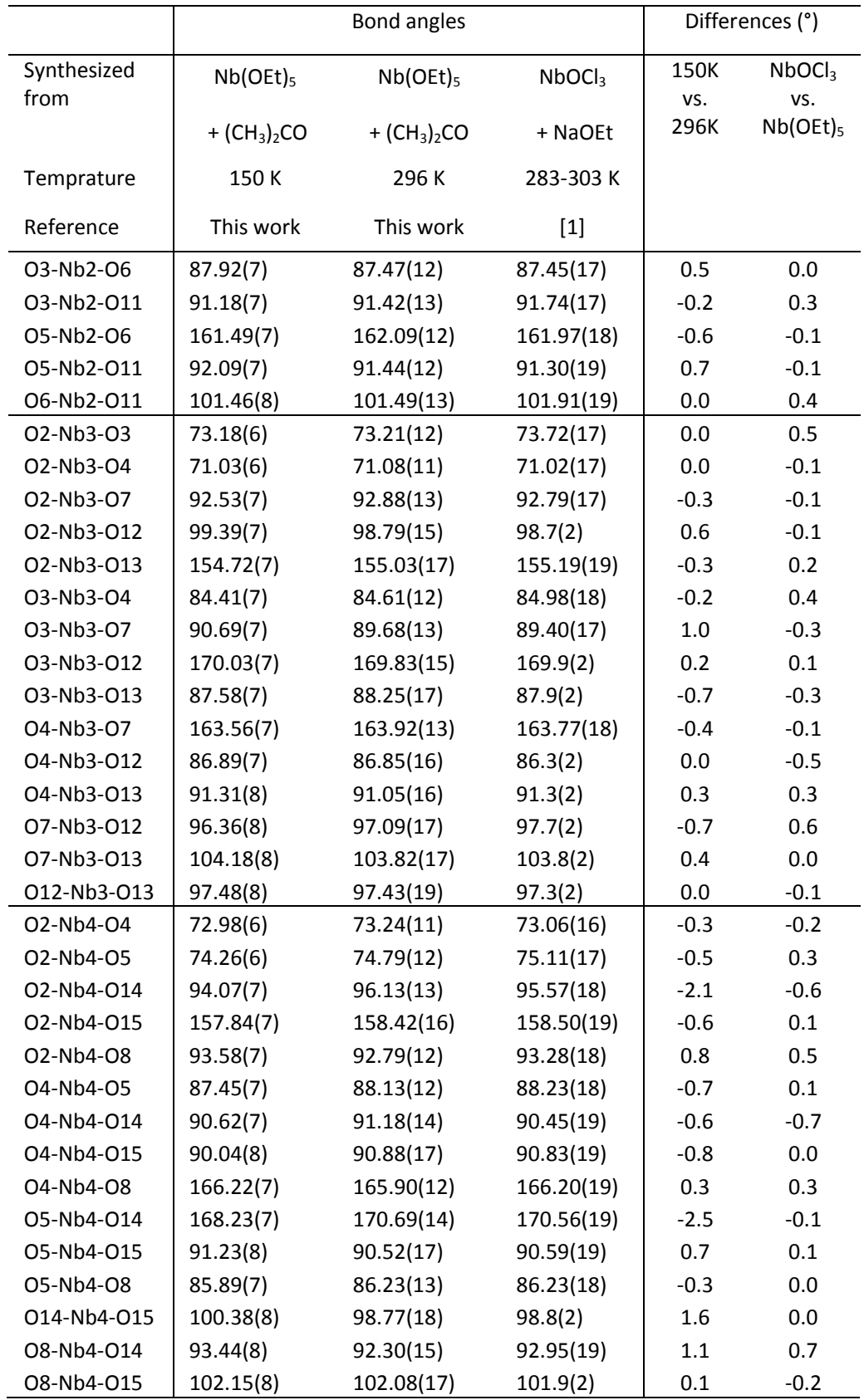




\section{S6.2. References}

1. Kessler, V.G., et al., The structure of $\mathrm{Nb}_{8} \mathrm{O}_{10}(\mathrm{OEt})_{20}$ and the nature of crystalline metal alkoxides. Russ. J. Inorg. Chem, 1991. 36(7): p. 7. 
Chapter 6 


\section{A novel malonamide bridged silsesquioxane precursor for enhanced dispersion of transition and lanthanide metal ions in hybrid silica membranes}




\subsection{Abstract}

Microporous hybrid silica membranes have superior (hydro)thermal and chemical stability. However, the permselectivities for small gases including $\mathrm{H}_{2}, \mathrm{CO}_{2}, \mathrm{~N}_{2}$ and $\mathrm{CH}_{4}$ is limited due to the limited differences between the kinetic diameters of these gases. By incorporating metal ions, such as $\mathrm{Ce}^{4+}$ and $\mathrm{Ni}^{2+}$, the affinity and therefore the selectivity of these membranes towards particular gases may be altered. To promote the dispersion of metal ions within the hybrid silica matrix, the silica precursor $N, N, N^{\prime}, N^{\prime}-$ tetrakis-(3-(triethoxysilyl)-propyl)-malonamide (TTPMA) was synthesized successfully. The malonamide ligands clearly coordinated the $\mathrm{Ce}^{4+}$ and $\mathrm{Ni}^{2+}$ metal centers and enhanced their dispersion. During calcination these metal centers redistributed into small nanosized grains of $\mathrm{CeO}_{2}(<5 \mathrm{~nm})$ and $\mathrm{Ni}_{2} \mathrm{O}_{3}(<15 \mathrm{~nm})$. These Ce-TTPMA and Ni-TTPMA membranes showed higher $\mathrm{H}_{2} / \mathrm{N}_{2}$ perselectivities as compared to previously reported hybrid BTESE (1,2-bis-(triethoxysilyl)ethane) membranes [1]. The TTPMA-precursor was found suitable for membrane separation and can be a promising and versatile precursor for the incorporation of metal ions within hybrid silica matrices.

\subsection{Introduction}

At present over $85 \%$ of the global energy is provided by burning fossil fuels [2]. This leads to increasing amounts of $\mathrm{CO}_{2}$ into the atmosphere. Although the increasing $\mathrm{CO}_{2}$ emissions are relatively small compared to the $\mathrm{CO}_{2}$ involved in the natural carbon fluxes, the $\mathrm{CO}_{2}$ concentration increase leads to a substantial climate change in a relative short time period $[2,3]$, so that we need to reduce $\mathrm{CO}_{2}$ emission. This stresses the need for separation membranes, preferentially in a post-combustion scheme in which $\mathrm{CO}_{2}$ is separated from flue gas $\mathrm{N}_{2}$ [2]. Post-combustion setups can be implemented most easily in our current power plants. $\mathrm{CO}_{2}$ separating membranes are also useful for conversion of $\mathrm{H}_{2}$ and $\mathrm{CO}_{2}$ into synthetic fuels such as $\mathrm{CH}_{3} \mathrm{OH}$ and dimethyl ether, that can be stored more easily than $\mathrm{H}_{2}$ and $\mathrm{CO}_{2}[4]$.

Nevertheless, separation of $\mathrm{CO}_{2}$ and $\mathrm{N}_{2}$ is difficult, since these gases have a similar kinetic diameter of 3.30 and $3.64 \AA$, respectively [3]. Most of the membranes that have been developed so far do not reach the required $\mathrm{CO}_{2} / \mathrm{N}_{2}$ selectivity of 200 to make them economically feasible [5]. The 
membranes that meet these requirements are based on affinity with $\mathrm{CO}_{2}$, such as glycine-sodium glycerol, where performance relies on the presence of moisture [6]. Currently, solution based adsorption on amines is still the most commonly applied technique to sequester $\mathrm{CO}_{2}$, but regeneration requires a lot of energy, the amine loading capacity is limited due to corrosion problems, and the amines degrade too fast $[2,4]$.

As alternative to post-combustion schemes, $\mathrm{CO}_{2}$ can also be separated from $\mathrm{H}_{2}$ after gasification in a pre-combustion setup [2, 7]. The difference in kinetic diameter between $\mathrm{H}_{2}$ and $\mathrm{CO}_{2}$ is slightly larger (2.89 versus $3.30 \AA$ ) and these gases can be separated by size exclusion in silica membranes with a permselectivity of 70 [8]. The selectivity of silica membranes towards $\mathrm{H}_{2}$ was increased with one order of magnitude by doping them with cobalt or nickel oxide $[9,10]$. These metal centres increased the surface adsorption and thereby the surface diffusion rate of $\mathrm{H}_{2}$ on the pore walls. Although inorganic size exclusion membranes will never exceed the permselectivity of palladium membranes, they are less expensive, catalytically inactive and do not suffer from hydrogen enbrittlement and surface poisoning $[7,11]$.

The $\mathrm{CO}_{2} / \mathrm{N}_{2}$ permselectivity of size exclusion membranes such as sol-gel derived silica membranes can be altered by increasing their affinity towards $\mathrm{CO}_{2}[10,12-15]$. However, $\mathrm{CO}_{2}$ is known to bind irreversibly with a large variety of metal oxides and the choice of a proper adsorbent is difficult [16, 17]. $\mathrm{CO}_{2}$ has a particularly strong affinity towards nucleophilic, electron donating species due to its electron-deficient carbonyl atom, and chemisorbs strongly and irreversibly onto basic sites of alkaline earth metal oxides $[18,19]$. This explains the poor mobility of $\mathrm{CO}_{2}$ in mesoporous magnesia doped $\gamma$ alumina [20]. By doping $\gamma$-alumina with magnesia the amount of $\mathrm{CO}_{2}$ adsorbed on the surface did not increase. However, the mobility of adsorbed $\mathrm{CO}_{2}$ species decreased. The authors suggested that this was caused by the replacement of weakly basic sites by strongly basic sites. On the other hand, the amphoteric character of $\mathrm{CO}_{2}$ allows chemisorption on acidic sites as well [21], which explains the low permeance of $\mathrm{CO}_{2}$ in microporous niobia doped silica [14]. Ceria may be a good alternative, since it is a weaker nucleophile as compared to earth alkaline oxides [22], while Fourier Transform Infrared (FTIR) experiments revealed substantial affinity of $\mathrm{CO}_{2}$ on a ceria surface $[23,24]$. 
Dispersion of metal centres on atomic scale into a microporous silica or hybrid silica network is not a straightforward task: the metal oxide that is introduced, either in the form of a metal salt or a metal alkoxide, tends to phase separate while the silica matrix forms. Metal salts, e.g. metal nitrates, have limited solubility within the alcoholic environments in which the silica sol is formed and these salts tend to precipitate while the sol is drying [10]. Metal alkoxides dissolve well in apolar silicates, but they are much more reactive towards hydrolysis and condensation than silica precursors, generally resulting in the formation of dense metal oxide clusters within the silica sol [25-27]. The reactivity of these alkoxides can be tempered by chelating ligands such as acetylacetone and they clearly retard the gelation in the presence of water [28-30]. It has been debated whether the reactivity is reduced by reduction of the nucleophilic character, or that the chelating ligands shield the surface from reactive species, i.e. a steric hindrance effect $[26,30]$. In the latter case, the introduction of chelating ligands would not help to increase the degree of dispersion of the metal oxide in a silica matrix, since they would be shielding the metal centre from silicon species as well.

A more elegant solution was proposed by Rupp et al. [31-33], who synthesized a triethoxysilylpropyl adduct to acetylacetone and subsequently coupled this molecule to a titanium, zirconium or aluminium alkoxide. This approach solved two complications simultaneously: (1) it connects the metal alkoxide precursors to the silica precursors before the sol-gel process, thereby increasing the dispersion of the metal alkoxides within the glassy silica matrix; (2) it tempers and shields the reactive metal centre in the metal alkoxide precursor, thereby reducing its tendency to form a condensed metal oxide [31]. The latter possibility was supported by XRD data that revealed a strong reduction of the anatase and rutile grain sizes [31], and SAXS data that revealed a limited metal oxide size cluster size with aluminium alkoxides [33].

In the present work we used a similar approach. However, we chose to form a bridged silsesquioxane precursor with four triethoxysilyl-propyl groups attached to a single malonamide chelating group. As compared to pendant silsesquioxanes, bridged silsesquioxanes exhibit much higher mechanical strength and fracture resistance due to enhanced connectivity and the absence of dangling carbon substituents [34-36]. A bridged silsesquioxane that consists of a malonamide chelating group was formed through the formation of amide bonds between malonyl chloride and two 
bis-(3-(triethoxysilyl)-propyl)-amine molecules. The synthesis was performed via a base catalyzed (by using a tertiary amine) $\mathrm{N}$-acylation of malonyl chloride on $\mathrm{N}$-substituted amines in dichloromethane at temperatures below $T=5^{\circ} \mathrm{C}$ as reported before [37, 38] (Scheme 7.1).
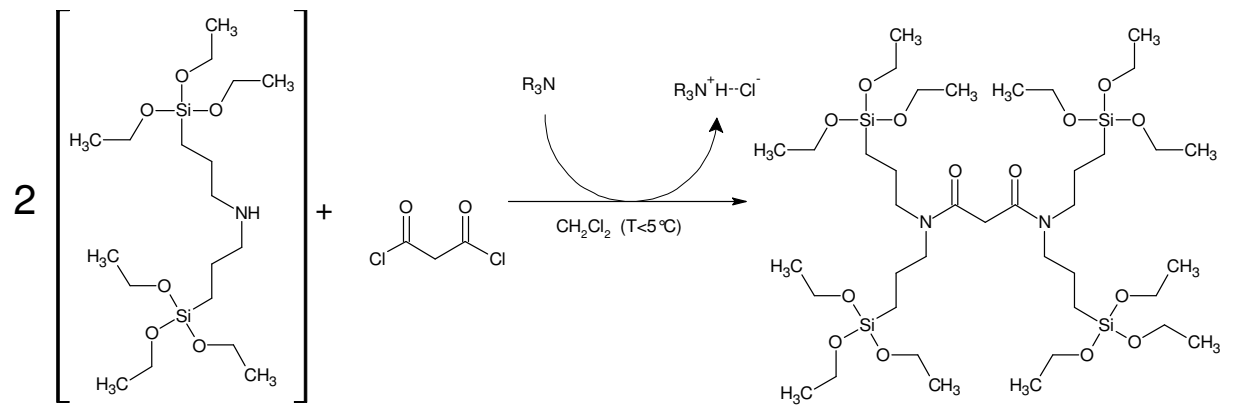

Scheme 7.1 Synthesis of $\mathrm{N}, \mathrm{N}, \mathrm{N}^{\prime}, \mathrm{N}^{\prime}$-tetrakis-(3-(triethoxysilyl)-propyl)-malonamide (TTPMA)

Malonamides are strong chelating units. Through the formation of an enolic bidentate ligand they can easily form complex bonds with a large variety of metals cations, including lanthanides and actinides [39-46]. Since a large variety of metal cations can thus be incorporated into a hybrid silica matrix, the approach is very versatile and its affinity towards certain probe molecules can be controlled by the choice of metal(s). In this study, we doped the TTPMA matrix with cerium (IV) isopropoxide isopropanol adduct and nickel nitrate hexahydrate in an attempt to enhance the affinity towards carbon dioxide and $\mathrm{H}_{2}$, respectively.

\subsection{Experimental section}

\subsubsection{Synthesis of $N, N, N^{\prime}, N^{\prime}$-tetrakis-(3-(triethoxysilyl)- propyl)-malonamide (TTPMA)}

The synthesis was performed inside a glovebox under nitrogen atmosphere. Before starting the synthesis dichloromethane (BOOM, 99\%) triethylamine (Sigma aldrich, 99\%), and dimethylsulfoxide (Sigma Aldrich 99\%) were dried over anhydrous sodium sulphate (Sigma Aldrich, 99\%) and dichloromethane was cooled down to $T=-5^{\circ} \mathrm{C}$ for $24 \mathrm{~h}$ in a freezer. Then, $30 \mathrm{mmole}$ of bis-(3(triethoxysilyl)-propyl)-amine (ABCR chemicals, 97\%) was dissolved in 183 
$\mathrm{mL}$ of dichloromethane inside a $250 \mathrm{~mL}$ Erlenmeyer flask. Subsequently, 30 mmole of triethylamine was added to the solution. In a dropping funnel, a $10 \%$ excess (16.5 mmole) of malonylchloride (Sigma Aldrich, 97\%) was dissolved in $48.4 \mathrm{~mL}$ of dichloromethane, which was added dropwise to the solution in the Erlenmeyer flask in $30 \mathrm{~min}$. Please note that malonyl chloride is rather reactive and may decompose with the formation of hydrochloric acid when the temperature increases, thus after use the bottle was immediately returned to the fridge. After addition, the Erlenmeyer flask was stirred for 7 days at room temperature.

While stirring the Erlenmeyer flask inside an oil bath at $T=20^{\circ} \mathrm{C}$, the dichloromethane was distilled using a Vacuumbrand vacuum pump that automatically adapts its pressure to the solvent pressure. Then $150 \mathrm{~mL}$ of hexane was used to extract TTPMA from the remnant slurry. The triethylammonium chloride that was formed during the reaction was removed by filtration using a $2.7 \mu \mathrm{m}$ filter paper (Whatman, grade 50) over a Büchner funnel. The Erlenmeyer flask and Büchner funnel were washed twice with $50 \mathrm{~mL}$ hexane. The filtered hexane solution was washed once with dimethylsulfoxide to remove remnant ammonium byproducts. From the hexane solution the hexane was removed by vacuum distillation at room temperature. Afterwards the dimethylsulfoxide was removed by vacuum distillation at $T=80^{\circ} \mathrm{C}$.

\subsubsection{NMR-measurements}

All measurements were done on a Bruker Ascend-400 spectrometer operating at $400,13 \mathrm{MHz}$ for ${ }^{1} \mathrm{H}$ - and $100,61 \mathrm{MHz}$ for ${ }^{13} \mathrm{C}$, using a $5 \mathrm{~mm}$ gradient-probe. Concerning the 1D NMR-experiments 16, 4096 and 2048 scans were accumulated for ${ }^{1} \mathrm{H}-\mathrm{NMR}$, inverse gated ${ }^{1} \mathrm{H}$-decoupled ${ }^{13} \mathrm{C}$-NMR and ${ }^{13}$ C-DEPT-135-NMR (Distortionless Enhancements by Polarization Transfer) experiments, respectively. The 1D experiments were performed following the pulse program sequences: zg30, zgig30 and deptsp135 as described in Bruker's pulse program catalogue [47].

A homonuclear ${ }^{1} \mathrm{H}-{ }^{1} \mathrm{H}$ correlation spectrum was obtained with 4 scans by using a magnitude mode gradient enhanced 2D-COSY (COrrelation SpectroscopY) experiment. The direct $\mathrm{H}-\mathrm{C}$ couplings were measured by an

${ }^{1} J_{\mathrm{H}-\mathrm{C}} \mathrm{Scalar}$ correlation spectrum, which was acquired with a phase-sensitive gradient selected HSQC pulse sequence (Heteronuclear Single Quantum 
Coherence) [48]. The measurement was acquired with 32 scans and a delay $1 /\left(4 \cdot{ }^{1} J_{C-H}\right)=1.72 \mathrm{~ms}$, which corresponds to a mean ${ }^{1} J_{C-H}=145 \mathrm{~Hz}$. A heteronuclear ${ }^{1} \mathrm{H}-{ }^{13} \mathrm{C}$ long range scalar correlation spectrum was obtained using gradient selected $\mathrm{HMBC}$ pulse sequence (Heteronuclear Multiple Bond Correlation) $[49,50]$. Several one bond $\mathrm{H}-\mathrm{C}$ correlation peaks in the 2D spectrum appeared as doublets, since the direct ${ }^{1} J_{\mathrm{C}-\mathrm{H}}$-bond correlations were not completely suppressed by the low-pass J-filter. The measurement was acquired with 64 scans, a delay $1 /\left(2 \cdot{ }^{1} J_{-H}\right)=3.45 \mathrm{~ms}$ and a delay $\tau=$ $0.05 \mathrm{~s}$ for long range couplings. The COSY, HSQC and HMBC experiments were performed following the pulse program sequences: cosygpppqf, hsqcetgp and hmbcgplpndqf [47].

\subsubsection{Sol preparation and DLS measurements}

Before preparing the Ce-TTPMA sol a stock-solution was prepared: At first approximately 3 mmole of $\mathrm{Ce}\left(\mathrm{O}^{\prime} \mathrm{Pr}\right)_{4}{ }^{\mathrm{i}} \mathrm{PrOH}$ (CeTiP; ABCR Chemicals, 31.5$32.4 \%$ Ce weight) was weighed accurately in a $50 \mathrm{~mL}$ polypropylene vessel. Then, a stoichiometric amount of TTPMA was added to yield a [Ce]/[Si] ratio of $1: 9$, which corresponded to $[\mathrm{Ce}] /[\mathrm{TTPMA}]=1 / 2.25$. This mixture was diluted in $15 \mathrm{~mL}$ of isopropanol, yielding a solution with a density of 1.28 $\mathrm{g} / \mathrm{mL}$, which corresponded to $[$ TTPMA] $=0.295 \mathrm{~mol} / \mathrm{L}$ and $[\mathrm{Ce}]=0.131$ mole/L. After stirring the solution for $24 \mathrm{~h}$ at room temperature, it was further diluted to yield a $[O R]=1.8 \mathrm{~mole} / \mathrm{L}$, where $[\mathrm{OR}]$ represents the concentration of reactive alkoxy groups, i.e. the sum of both ethoxy and isopropoxy groups from TTPMA and cerium isopropoxide, respectively. Then, $30 \mathrm{~mL}$ of an acidic solution of water and nitric acid in isopropanol $\left(\left[\mathrm{H}_{2} \mathrm{O}\right]=1.8 \mathrm{~mol} / \mathrm{L} ;\left[\mathrm{HNO}_{3}\right]=0.06 \mathrm{~mol} / \mathrm{L}\right)$ to $30 \mathrm{~mL}$ of diluted stock solution to yield a solution with the following characteristics: $[O R]=0.9 \mathrm{~mole} / \mathrm{L}$, $\left[\mathrm{H}_{2} \mathrm{O}\right] /[\mathrm{OR}]=1,\left[\mathrm{HNO}_{3}\right] /[\mathrm{OR}]=1 / 30,[\mathrm{TTPMA}]=65.4 \mathrm{mmole} / \mathrm{L}$ and $[\mathrm{Ce}]=$ $29.1 \mathrm{mmole} / \mathrm{L}$. Finally, sol particles were formed by heating the solution for $1 \mathrm{~h}$ at $T=60^{\circ} \mathrm{C}$ in a closed $100 \mathrm{~mL}$ round bottom flask. For membrane preparation the $[\mathrm{OR}]=0.9 \mathrm{~mol} / \mathrm{L} \mathrm{Ce}$-TTPMA sol was diluted 6 times by volume before coating it on the mesoporous support.

At first, for the Ni-TTPMA sol, a $\mathrm{Ni}^{2+}$ stock solution was prepared: Approximately $10 \mathrm{~g}$ of $\mathrm{Ni}\left(\mathrm{NO}_{3}\right)_{2} \cdot 6 \mathrm{H}_{2} \mathrm{O}$ was weighed accurately in a $50 \mathrm{~mL}$ glass bottle and dissolved in approximately $46 \mathrm{~mL}$ of ethanol which was weighed afterwards. This yielded a stock solution with $[\mathrm{Ni}]=0.691 \mathrm{~mole} / \mathrm{L}$ and $\rho=0.922 \mathrm{~g} / \mathrm{mL}$. The Ni stock solution and TTPMA were dissolved in 
ethanol to yield diluted solutions with $[\mathrm{OR}]=1.8,2.4,3.0$ and $3.6 \mathrm{~mole} / \mathrm{L}$, where [OR] is defined as the sum of both ethoxy groups of TTPMA and nitrate groups from nickel nitrate, i.e. $[\mathrm{OR}]=\left[\mathrm{NO}_{3}{ }^{-}\right]+[-\mathrm{OEt}]$. After stirring these solutions for $2 \mathrm{~h}$ at room temperature, acidic solutions of nitric acid and water were added dropwise to yield 8 different sols with two different acid ratios $\left[\mathrm{H}^{+}\right] /[\mathrm{OR}]=1 / 30$ and $1 / 15$, and four different concentrations $[\mathrm{OR}]=0.9,1.2,1.5$ and 1.8 , respectively. The water ratio $\left[\mathrm{H}_{2} \mathrm{O}\right] /[\mathrm{OR}]=1$ (including crystal water from the $\mathrm{Ni}$-stock) and complexing ratio $[\mathrm{Ni}] /[\mathrm{TTPMA}]=1$ were held constant for all sols. The sols were heated in closed glass round bottom flasks for $1 \mathrm{~h}$ at $T=60^{\circ} \mathrm{C}$ while stirring. Before the particle size was analyzed by dynamic light scattering (DLS), all sols were diluted to yield an overall concentration $[O R]=0.9 \mathrm{~mol} / \mathrm{L}([\mathrm{TTPMA}]=64.3$ $\mathrm{mmole} / \mathrm{L}$ ). The sol with $[\mathrm{OR}]=1.2 \mathrm{~mol} / \mathrm{L}$ (during synthesis) and $\left[\mathrm{H}^{+}\right] /[\mathrm{OR}]=$ $1 / 15$ was diluted to $[O R]=0.9 \mathrm{~mol} / \mathrm{L}$ and used for membrane fabrication.

After 75 vol\% dilution in ethanol, the hydrodynamic diameter of freshly prepared Ce-TTPMA and Ta-TTPMA sols were determined by dynamic light scattering (DLS) technique using a Malvern Zetasizer Nano ZS at $25^{\circ} \mathrm{C}$. The hydrodynamic diameter of the sol particles was determined from Brownian motion of particles as defined by the translational diffusion coefficient $D$ in the ethanol solvent.

\subsubsection{FTIR measurements}

The Fourier Transform Infra Red (FTIR) measurements were performed by using a Brucker Tensor 27 equipped with a $\mathrm{KBr}$ beam splitter, a Pike Gladi ATR diamond attenuated total reflectance (ATR) unit and a liquid nitrogen cooled MCT broadband detector. The FTIR spectra were recorded in 5 min, with a resolution of $1 \mathrm{~cm}^{-1}$. The Fourier transform (FT) was performed with 9480 phase interferogram points and a Blackman-Harris 3-term apodization function.

FTIR measurements were used as a tool to measure the extent of complexation after aging TTPMA with $\mathrm{Ce}\left(\mathrm{O}^{\mathrm{i}} \mathrm{Pr}\right)_{4}{ }^{\mathrm{i}} \mathrm{PrOH}$ (CeTiP) in different ratios. Therefore, TTPMA and CeTiP, both viscous liquids, were mixed inside a glovebox for $24 \mathrm{~h}$ in the absence of any solvent with complexation ratios $r_{\mathrm{C}}=[\mathrm{Ce}] /[\mathrm{TTPMA}]$ is $\frac{1}{4}, \frac{1}{3}, \frac{1}{2}, 1$ and 2 , respectively. Since the FTIR equipment was located outside the glovebox, a septum was mounted on the ATR 
sample unit to minimize the contact with atmospheric water and then the sample was loaded with a syringe.

Complexation of Ni-TTPMA mixtures were also characterized by FTIR. Therefore, the same $0.691 \mathrm{~mol} / \mathrm{L} \mathrm{Ni}$ stock solution in ethanol as described in section 2.3 was used. Then, TTPMA and the nickel stock solution were mixed in different molar ratios, with $r_{\mathrm{C}}=[\mathrm{Ni}] /[\mathrm{TTPMA}]$ is $\frac{1}{4}, \frac{1}{2}, 1$ and 2 , respectively and diluted with ethanol to yield a concentration [TTPMA] = $0.2 \mathrm{~mole} / \mathrm{L}$ for all mixtures. These mixtures were aged for $2 \mathrm{~h}$ before performing the FTIR measurements. Moreover, FTIR measurements were performed on the Ni-TTPMA sample with $r_{\mathrm{C}}=1$ at several time intervals of $15 \mathrm{~min}$, with a total duration between $15 \mathrm{~min}$ and $24 \mathrm{~h}$. For the sake of comparison the same experiment was repeated using $N, N, N^{\prime}, N^{\prime}$-tetra- $n$ propyl-malonamide (ABCR-chemicals, TPMA) instead of TTPMA under the same conditions as in the $r_{C}=1$ sample of TTPMA.

For further characterization the undiluted Ce-TTPMA sol ([OR] $=0.9$ mole/L, $\left.\left[\mathrm{H}^{+}\right] /[\mathrm{OR}]=1 / 30\right)$ and Ni-TTPMA $\left([\mathrm{OR}]=0.9 \mathrm{M},\left[\mathrm{H}^{+}\right] /[\mathrm{OR}]=1 / 15\right)$ were spincast on plasma oxidized Si-substrates with a Laurell WS- 400B-6NPP-Lite spincoater at $3000 \mathrm{rpm}$ for $1 \mathrm{~min}$. For both Ni-TTPMA and Ce-TTPMA sol two substrates were coated: one was dried for $24 \mathrm{~h}$ at $T=60^{\circ} \mathrm{C}$ and the other one was dried for $24 \mathrm{~h}$ at $\mathrm{T}=60^{\circ} \mathrm{C}$ and subsequently calcined at $T=250^{\circ} \mathrm{C}$. These spincast films were used for FTIR and XPS characterization.

\subsubsection{TEM, SEM and XPS measurements}

For the Transmission electron microscopy (TEM) measurements carbon TEM copper grids (CF200-Cu, Electron Microscopy Sciences) were dipcoated in Ni-TTPMA $\left([\mathrm{OR}]=0.9 \mathrm{~mol} / \mathrm{L},\left[\mathrm{H}^{+}\right] /[\mathrm{OR}]=1 / 15\right)$ and undiluted Ce-TTPMA sol $\left([O R]=0.9 \mathrm{~mol} / \mathrm{L},\left[\mathrm{H}^{+}\right] /[\mathrm{OR}]=1 / 30\right)$. Then, the as-prepared films were dried at $60^{\circ} \mathrm{C}$ for $24 \mathrm{~h}$ in a furnace. Subsequently, these samples were analyzed with a Philips CM300STFEG TEM at $300 \mathrm{kV}$. Samples were investigated at low magnification to find typical areas and features of interest were examined at high magnification (GATAN 2048 Ultrascan1000 CCD camera).

X-ray photoelectron spectra (XPS) were recorded with a Quantera SXM scanning XPS microprobe with a $50 \mathrm{~W}$ monochromated Al $K_{\alpha} \mathrm{X}$-ray scource of $1486.6 \mathrm{eV}$ and a spot size of $200 \mu \mathrm{m}$. These measurements were 
performed on the spincast silicon substrates as described in section 2.4. Scanning electron microscopy (SEM) was performed with a Zeiss Merlin HRSEM using $1.4 \mathrm{kV}$ acceleration voltage.

\subsubsection{Membrane fabrication and gas permeation measurements}

Disk-shaped $\alpha$-alumina supported $\gamma$-alumina membranes (pore size 3-5 nm) were prepared by a dip-coating procedure of a boehmite sol on $\alpha$-alumina supports (support thickness of $2.08 \pm 0.01 \mathrm{~mm}$ and pore diameter of $100 \mathrm{~nm}$; Pervatech B.V., The Netherlands), followed by drying and calcination, as described elsewhere [51]. A boehmite-polyvinyl alcohol (PVA) sol was prepared for dipcoating the mesoporous intermediate layer. $0.55 \mathrm{~mol}$ aluminium-tri-sec-butoxide (Merck) was added to $1150 \mathrm{~mL}$ water under vigorous stirring at $T=100^{\circ} \mathrm{C}$ and refluxing for 1 hour. At a lower temperature $\left(T=60^{\circ} \mathrm{C}\right)$ the $\mathrm{pH}$ was adjusted to 2.8 with $1 \mathrm{~mol} / \mathrm{L} \mathrm{HNO}_{3}$ and the sol was refluxed for another $21 \mathrm{~h}$ at $T=90^{\circ} \mathrm{C}$.

In a separate vessel, $3.0 \mathrm{~g}$ PVA (Merck, $\mathrm{MW}=72000 \mathrm{~g} / \mathrm{mol}$ ) was dissolved in $100 \mathrm{~g}$ of $0.05 \mathrm{M} \mathrm{HNO}_{3}$ solution while stirring it for $2 \mathrm{~h}$ at $T=80^{\circ} \mathrm{C}$. For dipcoating, $30 \mathrm{~mL}$ of boehmite sol was filtered (Schleicher\&Schuell, FP030/50 pore size $0.8 \mu \mathrm{m}$, cellulose acetate, green) and mixed with $20 \mathrm{~mL}$ of PVA solution. Then, dipcoating was performed with a Velterop DA $3960 / 02$ dipcoater (turn velocity 10 , dip velocity 0.2 ). A pore size of the $\gamma$ alumina intermediate layer of 3-5 $\mathrm{nm}$ was determined by permporometry, as described elsewhere [52]. Dip coating of a Ce- and Ni-TTPMA sol (with dip sol concentration [Si] $=0.3 \mathrm{M}$ and dipping speed of $1.4 \mathrm{~cm} / \mathrm{s}$ ) on $\gamma$ alumina membranes was done in a flow cupboard class 100, situated in a clean room class 1000 to minimize defect formation due to dust particles. The dipping procedure was performed only once to deposit a selective layer on the mesoporous support. After coating of the sol, the membranes were calcined at $250{ }^{\circ} \mathrm{C}$ for $3 \mathrm{~h}$ under nitrogen flow (99.99\% pure), applying heating and cooling rates of $0.5^{\circ} \mathrm{C} / \mathrm{min}$.

Membrane flux and selectivity were determined on a home built single gas permeation (SGP) set-up in a dead-end mode without back pressure. The membranes were sealed in a stainless steel module using Viton ${ }^{\circledR} 51414$ Orings with the separation layer exposed to the feed side. The gas permeance was measured at $200{ }^{\circ} \mathrm{C}$ in a sequence, starting with the gas 
with smallest kinetic diameter, i.e. $\mathrm{He}(0.255 \mathrm{~nm}), \mathrm{H}_{2}(0.289 \mathrm{~nm}), \mathrm{CO}_{2}(0.33$ $\mathrm{nm}), \mathrm{N}_{2}(0.364 \mathrm{~nm}), \mathrm{CH}_{4}(0.389 \mathrm{~nm})$ to $\mathrm{SF}_{6}(0.55 \mathrm{~nm})$ at $200^{\circ} \mathrm{C}$ with a pressure difference of 2 bar. Then the hydrogen flux was determined again to ensure that no change had occurred on the membrane microstructure due to permeation of the gases.

The membrane flux via SGP was derived by the following equation:

$F_{\mathrm{i}}=\frac{N_{\mathrm{i}}}{\Delta P}$

where $F_{\mathrm{i}}$ is the permeance of gas $\mathrm{i}$, defined as the ratio between the molar permeance $(N)$ of the gas going through the membrane and the applied pressure difference between the feed and permeate side of the membrane $(\Delta P)$.

\subsection{Results and discussion}

\subsection{1. synthesis and characterization of TTPMA}

The $N, N, N^{\prime}, N^{\prime}$-tetrakis-(3-(triethoxysilyl)propyl)-malonamide (TTPMA) that was formed was analysed by ${ }^{1} \mathrm{H}$ - and ${ }^{13} \mathrm{C}$-NMR spectroscopy (Figure 7.1). Concerning the assignment of the resonances the $\mathrm{CH}_{\mathrm{x}}$-moieties were divided into three groups: an alkoxy (or ethoxy) group designated by $\mathrm{A} 1$ and A2, a bridging propyl group designated by B1, B2 and B3, and a chelating group designated by $\mathrm{C} 1$ and $\mathrm{C} 2$. The assignments are clarified by the numbers in Figure 7.2. The proton resonances (Figure 7.1a, Table 7.1) of $\mathrm{CH}_{3}$ (designated by $\mathrm{A} 1$ ) and $\mathrm{CH}_{2}$ (designated by $\mathrm{A} 2$ ) remained the same position with respect to the reactant bis-(3-triethoxysilylpropyl)-amine (BTESPA). These atoms are not close to the nitrogen atoms that were involved in the reaction and therefore these resonances were unaffected by the formation of the amide bonds. The difference in the assigned chemical shifts between BTESPA and TTPMA was most pronounced for the $\mathrm{CH}_{2}$-group next to the amine/amide bond designated by $\mathrm{B} 3$, where the resonating proton became more shielded $(0.23 \mathrm{ppm})$ by the formation of an amide. 
The assigned proton resonances were also compared with the assignments of $N, N, N^{\prime}, N^{\prime}$-tetra- $n$-propyl-malonamide (TPMA) [53] and are given in Table 7.1. For TPMA two slightly different chemical shifts were reported for the methylene groups, due to their geographical orientation with respect to the carbonyl group. The protons of the methylene group that are cis-oriented with respect to the carbonyl oxygen atom designated by $\mathrm{B} 3 \mathrm{a}$ are slightly more shielded by the $\pi$-electrons of the carbonyl group in comparison with the trans-oriented methylene group (B3b), causing a difference in chemical shift of 0.03 ppm (Table 7.1). The proton NMR spectrum of TTPMA (Figure 7.1a) reveals a multiplet at $\delta=3.24 \mathrm{ppm}$ that consists of overlapping resonances of cis and trans oriented $\mathrm{CH}_{2}$ groups. It is not possible to deconvolute them solely by $\mathrm{H}-\mathrm{NMR}$, although these resonances can be distinghuised by the ${ }^{1} \mathrm{H},{ }^{13} \mathrm{C}-\mathrm{HSQC}$-spectrum (Figure 7.1d) as discussed below. This multiplet was found at a sligthly lower chemical shift (0.07 ppm) with respect to TPMA due to electron repulsion of the silicon atom. The differences between the proton reconances of the propyl groups of TTPMA and TPMA became more pronounced when going from the methylene group B3 towards the methylene group B1 next to the silicon atom. Finally, the H-NMR spectrum consisted of a sharp singlet at $\delta=3.38$ ppm that was associated with the $\mathrm{CH}_{2}$-group of the malonyl segment in between the carbonyl groups that are designated by $\mathrm{C} 2$.

The H-NMR assignments were supported by the cross correlation peaks that were found in the ${ }^{1} \mathrm{H},{ }^{1} \mathrm{H}$-COSY spectrum. Correlation between the methyl and methylene moieties of the ethoxide groups were confirmed by the cross correlation peaks between the $A 1$ and $A 2$ resonances as indicated by the purple dotted lines Figure 7.1c. The correlations between B1 and B2, and B2 and B3 as clarified by the blue lines in Figure 7.1c correspond to the vicinal J-couplings between the neighbouring methylene groups of the propylene linkage.

The resonances of the $\mathrm{B} 3 \mathrm{a}$ and $\mathrm{B} 3 \mathrm{~b}$ methylene groups that were cis- and trans-coordinated with respect to the carbonyl group became more distinct in the ${ }^{1} \mathrm{H},{ }^{13} \mathrm{C}$-HSQC-spectrum (Figure 7.1d), since the differences between the ${ }^{13} \mathrm{C}$ chemical shifts were more substantial $(2.32 \mathrm{ppm})$. The resonances belonging to the methylene groups next to the $\mathrm{B} 3 \mathrm{a}$ and $\mathrm{B} 3 \mathrm{~b}$ methylene groups, designated by $\mathrm{B} 2 \mathrm{a}$ and $\mathrm{B} 2 \mathrm{~b}$, respectively, were also well separated in the ${ }^{1} \mathrm{H},{ }^{13} \mathrm{C}-\mathrm{HSQC}$ spectrum. Contrary to the TPMA spectrum, the ${ }^{1} \mathrm{H},{ }^{13} \mathrm{C}$ HSQC did not reveal a clear difference between B1a and B1b resonances. 
Most likely, these chemical shifts were predominantly determined by electron repulsion of silicon. In comparison, the carbonyl group had only a minor effect which was insufficient to separate the resonances. Due to the absence of silicon in the case of TPMA, the influence of the carbonyl group was more pronounced as compared to TTPMA.

The negative signals in the ${ }^{13} \mathrm{C}$-DEPT- $135^{\circ}$ spectrum (Figure 7.1b) confirm that the resonances $\mathrm{A} 2, \mathrm{~B} 1, \mathrm{~B} 2 \mathrm{a}, \mathrm{B} 2 \mathrm{~b}, \mathrm{~B} 3 \mathrm{a}, \mathrm{B} 3 \mathrm{~b}$ and $\mathrm{C} 2$ can be associated with $\mathrm{CH}_{2}$-groups. The positive signal of resonance $A 1$ was consistent with a $\mathrm{CH}_{3}$-group. Each assigned propylene bridging resconance, i.e. B1, B2a, B2b, $\mathrm{B} 3 \mathrm{a}$ and $\mathrm{B} 3 \mathrm{~b}$, seemed to be split into at least two different resconances in the ${ }^{13} \mathrm{C}$-DEPT- $135^{\circ}$ spectrum. This may indicate the existence of several conformations of the TTPMA molecule.

Direct ${ }^{1} J-{ }^{1} \mathrm{H}-{ }^{13} \mathrm{C}$ couplings were not completely suppressed by the low-pass $J$-filter and consequently the resonances were split into doublets on the proton axis in the HMBC-spectrum (Figure 7.1f). In the image we clarify the position of these doublets with dotted boxes that connect them with each other. Cross-correlation peaks were observed for the $\mathrm{CH}_{\mathrm{x}}$-groups of the ethoxide groups, i.e., between $A 1$ and $A 2$, and between the methylene groups of the propylene linkage, i.e., B1-B2-B3, which are in good aggreement with the cross-correlation observed in the COSY spectrum as described above. In addition, the HMBC confirms a coupling between the methylene resonance (C2) and a carbon resonance at $166.8 \mathrm{ppm}$ that was associated with the carbonyl-goup (assigned as C1) of the malonamide unit (Figure 7.1e,f). Interestingly, a cross-correlation between the carbonyl group carbon atom (C1) and the methylene protons next to the nitrogen atom of the propyl-linkage, i.e., B3a and B3b, was also observed. These long range couplings were also observed for other types of amines and they are a strong evidence that both resonances were connected through the amide bond [54-56].

Nevertheless, the ${ }^{1} \mathrm{H},{ }^{13} \mathrm{C}-\mathrm{HMBC}$ and the inverse gated ${ }^{13} \mathrm{C}$-NMR reveal the presence of some other carbonyl moieties that most likely belong to byproducts of the reaction. These impurities were divided in two sets of correlated resonances. Set 1 contains two carbonyl moieties at 196.6 and $166.8 \mathrm{ppm}$, respectively, that were coupled with a $\mathrm{CH}_{2}$-group $\left({ }^{13} \mathrm{C}\right.$-DEPT$135^{\circ}$ spectrum, Figure 7.1b) with a proton resonance of $3.62 \mathrm{ppm}$ and carbon resonance of $47.08 \mathrm{ppm}$. The resonance at $166.8 \mathrm{ppm}$ corresponds 
with the resonance of the carbonyl-moiety of TTPMA and was most likely also attached to an $-\mathrm{NR}_{2}$ group that originated from BTESPA.

The other resonance at $196.6 \mathrm{ppm}$ could neither be associated with enolic malonamide (due to the low chemical shift of the $\mathrm{CH}_{\mathrm{x}}$-moiety), nor with an aldehydic carbonyl group (due to the absence of a proton shift at 9-10 ppm), and its chemical shift is too high to be associated with an ester, amide or carbonyl chloride [57]. Most likely, it was attached to another carbon atom. Set 2 contained two carbonyl resonances at 165.2 and 196.6 ppm that were coupled with a $\mathrm{CH}_{\mathrm{x}}$-moiety with a proton resonance at 5.13 ppm, and, according to the HSQC-spectrum (Figure 7.1d), a carbon resonance at $62.67 \mathrm{ppm}$. The chemical shifts of the $\mathrm{CH}_{\mathrm{x}}$-moiety were outside the range that is associated with $\mathrm{CH}_{3}$-groups, or with an unsaturated $\mathrm{C}=\mathrm{C}$-bond as in the case of enolic $\beta$-diketones [57].

Moreover, the positive signal in the ${ }^{13} \mathrm{C}$-DEPT $135^{\circ}$-spectrum (Figure 7.1b) eliminated the possibility to assign these resconances to a $\mathrm{CH}_{2}$-group. Most likely, this moiety consists of an (-CHX-) group in between two carbonyl moeities, where $X$ may be a chlorine atom. Similar shifts (61.45 and 4.82 $\mathrm{ppm})$ as found here were reported for the (-CHCl-) group of ethyl 2-chloro3-oxobutyrate [58]. The $\mathrm{X}$-atom may also correspond to other substituents $\left(-\mathrm{NR}_{2}\right.$ or $\left.-\mathrm{OR}\right)$. However, the ${ }^{1} \mathrm{H},{ }^{1} \mathrm{H}$-COSY spectrum did not reveal an additional set of correlated peaks that belongs to either $-\mathrm{NR}_{2}$ or $-\mathrm{OR}$ substituents. If one of them would have been attached to this carbon atom, then their chemical nature would have been substantially different.

The resonance at $196.6 \mathrm{ppm}$ could not be discriminated from a similar resonance in set 1 in the inverse gated ${ }^{13} \mathrm{C}-\mathrm{NMR}$ spectrum, see Figure $7.1 \mathrm{~g}$. Thus, it is most likely that both resonances are associated with the same carbonyl atom despite the absence of other couplings between both sets of coupled resconances in the HMBC-spectrum (Figure 7.1e,f). This resonance was reasonably close to a similar carbonyl moiety in diethyl-3-oxoglutarate (195.6 ppm) [59], which made the formation of 2-chloro-pentane-1,3,5trione a plausible senario. In summary, the best explanation for the additional resonances that were found in the NMR-spectra is that 2-chloro3-oxo- $N, N, N^{\prime}, N^{\prime}$-tetrakis-(3-(triethoxysilyl)-propyl)-pentanediamide was formed as a by-product.

A similar phenomenon was observed for $\mathrm{N}$-hydroxylsuccinimidyl activated malonic acid half esters that self-condense via a base catalyzed Claisen 
condensation [60], also resulting in the formation of a pentane-1,3,5-trione. The additional resonances that were assigned to this by-product are listed in Table 7.2. Most of the proton and carbon resonances corresponding to the propylene linkages strongly overlap with similar resonances of TTPMA. Only the $\mathrm{CH}_{2}$-group of the propylene linkages closest to the (- $\mathrm{CHCl}$-) linkage that were assigned as R6a and R6b deviated slightly from the B3a and B3b resonances that were assigned to TTPMA.

Table 7.1. List of ${ }^{1} \mathrm{H}$ - and ${ }^{13} \mathrm{C}$-NMR resonances assigned to the TTPMA-spectra.

\begin{tabular}{|c|c|c|c|c|c|c|c|c|c|c|c|}
\hline \multicolumn{2}{|c|}{ Assignment } & A1 & A2 & B1a & B1b & B2a & B2b & B3a & B3b & C1 & C2 \\
\hline \multicolumn{2}{|c|}{ Group } & $\mathrm{CH}_{3}$ & $\mathrm{CH}_{2}$ & $\mathrm{CH}_{2}$ & $\mathrm{CH}_{2}$ & $\mathrm{CH}_{2}$ & $\mathrm{CH}_{2}$ & $\mathrm{CH}_{2}$ & $\mathrm{CH}_{2}$ & $\mathrm{CO}$ & $\mathrm{CH}_{2}$ \\
\hline \multicolumn{2}{|c|}{$\begin{array}{l}\text { Coordination } \\
\text { propyl-linkage } \\
\text { with respect to } \\
\mathrm{C}=\mathrm{O} \text {-group }\end{array}$} & - & - & cis & trans & cis & trans & cis & trans & - & - \\
\hline \multirow{4}{*}{ 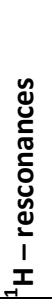 } & TPMA $^{1,2}$ & - & - & 0.89 & 0.92 & 1.57 & 1.61 & 3.29 & 3.32 & - & 3.46 \\
\hline & TTPMA $^{1,3}$ & 1.14 & 3.74 & \multicolumn{2}{|l|}{$0.51^{4,5}$} & $1.57^{4}$ & $1.59^{4}$ & $3.22^{4}$ & $3.25^{4}$ & - & 3.38 \\
\hline & BTESPA $^{1,3}$ & 1.17 & 3.77 & \multicolumn{2}{|l|}{$0.58^{6}$} & \multicolumn{2}{|l|}{$1.55^{6}$} & \multicolumn{2}{|l|}{$2.55^{6}$} & - & - \\
\hline & $\begin{array}{l}\text { Malonyl } \\
\text { chloride }^{3}\end{array}$ & - & - & - & - & - & - & - & - & - & 4.29 \\
\hline \multirow{4}{*}{ 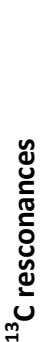 } & TPMA $^{1,2}$ & - & - & 11.23 & 11.36 & 20.81 & 22.16 & 47.7 & 50.1 & 167 & 40.8 \\
\hline & TTPMA $^{1,3}$ & 18.3 & 58.4 & \multicolumn{2}{|l|}{7.57} & 20.8 & 22.3 & 48.6 & 50.9 & 167 & 41.0 \\
\hline & BTESPA $^{1,3}$ & 18.3 & 58.3 & \multicolumn{2}{|l|}{7.96} & \multicolumn{2}{|l|}{23.4} & \multicolumn{2}{|l|}{52.7} & - & - \\
\hline & $\begin{array}{l}\text { Malonyl } \\
\text { chloride }^{3}\end{array}$ & - & - & - & - & - & - & - & - & 164 & 61.3 \\
\hline \multicolumn{12}{|c|}{$\begin{array}{l}1 \text { BTESPA refers to bis-(3-(triethoxysilyl)-propyl)-amine, TTPMA refers to } N, N, N^{\prime}, N^{\prime} \text {-tetrakis-(3- } \\
\text { (triethoxysilyl)-propyl)-malonamide and TPMA refers to } N, N, N^{\prime}, N^{\prime} \text {-tetra- } n \text {-propyl-malonamide. }{ }^{2} \text { Data } \\
\text { from Spectral Data Base System (SDBS) of organic compunds. }{ }^{3} \text { Experimental data from this work. }{ }^{4} \text { Data } \\
\text { assigned by using HSQC-spectrum. }{ }^{5} \text { No difference was observed between B1a and B1b resonances in the } \\
\text { case of TTPMA. Most likely the CO group had a negligible influence on the electron repelling silicon atom } \\
\text { next to this } \mathrm{CH}_{2} \text {-group, which is absent in the TPMA-molecule. }{ }^{6} \text { In the case of BTESPA both } \mathrm{CH}_{2} \text {-goups } \\
\text { are chemically identical, since due to the absence of a double bond the bonds can freely torse and no } \\
\text { distiction between cis-and trans-coordinated atoms can be detected. }\end{array}$} \\
\hline
\end{tabular}




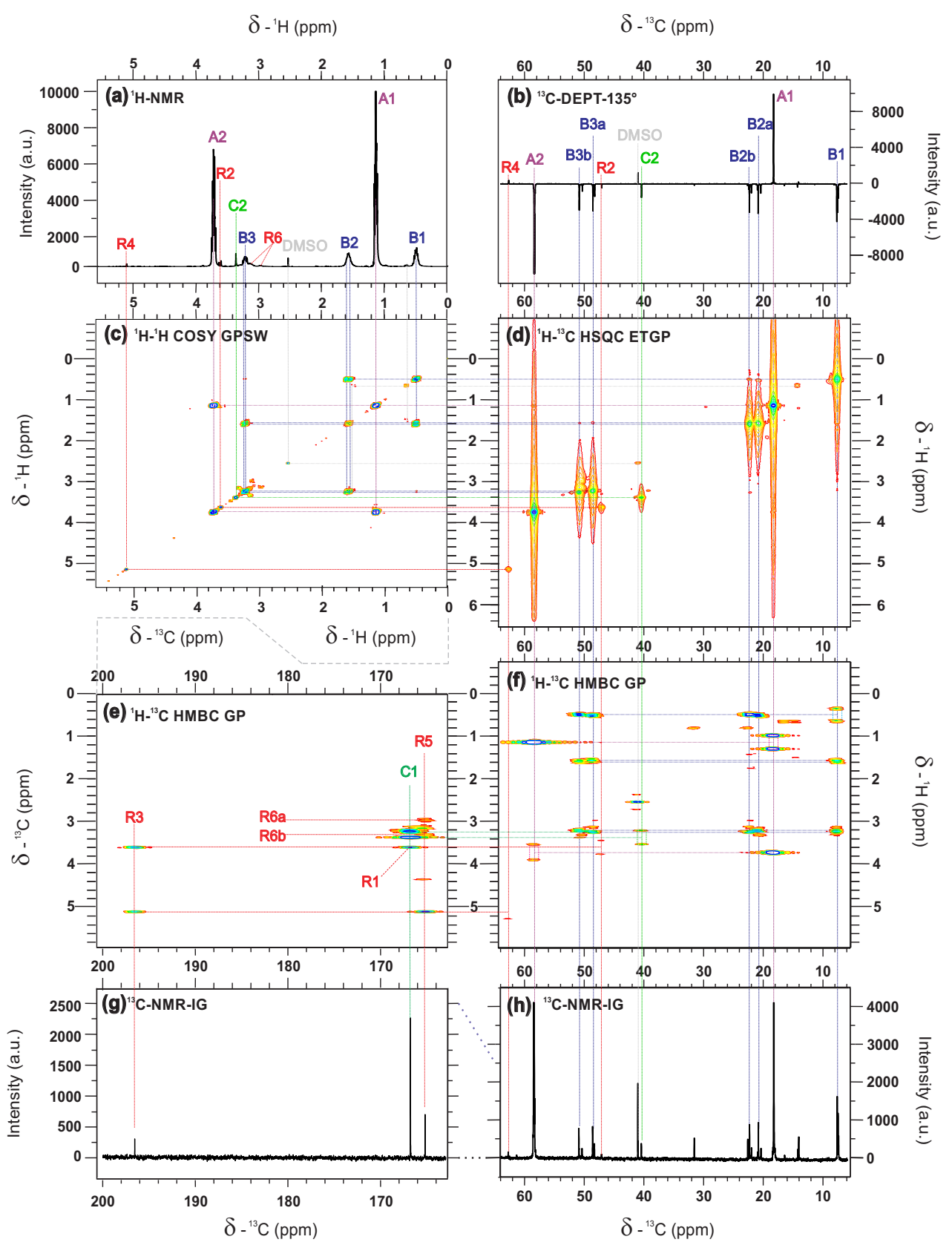


Figure 7.1 (left page) 1H- and 13C-NMR spectra of synthesized TTPMA dissolved in deuterated chloroform. (a) 1H-NMR spectrum, (b) 13C-DEPT-135 spectrum, (c) 1H-1H COSY spectrum, (d) 1H-13C HSQC spectrum, (e-f) 1H-13C HMBC spectrum, with $(e)$ a region of carbonylic carbon atoms $(C=0)$ and $(f)$ a region of methyl, methylene and methine carbon atoms (CHX), and (g-h) inverse gated 13C-NMR spectrum, with $(\mathrm{g})$ a region of carbonylic carbon atoms $(\mathrm{C}=\mathrm{O})$ and $(\mathrm{h})$ a region of methyl, methylene and methine carbon atoms (CHX). Correlations between the resonances are clarified with dotted lines that connect them. The resonances of TTPMA were divided into three categories: (A) CHX-resonances of the ethoxide groups that were designated as $\mathrm{A} 1$ and $\mathrm{A} 2$, and connected by purple dotted lines; (B) CHX-resonances of the bridging propylene linkages that were designated as B1-B3(a,b) and connected by blue dotted lines; and (C) C=O and CH2-resonances of the chelating malonamide segment designated as $\mathrm{C} 1$ and $\mathrm{C} 2$ and connected by green dotted lines. In addition, the resonances designated as R1-R6 belong to a byproduct that was formed during the reaction and connected by the red dotted lines. The chemical groups that belong to the given designations are shown in Figure 7.2.
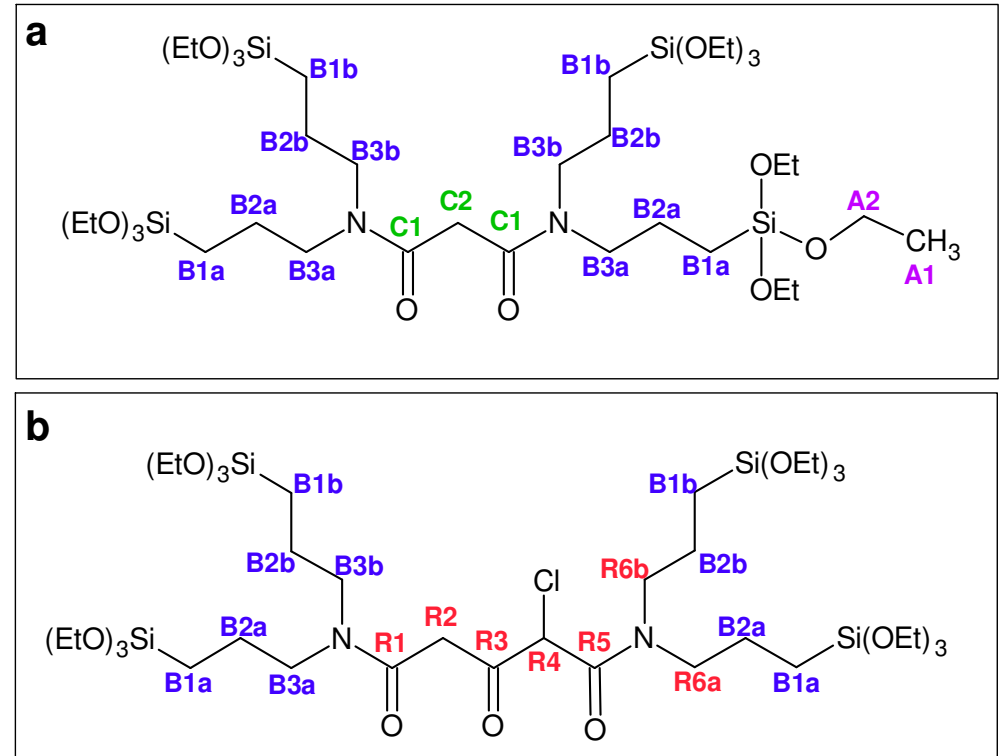

Figure 7.2. Clarification of proton and ${ }^{13} \mathrm{C}-\mathrm{NMR}$ assignments with respect to their positions within the chemical structure. (a) TTPMA (N,N,N',N'-tetrakis-(3(triethoxysilyl)-propyl)-malonamide); (b) a byproduct, most likely (2-chloro-3-oxo$N, N, N^{\prime}, N^{\prime}$-tetrakis-(3-(triethoxysilyl)-propyl)-pentanediamide). 
Table 7.2. List of remnant ${ }^{1} \mathrm{H}$ - and ${ }^{13} \mathrm{C}$-NMR resonances assigned to the byproduct 2-chloro-3-oxo-N,N,N',N'-tetrakis-(3-(triethoxysilyl)-propyl)-pentanediamide.

\begin{tabular}{|l|l|l|l|l|l|l|l|}
\hline Assignment & R1 & R2 & R3 & R4 & R5 & R6a & R6b \\
\hline Group & $\mathrm{C}=\mathrm{O}$ & $\mathrm{CH}_{2}$ & $\mathrm{C}=\mathrm{O}$ & $\mathrm{CHCl}$ & $\mathrm{C}=\mathrm{O}$ & $\mathrm{CH}_{2}$ & $\mathrm{CH}_{2}$ \\
\hline$\delta{ }^{1} \mathrm{H}(\mathrm{ppm})$ & - & 3.62 & - & 5.13 & - & 3.32 & 2.97 \\
\hline$\delta{ }^{13} \mathrm{C}(\mathrm{ppm})$ & 167 & 47.1 & 197 & 62.7 & 165 & 48.4 & 50.5 \\
\hline
\end{tabular}

\subsubsection{Ce-TTPMA complexation}

The FTIR-spectrum of TTPMA (Figure 7.3a) in the absence of cerium tetraisopropoxide isopropanol adduct (CeTiP) revealed a vibration at $\tilde{v}=1638$ $\mathrm{cm}^{-1}$ that was associated with the $\mathrm{C}=0$ vibration of a malonamide $[39,53$, 57]. As CeTiP was added (Figure 7.3c-f), new vibrations arose at $\tilde{v}=1589$ and $1513 \mathrm{~cm}^{-1}$. The shoulder at $1589 \mathrm{~cm}^{-1}$ was caused by a reduced force constant when the free $\mathrm{C}=\mathrm{O}$ moiety was engaged by a metal cation, and the vibration at $\tilde{v}=1513 \mathrm{~cm}^{-1}$ implies the presence of enolic entities $[39,57,61$, 62]. Upon further addition of CeTiP additional vibrations were found at $\tilde{v}=$ 1595 and $1554 \mathrm{~cm}^{-1}$, respectively, which should be associated with a different type of enolic $v \mathrm{C}=\mathrm{O}$ vibration.

Probably, in analogy with the complexation of $\beta$-diketones with zirconium and hafnium isopropoxide, the latter vibrations may be associated with the formation of a mixed malonamide/isopropoxide dimer, while the former could be designated to monomeric species [62]. At the highest ratio (Figure 7.3f) the free amide $\mathrm{C}=\mathrm{O}$ peak $\left(\tilde{v}=1638 \mathrm{~cm}^{-1}\right)$ was not completely diminished, indicating the presence of some unbound malonamide ligand. Meanwhile, vibrations arose at $\tilde{v}=1327,834$ and $816 \mathrm{~cm}^{-1}$ that were associated with unbound CeTiP [63]. Besides the interaction between cerium and the malonamide group, additional vibrations also arose at $\tilde{v}=$ 1039, 903 and $883 \mathrm{~cm}^{-1}$. This indicated the presence of additional electrostatic $\mu$-bridged bonds of oxygen atoms between the ethoxide groups of TTPMA with the cerium cation and/or the isopropoxide groups of CeTiP and a silicon cation. Hence, this gave rise to new vibrations with reduced force constants as compared to the free, unbound molecules.

The exact structure of this complex seems to be rather complex. However, all electrostatic interactions strongly enhance the dispersion of cerium, 
making TTPMA a suitable candidate to disperse cerium within a hybrid silica matrix.
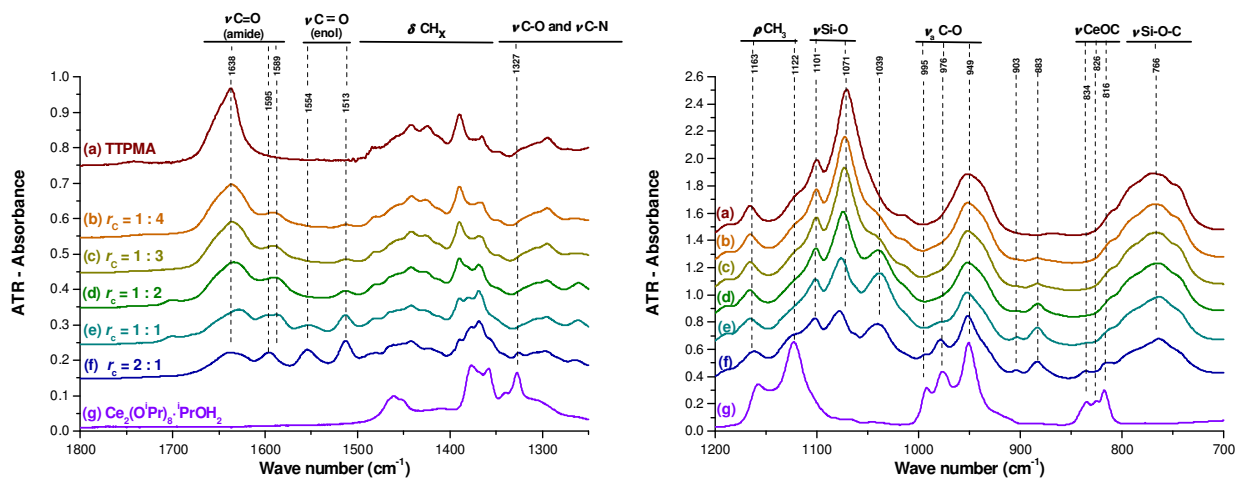

Figure 7.3. FTIR-ATR absorbance spectra of Ce/TTPMA mixtures in the absence of a solvent. Left: $1800-1250 \mathrm{~cm}^{-1}$ and right $1200-700 \mathrm{~cm}^{-1}$. The intensity of the vibrations are weaker in the $1800-1250 \mathrm{~cm}^{-1}$ region and its scale is therefore enhanced relative to the $1200-700 \mathrm{~cm}^{-1}$ region. The lower intensity in the 1800 $1250 \mathrm{~cm}^{-1}$ region was partly due to substantial absorption by the diamond ATR and the strong contributions from the siloxane bonds in the $1200-700 \mathrm{~cm}^{-1}$ region. ATR-FTIR spectra of (a) as-synthesized TTPMA; (b-f) mixture of TTPMA and cerium(IV) isopropoxide isopropanol adduct in different molar ratios: $r_{\mathrm{c}}=$ [Ce]/[TTPMA], where $r_{\mathrm{c}}$ is $\frac{1}{4}, \frac{1}{3}, \frac{1}{2}, 1$ and 2 for $b-f$, respectively, and (g) cerium (IV) isopropoxide isopropanol adduct as received from the supplier.

\subsubsection{Ce-TTPMA sol preparation}

In the case of the undoped hybrid silica sol from 1,2bis(triethoxysilyl)ethane, an intensity weighted average particle size distribution of $7 \mathrm{~nm}$ was found to be optimal for deposition on these $\gamma$ alumina membrane supports $[1,64]$. Consequently, several additional CeTTPMA sols were made with molar ratios $\left[\mathrm{H}^{+}\right] /[\mathrm{OR}]=1 / 30$ and $1 / 200$, varying temperature $\left(20,40\right.$ and $60^{\circ} \mathrm{C}$ ) and reflux time (30 $\mathrm{min}$ and $60 \mathrm{~min}$ ). Yet, all sols were found to have the same bimodal particle size distribution as shown in Figure 7.4. Among these sols the Ce-TTPMA sol with $\left[\mathrm{H}^{+}\right] /[\mathrm{OR}]=$ $1 / 30, \mathrm{~T}=60^{\circ} \mathrm{C}$, and $\mathrm{t}=60 \mathrm{~min}$, with intensity weighed hydrodynamic diameters of 2.8 and $34 \mathrm{~nm}$ and an overall average particle size of $13 \mathrm{~nm}$ (Figure 7.4) was considered to match the optimal particle size distribution of $\sim 7 \mathrm{~nm}$ most closely. 


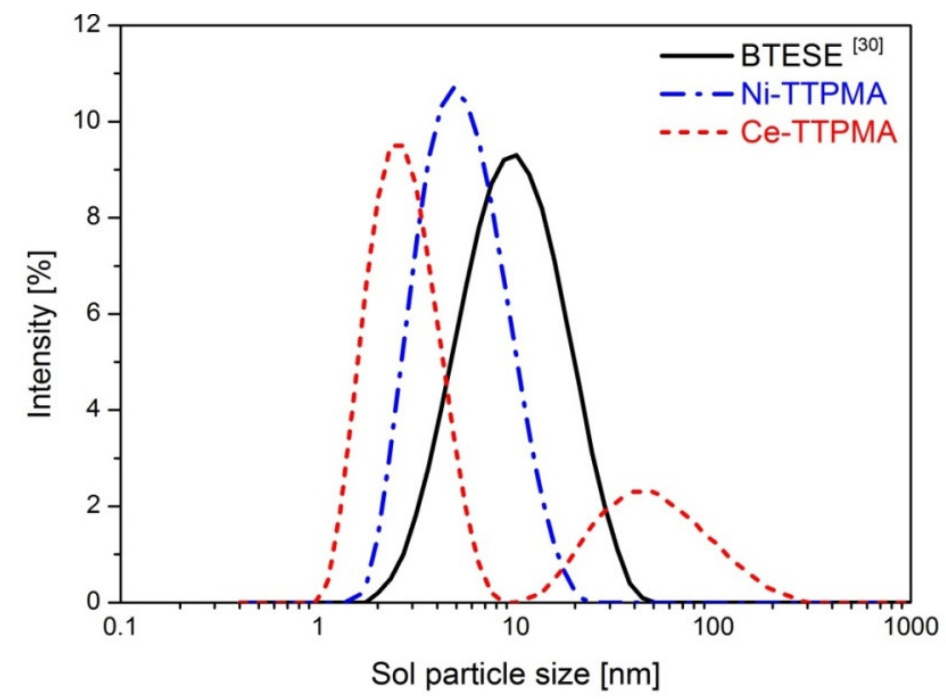

Figure 7.4. Sol particle size distributions of Ce-TTPMA and Ni-TTPMA sols obtained by dynamic light scattering. Results are compared with a previously described recipe of undoped hybrid 1,2-bis-(triethoxysilyl)ethane (BTESE) [1].

\subsubsection{Dispersion of cerium within the condensed TTPMA matrix}

The FTIR-spectrum of the as-prepared sol of Ce-TTPMA with $r_{\mathrm{C}}=\frac{1}{2}$ revealed a broad peak in the range between $1680-1580 \mathrm{~cm}^{-1}$ (Figure 7.5a). It is a convoluted signal that consists of water deformations $\left(\delta \mathrm{H}_{2} \mathrm{O}\right)$ and carbonyl vibrations $(v \mathrm{C}=\mathrm{O})$. Due to the overlap with $\delta \mathrm{H}_{2} \mathrm{O}$ and the fact that the diluted sol has a weak $v \mathrm{C}=\mathrm{O}$ contribution, it is difficult to discriminate between the different entities. When the sol was coated on a silicon wafer and dried at $T=60^{\circ} \mathrm{C}$, the water contribution was diminished and a more pronounced $v \mathrm{C}=0$ was observed with a maximum at $\tilde{v}=1604 \mathrm{~cm}^{-1}$ (Figure 7.5b). This vibration clearly shifted with respect to the free malonamide $v \mathrm{C}=\mathrm{O}$ vibration at $\tilde{v}=1628 \mathrm{~cm}^{-1}$ (Figure $7.5 \mathrm{~d}$ ), although no additional peak arose near $\tilde{v}=1628 \mathrm{~cm}^{-1}$ as was observed for the mixture of TTPMA and CeTiP before hydrolysis and condensation (Figure 7.3d(left)). After calcining the sample at $T=250^{\circ} \mathrm{C}$ for $3 \mathrm{~h}$ a new vibration appeared at $\tilde{v}=1637 \mathrm{~cm}^{-1}$, which is very close to the maximum of $v \mathrm{C}=\mathrm{O}$ of liquid TTPMA (Figure 7.3a). Meanwhile, the absorbance vibration near $\tilde{v}=1604 \mathrm{~cm}^{-1}$ clearly decreased, 
which seems to correspond with a reduction of the number of malonamide ligands that are coordinated to $\mathrm{Ce}^{4+}$.

In conclusion, these FTIR measurements clearly reveal malonamide ligands being coordinated to $\mathrm{Ce}^{4+}$. The nature of this complex (monomeric, dimeric, etc.) depends on the complexation ratio. However, the exact structure could not be resolved solely by FTIR. Cerium remains coordinated to the TTPMA ligand during sol-gel processing and drying. However, during calcination at $\mathrm{T}=250^{\circ} \mathrm{C}$ a substantial amount of malonamide ligands became detached from cerium, which may subsequently have phase separated in the form of ceria.

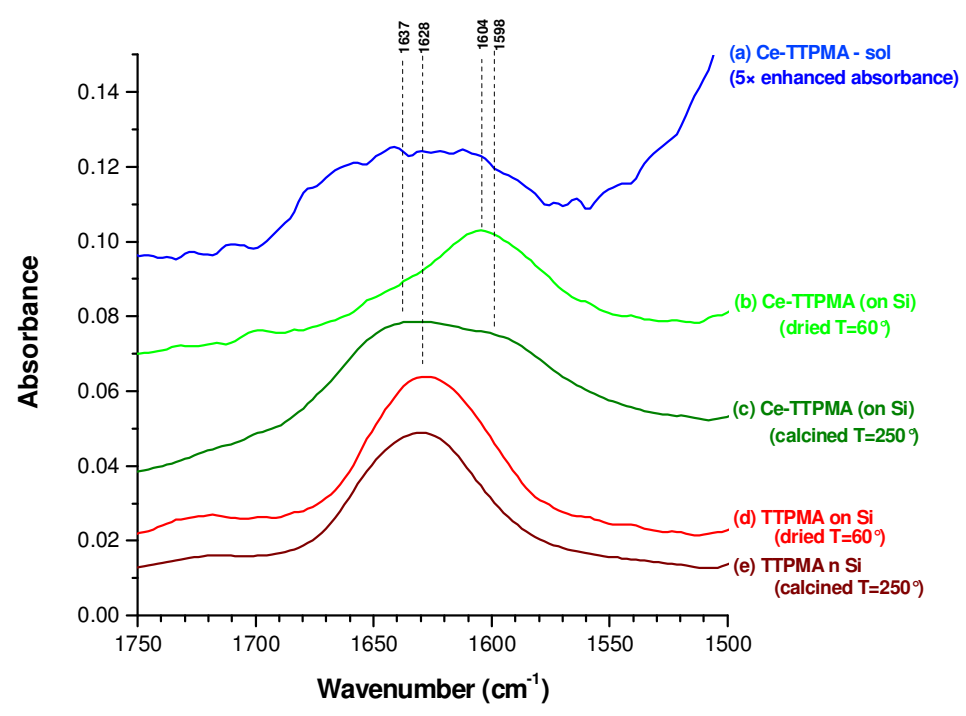

Figure 7.5 FTIR-ATR absorbance spectra (carbonyl-region). (a) as prepared CeTTPMA sol; (b-c) spin-coated Ce-TTPMA sol on a plasma oxidized silicon wafer, where (b) was dried at $T=60^{\circ} \mathrm{C}$ and (c) calcined at $T=250^{\circ} \mathrm{C}$; (d-e) spincoated TTPMA-sol without ceria, where (d) was dried at $T=60^{\circ} \mathrm{C}$ and (e) calcined at $T=250^{\circ} \mathrm{C}$.

TEM measurements were performed on calcined samples of dipcoated holey TEM grids to gain insight into the dispersion of ceria within the hybrid silica matrix. These data revealed the phase separation of small nanosized (2-5 nm) crystallites within an amorphous matrix (Figure 7.6a). The crystallites were found to have lattice spacings of $d^{\sim 3.1} \AA$, as indicated by 
the encircled particle (Figure 7.6a) and its fast fourier transform image (Figure 7.6b). This value is consistent with $\mathrm{CeO}_{2}$ (111) planes [65].

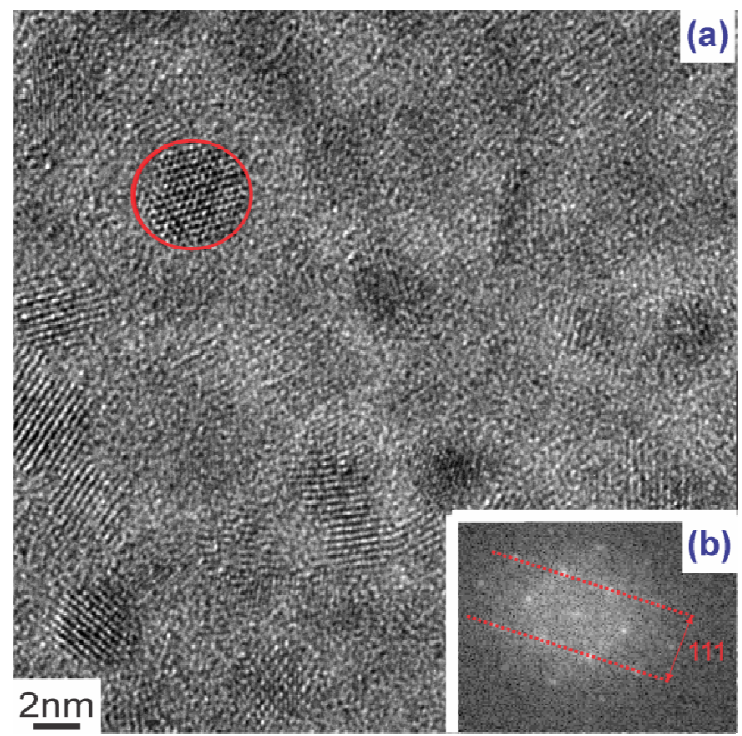

Figure 7.6 (a) HR-TEM images of a holey copper grid (with a carbon film) dipcoated with Ce-TTPMA sol and calcined at $T=250{ }^{\circ} \mathrm{C}$. (b) fourier transform of the encircled region in section (b) of this figure.

\subsubsection{Membrane characteristics of the Ce-TTPMA coated layer}

After dipcoating the diluted Ce-TTPMA sol and calcination at $T=250^{\circ} \mathrm{C}$ a thin selective layer of approx. $35 \mathrm{~nm}$ was formed on top of the mesoporous $\gamma$ $\mathrm{Al}_{2} \mathrm{O}_{3}$ layer, as shown by the secondary electron HR-SEM-image (Figure 7.7a). The smooth Ce-TTPMA coating seems to penetrate partly into the mesoporous $\gamma-\mathrm{Al}_{2} \mathrm{O}_{3}$ layer. This observation is supported by an image that was made by using the energy selective backscatter (ESB) detector (Figure 7.7b), which is particularly sensitive to electron rich elements such as cerium. The data revealed that the cerium containing layer was approx. 58 $\mathrm{nm}$ thick, $23 \mathrm{~nm}$ thicker than determined by the secondary electron detector (SE2) that is more sensitive to surface morphology. Probably, the Ce-TTPMA sol particles were smaller than the pore size of the $\gamma-\mathrm{Al}_{2} \mathrm{O}_{3}$ layer, resulting in partial penetration into the mesoporous $\gamma-\mathrm{Al}_{2} \mathrm{O}_{3}$ layer. 


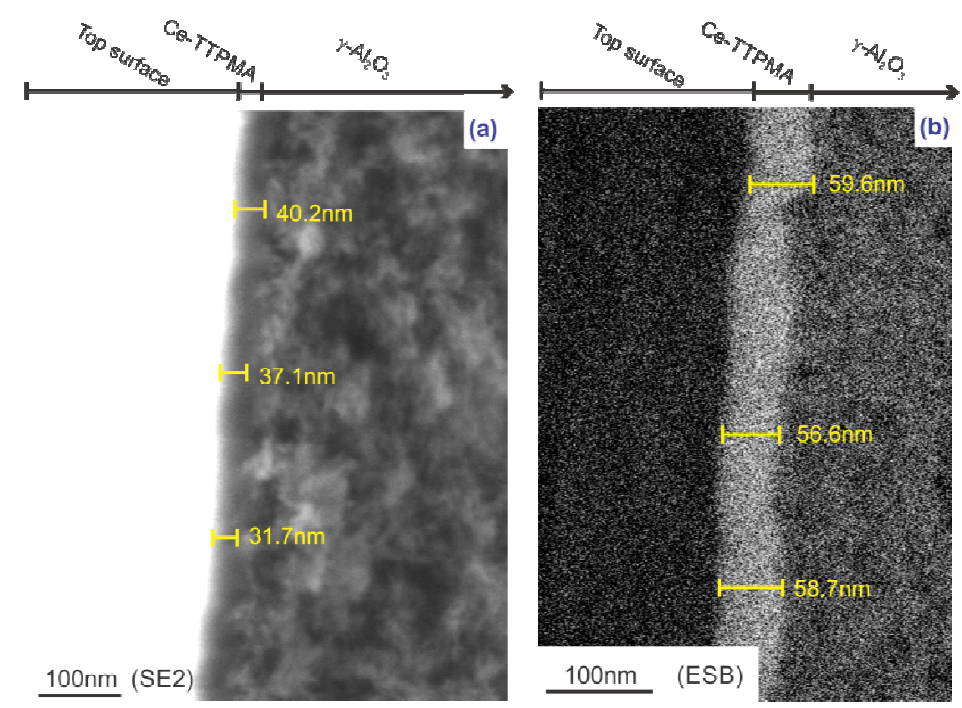

Figure 7.7 HR-SEM images of a Ce-TTPMA top layer on a meso/macroporous $\gamma$ $\mathrm{Al}_{2} \mathrm{O}_{3} / \alpha-\mathrm{Al}_{2} \mathrm{O}_{3}$ support. (a) secondary electron detector (SE2) image; (b) energy selective backscatter detector (ESB).

Ce-doped TTPMA membrane with an $\mathrm{H}_{2} / \mathrm{N}_{2}$ permselectivity of 20 showed a slightly better performance than a BTESE (1,2-bis-(triethoxysilyl)-ethane) based membrane with $\mathrm{H}_{2} / \mathrm{N}_{2}=14$ as reported elsewhere [1], even though the layer thickness was much smaller $(50-60 \mathrm{~nm})$ than that of the BTESE membrane ( $260 \mathrm{~nm}$ ). Despite the reduced film thickness and bimodal sol size distribution, no permeation of $\mathrm{SF}_{6}$ was observed. This indicates a pore size of less than $0.55 \mathrm{~nm}$ and the absence of large defects. Moreover, only a minimal variation of less than $5 \%$ was observed in the hydrogen permeance between start and at end of the gas permeation analyses. This indicates that Ce-TTPMA derived membranes have a sustainable pore microstructure for gas separation.

The $\mathrm{H}_{2} / \mathrm{CO}_{2}$ permselectivity of 6 was slightly larger than its corresponding Knudsen diffusion value of 4.7. Since the resistance towards $\mathrm{N}_{2}$ increased to the same extent as $\mathrm{CO}_{2}$, the increased performance was not associated with affinity, but mainly with narrowing of the pore size distribution. Similar helium and hydrogen fluxes were obtained with a substantially thinner selective layer. Thus, the selective layer was much more resistive than the BTESE membrane. This may have several causes: (1) narrower pores, (2) $\mathrm{CeO}_{2}$ particles that effectively block pores, and/or (3) lower porosity. 


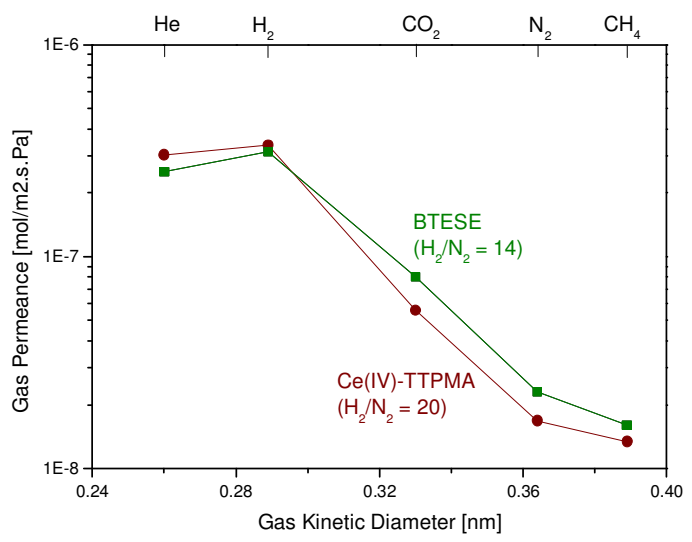

Figure 7.8. Single gas permeance of a Ce-TTPMA membrane and a hybrid BTESE membrane [1] on a macroporous $\alpha-\mathrm{Al}_{2} \mathrm{O}_{3}$ supported mesoporous $\gamma-\mathrm{Al}_{2} \mathrm{O}_{3}$ layer.

\subsubsection{Nickel(II)-TTPMA-complexation}

In the region between $\tilde{v}=1730$ and $1620 \mathrm{~cm}^{-1}$ a broad peak was observed for a $0.4 \mathrm{M} \mathrm{Ni}\left(\mathrm{NO}_{3}\right)_{2} \cdot 6 \mathrm{H}_{2} \mathrm{O}$ solution in ethanol (Figure 7.9a (left)) which was caused by $\mathrm{H}_{2} \mathrm{O}$ deformations. The maximum was shifted to higher frequencies compared to free water due to electrostatic interactions with nickel [66]. These deformations overlap with $\mathrm{C}=\mathrm{O}$ vibrations when studying the complexation of nickel with TTPMA. However, they were substantially broader than the $\mathrm{C}=\mathrm{O}$ vibrations and seemed to be distinguishable.

When $\mathrm{Ni}\left(\mathrm{NO}_{3}\right)_{2} \cdot 6 \mathrm{H}_{2} \mathrm{O}$ was mixed with TTPMA new vibrations arose at $\tilde{v}=$ 1612 and $1590 \mathrm{~cm}^{-1}$, which led to reduced force constants when $(\mathrm{C}=\mathrm{O})$ vibrations were electrostatically coordinated to $\mathrm{Ni}^{2+}$ (Figure 7.9b-e (left)), similar to the CeTiP precursor described above. Upon increasing the nickel content the vibration at $\tilde{v}=1590 \mathrm{~cm}^{-1}$ increased, while the vibration at $\tilde{v}=$ $1612 \mathrm{~cm}^{-1}$ diminished. Both vibrations can be associated with different $\mathrm{Ni}-$ malonamide entities, for instance with different coordination number of malonamide ligands on $\mathrm{Ni}^{2+}$. For the sake of comparison $\mathrm{Ni}\left(\mathrm{NO}_{3}\right)_{2} \cdot 6 \mathrm{H}_{2} \mathrm{O}$ was also mixed with tetrapropylmalonamide (Figure 7.9f-g (left)). Similar to the Ni-TTPMA complex, the vibration $\tilde{v}=1612 \mathrm{~cm}^{-1}$ was predominant at a low complexation ratio $\left(r_{C}=\frac{1}{4}\right)$, while the vibration $\tilde{v}=1590 \mathrm{~cm}^{-1}$ was predominant at higher complexation ratio $\left(r_{\mathrm{C}}=1\right)$. Moreover, for the $\mathrm{Ni}$ TTPMA samples with $r_{\mathrm{C}}=1$ or 2 a vibration was clearly observed at $1527 \mathrm{~cm}^{-}$ 1 which is associated with chelated bidentate nitrate ions [42, 67], 
indicating that some nitrate ions remain coordinated to nickel upon complexation.

During complexation the water molecules coordinated to nickel were replaced by malonamide ligands. The released water partly hydrolyzed the ethoxide groups of TTPMA, as suggested by the decreasing intensity of $v \operatorname{SiOC}\left(\tilde{v}=958 \mathrm{~cm}^{-1}\right)$ with increasing $r_{\mathrm{C}}$ (Figure 7.9b-f (right)) [68, 69]. This was accompanied by the increasing intensity of $v \mathrm{SiOH}\left(\tilde{v}=920 \mathrm{~cm}^{-1}\right)$. Also, the $\mathrm{H}_{2} \mathrm{O}$ deformations ( $\tilde{v}=1730$ and $1620 \mathrm{~cm}^{-1}$ ) almost diminished upon aging, and significant $\mathrm{H}_{2} \mathrm{O}$ deformation was only observed at the highest complexation ratio (Figure $7.9 \mathrm{f}$ (left)). Furthermore, a small increase of $v$ SiOSi $\left(\tilde{v}=1010 \mathrm{~cm}^{-1}\right)$ indicated that some $\mathrm{SiOH}$ entities condensed to form Si-O-Si bridges $[70,71]$ within $2 \mathrm{~h}$ of aging.
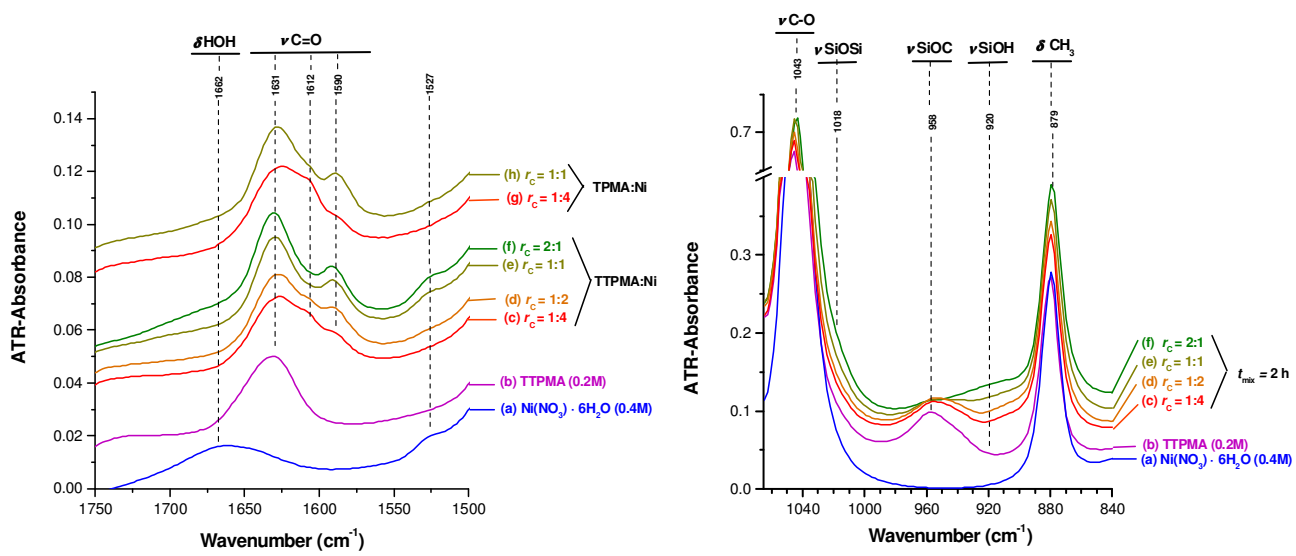

Figure 7.9. FTIR-ATR absorbance spectra of $\mathrm{Ni}\left(\mathrm{NO}_{3}\right)_{2} /$ TTPMA solutions in ethanol. Left: $1750-1500 \mathrm{~cm}^{-1}$ and right: $1065-840 \mathrm{~cm}^{-1}$. The intensity of the vibrations are weaker in the former region and its scale is therefore enhanced relative to the right figure. The lower intensity in the $1750-1500 \mathrm{~cm}^{-1}$ region was partly due to substantial absorption by the diamond ATR and the strong contributions from the siloxane bonds in the $1065-840 \mathrm{~cm}^{-1}$ region. ATR-FTIR spectra of (a) $0.4 \mathrm{~mol} / \mathrm{L}$ $\mathrm{Ni}\left(\mathrm{NO}_{3}\right)_{2} \cdot 6 \mathrm{H}_{2} \mathrm{O}$ solution in ethanol; (b) $0.2 \mathrm{~mol} / \mathrm{L}$ TTPMA solution in ethanol; (c-f) $0.2 \mathrm{~mol} / \mathrm{L}$ TTPMA-Ni($\left(\mathrm{NO}_{3}\right)_{2} \cdot 6 \mathrm{H}_{2} \mathrm{O}$ solutions in ethanol with $r_{\mathrm{C}}=[\mathrm{Ni}] /[\mathrm{TTPMA}]=\frac{1}{4}$, $\frac{1}{2}, 1$ and 2 for $c-f$, respectively, after $2 \mathrm{~h}$ aging at room temperature; $(\mathrm{g}, \mathrm{h}) 0.2$ $\mathrm{mol} / \mathrm{L} \mathrm{TTPMA-Ni}\left(\mathrm{NO}_{3}\right)_{2} \cdot 6 \mathrm{H}_{2} \mathrm{O}$ solutions in ethanol with $r_{\mathrm{C}}=[\mathrm{Ni}] /[\mathrm{TPMA}]=\frac{1}{4}$ and 1 for $f$ and $g$, respectively, after $2 \mathrm{~h}$ aging at room temperature. 
The extent of complexation was also found to be time-dependent, as indicated by FTIR experiments for different aging times at fixed ratio $r_{\mathrm{C}}=1$, see Figure 7.10 (left). Immediately after aging, two carbonyl vibrations were observed: one at $\tilde{v}=1631 \mathrm{~cm}^{-1}$ that is associated with the free malonamide ligand and another vibration at $\tilde{v}=1590 \mathrm{~cm}^{-1}$. With increasing aging time at room temperature a vibration arose at $\tilde{v}=1612 \mathrm{~cm}^{-1}$, which became the predominant carbonyl vibration after $8 \mathrm{~h}$ of ageing. Apparently, this complex was slowly changing into a different conformation. Then, for the sake of comparison the same time-dependent experiment was performed with TPMA. Initially, at $t=0$ the $\mathrm{Ni}$-TPMA spectrum had the same $\mathrm{C}=\mathrm{O}$ vibrations with similar intensities as $\mathrm{Ni}$-TTPMA. However, unlike $\mathrm{C}=\mathrm{O}$ vibrations of Ni-TTPMA, the $\mathrm{C}=\mathrm{O}$ vibrations of $\mathrm{Ni}$-TPMA remained unchanged for $24 \mathrm{~h}$. Thus, in the initial stage Ni-TTPMA and Ni-TPMA have similar conformations. However, the additional triethoxysilane groups cause the Ni-TTPMA to gradually rearrange its conformation upon ageing the Ni-TTPMA-mixture, while for Ni-TPMA the original conformation remained intact.

In uranium complexes of tetra-substituted malonamides an additional $\mathrm{C}=\mathrm{O}$ vibration $\left(1604 \mathrm{~cm}^{-1}\right)$ in between the $\mathrm{C}=\mathrm{O}$ vibration of free malonamide $\left(1623 \mathrm{~cm}^{-1}\right)$ and single bonded malonamide $\left(1592 \mathrm{~cm}^{-1}\right)$ was associated with a double bonded malonamide [42]. The authors supported their claim by the fact that upon reducing $r_{\mathrm{C}}$ from 1 to $\frac{1}{2}$, the vibration at $1604 \mathrm{~cm}^{-1}$ became predominant while the bidentate chelating nitrate vibration at $1524 \mathrm{~cm}^{-1}$ diminished, similar to our case. This suggestion is plausible since when a nitrate ligand is replaced by a more nucleophilic malonamide, the acidity of nickel is reduced. Consequently, nickel will have a smaller effect on the force constant of the $\mathrm{C}=\mathrm{O}$ vibration and this vibration will shift towards the frequency of a free ligand. A similar shift was observed for $\mathrm{Ni}(\mathrm{acac})_{2} \mathrm{~L}$ complexes when increasing the nucleophilic character of the bidentate ligand $L$ [72]. Thus, the gradual change Ni-TTPMA spectrum could be associated to a redistribution of a single malonamide bond to $\mathrm{Ni}^{2+}$ towards a complex of multiple malonamide ligands coordinated to $\mathrm{Ni}^{2+}$. This also means that due to rearrangement of this complex at $r_{C}=1$, at least half of the $\mathrm{Ni}^{2+}$ ions were not coordinated to malonamide. Remarkably, this rearrangement did not occur for Ni-TPMA and the presence of the triethoxysilyl groups may be responsible for this rearrangements process.

As the aging time increased, the extent of hydrolysis and condensation also increased. After $24 \mathrm{~h}$ the SiOC vibration diminished almost completely and 
almost all alkoxide groups were hydrolyzed. The optimal microporosity in acid catalyzed polymeric sols is typically achieved when the extent of hydrolysis and condensation is limited during the mixing stage $[27,73]$.

As evidenced by FTIR, the malonamide ligands were coordinating nickel and enhancing its dispersion within the silsesquioxane matrix. Water that was initially coordinated to nickel was released and caused some hydrolysis and condensation of Si-OEt groups. Moreover, the conformation of the $\mathrm{Ni}$ TTPMA complex changed upon aging mixture. This shift was associated with an increasing number of TTPMA ligands being coordinated to Ni. Due to disproportionation of the Ni-TTPMA complex several Ni-ions would not be coordinated to a malonamide ligand. Both aspects, i.e. hydrolysis/condensation before the addition of acidic catalyst and the release of $\mathrm{Ni}$ being coordinated to TTPMA, do not present an optimal microstructure for the selective membrane layer. Therefore, the aging time should be kept limited. An aging time $t_{\text {mix }}=2 \mathrm{~h}$ was chosen to ensure for some complexation of nickel before preparing the sol.
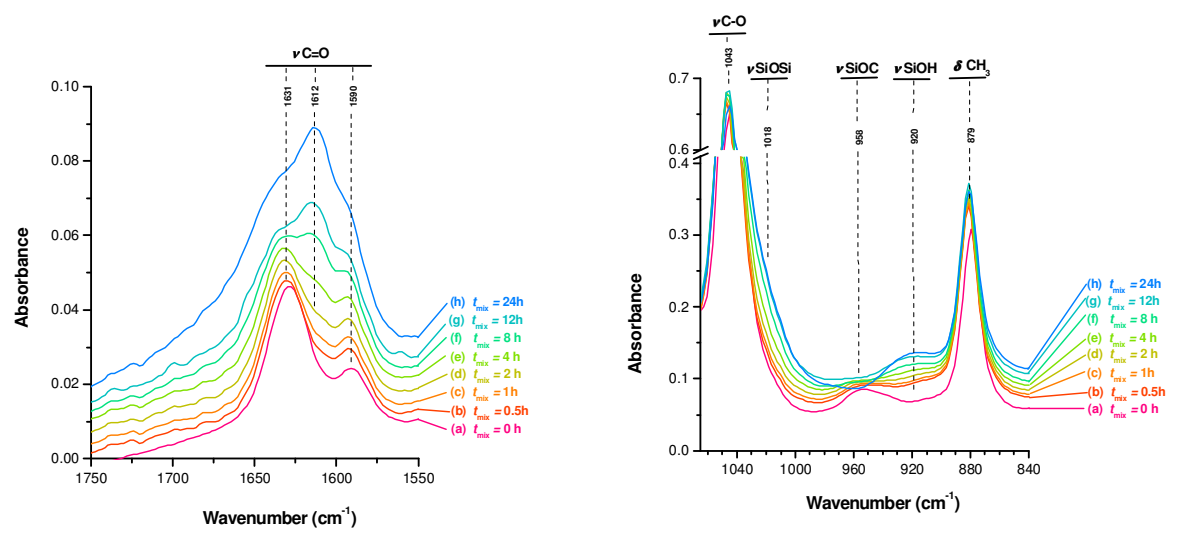

Figure 7.10. FTIR-ATR absorbance spectra of Ni(NO3)2/TTPMA solutions in ethanol, with ageing times after aging of 0, 0.5, 1, 2, 4, 8, 12 and $24 \mathrm{~h}$ for graphs a-h, respectively. Left: $1750-1550 \mathrm{~cm}-1$ and right: $1065-840 \mathrm{~cm}-1$. The intensity of the vibrations are weaker in the $1750-1550 \mathrm{~cm}-1$ region and its scale is therefore enhanced relative to the figure to the right. The lower intensity in the $1750-1550 \mathrm{~cm}-1$ region was partly due to substantial absorption by the diamond ATR, as well as the strong contributions from the siloxane bonds in the 1065 - 840 cm-1 region. 


\subsubsection{Ni-TTPMA-sol preparation}

The sol particle size was easily tuned by the overall concentration [OR], defined here as $[\mathrm{OR}]=[-\mathrm{OEt}]+\left[\mathrm{NO}_{3}^{-}\right]$. Two different acid ratios were employed: $\left[\mathrm{H}^{+}\right] /[\mathrm{OR}]=1 / 30$ and $1 / 15$ (Figure 7.11). The sol particle size clearly increased with [OR] for both acid ratios. The higher acid ratio yielded larger sol particles due to an increased condensation rate. Since TTPMA may contain traces of amines and since the Ce-TTPMA layer was found to be quite resistive to gas permeation, the higher ratio $\left[\mathrm{H}^{+}\right] /[\mathrm{OR}]=1 / 15$ was preferred over the smaller one. This ensured an acid catalyzed hydrolysis and condensation mechanism that typically leads to polymeric sol particles in the case of silicates $[27,74]$. The optimal particle size $(\sim 7 \mathrm{~nm})$ with a monomodal particle size distribution (Figure 7.4) for membrane coating onto an $\gamma-\mathrm{Al}_{2} \mathrm{O}_{3} / \alpha-\mathrm{Al}_{2} \mathrm{O}_{3}$ support $[1,64]$ was found for sols with $\left[\mathrm{H}^{+}\right] /[\mathrm{OR}]=$ $1 / 15$ and $[\mathrm{OR}]=1.2 \mathrm{~mol} / \mathrm{L}$.

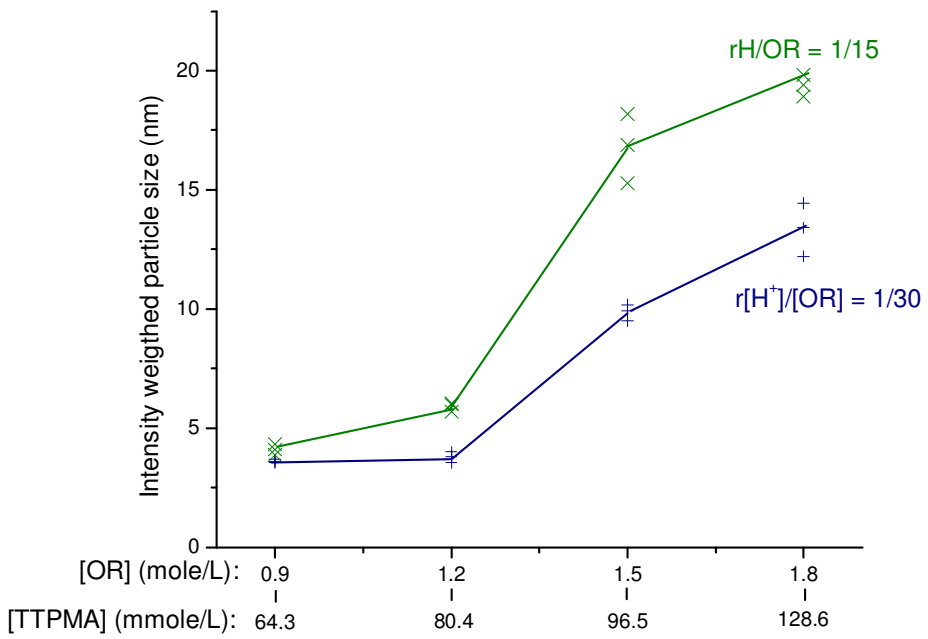

Figure 7.11. Particle size analysis by dynamic light scattering with concentrations $[-O R]$ of $0.9,1.2,1.5$ and 1.8 and $\left[H^{+}\right] /[O R]=1 / 30$ and $1 / 15$. 


\subsubsection{Dispersion of $\mathrm{Ni}^{2+}$ within the condensed TTPMA matrix.}

After sol formation for $1 \mathrm{~h}$ at $T=60^{\circ} \mathrm{C}$ the vibration at $\tilde{v}=1612 \mathrm{~cm}^{-1}$ was clearly enlarged with respect to the vibration at $\tilde{v}=1590 \mathrm{~cm}^{-1}$ (Figure 7.12a). Thus, the system may have reorganized into a mixture of nickel with multiple malonamide ligands and free nickel (nitrate) ions. After coating the sol on a Si-wafer and drying at $T=60^{\circ} \mathrm{C}$, the maximum of the carbonyl vibration was slightly shifted to $\tilde{v}=1604 \mathrm{~cm}^{1}$ (Figure 7.12b). Since this vibration was rather broad, it was not clear whether it should be considered as a different species or as a convolution of the vibrations at $\tilde{v}=1612$ and $1590 \mathrm{~cm}^{-1}$.

However, this vibration was clearly shifted with respect to the coated TTPMA-sol in the absence of nickel (Figure 7.12d), indicating that a substantial amount of nickel remained coordinated to the malonamide ligand. Upon calcination the carbonyl vibration nearly changed (Figure 7.12c) and only a marginal increase of a shoulder at $\tilde{v}=1628 \mathrm{~cm}^{-1}$ was observed. In the calcined Ni-TTPMA sample the decomposition of nitrates clearly occurred as shown by reduction of absorbances in the range between $\tilde{v}=1420,1300 \mathrm{~cm}^{-1}$ and $1040 \mathrm{~cm}^{-1}$ due to reduction of $\mathrm{N}=\mathrm{O}$ and $\mathrm{N}$ $O$ vibrations $[67,75,76]$. The reduction of the $\mathrm{N}-\mathrm{O}$ vibration $\left(1040 \mathrm{~cm}^{-1}\right)$ was less pronounced due to the strong overlap with the increasing asymmetric Si-O-Si vibration ( $\tilde{v}=1007 \mathrm{~cm}^{-1}$ ) [70]. The absence of a sharp vibration at $\tilde{v}$ $=3640 \mathrm{~cm}^{-1}$ indicates the absence of a hydroxyl group attached to nickel that could have formed during decomposition. However, a broad $\mathrm{vOH}(\tilde{\mathrm{v}}=$ $3600-2900 \mathrm{~cm}^{-1}$ ) remained, most likely due to remnant water that is known to remain present in hydrated nickel oxide up to $\mathrm{T}=400^{\circ} \mathrm{C}[76]$.

In summary, FTIR measurements reveal that a substantial amount of $\mathrm{Ni}$ remains coordinated upon calcination at $\mathrm{T}=250^{\circ} \mathrm{C}$. However, not all $\mathrm{Ni}$ ions may be coordinated to malonamide due to the disproportionation of the Ni-TTPMA complex as discussed in section 3.6. Meanwhile, nitrate ions were clearly decomposed, however a substantial amount of water remained present in the microstructure as indicated by the $\mathrm{vOH}$. This could possibly affect the permeability of gases going through the selective membrane layer. 


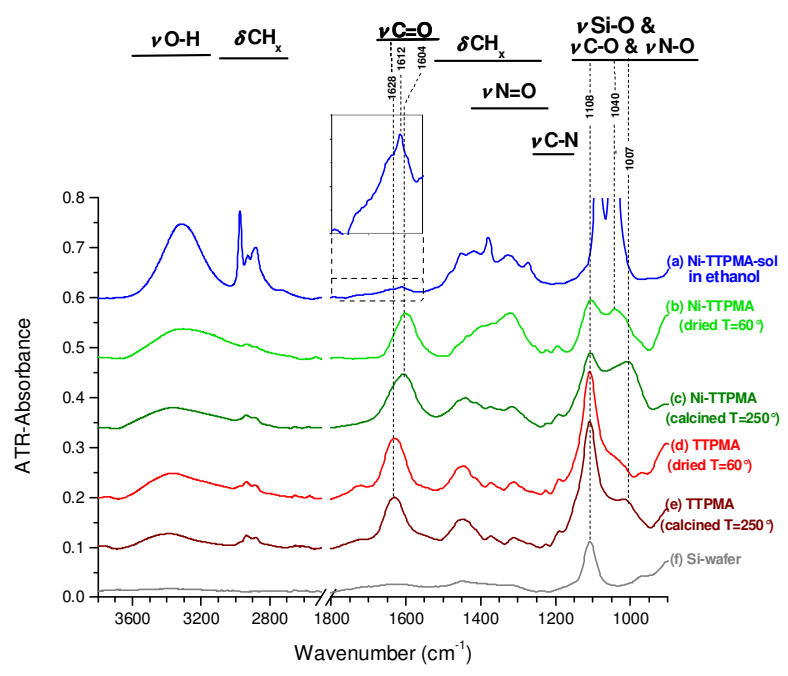

Figure 7.12. FTIR-ATR absorbance spectra. (a) as-prepared Ni-TTPMA sol, with an elongated section of the region with $\mathrm{C}=\mathrm{O}$ vibrations and $\mathrm{H}_{2} \mathrm{O}$ deformations (1800$1550 \mathrm{~cm}^{-1}$ ); (b-c) spincoated Ni-TTPMA sol on a plasma oxidized silicon wafer, where (b) was dried at $T=60^{\circ} \mathrm{C}$ and (c) was calcined at $T=250^{\circ} \mathrm{C}$; (d-e) spincoated TTPMA-sol without cerium ions, where (d) was dried at $T=60^{\circ} \mathrm{C}$ and (e) was calcined at $T=250^{\circ} \mathrm{C}$; (f) clean $\left(\mathrm{O}_{2}\right.$-plasma oxidized) Si-wafer.

The $\mathrm{Ni}-2 \mathrm{p}$ region in the XPS spectrum of the dried and calcined sample were identical within experimental error and therefore only the spectrum of the calcined sample is shown Figure 7.13. The $\mathrm{Ni} 2 \mathrm{p}^{3} / 2$ binding energies at 856.0 $\mathrm{eV}$ and a satellite peak at $861.2 \mathrm{eV}$ clearly revealed the absence of $\mathrm{NiO}$, since $\mathrm{NiO}$ has two main peaks at 854.1 and $856.2 \mathrm{eV}$ and two satellite peaks at 861.2 and $863.8 \mathrm{eV}$ [77].

On the other hand, the energies were consistent with a nickel (II) acetylacetone complex (856.3 eV and a satellite at $861.3 \mathrm{eV}$ ), which will have similar binding energies as nickel coordinated to malonamide ligands, as they both have a $\beta$-diketonate structure [78]. Also an energy difference of $5.2 \mathrm{eV}$ between the main peak and its satellite peak in the current sample implies that the $\mathrm{Ni}$ species has a more ionic character than is found in nickel(II)oxide (7.2 eV) [78]. However, the decomposition of pure $\mathrm{Ni}\left(\mathrm{NO}_{3}\right)_{2} \cdot 6 \mathrm{H}_{2} \mathrm{O}$ in nitrogen atmosphere at $\mathrm{T}=250^{\circ} \mathrm{C}$ has been reported to lead to formation of $\mathrm{Ni}_{2} \mathrm{O}_{3}$ due to partial oxidation caused by the presence of nitrate ions [75]. Different energies have been reported for the main $\mathrm{Ni}$ 2p3/2 peak of $\mathrm{Ni}_{2} \mathrm{O}_{3}$, ranging from 855.6 to $856.9 \mathrm{eV}$ [79-83]. Thus, the 
presence of $\mathrm{Ni}_{2} \mathrm{O}_{3}$ nanoparticles within the TTPMA-coating cannot be excluded.

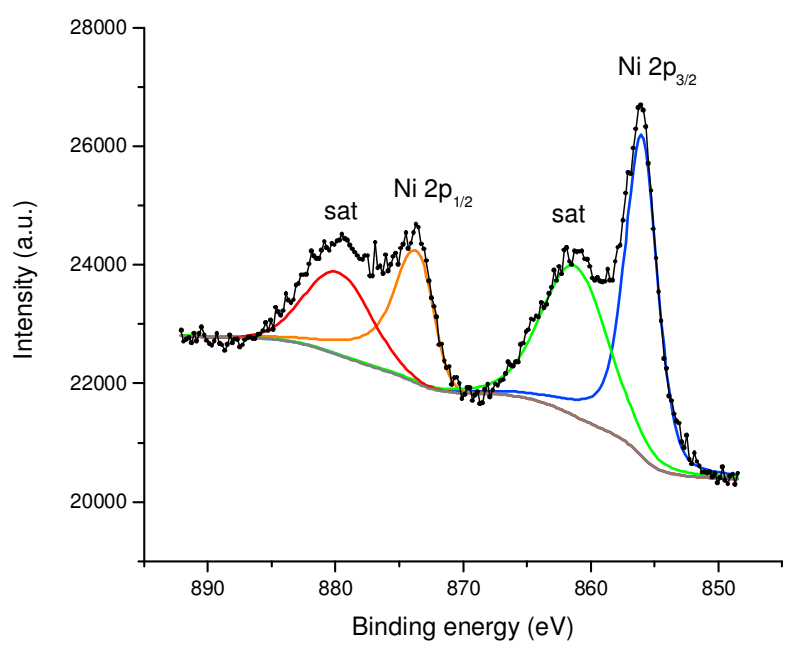

Figure 7.13. XPS-spectrum of Ni-TTPMA-sol after spin-casting on a plasmaoxidized silicon substrate, followed by drying at $T=60^{\circ} \mathrm{C}$ and calcination at $T=250^{\circ} \mathrm{C}$ in $\mathrm{N}_{2}$.
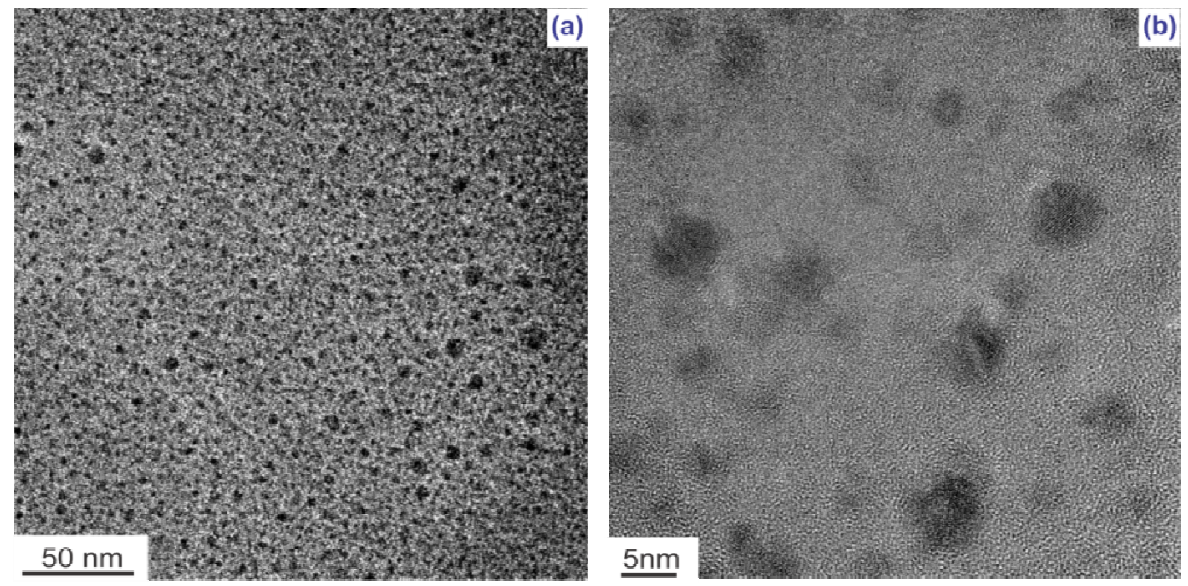

Figure 7.14 HR-TEM images of a holey copper grid dipcoated with Ni-TTPMA after calcination at $T=250{ }^{\circ} \mathrm{C}$ at 2 different length scales. (a) overview image; (b) magnified image showing phase separated particles with sizes between 1 and 15 $\mathrm{nm}$. 
TEM experiments revealed a substantial amount of phase separated electron rich particles. These particles with sizes ranging from 1 to $15 \mathrm{~nm}$ (Figure 7.14) most likely consist of hydrated $\mathrm{Ni}_{2} \mathrm{O}_{3}$, which seems consistent with the XPS binding energies and the broad $\mathrm{OH}$-stretch vibrations that remained present. Moreover, the polydispersity of these particles is much larger than in the Ce-TTPMA system. Contrary to the Ce-TTPMA system, where phase separation seemed to occur during the calcination process, phase separation in the Ni-TTPMA system was probably initiated by the redistribution of malonamide ligands.

\subsubsection{Membrane characteristics of the Ni-TTPMA coated layer}

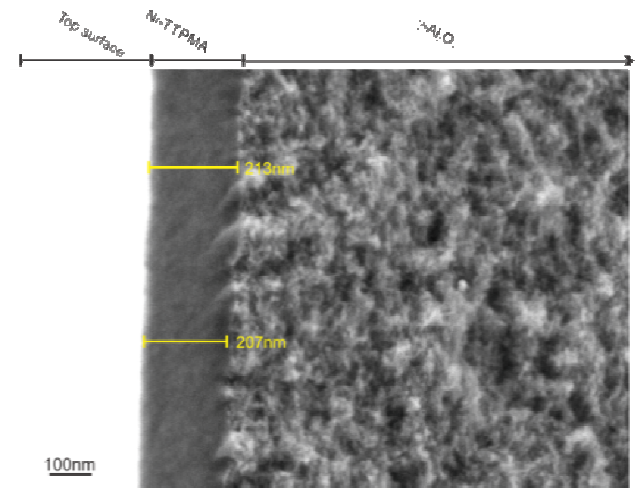

Figure 7.15. HR-SEM image of a selective Ni-TTPMA coated layer on a meso/macroporous $\quad \gamma-\mathrm{Al}_{2} \mathrm{O}_{3} / \alpha-\mathrm{Al}_{2} \mathrm{O}_{3}$ support.
A thin selective layer of approx. $210 \mathrm{~nm}$ thickness was formed on top of the mesoporous $\gamma$ $\mathrm{Al}_{2} \mathrm{O}_{3}$ layer after dipcoating the Ni-TTPMA sol, drying and calcination at $T=250^{\circ} \mathrm{C}$ (Figure 7.15). The thickness of the defect-free permselective membrane layer was similar to a previously described BTESE (1,2-bis-(triethoxysilyl)ethane) membrane [1]. Helium and hydrogen fluxes were one order of magnitude smaller than through hybrid silica (BTESE)

membranes. Thus, the selective layer was much more resistive than the BTESE membrane. This may have several causes: (1) narrower pores, (2) $\mathrm{Ni}_{2} \mathrm{O}_{3}$ particles that effectively block pores, (3) lower porosity and/or (4) by the presence of water that remained present in the structure. It has been demonstrated that remnant water in amino-functionalized silsesquioxane membranes reduced their gas permeability [84]. 


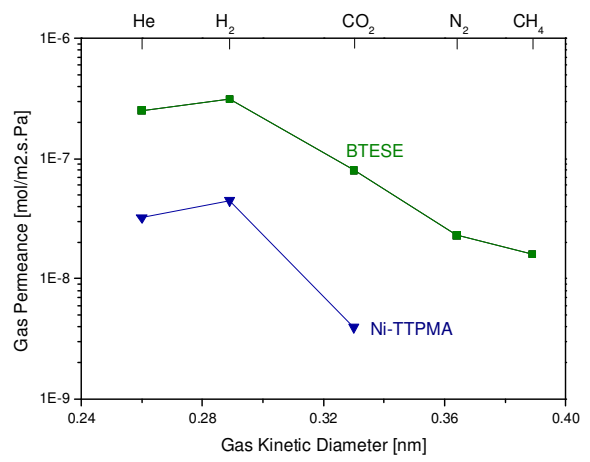

Figure 7.16 Single gas permeances of a Ni-TTPMA membrane layer on a meso/macroporous $\gamma-\mathrm{Al}_{2} \mathrm{O}_{3} / \alpha-\mathrm{Al}_{2} \mathrm{O}_{3}$ support, and a hybrid BTESEmembrane as reported elsewhere [1].
Remarkably, the membrane was not permeable to $\mathrm{N}_{2}$ so that it exhibited an infinitely high $\mathrm{H}_{2} / \mathrm{N}_{2}$ permselectivity. To our knowledge, $\mathrm{Ni}$ doping in any silica or any bridged silsesquioxane precursor has not yielded any nitrogen impermeable membrane to date. The high permselectivity can be attributed to a small pore size and a narrow pore size distribution. No indication was found for affinity between $\mathrm{Ni}$ species and $\mathrm{H}_{2}$. Most likely, the size of the $\mathrm{Ni}_{2} \mathrm{O}_{3}$ particles needs to be reduced further to measure a significant effect of affinity towards $\mathrm{H}_{2}$.

\subsection{Conclusions}

$N, N, N^{\prime}, N^{\prime}$-tetrakis-(3-(triethoxysilyl)-propyl)-malonamide (TTPMA) was synthesized successfully through a base catalyzed nucleophilic substitution of bis-(3-(triethoxysilyl)-propyl)-amine onto malonyl chloride. The malonamide ligand of TTPMA was found to coordinate both $\mathrm{Ce}^{4+}$ and $\mathrm{Ni}^{2+}$ of cerium isopropoxide and nickel nitrate hexahydrate, respectively.

In the case of $\mathrm{Ce}$-TTPMA $\mathrm{CeO}_{2}$ phase separated while calcining the mixed hybrid film at $T=250^{\circ} \mathrm{C}$. Consequently, nanosized clusters $(2-3 \mathrm{~nm})$ of $\mathrm{CeO}_{2}$ were formed. The single gas permeances revealed a slightly larger $\mathrm{H}_{2} / \mathrm{N}_{2}$ permselectivity as compared to a previously reported BTESE hybrid membrane, which was substantially thicker $(260 \mathrm{~nm})$ than the Ce-TTPMA layer.

The conformation of the Ni-TTPMA complex slowly evolves while aging the complex in ethanol. Consequently, some nickel nitrate could phase separate while drying the sol. After calcination nanosized (1-15 nm) nickel oxide particles (most likely $\mathrm{Ni}_{2} \mathrm{O}_{3}$ ) were observed by TEM. The $210 \mathrm{~nm}$ 
selective layer of Ni-TTPMA was approx. 1 order of magnitude more resistive towards gas permeation of $\mathrm{H}_{2}$, He and $\mathrm{CO}_{2}$ as compared to a hybrid BTESE layer of similar thickness [1]. No $\mathrm{N}_{2}$ permeance was observed within the experimental error, which makes this material a promising gasselective material for $\mathrm{CO}_{2} / \mathrm{N}_{2}$ separation.

\subsection{References}

1. Qureshi, H.F., A. Nijmeijer, and L. Winnubst, Influence of sol-gel process parameters on the micro-structure and performance of hybrid silica membranes. J. Membr. Sci., 2013. 446: p. 19-25.

2. Li, J.-R., et al., Carbon dioxide capture-related gas adsorption and separation in metal-organic frameworks. Coordin. Chem. Rev., 2011. 255(15-16): p. 1791-1823.

3. Shekhawat, D., D.R. Luebke, and H.W. Pennline, A review of carbon dioxide selective membranes. US Department of Energy, 2003.

4. Olah, G.A., A. Goeppert, and G.K.S. Prakash, Chemical Recycling of Carbon Dioxide to Methanol and Dimethyl Ether: From Greenhouse Gas to Renewable, Environmentally Carbon Neutral Fuels and Synthetic Hydrocarbons. J. Org. Chem., 2008. 74(2): p. 487-498.

5. Ho, M.T., et al., Economics of $\mathrm{CO} 2$ and Mixed Gas Geosequestration of Flue Gas Using Gas Separation Membranes. Ind. Eng. Chem. Res., 2005. 45(8): p. 2546-2552.

6. Chen, H., A.S. Kovvali, and K.K. Sirkar, Selective CO2 Separation from CO2-N2 Mixtures by Immobilized Glycine-Na-Glycerol Membranes. Ind. Eng. Chem. Res. 2000. 39(7): p. 2447-2458.

7. Granite, E.J. and T. O'Brien, Review of novel methods for carbon dioxide separation from flue and fuel gases. Fuel Processing Technology, 2005. 86(14-15): p. 1423-1434.

8. de Vos, R.M. and H. Verweij, Improved performance of silica membranes for gas separation. Journal of Membrane Science, 1998. 143(1-2): p. 37-51.

9. Kanezashi, M. and M. Asaeda, Hydrogen permeation characteristics and stability of Ni-doped silica membranes in steam at high temperature. J. Membr. Sci., 2006. 271(1-2): p. 86-93.

10. Igi, R., et al., Characterization of co-doped silica for improved hydrothermal stability and application to hydrogen separation 
membranes at high temperatures. J. Am. Ceram. Soc., 2008. 91(9): p. 2975-2981.

11. Buxbaum, R.E. and A.B. Kinney, Hydrogen transport through tubular membranes of palladium-coated tantalum and niobium. Ind. Eng. Chem. Res., 1996. 35(2): p. 530-537.

12. Qi, H., et al., Effect of $\mathrm{Nb}$ content on hydrothermal stability of a novel ethylene-bridged silsesquioxane molecular sieving membrane for H2/CO2 separation. J. Membr. Sci., 2012. 421-422(0): p. 190200.

13. Qi, H., et al., Hybrid Organic-Inorganic Microporous Membranes with High Hydrothermal Stability for the Separation of Carbon Dioxide. ChemSusChem, 2010. 3(12): p. 1375-1378.

14. Boffa, V., et al., Microporous niobia-silica membrane with very low CO2 permeability. ChemSusChem, 2008. 1(5): p. 437-443.

15. Kanezashi, M. and M. Asaeda, Stability of H2-permeselective Nidoped silica membranes in steam at high temperature. J. Chem Eng. Jpn., 2005. 38(11): p. 908-912.

16. Takaba, H., et al., The effect of gas molecule affinities on $\mathrm{CO} 2$ separation from the CO2/N2 gas mixture using inorganic membranes as investigated by molecular dynamics simulation. J. Membr. Sci., 1996. 121(2): p. 251-259.

17. Busca, G. and V. Lorenzelli, Infrared spectroscopic identification of species arising from reactive adsorption of carbon oxides on metal oxide surfaces. Mater. Chem., 1982. 7(1): p. 89-126.

18. Sakakura, T., J.-C. Choi, and H. Yasuda, Transformation of carbon dioxide. Chem. Rev., 2007. 107(6): p. 2365-2387.

19. Lan, J., et al., Doping of alkali, alkaline-earth, and transition metals in covalent-organic frameworks for enhancing $\mathrm{CO} 2$ capture by firstprinciples calculations and molecular simulations. ACS nano, 2010. 4(7): p. 4225-4237.

20. Uhlhorn, R.J.R., K. Keizer, and A.J. Burggraaf, Gas and surface diffusion in modified $\gamma$-alumina systems. J. Membr. Sci., 1989. 46(23): p. 225-241.

21. Busca, G., The surface Acidity and Basidity of Solid Oxides and Zeolites, in Metal Oxides, Chemistry and Apllications, F.J.L. G., Editor 2006, CRC Press Taylor \& Francis Group: Boca Raton. p. 247-318.

22. Bordes-Richard, E.C., P., Optical basicity: A scale of Acidity/Basicity of Solids and its Application to Oxidation Catalysis, in Metal Oxides Chemistry and applications, J.L.G. Fierro, Editor 2006, CRC press Taylor \& Francis Group: New York. p. 319-352. 
23. Appel, L.G., J.G. Eon, and M. Schmal, The CO2-CeO2 interaction and its role in the $\mathrm{CeO} 2$ reactivity. Catal. Lett., 1998. 56(4): p. 199-202.

24. Du, X., et al., Diffuse reflectance infrared Fourier transform and Raman spectroscopic studies of $\mathrm{MoO} 3$ dispersed on $\mathrm{CeO} 2$ support. Langmuir, 1999. 15(5): p. 1693-1697.

25. Gaudon, A., et al., Phase separation in sol-gel derived ZrO2-SiO2 nanostructured materials. J. Eur. Ceram. Soc., 2005. 25(2-3 SPEC. ISS.): p. 283-286.

26. Kessler, V.G., et al., New insight in the role of modifying ligands in the sol-gel processing of metal alkoxide precursors: A possibility to approach new classes of materials. J. Sol-Gel Sci. Technol., 2006. 40(2-3): p. 163-179.

27. Brinker, C.J.S., G. W. , Sol Gel Science, The Physics and Chemistry of Sol-Gel Processing. Vol. 1. 1990, San Diego: Acedemic Press. 1-881.

28. Sanchez, C., et al., Chemical modification of alkoxide precursors. J. Non-Cryst. Solids, 1988. 100(1-3): p. 65-76.

29. Sedlar, M. and M. Sayer, Reactivity of titanium isopropoxide, zirconium propoxide and niobium ethoxide in the system of 2methoxyethanol, 2,4-pentanedione and water. J. Sol-Gel Sci. Technol., 1995. 5(1): p. 27-40.

30. Percy, M., et al., The influence of 6 -diketones on the induction times for hydrolysis of zirconium (IV) alkoxides. J. Mater. Chem., 1999. 9(2): p. 499-505.

31. Rupp, W., N. Hüsing, and U. Schubert, Preparation of silica-titania xerogels and aerogels by sol-gel processing of new single-source precursors. J. Mater. Chem., 2002. 12(9): p. 2594-2596.

32. Puchberger, M., et al., Reaction of metal alkoxides with 3-alkylsubstituted acetylacetone derivatives-coordination vs. hydrodeacylation. New J. Chem., 2004. 28(11): p. 1289-1294.

33. Peterlik, H., et al., Structural investigation of alumina silica mixed oxide gels prepared from organically modified precursors. Journal of Non-Crystalline Solids, 2007. 353(16-17): p. 1635-1644.

34. Dubois, G., et al., Molecular Network Reinforcement of Sol-Gel Glasses. Adv. Mater., 2007. 19(22): p. 3989-3994.

35. Dubois, G., et al., Superior mechanical properties of dense and porous organic/inorganic hybrid thin films. J. Sol-Gel Sci. Technol., 2008. 48(1-2): p. 187-193.

36. Oliver, M.S., et al., Molecular Origins of the Mechanical Behavior of Hybrid Glasses. Adv. Funct. Mater., 2010. 20(17): p. 2884-2892. 
37. Tian, Q. and M.A. Hughes, Synthesis and characterisation of diamide extractants for the extraction of neodymium. Hydrometallurgy, 1994. 36(1): p. 79-94.

38. Kudo, H., F. Sanda, and T. Endo, Efficient synthesis of macrocycles by oxidation of cysteine-based dithiols. Tetrahedron Lett., 2001. 42(44): p. 7847-7850.

39. Ellis, R.J. and M.R. Antonio, Coordination Structures and Supramolecular Architectures in a Cerium(III)-Malonamide Solvent Extraction System. Langmuir, 2012. 28(14): p. 5987-5998.

40. Ellis, R.J., et al., Solvent extraction of cerium (III) using an aliphatic malonamide: The role of acid in organic phase behaviors. Separation Science and Technology, 2012. 47(14-15): p. 2007-2014.

41. Parks, B.W., et al., Solution and Structural Investigations of Ligand Preorganization in Trivalent Lanthanide Complexes of Bicyclic Malonamides. Inorganic chemistry, 2006. 45(4): p. 1498-1507.

42. Lumetta, G.J., et al., Synthesis and characterization of mono- and bis-(tetraalkylmalonamide)uranium(VI) complexes. Inorganica Chimica Acta, 2000. 309(1-2): p. 103-108.

43. Chan, G.Y., et al., Solvent extraction of metal ions from nitric acid solution using $N, N^{\prime}$-substituted malonamides. Experimental and crystallographic evidence for two mechanisms of extraction, metal complexation and ion-pair formation. Journal of the Chemical Society, Dalton Transactions, 1997(4): p. 649-660.

44. Drew, M.G., et al., Crystal structure of [Yb (L (NO3) 2 (H2O) 2](NO3), $L=$ bromo- $N, N, N^{\prime}, N^{\prime}$-tetraethylmalonamide. Journal of Chemical Crystallography, 2000. 30(7): p. 455-458.

45. Fedosseev, A.M., et al., Synthesis, crystal structure and some properties of new perrhenate and pertechnetate complexes of $\mathrm{Nd} 3+$ and Am3+ with 2,6-bis(tetramethylfurano)-1,2,4-triazin-3-yl)pyridine, tris(2-pyridylmethyl)amine and $N, N^{\prime}$ tetraethy/malonamide. Polyhedron, 2008. 27(8): p. 2007-2014.

46. Iveson, P.B., et al., Structural studies of lanthanide complexes with new hydrophobic malonamide solvent extraction agentst. Journal of the Chemical Society, Dalton Transactions, 1999(20): p. 36053610.

47. Parella, T., Pulse Program Catalogue. NMR Guide, 2004. 4.

48. Poppe, L. and $\mathrm{H}$. van Halbeek, $13 \mathrm{C}$-selective, $1 \mathrm{H}$-detected $2 \mathrm{D}\{1 \mathrm{H}$, 13C correlation spectra of oligosaccharides. Magnetic Resonance in Chemistry, 1991. 29(8): p. 848-851. 
49. Bax, A. and M.F. Summers, Proton and carbon-13 assignments from sensitivity-enhanced detection of heteronuclear multiple-bond connectivity by $2 D$ multiple quantum NMR. Journal of the American Chemical Society, 1986. 108(8): p. 2093-2094.

50. Hurd, R.E. and B.K. John, Gradient-enhanced proton-detected heteronuclear multiple-quantum coherence spectroscopy. J. Mag. Reson. (1969), 1991. 91(3): p. 648-653.

51. De Vos, R.M. and H. Verweij, High-selectivity, high-flux silica membranes for gas separation. Science, 1998. 279(5357): p. 17101711.

52. Cao, G.Z., et al., Permporometry study on the size distribution of active pores in porous ceramic membranes. J. Membr. Sci., 1993. 83(2): p. 221-235.

53. $N, N, N^{\prime}, N^{\prime}$-tetrapropylmalonamide (SDBS-no= 51018), Available from SDBSWeb: http://sdbs.riodb.aist.go.jp, (National Institute of Advanced Industrial Science and Technology (AIST)), accessed on 14-05-2013.

54. Bradley, E.K., et al., NMR structural characterization of oligo-Nsubstituted glycine lead compounds from a combinatorial library. Mol. Divers., 1997. 3(1): p. 1-15.

55. Bradley, E.K., A Method for Sequential NMR Assignment of ${ }^{1} \mathrm{H}$ and ${ }^{13} \mathrm{C}$ Resonances of N-Substituted Glycine Peptoids. J. Mag. Reson. B, 1996. 110(2): p. 195-197.

56. Bax, A., S.W. Sparks, and D.A. Torchia, Long range heteronuclear correlation. A powerful tool for the NMR analysis of medium-size proteins. J. Am. Chem. Soc., 1988. 110(23): p. 7926-7927.

57. X.Webster, R.M.S.F., Spectrometric identification of organic compunds. 6th ed.1998, New York: John Wiley \& Sons, Inc.

58. ethyl 2-chloro-3-oxobutyrate (SDBS-no= 12496). Available from SDBSWeb: http://sdbs.riodb.aist.go.jp (National Institute of Advanced Industrial Science and Technology (AIST)) accessed on 1405-2013.

59. diethyl 3-oxoglutarate (SDBS-No=6503). Available from SDBSWeb: http://sdbs.riodb.aist.go.jp, (National Institute of Advanced Industrial Science and Technology (AIST)), accessed on 14-05-2013.

60. Ryu, Y. and A.I. Scott, Self-condensation of activated malonic acid half esters: a model for the decarboxylative Claisen condensation in polyketide biosynthesis. Tetrahedron Lett., 2003. 44(40): p. 74997502. 
61. Den Auwer, C., et al., Crystallographic, X-ray Absorption, and IR Studies of Solid- and Solution-State Structures of Tris(nitrato) $N, N, N^{\prime}, N^{\prime}$-Tetraethylmalonamide Complexes of Lanthanides. Comparison with the Americium Complex. Inorg. Chem., 2000. 39(7): p. 1487-1495.

62. Fleeting, K.A., et al., Studies on Mixed B-Diketonate/Isopropoxide Compounds of Zirconium and Hafnium, the X-ray Single-Crystal Structures of [M2(OPri)6(tmhd)2] $(M=Z r, H f)$ : Some Chemistry Important in the MOCVD of Oxides. Inorg. Chem., 1999. 38(7): p. 1432-1437.

63. Vaartstra, B.A., et al., Alcohol adducts of alkoxides: intramolecular hydrogen bonding as a general structural feature. Inorg. Chem., 1990. 29(17): p. 3126-3131.

64. Castricum, H.L., et al., Hydrothermally stable molecular separation membranes from organically linked silica. J. Mater. Chem., 2008. 18(18): p. 2150-2158.

65. Bo, L., et al., High-pressure Raman study on Ce O2 nanospheres selfassembled by $5 \mathrm{~nm}$ Ce $\mathrm{O} 2$ nanoparticles. Phys Status Solidi B: 2011. 248: p. 1154-1157.

66. Bickley, R.I., et al., Vibrational spectroscopic study of nickel(II) hypophosphite, Ni(H2PO2)2. J. Mol. Struct., 1992. 273: p. 61-72.

67. Nakamoto, K., Infra red and Raman spectra of Inorganic and coodination compounds. 4 ed, ed. K. Nakamoto 1986, New York: John Whiley \& Sons. 259-263.

68. Matos, M., L. Ilharco, and R. Almeida, The evolution of TEOS to silica gel and glass by vibrational spectroscopy. J. Non-Cryst. Solids, 1992. 147: p. 232-237.

69. Niznansky, D. and J. Rehspringer, Infrared study of SiO< sub> $2</ s u b>$ sol to gel evolution and gel aging. J. Non-Cryst. Solids, 1995. 180(2): p. 191-196.

70. Chen, S.G., Y.S. Yin, and D.P. Wang, Formation of Ring-Like Si-O-Zr Bonds at Intergranular Interfaces in Silica-Doped Zirconia. J. Am. Ceram. Soc., 2005. 88(4): p. 1041-1045.

71. Tejedor-Tejedor, M.I., L. Paredes, and M.A. Anderson, Evaluation of ATR-FTIR Spectroscopy as an "in Situ" Tool for Following the Hydrolysis and Condensation of Alkoxysilanes under Rich $\mathrm{H} 2 \mathrm{O}$ Conditions. Chem. Mater., 1998. 10(11): p. 3410-3421.

72. Niven, M.L. and G.C. Percy, The infrared spectra $(3500-140 \mathrm{~cm}-1)$ of the 2, 2'-bipyridine, 2-aminomethylpyridine and ethylenediamine adducts and the sodium tris-compounds of cobalt (II), nickel (II) and 
zinc (II) acetylacetonates. Transit. Metal Chem., 1978. 3(1): p. 267271.

73. Castricum, H.L., et al., Microporous structure and enhanced hydrophobicity in methylated SiO 2 for molecular separation. J. Mater. Chem., 2007. 17(15): p. 1509-1517.

74. de Vos, R.M., W.F. Maier, and H. Verweij, Hydrophobic silica membranes for gas separation. J. Membr. Sci., 1999. 158(1-2): p. 277-288.

75. Brockner, W., C. Ehrhardt, and M. Gjikaj, Thermal decomposition of nickel nitrate hexahydrate, $\mathrm{Ni}(\mathrm{NO} 3) 2 \cdot 6 \mathrm{H} 2 \mathrm{O}$, in comparison to $\mathrm{Co}(\mathrm{NO} 3) 2 \cdot 6 \mathrm{H} 2 \mathrm{O}$ and $\mathrm{Ca}(\mathrm{NO} 3) 2 \cdot 4 \mathrm{H} 2 \mathrm{O}$. Thermochim. Acta, 2007. 456(1): p. 64-68.

76. Mansour, S.A.A., Spectroscopic and microscopic investigations of the thermal decomposition of nickel oxysalts: Part 2. Nickel nitrate hexahydrate. Thermochim. Acta, 1993. 228(0): p. 173-189.

77. Mansour, A., Characterization of NiO by XPS. Surf. Sci. Spectra, 1994. 3: p. 231.

78. de Souza, M.O., et al., XPS characterization of nickelacetylacetonate impregnated in NaX and NaY zeolites. Micropor. Mesopor. Mat., 2004. 69(3): p. 217-221.

79. Ho, S.-C. and T.-C. Chou, The Role of Anion in the Preparation of Nickel Catalyst Detected by TPR and FTIR Spectra. Ind. Eng. Chem. Res., 1995. 34(7): p. 2279-2284.

80. Lian, K., D. Kirk, and S. Thorpe, Investigation of a "Two-State" Tafel Phenomenon for the Oxygen Evolution Reaction on an Amorphous Ni-Co Alloy. J. Electrochem. Soc., 1995. 142(11): p. 3704-3712.

81. Zhao, G., et al., Ni2O3-Au+ hybrid active sites on NiOx@Au ensembles for low-temperature gas-phase oxidation of alcohols. Catal. Sci. Technol., 2013. 3(2): p. 404-408.

82. Salvati, L., et al., Surface spectroscopic study of tungsten-alumina catalysts using $x$-ray photoelectron, ion scattering, and Raman spectroscopies. J. Phys. Chem., 1981. 85(24): p. 3700-3707.

83. Dufresne, P., et al., Study of nickel-molybdenum-gamma.aluminum oxide catalysts by $x$-ray photoelectron and Raman spectroscopy. Comparison with cobalt-molybdenum-.gamma.aluminum oxide catalysts. J. Phys. Chem., 1981. 85(16): p. 23442351.

84. Paradis, G.G., et al., Amino-functionalized microporous hybrid silica membranes. J. Mater. Chem., 2012. 22(15): p. 7258-7264. 
Conclusions and Outlook 


\subsection{General Conclusions}

The hydrolysis and condensation of silsesquioxanes cannot compete with the fast kinetics of metal alkoxides such as niobium penta ethoxide (NPE). Consequently, the speed of nucleation of NPE was scarcely affected by the presence of a silsesquioxane: 1,2-bis-(triethoxysilyl)-ethane (BTESE) (Chapter3). Nevertheless, subsequent growth was affected by the presence of BTESE as indicated by a reduced growth exponent and reduced massfractal dimension. Growth of BTESE/NPE sols most likely occurred through reversible Lifshitz-Slyozov type agglomeration kernels unlike growth in the absence of BTESE that most likely occurred through irreversible Smoluchowski type agglomeration kernels.

During the initial stages of the drying process of BTESE/NPE sols the size of primary building blocks increased in size while the overall agglomerate size remained almost constant (Chapter 4). This could be associated with a coarsening effect of branched particles [1, 2]. This agrees with LifshitzSlyozov type agglomeration, since irreversible agglomeration kernels would not allow structural rearrangements. During the next stage of the drying process electron rich domains became apparent that were associated with phase separated niobia. The size of phase separated niobia was reduced when BTESE was hydrolysed first before adding NPE.

Nevertheless, following the ${ }^{17}$ O-NMR spectroscopy (Chapter 5) the prehydrolysis of BTESE before adding NPE did not led to a substantial increase in the number of Si-O-Nb linkages. On the other hand the use of acetylacetone that effectively blocked coordination sites of NPE led to a substantial increase in the number of Si-O-Nb linkages. Moreover ${ }^{29} \mathrm{Si}-\mathrm{NMR}$ proved the survival of the Si-C linkage up to $T=200^{\circ} \mathrm{C}$ in air.

$\mathrm{Nb}_{8} \mathrm{O}_{10}(\mathrm{OEt})_{20}$ could be synthesized through a slow and controlled condensation of niobium(V)ethoxide with acetone as a oxolation source at room temperature (Chapter 6 ). These recovered $\mathrm{Nb}_{8} \mathrm{O}_{10}(\mathrm{OEt})_{20}$ crystallites were found to exhibit a remarkable anisotropic thermal expansion coefficient. 
$N, N, N^{\prime}, N^{\prime}$-tetrakis-(3-(triethoxysilyl)-propyl)-malonamide (TTPMA) was synthesized successfully through a base catalyzed nucleophilic substitution of bis-(3-(triethoxysilyl)-propyl)-amine onto malonyl chloride (Chapter 7). The malonamide ligand of TTPMA was found to coordinate both $\mathrm{Ce}^{4+}$ and $\mathrm{Ni}^{2+}$ of cerium isopropoxide and nickel nitrate hexahydrate, respectively. However, phase separation of both $\mathrm{CeO}_{2}$ and $\mathrm{NiO}$ was not completely avoided. Despite the phase separated metal oxide clusters single gas permeation measurements gave reasonable results: Although, the $\mathrm{Ce}$ TTPMA and Ni-TTPMA the layers were more resistant to gas permeation as compared to previously measured BTESE membranes [3], they also became more selective.

\subsection{Additional observations and remarks}

This paragraph described additional results that were not incorporated in one of the previous chapters.

Nonhydrolytic Sol-Gel Synthesis: These synthesis were expected to yield $\mathrm{Nb}-\mathrm{O}-\mathrm{SI}$ more selectively. However, several different non-hydrolytic ester elimination and ethyl halide elimination reactions were verified at $T=110^{\circ} \mathrm{C}$ which includes: (1) Ester elimination between niobium penta ethoxide and di-t-butoxy-di-acetoxysilane in dichloromethane, (2) Ethyl halide elimination niobium penta ethoxide and 1,2-bis-(trichlorosilyl)-ethane in dichloromethane and (3) Ethyl halide elimination niobium penta chloride and 1,2-bis-(triethoxysilyl)-ethane in tetrahydrofuran. H-NMR results indicated that ligand exchange easily occurred, which enabled a faster condensation of metal alkoxide as compared to the silicon alkoxide. In conclusion, although the condensation rates of niobium ethoxide may be reduced through a nonhydrolytic pathways, it did not resolve the mismatch in condensation rates between silicon and metal alkoxides. 
Noesy-correlations TTPMA-NPE complex: By means of the nuclear Overhauser effect cross-correlations can be measured between protons that are in a close surrounding to each other ( $\sim 5 \AA$ in distance) [4]. Hence, it can confirm cross-correlation between protons of a metal alkoxide that engaged protons of the $N, N, N^{\prime}, N^{\prime}$-tetrakis-(3-(triethoxysilyl)-propyl)malonamide (TTPMA) molecule upon complexation. After mixing a mixture of niobium penta ethoxide (NPE) with TTPMA in a 1:1 ratio for one day a noesygpphpp [5] was performed in $\mathrm{CDCl}_{3}$ (Figure 8.1a). Clear crosscorrelations were observed between particular $\mathrm{CH}_{2}$ entities of NPE and $\mathrm{CH}_{2}$ entities of the propylene bridge of TTPMA as being assigned by the symbols (I) and (II). These assignments were schematically illustrated in Figure 8.1b. The exact nature of this complex was difficult to resolve and several entities coexisted in a range between $4.0<\delta<4.5$.

To confirm Ce-TTPMA complexation as being discussed in Chapter 7 noesy experiments on such mixtures may be useful as well. However, NMRspectroscopy measurements of Cerium(IV)isopropoxide did not gave reliable results, since no cross-correlations were found in a ${ }^{1} \mathrm{H},{ }^{1} \mathrm{H}$-Cosy experiments. This may be a consequence of paramagnetic impurities.

\subsection{Future perspective and outlook}

In the last decennia, the chemistry of metal alkoxide / silicon alkoxide mixtures have been exploited to a large extent. The extent of phase separation can be reduced to certain extent but it rarely completely avoided. Therefore, this direction does not offer much possibilities anymore. On the other hand the hybrid chemistry of silsesquioxanes still offer many opportunities, since the many combinations of organic chemistry approaches with silicon alkoxide chemistry are far from being exploited: 

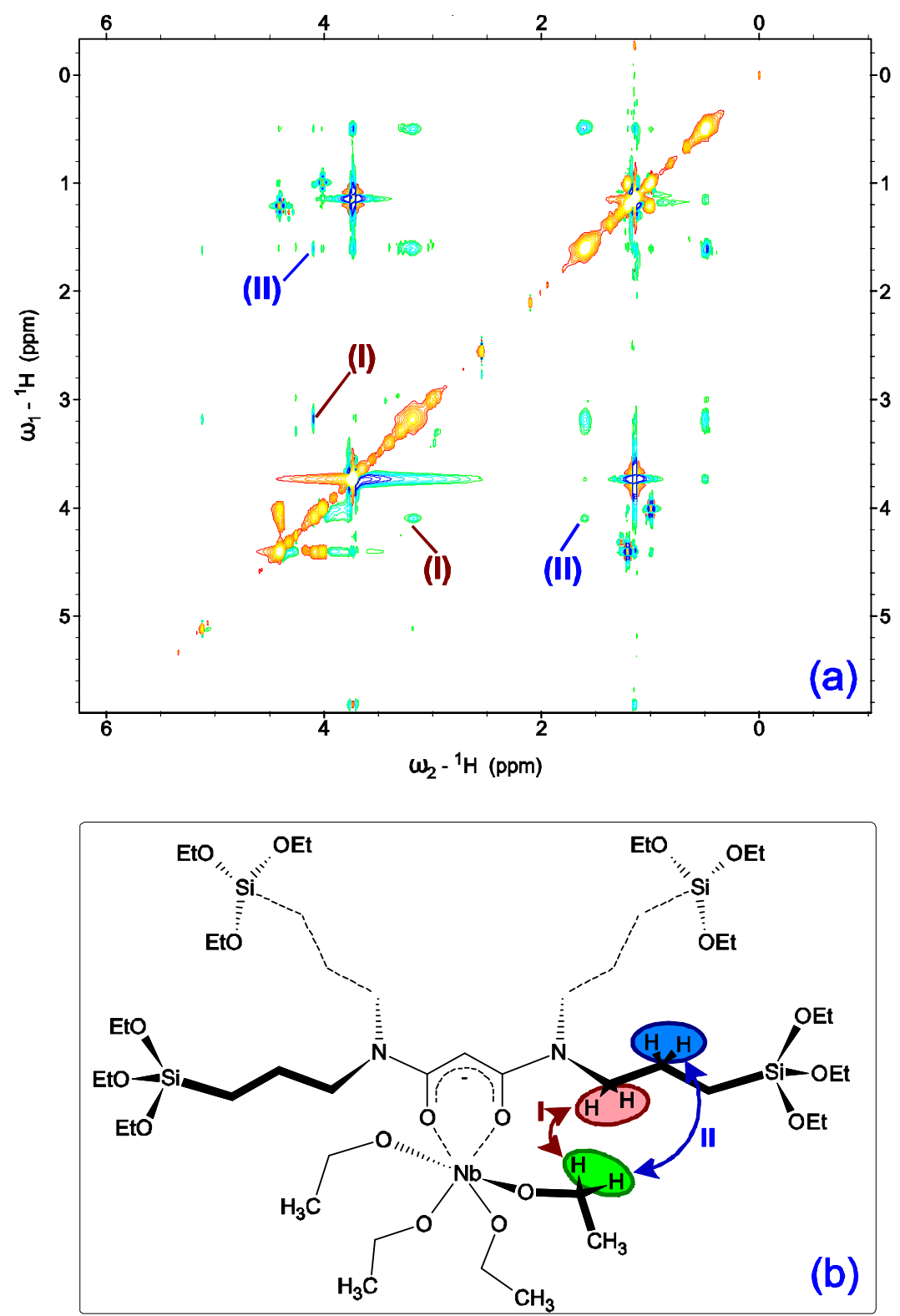

Figure 8.1. (a) ${ }^{1} \mathrm{H},{ }^{1} \mathrm{H}$-noesy experiment of an 1:1 NPE:TTPMA mixture in $\mathrm{CDCl}_{3}$. (b) Molecular illustration of cross-correlation between protons of NPE and TTPMA in the ${ }^{1} \mathrm{H},{ }^{1} \mathrm{H}$-noesy experiment (a). 
Hydrothermal and chemical stability: A higher hydrothermal and chemical stability may be achieved by further engaging the boundary between ceramics and polymers. Thus, replacing the bridged silsesqioxane precursor that contained $2 \mathrm{x}-\mathrm{Si}(\mathrm{OR})_{3}$ entities for small oligomers that contained $3-20 \mathrm{x}$ $-\mathrm{Si}(\mathrm{OR})_{3}$ entities. The highest network stability is probably achieved by a smart combination of different chemical bonds that build-up the network. For instance by combining amide-bonds that are more resistant to bases with siloxane-bonds that are more resistant to acids. Consequently, attack of either one of them would have a lower impact.

Alter the membrane's affinity for particular gases or molecules: Silsesquioxanes can be attached to organometalic entities such as (1) porphyrins, or (2) metaloscenes. One very famous porphyrin: heme is responsible for the transport of oxygen and carbon dioxide through the human body.

\subsection{References}

1. Conti, M., A. Lipshtat, and B. Meerson, Scaling anomalies in the coarsening dynamics of fractal viscous fingering patterns. Physical Review E, 2004. 69(3): p. 031406.

2. Sharon, E., et al., Coarsening of Fractal Viscous Fingering Patterns. Physical review letters, 2003. 91(20): p. 205504.

3. Qureshi, H.F., A. Nijmeijer, and L. Winnubst, Influence of sol-gel process parameters on the micro-structure and performance of hybrid silica membranes. Journal of Membrane Science, 2013. 446(0): p. 19-25.

4. Anet, F.A.L. and A.J.R. Bourn, Nuclear Magnetic Resonance Spectral Assignments from Nuclear Overhauser Effects1. Journal of the American Chemical Society, 1965. 87(22): p. 5250-5251.

5. Parella, T., Pulse Program Catalogue. NMR Guide, 2004. 4. 
Summary. 
Chapter 1 Introduces the Sol-Gel Chemistry of silicon and metal alkoxides and the fundamental differences between them. In addition strategies to manipulate their chemistry were being discussed. Then, it continues with by describing the processes being involved to make microporous membranes. This is being followed by a description of microporous membranes and their transport mechanisms. Then, this chapter was finalized by describing the scope of the thesis with a brief outline.

Chapter 2 evaluated theoretical aspects of Small Angle X-ray Scattering (SAXS), which are useful for the understanding of Chapter $\mathbf{3}$ and Chapter $\mathbf{4}$ where this technique was evaluated extensively. It evaluated the basic concepts of form and structure functions for mass-fractalic and correlated systems with the main focus on isotropically dispersed spherical like agglomerates.

Chapter 3 involved rapid in-situ SAXS measurements were performed to measure early-stage agglomeration processes under niobium ethoxide mixtures in the presence and absence of 1,2-bis-(triethoxysilyl)-ethane (BTESE). A newly derived mass-fractalic model was used that allowed the characterisation of the radii of gyration $\left(R_{\mathrm{G}}\right)$, mass-fractalic dimension $\left(D_{\mathrm{f}}\right)$ and Z-parameter that was associated with the poydispersity. The trends of the experiments in the presence and absence of BTESE were consistent diffusion limited cluster aggregation. Moreover the growth processes led to self-preserving polydispersities. Nevertheless, growth in the absence of BTESE was consistent with irreversible Smoluchowski type agglomeration kernels, while growth in the presence of BTESE was more consistent with reversible Lifshitz-Slyozov type agglomeration kernels.

Chapter 4 evaluated in-situ SAXS measurements while drying mixed niobium ethoxide bridged silsesquioxane sol at $T=40-80^{\circ} \mathrm{C}$. During this drying process the system changed from a system that containes branched polymer-like particles into a system with correlated domains. The correlated domains were associated with nanosized electron rich niobia clusters that were embedded inside an condensed silsesquioxane matrix. Therefore, these SAXS-measurements could be used to minimize the size of the phase-separated niobia domains. When the silsesquioxane precursor (1,2-bis-triethoxysilyl)-ethane) was hydrolysed first before addition of niobium penta ethoxide slightly smaller niobia particles had phase 
separated as compared to the case when both precursors were hydrolyzed simultaneously.

In Chapter 5 different synthesis routes were evaluated with the aim of optimizing the incorporation of niobium within a hybrid silica matrix on an atomic scale. Due to mismatch in hydrolysis/condensation rates between 1,2-bis-triethoxy-ethane (BTESE) and niobium penta-ethoxide (NPE), phase separated domains of $\mathrm{Nb}_{2} \mathrm{O}_{5}$ inside the silsesquioxane matrix were expected. To overcome this effect we a) performed a prehydrolysis of BTESE prior to adding NPE, or b) attempted to reduce the availability of $\mathrm{Nb}$ via a complexation of $\mathrm{Nb}$ by either acetyl acetone or 2-methoxy-ethanol. The network organization was evaluated from results of FTIR as well as ${ }^{13} \mathrm{C}$, ${ }^{29} \mathrm{Si}$ and ${ }^{17} \mathrm{O}$ MAS NMR spectroscopy. Whereas the prehydrolysis of BTESE and the addition of 2-methoxy-ethanolinduced a but only moderate mixing of $\mathrm{Nb}$ and $\mathrm{Si}$, leading to a network in which islands of $\mathrm{Nb}_{2} \mathrm{O}_{5}$ are linked to the hosting silica based matrix via $\mathrm{Nb}-\mathrm{O}-\mathrm{Si}$ bonds, the use of acetylacetonate lead to a mixing of $\mathrm{Nb}$ and $\mathrm{Si}$ on the atomic scale, producing a mixed $\mathrm{Nb}-\mathrm{O}-\mathrm{Si}$ network without any extended clusters of segregated $\mathrm{Nb}_{2} \mathrm{O}_{5}$.

In Chapter 6 Acetone was evaluated as an oxolation source of niobium penta ethoxide to synthesize nanosized $\mathrm{Nb}_{8} \mathrm{O}_{10}(\mathrm{OEt})_{10}$ clusters. Its mild reactivity allows a controlled condensation and crystallization of this molecule. A process occurs via two complementary routes: (1) aldol condensation and (2) ether elimination. The structure refinement on the crystallites at $T=150 \mathrm{~K}$ and $T=296 \mathrm{~K}$ revealed a remarkable anisotropy in the thermal expansion of $\mathrm{Nb}_{8} \mathrm{O}_{10}(\mathrm{OEt})_{10}$ with a negative expansion coefficient along the $c$-axis. At reduced temperatures the $\mathrm{Nb}_{8} \mathrm{O}_{10}(\mathrm{OEt})_{10}$ clusters were tilted towards the $\underline{a}$ and $c$-axis, most likely driven by a reduction of thermal motion and steric hindrance. Moreover, the irregular shape of the clusters allowed a relatively large compression along the $a$-axis and a relatively large expansion along the $c$-axis.

In Chapter $\mathbf{7}$ we applied a different approach to the dispersion of metal ions within the silsesquioxane matrix: With the aim to synthesize single source precursors a malonamide bridged silsesquioxane was synthesized. These malonamide ligands were able to coordinate $\mathrm{Ce}^{4+}$ and $\mathrm{Ni}^{2+}$ metal centers and enhanced their dispersion. However, during calcination these metal centers redistributed into small nanosized grains of $\mathrm{CeO}_{2}(<5 \mathrm{~nm})$ and $\mathrm{Ni}_{2} \mathrm{O}_{3}$ 
( $<15 \mathrm{~nm}$ ). Nevertheless, these Ce-TTPMA and Ni-TTPMA membranes showed higher $\mathrm{H}_{2} / \mathrm{N}_{2}$ perselectivities as compared to previously reported hybrid BTESE (1,2-bis-(triethoxysilyl)ethane) membranes [1]. The TTPMAprecursor was found suitable for membrane separation and can be a promising and versatile precursor for the incorporation of metal ions within hybrid silica matrices. 


\section{Samenvatting.}


Dit proefschrift beschrijft een aantal methoden om metaalionen / metaaloxiden in microporeuze hybride silicaat glazen te verwerken om daarmee zijn eigenschappen aan te manipuleren. Een microporeus glas kan worden gezien als een wanordelijk web van atomen, waar minuscule poriën tussen gevormd zijn. Deze poriën zijn net groot genoeg om de kleinste moleculen zoals, koolzuurgas $\left(\mathrm{CO}_{2}\right)$, stikstof $\left(\mathrm{N}_{2}\right)$, waterstof $\left(\mathrm{H}_{2}\right)$, water $\left(\mathrm{H}_{2} \mathrm{O}\right)$, helium $(\mathrm{He})$, methanol $\left(\mathrm{CH}_{3} \mathrm{OH}\right)$ en methaan $\left(\mathrm{CH}_{4}\right)$ door the laten. Zodoende is het bruikbaar als een moleculaire filter waarbij kleine moleculen als waterstof $(\mathrm{d}=2.9 \AA)$ makkelijker door het membraan gaan als een molecuul dat net wat groter is zoals stikstof $(d=3.6 \AA ̊)$. Met het incorporeren van bepaalde metaalionen zouden we ten eerste de chemische stabiliteit kunnen verhogen om daarmee het materiaal meer corrosiebestendig te maken, en ten tweede de affiniteit voor bepaalde gassen kunnen manipuleren zodat bepaalde gassen gemakkelijker door het membraan migreren als anderen. Echter, het inbrengen van metaalionen is niet eenvoudig, daar zij zich meestal niet goed inmengen in de silica / hybride silica structuur en zich afscheiden als dichte metaaloxide clusters. Om die reden werd er ten eerste verscheidene stadia van deze syntheses bestudeerd waarin dit soort afscheidingen plaatsvinden, en werden er ten tweede alternatieve methoden toegepast om dit soort verschijnselen te onderdrukken.

Dit proefschrift start met een inleiding in chemie en processen die plaatsvinden voor de fabricage van dergelijke type membranen gevolgd door een beschrijving van de eigenschappen en transportprocessen in deze membranen (hoofdstuk 1). Vervolgens word er in hoofdstuk 2 een theoretische beschrijving gegeven voor de interpretatie van kleine hoek röntgen verstrooiing (SAXS) dat ons in staat stelt deeltjesgroei, agglomeratie en faseseparatie in situ te volgen gedurende mix, reflux en droogprocessen. Deze theorie is vervolgens toegepast in de experimentele hoofdstukken 3 en 4. Daarin beschrijft hoofdstuk 3 de eerste groeistadia van de niobium(V)ethoxide (NPE) met en zonder de aanwezigheid van een hybride silica precursor 1,2-bis-(triethoxysilyl)-ethane (BTESE). Hoewel BTESE nagenoeg een invloed lijkt te hebben op het snelle nucleatieproces van NPE, word daarentegen het groeiproces duidelijk veranderd. In tegenstelling tot de situatie zonder BTESE gaat deeltjes groei over naar een reversibel agglomeratieproces. Dit leidt op zijn beurt tot een lagere massafractale dimensie wat overeenkomt met een meer open structuur. 
Vervolgens richt hoofdstuk 4 zich op een later stadium van het fabricageproces: het droogproces. Dit proces was gekenmerkt door in drie stadia: (I) een stadium van verruwing of Oswald ripening waarbij de kleinste entiteiten uit het systeem blijken te verdwijnen, (II) een overgangsproces naar een gel/vaste stof, en (III) een verdichtingproces. Met name gedurende het overgangsproces (II) vind er een radicale verandering plaats in het verstrooiingspatroon, waarbij een correlatiepiek verschijnt. Een correlatie piek dat geassocieerd kan worden met fasegesepareerde metaal oxide clusters. Een zorgvuldige analyse van deze patronen geeft informatie betreffende de grote van dergelijke type clusters. Daarbij blijkt een voortijdige hydrolyse van de BTESE-precursor een kleine reductie van fasegesepareerde clusters op te leveren.

In het opvolgende hoofdstuk $\mathbf{5}$ werden de gedroogde glasachtige NPEBTESE poeders geanalyseerd met vaste stof kernspinresonantie spectroscopie. Dit stelde ons in staat iets meer te zeggen over de moleculaire verbindingen die in het systeem gevormd waren. In het bijzonder de kernspinresonantiemetingen op het ${ }^{17} \mathrm{O}$ met ${ }^{17} \mathrm{O}$ verrijkte samples gaven een goed inzicht in de dispersie van niobium ionen in de glas-achtige matrix. Daaruit bleek met name dat het gebruik van het afschermende legand acetylacetone een toenemende mate dispersie van de niobium ionen in de hybride silica matrix tot gevolg had.

In hoofdstuk 6 zijn de nonhydrolytische hydrolyse en condensatie van NPE onderzocht met acetone als een initiator in plaats van water. Een mildere initiator voor het condensatieproces leidt tot een tragere beter controleerbare condensatie van NPE dat in de afwezigheid van BTESE uitkristalliseerde tot vrij zuivere $\mathrm{Nb}_{8} \mathrm{O}_{10}(\mathrm{OEt})_{20}$ kristallen. Hoewel deze kristallen al eens eerder waren gesynthetiseerd, werd er dit keer een opmerkelijke thermische anisotropie waargenomen wat uitvoerig in het hoofdstuk beschreven is. In de aanwezigheid van BTESE bleken dit type condensatie mechanismen echter niet te werken.

Om een verschillende ionen beter in de hybride silica matrix te dispergeren werd in hoofdstuk $\mathbf{7}$ een alternative hybride silica precursor gesynthetiseerd met een gebrugde malonamide groep, namelijk: $N, N_{,} N^{\prime}, N^{\prime}-$ tetrakis(triethoxysilylpropyl)malonamide, wat we voor het gemak afgekort was als TTPMA. Deze entiteit zou ons in staat stellen metal ionen te dispergeren in de hybride silica precursor alvorens de condensatie reactie met behulp van een in ethanol verdunde water/salpeterzuur mengel in 
werking werd gezet. Hierbij werd gekozen voor de incorporatie van cerium en nikkel ionen die in potentie affiniteit zullen vertonen met respectievelijk $\mathrm{CO}_{2}$ en $\mathrm{H}_{2}$ gas. Ondanks het feit faseseparatie niet helmaal vermeden kon worden en gaspermeatiemetingen geen aanwijzingen vertoonden voor een veranderde affiniteit voor $\mathrm{CO}_{2}$ en/of $\mathrm{H}_{2}$ was er zowel in het geval van nikkel als cerium gemixte TTPMA een verbeterde selectiviteit waargenomen ten opzichte van eerder gerapporteerde BTESE-membranen. Een verbetering dat waarschijnlijk geassocieerd moet worden met een betere poriegrootteverdeling. 
Acknowledgement. 
A promotion is a little difficult without the help of others and consequently I should thank some that made it possible. First of all I should thank my promoter André ten Elshof for his guidance. André is a nice supervisor who gave me always much confidence and most of the time he sees the bright perspectives of particular results. I had always appreciated his optimism. Beside that I could always walk into his office whenever I needed advise.

Moreover, I would like to thank Tomek as a supervisor (during my masters), colleague and good friend. We shared much thoughts, particularly, concerning small angle X-ray scattering (SAXS). Together we dove into the theory of this phenomena and we became a bit the SAXS-experts of the group. I also like to thank you and Kasia for nice trips to Gogol Bordello, Leeds and a memorable birthday party of your daughter Maja in Poland.

Besides that, I like to thank all the other members of the SAXS-clan (Sjoerd, Hessel, Suresh, Sajid, Pablo en Huiyu) for the nice trips to the European Synchrotron Radiation Facility (ESRF) in Grenoble, the explorative measurements and interesting discussions. Of course non-stop measurements at the beamline for 3-4 days and a planning that was susceptible to modifications, sometimes gave some tensions and insomnia. Nevertheless, during these trips we really get to know each other and I these experiences were very useful to me. Also, I must thank the technicians of the Dutch Belgium beamline (DUBBLE, Bm26). Particularly I would like to thank Guiseppe, however also Daniel, Fabian and Wim for their preparative work, assistance and their willingness to solve issues after office hours.

Furthermore, I will tank a few people for measurements that were performed outside the inorganic materials science (IMS) group: Therefore, I firstly like to thank Leo and Sabarinathan from the University of Ausburg for the solid state Magic angle spinning nuclear magnetic resonance spectroscopy (solid-state MAS NMR) measurements on mixed niobia hybrid silica systems. Particularly, his expertise of the exotic ${ }^{17} \mathrm{O}$ but also on ${ }^{29} \mathrm{Si}$ and ${ }^{13} \mathrm{C}$ provided a useful insight in the structural connection being formed within these glassy materials (chapter 5 ).

Secondly, I would like to thank Martin Lutz and Vadim Kessler for single crystalline X-ray diffraction measurements on the $\mathrm{Nb}_{8} \mathrm{O}_{10}(\mathrm{OEt})_{20}$ crystallites. Thirdly, I would like to thank Hessel for a constructive cooperation within the STW-project (National Institute of Technical Sciences). Particularly 
concerning the chemical stability measurements, however we also cooperated in the field of SAXS. Although sometimes, he overloaded me with lots of ideas, it mostly contained some useful information. Additionally I should also thank Hammad, Arjan and Louis for a good cooperation with the inorganic membranes group (IM). Particularly, with Hammad I worked together in applying mixed metal oxide hybrid silica for membrane applications. Moreover, I thank Mariëlle, Jaap en Wim for their help and cooperation in coating tubular membranes as well as I appreciated their critical input which kept me thinking further. Also, I would like to thank all the other members of the STW: Rob (ECN), Frans (Pervatech), Monique (STW), Arjan (Shell), Gerald (Akzo Nobel) and Gadi (University of Amsterdam) for their constructive interferences and financial support.

Next, I would like to thank the technicians Henk en Dominic which are crucial to our group and I could reach them for all sorts of technical issues. Dominic helped me substantially with the design and fabrication of a SAXSmixing setup, which eventually led to a nice chapter (chapter 3 ). With Henk I shared much thoughts concerning several issues as a flowbox, peltier reactor cooling systems and a mixing setup. It was handy to gain some good advice from an experienced technician.

Furthermore I would like to thank the lab course students David and Kristianne that I had under my supervision for their orienting work. I let them suffer with complex spectroscopic data, which they manage quite well. I also like to thank Robin a bachelor student who performed some research on the TTPMA precursor. With you it was always a surprise what happens in the lab.

Moreover, I like to thank everyone from IMS, including my roommates Michiel, Janneke, Victor, Huiyu, Robert, Mohammed and of course Wouter. Wouter managed to survive 4 years with me in one office and we shared a lot of thoughts. Also, I liked working in the lab with the chemical brothers including: Maarten, Antony, Pablo, Huiyu, Michiel, Gerard, Tomek, Ronald, Ole, Suresh, Nicolas, Petra, Liz, Joren, Sajid, Sjoerd and Wouter (3x). It was a nice place where we gained lots of new ideas and we always made time for some Friday afternoon experiments. Yet, is also like to thank all the other members of IMS for a great memorable time and nice trips to Smallenberg and Oxford, including: Dave, Mark, Bernard, Guus, Gertjan, José, Josée, Marion, Peter $(2 x)$, Alim, Nirupam, Bouwe, Ruud, Kurt, Brian, Hajo, Michelle, Rik, Tom, Werner, Anirban, Kenan, Deba, Minh, Anuj, Evert, Ben, 
Tjeerd, Zhaoliang, Xin, Hans, Oktay, Gerwin, Tülin, Petra, Vera, Bahruz, Maarten en Roy.

Close to the end of the acknowledgement, I would like to thank a few good friends for a great period. Including, a former house-mate and a true chemist, Michiel Beij. I like the way that we can fantasize and speculate about new possibilities. Also, I would like to thank formal IMS-er Sandra Stadman and I share her passion for nice food and spices. In addition, I would the whole marksman guild St. Jan Keijenborg, where I Played the trumpet, since the age of 11 for the nice trips and events.

Last, but not least, I would like to thank my family for their support: My brother Maurits with Ritagna, my sister Bernadette with Bas and my farther Wim. Of course, I would like to thank my mother Alice, who I lost almost 10 years ago. Dear Alice, you were a great mother and I believe you may be a bit proud on me. 


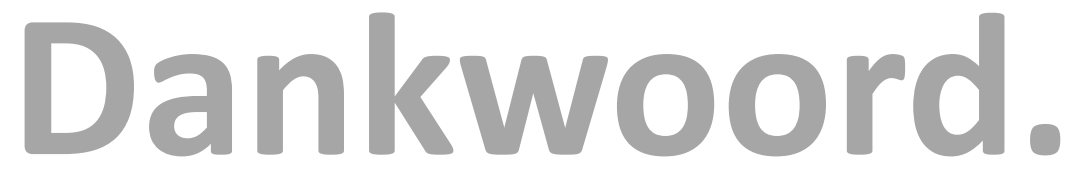


Promoveren gaat natuurlijk niet zonder de hulp van anderen en ik wil dan ook een paar mensen bedanken. Allereerst wil ik mijn begeleider/promotor André bedanken. André is een fijne begeleider, heeft mij altijd veel vertouwen gegeven en bekijkt resultaten vrijwel altijd met een positieve insteek. Zelfs bij moeilijk verklaarbare metingen waar ik niet zoveel heil in zag. Daarnaast kon ik eigenlijk altijd bij zijn kantoor binnen komen waaien als ik advies nodig had. Ik heb veel nuttig advies van hem gekregen met name in het schrijven van artikelen waar ik volgends mij wel aardig in gegroeid ben.

Eveneens wil ik Tomek bedanken als begeleider (gedurende mijn master), collega en goede kameraad. We hebben veel van gedachte gewisseld, met name op het gebied van kleine hoek röntgen verstrooiing (SAXS). Samen zijn we in de theorie van dit verschijnsel gedoken en werden we toch een beetje de SAXS goeroes van onze vakgroep. Ook wil ik jou en Kasia bedanken voor de leuke uitjes naar Gogol Bordello, Leeds en de aparte vakantie met de eerste verjaardag van je dochter Maja in Polen.

Daarnaast wil ik alle andere leden van de SAXS clan (Sjoerd, Hessel, Suresh, Sajid, Pablo en Huiyu) bedanken voor de interessante exploratieve metingen, nuttige discussies en de gezelligheid tijdens de tripjes naar de Europese Synchrotron Straling Faciliteit (ESRF) in Grenoble. Natuurlijk, nonstop meten op de röntgenbundel voor 3-4 dagen en een planning die continue aangepast werd, gaf soms wat strubbelingen, slapeloosheid en stress. Toch was het een erg nuttige ervaring en leer je elkaar zo wel beter kennen. Tevens wil ik de technici van de Nederlands/Belgische röntgenbundel (DUBBLE Bm26) bedanken. Met name Guiseppe, maar ook Daniel, Fabian en Wim voor het voorbereidende en assisterende werk hun probleem oplossend denkvermogen, en de bereidheid om nog even door te werken om problemen opgelost te krijgen.

Ook heb ik een aantal metingen buiten IMS laten doen: Daarvoor wil ik allereerst Leo en Sabarinathan van de universiteit in Ausburg bedanken voor de vaste stof magische spinninghoek kernspinresonantie spectroscopie metingen (solid state MAS-NMR) op gemixte niobia hybride silica systemen. 
Met name zijn expertise op het gebied van de exotische ${ }^{17} \mathrm{O}$ kern alswel op de ${ }^{29} \mathrm{Si}$ en ${ }^{13} \mathrm{C}$ kernen gaven een goed inzicht in de structurele verbindingen die in het glasachtig materiaal gevormd werden (hoofdstuk 5).

Ten tweede zou ik graag Martin Lutz en Vadim Kessler willen bedanken voor de éénkristal röntgendiffractiemetingen op de $\mathrm{Nb}_{8} \mathrm{O}_{10}(\mathrm{OEt})_{20}$ kristallieten (hoofdstuk 6). Ten derde wil ik Hessel bedanken voor de fijne samenwerking binnen het project van STW (Stichting Technische Wetenschappen). Met name met betrekking op de chemische stabiliteit metingen, maar we hebben eveneens samengewerkt op het gebied van SAXS. Ik waardeer zijn eigenzinnige karaker en hoewel hij mij soms overviel met een spaakwaterval aan woorden rollen er meestal wel een aantal nuttige tips uit. Natuurlijk gaat mijn dank ook uit naar Hammad, Louis en Arjan binnen de anorganische membranen group (IM). Met Hammad heb ik samengewerkt om mijn ideeën met betrekking tot metaal oxide gedoopte hybride silicaten toe te passen in membranen. Met de tetrakis(triethoxysilylpropyl)-malonamide (TTPMA) systemen heeft het uiteindelijk wel tot aardige resultaten geleid (Hoofdstuk 7). Daarnaast wil ik Mariëlle, Jaap en Wim van het Nederlandse energie onderzoekscentrum (ECN) bedanken voor de samenwerking met het coaten van cilindrische membranen. Tevens waardeer ik hun kritische input, dat me weer verder aan het denken heeft gezet. Ook wil ik alle andere leden van STW: Rob (ECN), Frans (Pervatech), Monique (STW), Arjan (Shell) Gerald (Akzo Nobel) en Gadi (Universiteit van Amsterdam) voor het constructief meedenken en de financiële ondersteuning van mijn project.

Daarnaast zou ik de technici van onze vakgroep Henk en Dominic bedanken. Goede technici zijn cruciaal, en ik kon vrijwel altijd bij ze terecht voor allerlei technische probleempjes. Dominic heeft me ontzettend veel geholpen met het ontwerp en de bouw van een mix-setup voor SAXS wat me uiteindelijk toch weer een mooi hoofdstuk heeft opgeleverd (hoofdstuk 3). Samen met Henk heb ik onder andere gebrainstormd voor een extra stikstofdoorstroombox (flowbox) voor reacties die gevoelig zijn voor water, aangezien er in de huidige handshoenenbox (glovebox) weinig ruimte over was voor chemische reacties. Het is een heel degelijke box geworden. Het 
kwam voor mij helaas een beetje als mosterd na de maaltijd, maar het is sowieso nuttig voor nieuwe promovendi zoals Petra. Ik wens haar er dan ook veel plezier mee.

Tevens wil ik de practica-studenten David en Kristianne bedanken. In heb hen veelal opgezadeld met lastig interpreteerbare infrarood en kernspinresonantie spectroscopie data waar zij zich, met enige begeleiding, toch karig doorheen geworsteld hebben. Ook wil ik Robin voor zijn oriënterend onderzoek naar de TTPMA precursor. Het was altijd feest met jouw in het lab en je hebt dan ook een onvergetelijke indruk gemaakt.

Eveneens wil ik de IMS-ers bedanken voor een fijne PhD-periode. Waaronder mijn kantoorgenoten: Michiel, Janneke, Victor, Huiyu, Victor, Robbert, Mohammed en in het bijzonder Wouter. Wouter, met wie ik 4 jaar op het kantoor heb gezeten, was meestal mijn eerste aanspraakpunt met allerlei gedachtekronkels en lichte frustraties. Ook buiten IMS zijn we veel opgetrokken, zo nu en dan schaatsen waar ik toch al wel een stuk beter in geworden ben. Daarnaast wil ik alle chemische broeders bedanken: Maarten, Antony, Pablo, Gerard, Michiel, Tomek, Sjoerd, Ole, Suresh Ronald, Huiyu, Sajid, Nicolas, Ronald, Wouter (3x), Joren, Liz en Petra. Het chemisch lab was een relaxte werkplek waar tussen de experimenten veel idiote ideeën gespuid werden en vaak wel tijd gemaakt werd voor een vrijdagmiddag experimentje.

Natuurlijk wil ik ook alle andere IMS-ers bedanken die ik nog niet genoemd heb: Dave, Mark, Bernard, Guus, Gertjan, José, Josée, Marion, Peter (2x), Alim, Nirupam, Bouwe, Ruud, Kurt, Brian, Hajo, Michelle, Rik, Tom, Werner, Anirban, Kenan, Deba, Minh, Anuj, Evert, Ben, Tjeerd, Zhaoliang, Xin, Hans, Oktay, Gerwin, Tülin, Petra, Vera, Bahruz, Maarten en Roy. Ik wil hen bedanken voor een onvergetelijke tijd en gezellige uitjes naar Smallenberg en Oxford.

Tegen het einde van deze ietwat lange dankwoord wil ik nog een paar goede vrienden bedanken voor een mooie periode. Waaronder Michiel Beij een oud huisgenoot uit Deventer en een chemicus pur sang. Ik vind het mooi over hoe wij over van alles kunnen speculeren en fantaseren. 
Daarnaast wil ik ex-IMS-ster Sandra Stadman ook nog even noemen. Ik deel haar liefde voor lekker eten, maar ze kan veel beter taarten bakken als ik. Ook wil ik het hele schuttergilde St. Jan Keijenborg, waar ik al sinds mijn 11e trompet bespeel, voor de nodige ontspanning, de gezellige uitjes met concoursen, oktoberfest en dergelijke.

Tenslotte, wil ik mijn familie bedanken voor alle steun: Mijn broer Maurits met Ritagna, mijn zus Bernadette met Bas en mijn pa Wim. Natuurlijk wil ik ook mijn moeder bedanken wie helaas al bijna 10 jaar heb moeten missen. Beste, Alice je was een geweldige moeder en ik denk ook wel dat nu wel een beetje trots op mij zult zijn. 